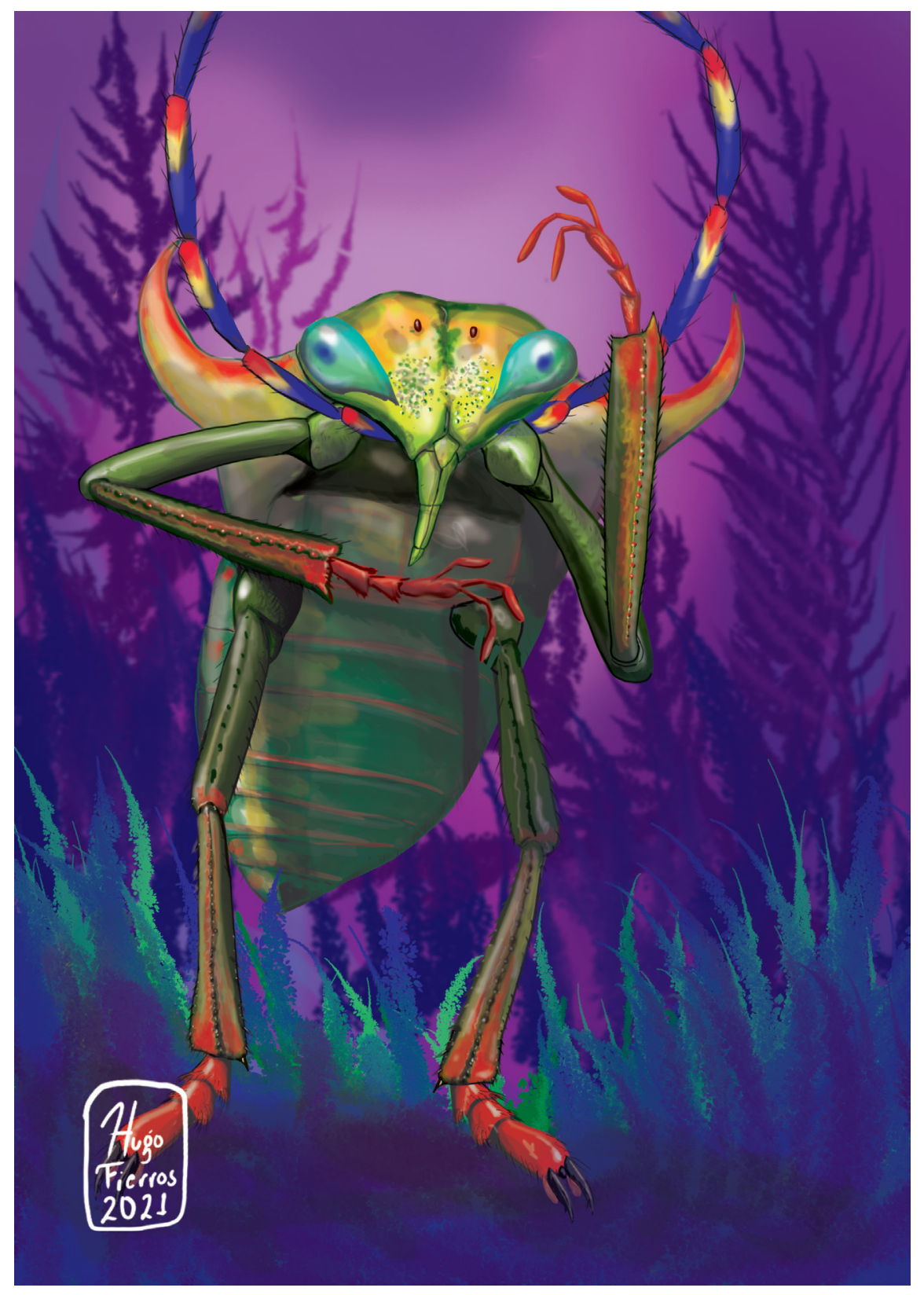

Dugesiana, Año 29, No. 1, (enero-junio, primer semestre 2022), es una publicación semestral, editada por la Universidad de Guadalajara, a través del Centro de Estudios en Zoología, por el Centro Universitario de Ciencias Biológicas y Agropecuarias. Camino Ramón Padilla Sánchez \# 2100, Nextipac, Zapopan, Jalisco, Tel. 37771150 ext. 33218, http://148.202.248.171/dugesiana/index.php/DUG/index, glenusmx@gmail.com. Editor responsable: José Luis Navarrete-Heredia. Reserva de Derechos al Uso Exclusivo 04-2009-062310115100-203, ISSN: 20079133, otorgados por el Instituto Nacional del Derecho de Autor. Responsable de la última actualización de este número: José Luis Navarrete-Heredia, Editor y Ana Laura González-Hernández, Asistente Editorial. Fecha de la última modificación 1 de enero de 2022, con un tiraje de un ejemplar.

Las opiniones expresadas por los autores no necesariamente reflejan la postura del editor de la publicación.

Queda estrictamente prohibida la reproducción total o parcial de los contenidos e imágenes de la publicación sin previa autorización de la Universidad de Guadalajara. 
An annotated checklist of ground beetles (Insecta: Coleoptera: Carabidae) occurring in the state of Sonora, Mexico with descriptions of new species and notes about some collection localities

\author{
Lista anotada de carabidos (Insecta: Coleoptera: Carabidae) que ocurren en el estado de Sonora, Méxi- \\ co, con descripción de nuevas especies y notas sobre algunas localidades de colecta
}

\author{
"Danny Shpeley and **Thomas R. Van Devender \\ * Department of Biological Sciences, University of Alberta, Edmonton, Alberta, T6G 2E9, Canada; \\ email: dannyshpeley@gmail.com; ${ }^{* *}$ Greater Good Charities, 6262 N. Swan Road, Suite 165, Tucson, \\ Arizona 85718, USA; email: yecora4@comcast.net
}

\begin{abstract}
An annotated checklist of ground beetles in the family Carabidae from the state of Sonora in Mexico is presented with notes including number of specimens examined, municipios, habitat, vegetation, elevation, and collection dates. The checklist includes 388 taxa, of which 270 are described taxa (included are 19 subspecies and 1 hybrid) and 118 are unnamed morphospecies, in 92 genera representing 35 tribes. Dot maps display where specimens were collected. Three new species are described, with corrected keys and images: Calathus mcclevei, new species (type locality: Mexico, Sonora, Municipality of Yécora, $10 \mathrm{~km}$ south of Yécora, ca. $28.278^{\circ} \mathrm{N} 108.926^{\circ}$ W); Calathus tigrinus, new species (type locality: Mexico, Sonora, Municipality of Nacozari de García, Rancho El Tigre, $28.3 \mathrm{~km}$ (by

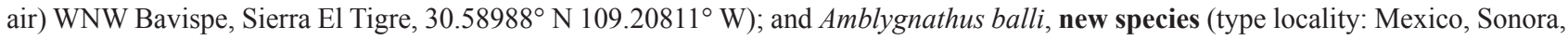
Municipality of Fronteras, Rancho Capulín, $23.5 \mathrm{~km}$ (by air) WSW Fronteras, Sierra Buenos Aires, 30.76167 N 109.82361 ${ }^{\circ} \mathrm{W}$ ).
\end{abstract}

Key Words: Biogeography, checklist, Carabidae, habitats, new species, Sonora, vegetation.

\title{
RESUMEN
}

Se presenta una lista comentada de los escarabajos de la familia Carabidae del estado de Sonora, México, incluye número de ejemplares examinados, municipios, hábitat, vegetación, elevación, y fechas de colecta. El listado contiene 388 taxones, de los cuales 270 son taxones descritos (se incluyen 19 subespecies y un híbrido) y 118 son morfoespecies sin nombre, en 92 géneros de 35 tribus. Con mapas de puntos se muestran los lugares de colecta. Se describen tres especies nuevas, con claves corregidas e imágenes: Calathus mcclevei, especie nueva (localidad tipo: México, Sonora, Municipio de Yécora, $10 \mathrm{~km}$ al sur de Yécora, ca. $28.278^{\circ} \mathrm{N} 108.926^{\circ} \mathrm{W}$ ); Calathus tigrinus, especie nueva, (localidad tipo: México, Sonora, Municipio de Nacozari de García, Rancho El Tigre, $28.3 \mathrm{~km}$ (por aire) WNW Bavispe, Sierra El Tigre, $30.58988^{\circ} \mathrm{N} 109.20811^{\circ} \mathrm{W}$ ); y Amblygnathus balli, especie nueva (localidad tipo: México, Sonora, Municipio de Fronteras, Rancho Capulín, 23.5 km (por aire) WSW Fronteras, Sierra Buenos Aires, $30.76167^{\circ} \mathrm{N} 109.82361^{\circ} \mathrm{W}$ ).

Palabras Clave: Biogeografia, listado, Carabidae, hábitats, especies nuevas, Sonora, vegetación.

This is the first regional checklist of carabid beetles collected in the state of Sonora in Mexico. The authors would like to note that this list of species is not complete, and no attempt was made to assemble all of the carabid specimens collected in Sonora, nor did they personally sample many parts of Sonora, and as such, this checklist is a starting point for someone wishing to do further studies on Sonoran carabids. Approximately one third of the species in our checklist are not named, which reflects the paucity of current literature dealing with the taxonomy of the family Carabidae in Mexico.

\section{BIOGEOGRAPHY AND VEGETATION}

Sonora is a large state in northwestern Mexico. It is bound on the west by the Gulf of California and to the east by the Sierra Madre Occidental (SMO). To the north is the border with Arizona and a broad transition into the American Southwest. Although the Tropic of Cancer is at $23.4^{\circ} \mathrm{N}$, just north of Mazatlán, Sinaloa, is often considered the northern limits of the New World tropics, the transition into the northern temperate zone is at about $29^{\circ} \mathrm{N}$ in east-central Sonora, $680 \mathrm{~km}$ to the north-northwest. The northernmost tropical deciduous forest (TDF) is in the Sierra San Javier $\left(28.6^{\circ} \mathrm{N} ; 300 \mathrm{~km}\right.$ south of the Arizona border; Martin et al. 1998). Thornscrub is the transitional vegetation between TDF and adjacent biotic communities. Coastal thornscrub (CTS) is on the coastal plain of the Gulf of California as far north as Guaymas $\left(28.0^{\circ} \mathrm{N}, 110.8^{\circ} \mathrm{W}\right)$, where it transitions into Sonoran desertscrub. Foothills thornscrub (FTS) is inland on rocky slopes as far north in river valleys to ca. 104 $\mathrm{km}$ south of the Arizona border ( $30.4^{\circ} \mathrm{N}$; Van Devender et al. 2013a). FTS is transitional with Sonoran desertscrub to the west; oak woodland in the foothills of the Sierra Madre Occidental (SMO) to the east and on Sky Island mountain ranges in northeastern Sonora; and desert grassland in the north (Van Devender et al. 2013a).

At higher elevations and latitudes with colder winter temperatures, more temperate vegetation is present. On 
the Chihuahua-Sonora border to the east, the SMO reaches its northern limit in Sonora in the Sierra Huachinera (ca. $30.3^{\circ} \mathrm{N}$ ). The vegetation zonation in the Yécora area in the SMO in easternmost Sonora is FTS, TDF, oak woodland, and pine-oak forest (Van Devender and Reina-G. 2016). The wettest, most diverse floristic area in the SMO is from Yécora to Mesa Tres Ríos in the Madrean Tropical Floristic Subdivision in eastern Sonora (González-E. et al. 2012).

In the Madrean Archipelago (= the Sky Islands Region) between the SMO and the Mogollon Rim in central Arizona, there are 55 isolated Sky Island mountain ranges or complexes of ranges connected by oak woodland corridors; 32 of them are in Sonora (Deyo et al. 2013, Van Devender et al. 2013b). Sky Islands crowned with oak woodland or pine-oak forest emerge from lowland "seas" of Sonoran and Chihuahuan desertscrub, desert grassland, FTS or TDF. The Madrean Archipelago is a convergence zone for species from the cold temperate Rocky Mountains and Colorado Plateaus; Mediterranean/Californian chaparral below the Mogollon Rim in Central Arizona; the Great Plains and Chihuahuan Desert from the east; the Sonoran Desert to the west; and the New World tropics to the south.

The Sonoran Desert covers the western half of Sonora (Turner 1994). The Plains of Sonora Subdivision dominated by mixed legumes and organ pipe cactus (Stenocereus thurberi (Engelm.) F. Buxb.) is present in much of central Sonora. The Arizona Upland Subdivision in north-central Sonora is dominated by saguaro (Carnegiea gigantea (Engelm. (Britton \& Rose)) and foothills paloverde (Parkinsonia microphylla Torr.). The Central Gulf Coast Subdivision along the Gulf of California in both Sonora and Baja California is dominated by diverse cacti and succulent shrubs. Near the coast there are saltscrub on saline flats and dunescrub on sand dunes. The Lower Colorado River Valley Subdivision in northwestern Sonora is hyperarid with stark desert mountain ranges, a massive inland sand sea, and sandy flats with sparse desertscrub (Turner 1994).

Riparian vegetation is not part of the normal vegetation gradient but dissects the landscape in a linear fashion. Riparian habitats are not restricted to wet rivers or streams but are ecological features of drainages at all elevations. They are more mesic than adjacent vegetation types, harvesting water, nutrients, and seeds from large areas. In southern Sonora, tropical riparian communities line arroyos and rivers with guásima (Guazuma ulmifolia Lam.) and Mexican bald cypress/sabino (Taxodium distichum (L.) Rich. var. mexicanum (Carrière) Gordon). In northeastern Sonora, the Fremont cottonwood (Populus fremontii S. Watson)-willow (Salix spp.) riparian deciduous forest typical of Arizona is present. Xeroriparian vegetation mostly dominated by velvet mesquite/mezquite (Prosopis velutina Wooton) is along arroyos throughout drier parts of Sonora (Van Devender et al. 2010).

\section{HABITATS}

Carabids were collected in most of the major habitats in Sonora, including TDF (Figure 1B), and CTS and FTS (Figure 2). Temperate habitats sampled include desert grassland (Figure 3), oak woodland (Figure 4), and pineoak forest (Figure 5). Carabids were also collected in arid desertscrub in the Central Gulf Coast (Figure 6A), Plains of Sonora (Figure 6B), Arizona Upland (Figure 7A), and the Lower Colorado River Valley (Figure 7B) subdivisions of the Sonoran Desert. Riparian habitats are linear vegetation along rivers, streams, dry arroyos, etc. (Figure 8) that dissect other vegetation types at all elevations along the vegetational gradient. These habitats often support carabids as they harvest moisture and nutrients from drier upland habitats. Highways provide access to the various vegetation types they pass through. The best sampled habitats are places like Álamos surrounded by natural vegetation that are often visited cultural sites (Figure 1A). Oak woodland in the Sonoran Sky Island mountain ranges has been relatively well sampled because of the Madrean Archipelago Biodiversity (MABA) program at Sky Island Alliance and the Madrean Discovery Expeditions (MDE) program at Greater Good Charities. But other areas with limited access such as the Gran Desierto in the Lower Colorado River Valley, coastal thornscrub, halophytic communities, and mangrove stands along the coast of the Gulf of California, desert grassland, and Chihuahuan desertscrub near the Arizona border in northeastern Sonora have only been casually sampled and surely would yield interesting records.

\section{MATERIALS AND METHODS}

The following records in the checklist are based on 13,646 carabid specimens, the majority of which are housed in the E. H. Strickland Entomological Museum (7,213 specimens), University of Alberta, Edmonton, Alberta, Canada and the California Academy of Sciences (4,220 specimens), San Francisco, California, United States. The remaining 2,213 carabid specimens seen are housed in the following 12 collections: Canadian National Collection of Insects, Ottawa, Ontario, Canada; American Museum of Natural History, New York, New York, United States; Hasbrouck Insect Collection, Arizona State University, Tempe, Arizona, United States; Carnegie Museum of Natural History, Pittsburgh, Pennsylvania, United States; Essig Museum, University of California Berkeley, Berkeley, California, United States; Milwaukee Public Museum, Milwaukee, Wisconsin, United States; Texas A \& M University, College Station, Texas, United States; University of Arkansas Department of Entomology, Fayetteville, Arkansas, United States; University of Arizona Insect Collection, Tucson, Arizona, United States; University of California Riverside Collection, Riverside, California, United States; Universidad Nacional Autónoma de México, Mexico City, Mexico; and Department of Entomology, United States National Museum of Natural History, Smithsonian Institution, Washington, D.C., United States. Only a few records were 

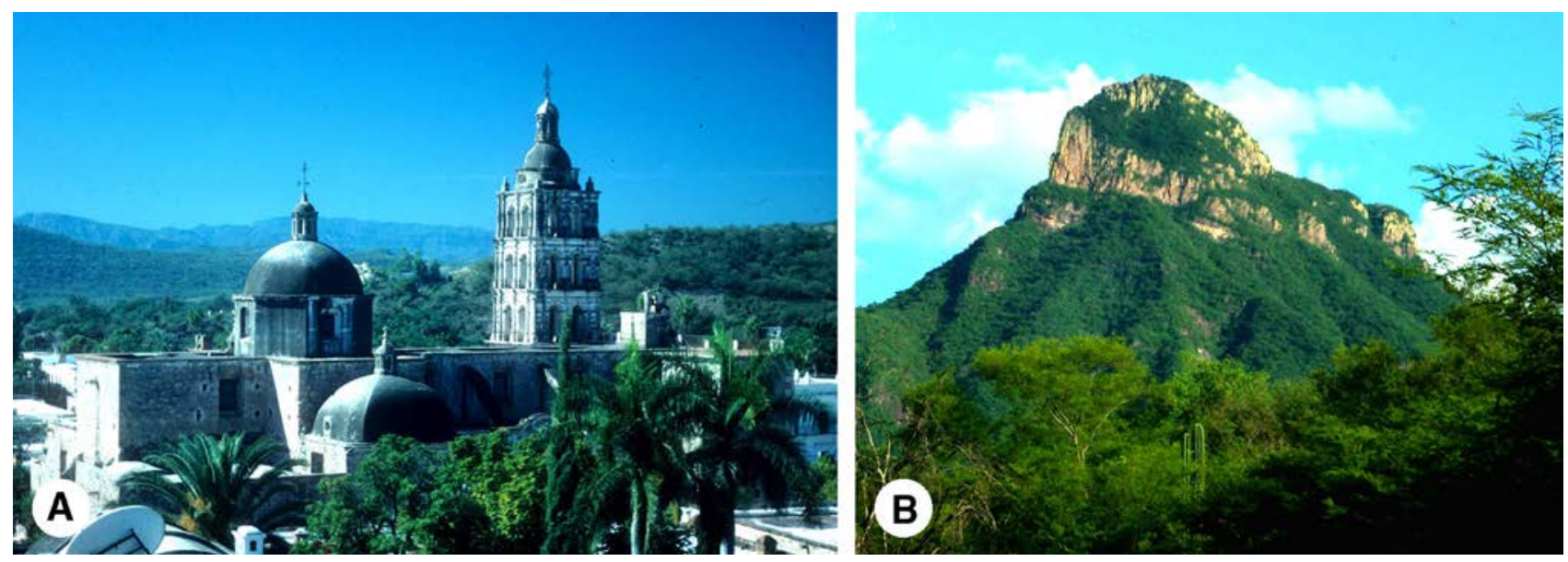

Figure 1. A. Álamos, Sonora. Photo by Van Devender. B. Tropical deciduous forest. Sierra de Álamos. Photo by James C. Rorabaugh.
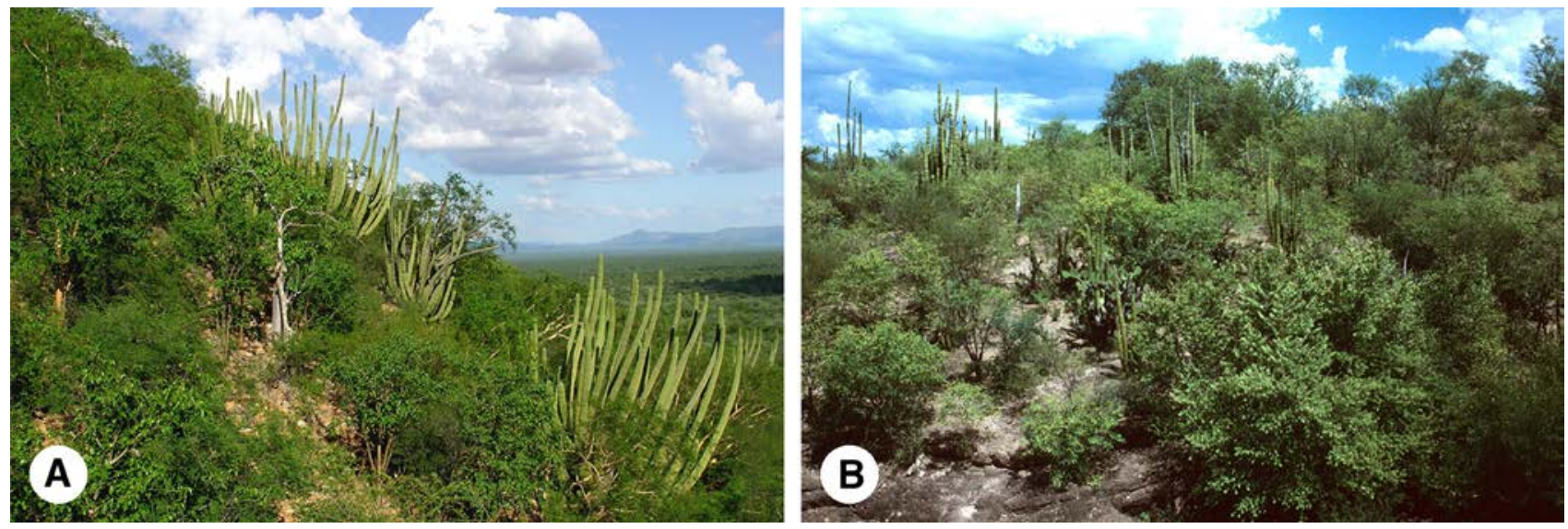

Figure 2. Foothills thornscrub. Visible plants are organpipe cactus/pitahaya (Stenocereus thurberi (Engelm.) F. Buxb.), tree morning glory/palo santo (Ipomoea arborescens (Humb. \& Bonp.) G. Don), papelillo (Jatropha cordata (Ortega) Müll. Arg.), and Durango pricklypear/nopal (Opuntia cf. durangensis Britton \&Rose). A. Photo by Philip C. Rosen. B. Curea. Photo by Van Devender.
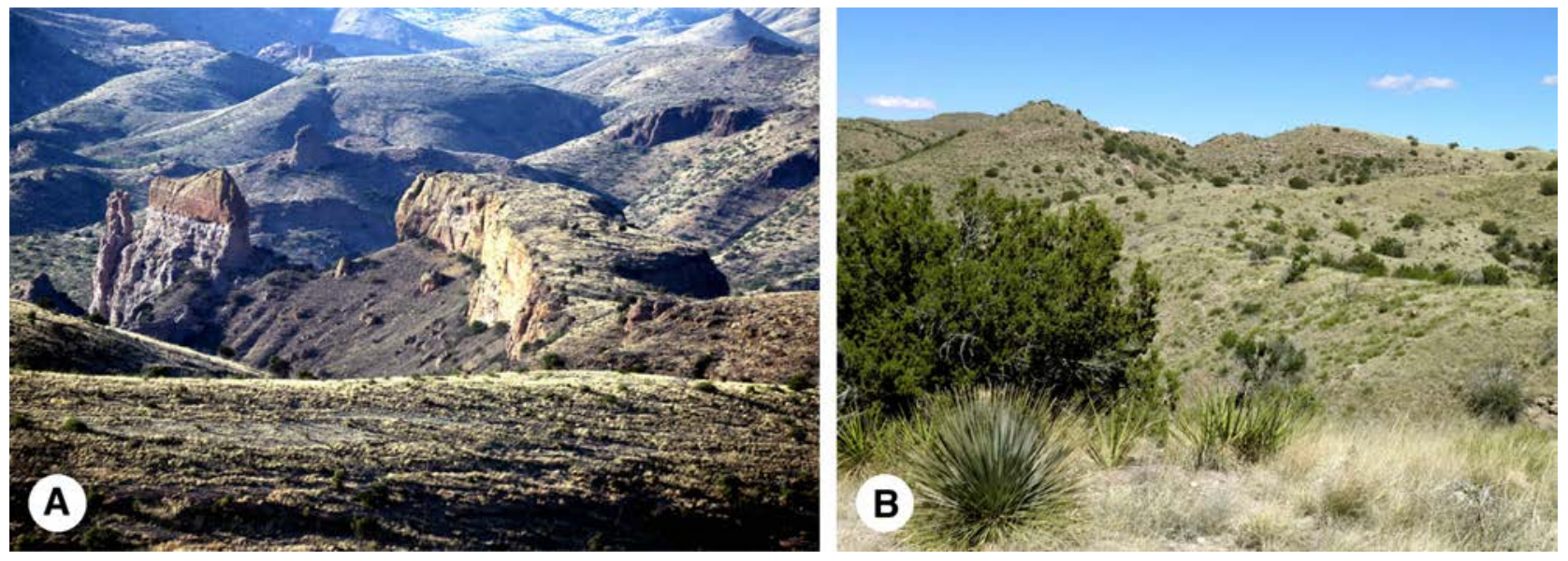

Figure 3. Desert grassland, Sierra San Luis. A. Photo by J. Saucedo. B. Arizonia juniper (Juniperus arizonica (R.P. Adams) R.P. Adams), desert spoon/sotol (Dasylirion wheeleri S. Watson ex Rothr.), and banana yucca/dátil (Yucca baccata Torr.) are visible. Photo by Van Devender. 

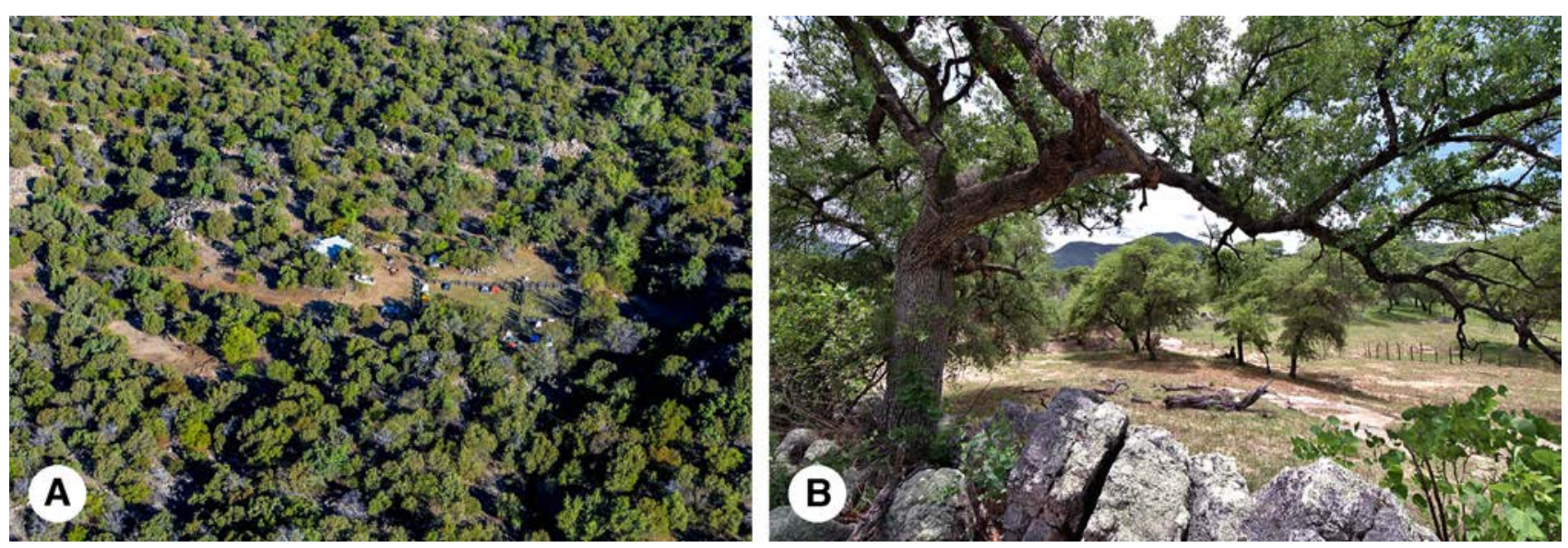

Figure 4. Oak woodland. A. Sierra Chivato. Dominants are Arizona oak/encino blanco (Quercus arizonica Sarg.), Emory oak/bellota (Q. emoryi Torr.), and alligator bark juniper/huata (Juniperus deppeana Steud.). Photo by Luis Gutiérrez, NortePhoto.com. B. Sierra Los Locos. Mexican blue oak/encino azul (Q. oblongifolia Torr.) and coralbean/chilicote (Erythrina flabelliformis Kearney) are visible. Photo by James C. Rorabaugh.
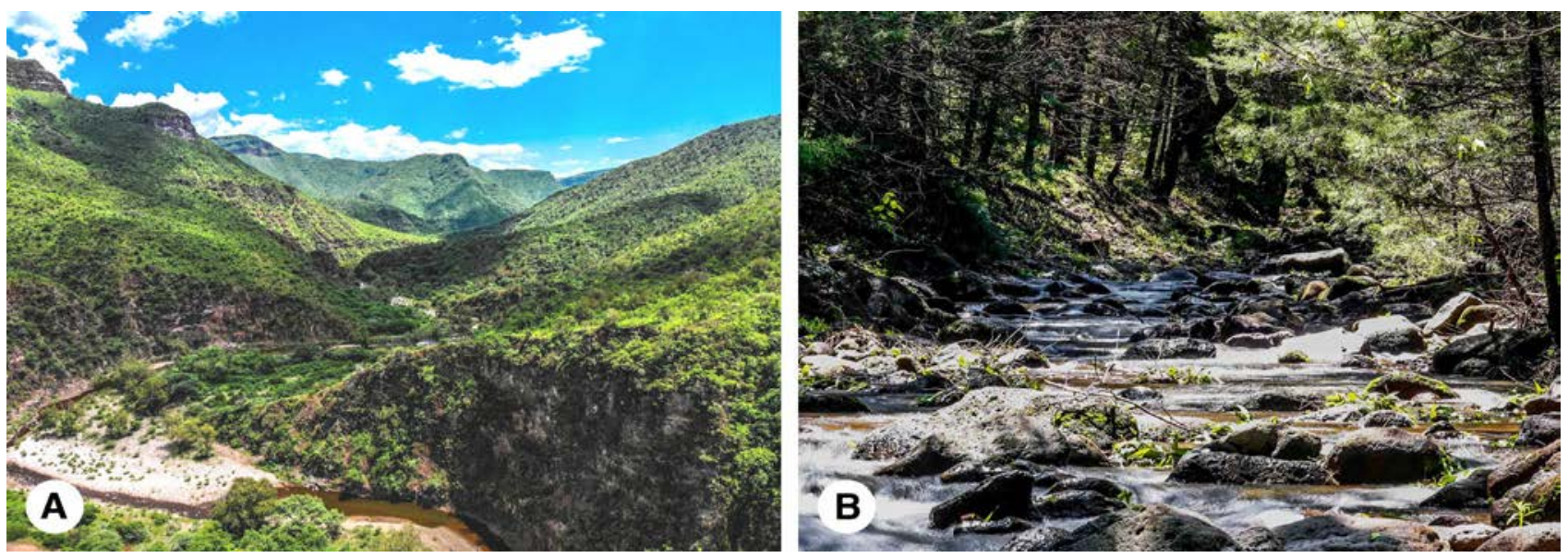

Figure 5. A. Pine-oak forest, aerial view. Mesa Tres Ríos, Sierra Madre Occidental. B. Arroyo La Cueva. Arizona cypress/ sabino (Hesperocyparis arizonica (Greene) Bartel) is the dominant tree. Photos by Luis Gutiérrez, NortePhoto.com.
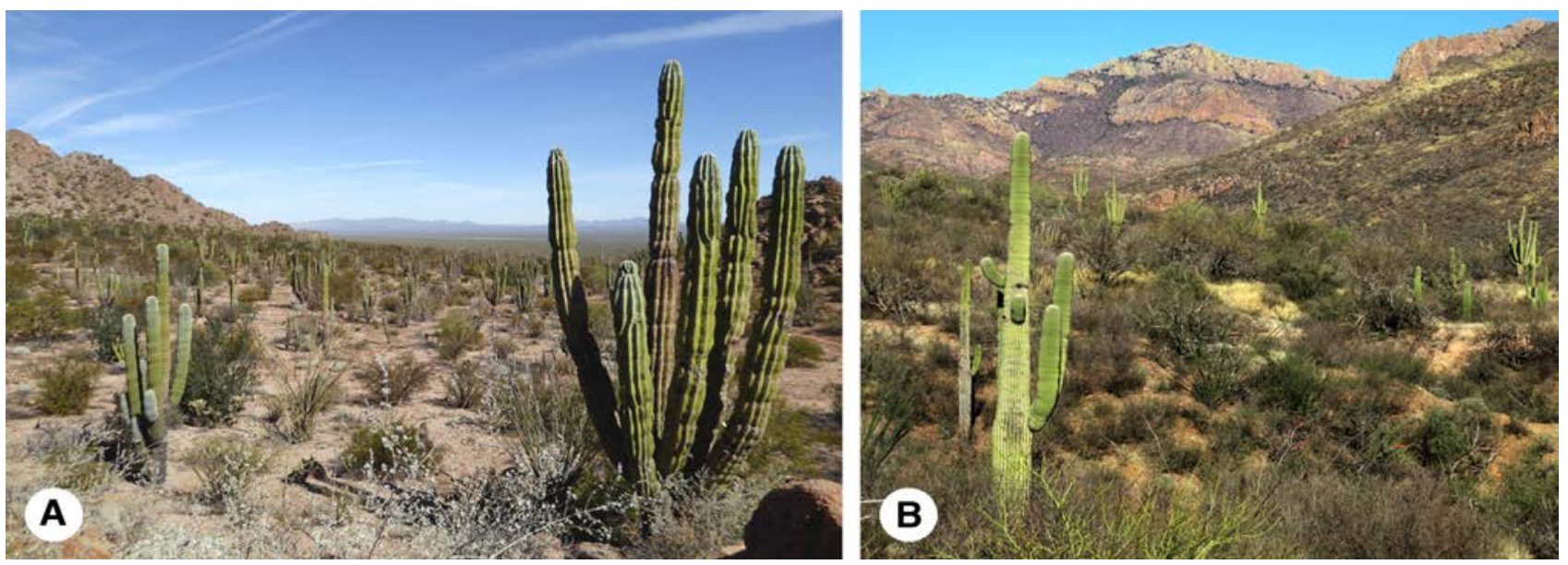

Figure 6. A. Central Gulf Coast desertscrub. Near Puerto Libertad. Dominant columnar cactus is cardón (Pachycereus pringlei (S. Watson) Britton \& Rose). Photo by Ana Lilia Reina-G. B. Plains of Sonora desertscrub, foothills of Sierra Cucurpe. Dominant plants are saguaro (Carnegiea gigantea) and foothill paloverde (Parkinsonia microphylla Torr.). Photo by Doug Danforth. 

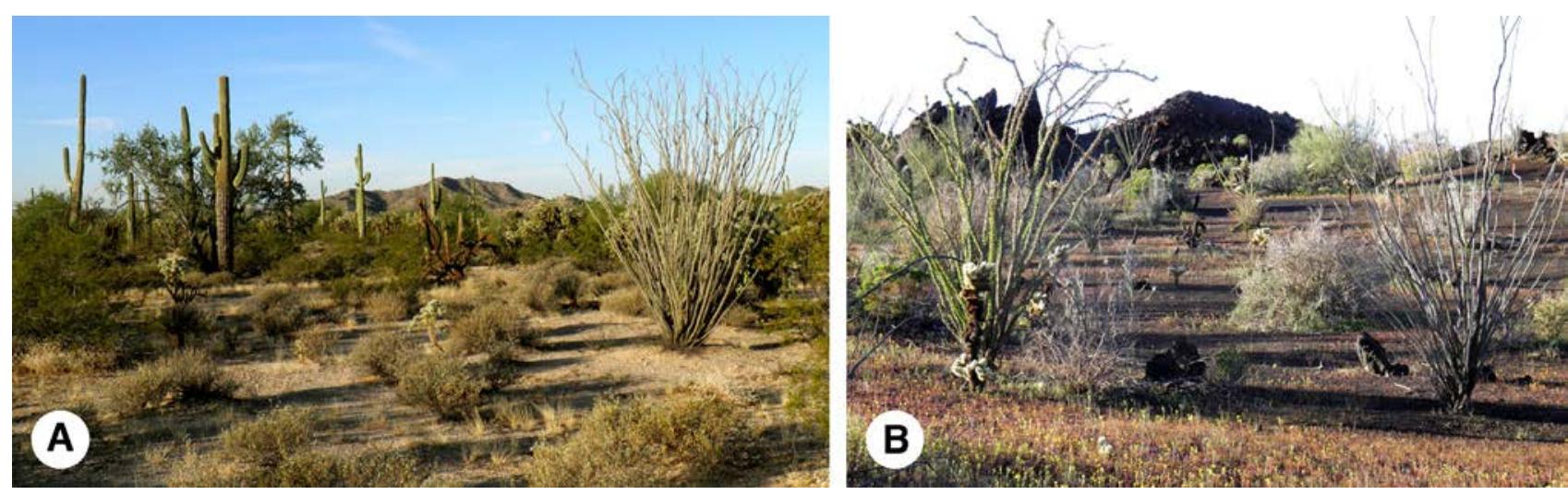

Figure 7. A. Arizona Upland desertscrub. Near Sonoyta. Dominant plants are saguaro (Carnegiea gigantea), desert ironwood/palo fierro (Olneya tesota A. Gray), ocotillo (Fouquieria splendens Engelm.), chainfruit cholla/choya (Cylindopuntia fulgida (Engelm.) F.M. Knuth), and triangleleaf bursage/chicurilla (Ambrosia deltoidea (Torr.) W.W. Payne). B. Lower Colorado River desertscrub. Sierra Pinacate. Dominant plants are ocotillo, white bursage/hierba del burro (A. dumosa (A. Gray) W.W. Payne), and teddybear cholla/choya (C. bigelovii (Engelm.) F.M. Knuth). Photos by Van Devender
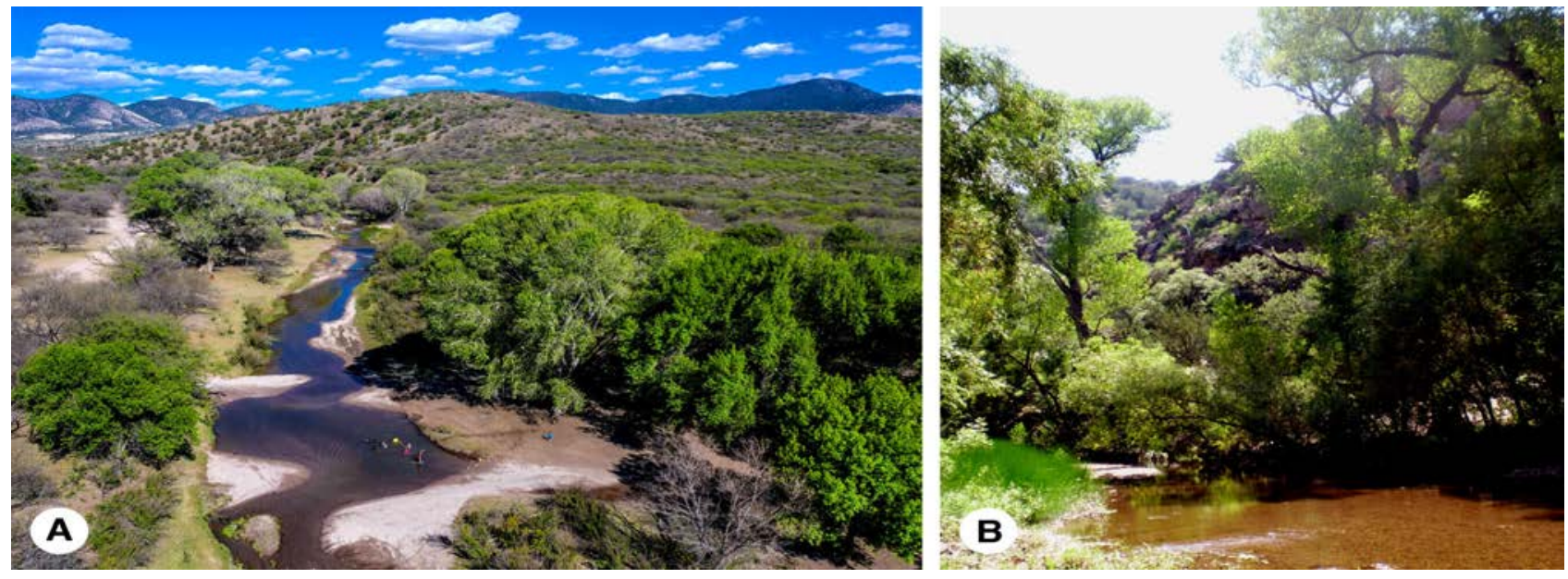

Figure 8. Cottonwood-willow deciduous forests in riparian habitats. Dominants are Fremont cottonwood/álamo (Populus fremontii S. Watson) and Goodding's willow (Salix gooddingii C.R. Ball). A. Santa Cruz River aerial. Photo by Luis Gutiérrez, NortePhoto.com. B. Cajón Bonito Rancho Los Ojos Calientes. Photo by Van Devender.

taken from published literature (Cazier 1954; Gidaspow 1959) and are noted in the checklist. Records for most of the Sonoran carabids cited in this study are publicly available in the MDE database (madreandiscovery.org).

The holotype (male) and one paratype (female) of each of Calathus mcclevei, new species, and Calathus tigrinus, new species, will be deposited in UNAM. Two paratypes (one male, one female) of each of Calathus mcclevei, new species, and Calathus tigrinus, new species, will be deposited in CASC, UAIC, and USNM.

The holotype (male) of Amblygnathus balli, new species, will be deposited in UNAM; the paratype (male) of $A$. balli, new species, will be deposited in PWMC. All of the remaining type material will be housed in the UASM.

CASC Departament of Entomology, California Academy of Sciences, Golden Gate Park, San Francisco, California 94118 , U.S.A.
PWMC Peter W. Messer Collection, Wisconsin, U.S.A.

UAIC University of Arizona Insect Collection, Departament of Entomology, University of Arizona, Tucson, Arizona 85721-0036, U.S.A.

UASM Strickland Museum, Departament of Biological Sciences, University of Alberta, Edmonton, Alberta T6G 2E9 Canada

UNAM Universidad Nacional Autónoma de México, Apartado Postal 70133, 04510, México City, Department Federal, Mexico

USNM United States National Museum of Natural History, Department of Entomology, Smithsonian Institution, Washington, D.C. 20560. U.S.A.

This paragraph applies only to the three new species that are described in this paper-Calathus mcclevei, new species, Calathus tigrinus, new species, and Amblygnathus balli, new species. Total length (TL) in the descriptions 
is the sum of the following three measurements: length of head (HL), measured on the left side from base of mandible to posterior margin of compound eye; length of pronotum (PL) along the midline; and length of longer elytron (EL) from transverse basal line to apex. For the following measurements taken of male genitalia, see Figure 10: length of apical portion (LAP) and width of apical disc (WD). All of the above measurements are named the same as those used by Ball and Nègre (1972). The only other measurement used in the descriptions is length of male median lobe (LML), see Figure 10.

\section{CARABID BEETLE COLLECTING IN SONORA, MEXICO}

Some of the carabids collected in Sonora would have been picked up by entomologists and other researchers who stopped for fuel at service stations while driving through the state. Many carabid species have membranous flight wings and are attracted to the bright lights at the service stations at night. Some appear to be living in cracks around the pumps and buildings. However, most carabids are collected in the field, where different habitats are sampled using different techniques - rolling stones and logs, sift- ing leaf litter, pitfall traps, rotten meat traps, other types of bait traps, beating vegetation, splashing water on pond or stream margins, black lights, mercury vapor lights, and head-lamping at night to name a few. Unfortunately, many of the specimen locality labels do not include how the specimen was collected.

In 1935, Howard S. Gentry collected many carabids in the Rio Mayo region near San Bernardo while collecting plants and fossils. His book Rio Mayo Plants (Gentry, 1942) was published in 1942. In 1953, Borys Malkin collected carabids while in the areas of Hermosillo and Desemboque west-central Sonora and to a lesser extent near Navojoa in southern Sonora. In 1960 and 1963, Paul H. Arnaud, Jr., Edward S. Ross, and David C. Rentz (California Academy of Sciences) collected carabids in the areas of Álamos and Navojoa in southern Sonora, and in the areas of San Carlos Bay and north of Hermosillo in the Sonoran Desert. In 1963 and 1964, Gary R. Noonan collected carabids in the areas of Álamos and Navojoa. Mont A. Cazier, Ronald L. Huber, David L. Pearson, and Norman L. Rumpp collected many tiger beetles in coastal and montane Sonora. Scott McCleve made numerous trips into Sonora from 1980 to 1993 to collect scarabs in the genus Diplotaxis, and generously picked

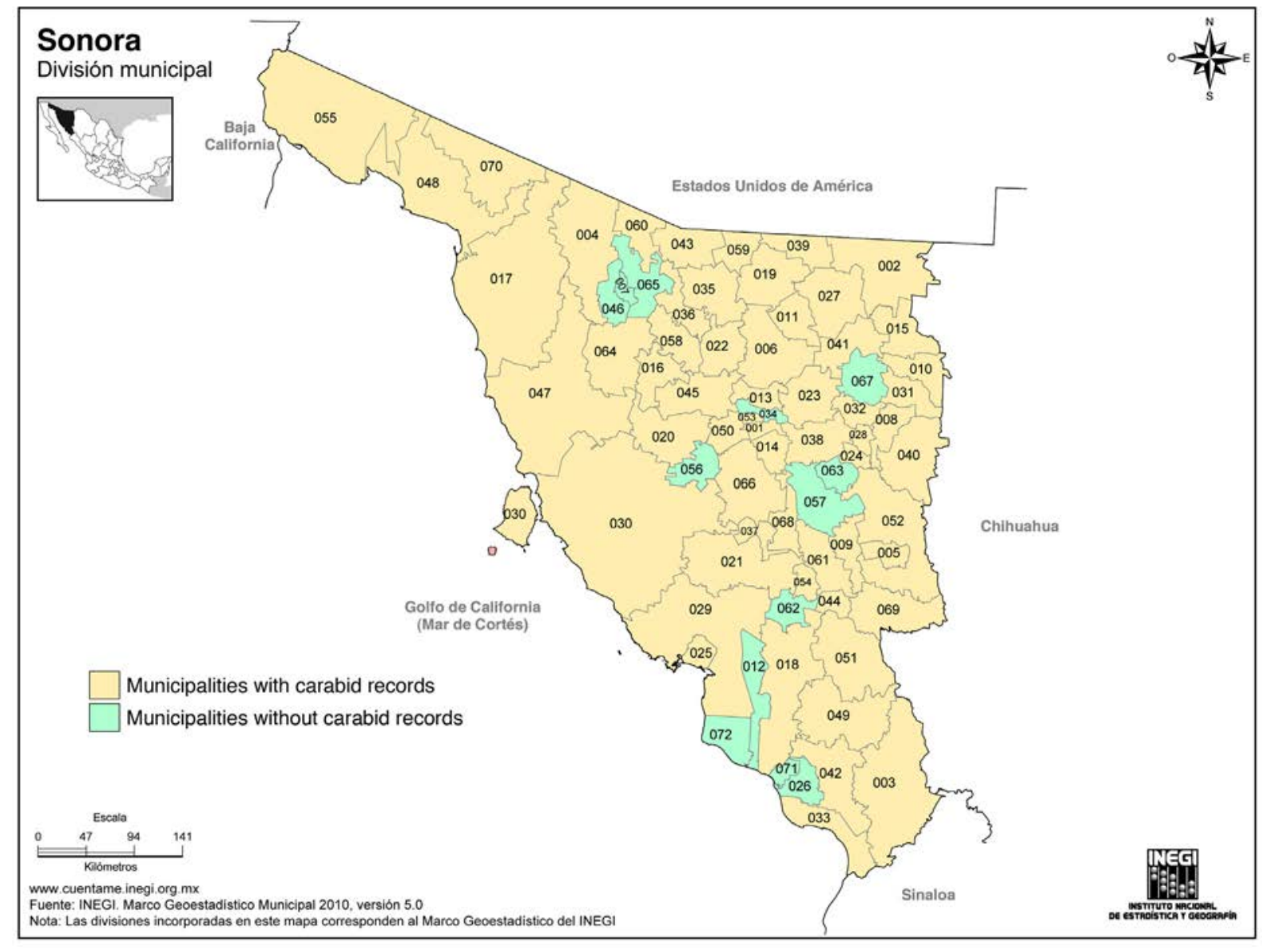

Figure 9. Outline map of the state of Sonora, illustrating the political boundaries of municipalities and those within which carabids have been recorded.

Source of map: http://www.cuentame.inegi.org.mx/mapas/pdf/entidades/div_municipal/sonorampios.pdf 
up carabids and other families of beetles at the same time. Some of Scott's field companions were Annie McCleve (Scott's wife), Peter Jump, Steven J. Prchal, George E. Ball, Kathleen E. Ball, and David R. Maddison.

Six collecting trips, originating from the University of Alberta in Edmonton, Alberta, Canada, passed though Sonora. In 1965, George E. Ball and Donald R. Whitehead collected specimens along MEX 15, near Nogales, Cíbuta, La Atascosa, El Oasis, and Vícam before continuing south into the state of Sinaloa. In 1967, George Ball, Terry L. Erwin, and Robin E. Leech collected specimens along MEX 15, near La Atascosa, Santa Ana, and Hermosillo before continuing to Sinaloa. In 1982, Ball, Kathleen E. Ball, Maddison, and McCleve collected in the Sierra San Luis (Varela Ranch [= Rancho el Valle]) and Huachinera (Rancho el Madroño). They also made collections near San Nicolás (SW of Colonia el Tajo), Nácori Chico, and in the vicinity of Moctezuma, as well as along MEX 15 near Ímuris and Santa Ana. In 1983, Henry E. Frania, Rein J. Jaagumagi, and Danny Shpeley travelled south and east through Sonora making collections near Nacozari de García, Huásabas, Río El Riíto, Rancho los Pinos, Mesa Trés Ríos, and Rio Bavispe before continuing eastward to Chuhuichupa in the state of Chihuahua. Then in 1986, George E. Ball, Frania, and Dean Mulyk travelled south along MEX 15, and collected near Peón (SE of Empalme), then continued to Sinaloa. Later that same year, they drove north from Sinaloa through Sonora, and made collections along MEX 15 near Hermosiin Nogales, on the Rio Magdalena, and near Hermosillo. Lastly, in 1993, George Ball, Kathleen Ball, and McCleve collected near Moctezuma, Santa Rosa and Yécora before driving east to Chihuahua. On the same trip, they also collected at El Llano (south of Santa Ana) and near Hermosillo on MEX 15 and near Álamos before driving south into Sinaloa.

In the last decade, there has been a concerted effort to document the biodiversity of the Sky Island mountain ranges in northeastern Sonora. From 2009 to 2019, there were major biotic inventories in 17 Sky Islands as part of the MABA and MDE programs. Smaller groups went to six additional Sky Islands plus numerous other areas throughout the state.

Interestingly, carabid specimens are recorded from 59 of the 72 municipalities in Sonora (Figure 9). The lack of carabid specimen records from the remaining 13 municipalities (Átil, Bácum, Benito Juárez, Etchojoa, Huepac, Oquitoa, San Ignacio Río Muerto, San Miguel de Horcasitas, San Pedro de la Cueva, Suaqui Grande, Tepache, Tubutama, and Villa Hidalgo) is an artifact of collecting rather than an absence of carabids in those municipalities. No attempts were made to visit each municipality in the state of Sonora to collect carabids.

\section{DISCUSSION}

There have been several publications of generic revisions or descriptions of new species of carabids known to occur in Mexico, and those papers usually include Sonora records (a few examples: Ball \& Shpeley 2002; Cazier 1954; Erwin 1970: Noonan 1973; Shpeley 1986; Shpeley \& Ball 1993; Whitehead 1972). A very recent paper by Messer \& Raber (2021) treated the genus Selenophorus north of Mexico, but also included many specimen records from Sonora and other states in Mexico. This is the first checklist of the carabids of Sonora, primarily based on specimens which were examined by the authors. Ball and Shpeley (2000) stated that the known carabid fauna of Mexico included 172 genera and 1,957 described species. The number of described species of carabids in Mexico today is probably more than 2,000. In this checklist, we recognized 92 genera and 388 taxa, of which 269 are described taxa and 119 are undescribed morphospecies. Bousquet (2012, Table 5) records 502 carabid species (in 107 genera) in Arizona and 646 carabid species (in 104 genera) in California. Based on the best information available, we estimate that the number of described carabid species in Sonora is approximately 500 , similar to that of Arizona, even though the number of Arizona genera is significantly higher. The total number of described carabid species is not available for Baja California or Sinaloa, but based on the best information available, we estimate that the number in Baja California would be less than that of Sonora, and the number in Sinaloa would be similar to that of Sonora.

Some of the taxa are represented by large numbers of specimens. Of the 388 taxa included in this study, two taxa are represented by over 500 specimens, and 38 taxa are represented by over 100 specimens. Most of the taxa are represented by fewer specimens. There are 179 taxa represented by 6 to 100 specimens, and 169 taxa are represented by 5 or fewer specimens. Which begs the question - are some of these species very common and some rare? Within the genus Brachinus, B. elongatulus is represented by 949 specimens, whereas the next most collected taxon in Brachinus is B. costipennis represented by 63 specimens. Many of the specimens collected are the results of single day events. Some of the single specimens for a taxon are the result of multiple day events in the same general area. Different methods of collecting can result in very different taxa collected. For example, many carabids are attracted to mercury vapor lights, but some carabids appear to only be attracted to blacklights.

As mentioned in the introduction, we do not claim this list to be the definitive number of species of carabids that may inhabit Sonora. This paper should be considered to be a starting point for a researcher interested in documenting and studying the carabid species in Sonora. Unfortunately, the lack of available revisions of many of the genera is the major stumbling block in applying published scientific names to many of the taxa collected.

\section{ACKNOWLEDGEMENTS}

The authors thank the Sky Island Alliance (Madrean Archipelago Biodiversity Assessment, MABA) and Great- 
er Good Charities (Madrean Discovery Expeditions, MDE) for providing the infrastructure to collect natural history specimens in the state of Sonora. We also thank the staff of the Bavispe Area for the Protection of Flora and Fauna, especially J. Mario Cirett-Galán, that accompanied the researchers on the collecting trips, not only setting up the camps but also as guides and preparing meals. We thank Drs. Nico Franz and Sangmi Lee for participating on several expeditions and curating MDE specimens in the Arizona State University Hasbrouck Insect Collection. We also thank Drs. Harry Brailovsky-Alperovitz and Alejandro Zaldívar-Riverón, Colección Nacional de Insectos, Universidad Nacional Autónoma de México for support under their SEMARNAT collecting permits

The first author thanks Tom Van Devender and Ana Lilia Reina-Guerrero for the hospitality extended to him at their home before and after each of the collecting trips in 2015, 2016, 2017 and 2018. The first author also thanks John D. Palting, who generously accepted the first author as a passenger on three of the collecting trips into Sonora. The first author thanks George E. Ball, who was extremely supportive of this project, and personally covered most of the author's expenses for data collection visits to the California Academy of Sciences (San Francisco, California) and the National Museum of Natural History (Washington, D.C.). The first author thanks David R. Maddison who identified some of the Bembidion species. The first author also thanks Peter Messer for his constructive comments that improved the manuscript. Lastly, the first author also thanks Martin Bierens, Kyla Gupta, and Kenmir Boyd who did data entry of some of the carabid specimens collected in Sonora.

The second author thanks the many biologists from Mexico and the United States who went on the MDE and MABA Expeditions. Ed Gilbert created the MDE (madreadiscovery.org) and MABA databases in the SEINet network where the Sonoran carabid records are publicly available. Ana Lilia Reina-Guerrero was a stalwart companion on all expeditions and other Sonoran travels. John D. Palting, moth specialist extraordinaire, facilitated most collecting efforts, especially with his ultraviolet lights at each camp. The unflagging support of Elizabeth Baker, Noah Horton, Brooke Nowak, and Steve Minter at Greater Good Charities for the MDE biodiversity programs in the Sky Island mountains of Sonora is greatly appreciated.

\section{ANNOTATED CHECKLIST OF SONORA CARABIDS}

This checklist follows the names and arrangement of tribes as published by Bousquet (2012). The one exception is the tribe name Manticorini, used by Wiesner (2020), whereas Bousquet (2012) used the tribe name Amblycheilini. In this checklist, the tiger beetles are included as members of the family Carabidae (Bousquet, 2012). The scientific names of tiger beetles have been updated to the list published by Wiesner (2020). Genera, species and subspecies are arranged alphabetically for ease of location in the manuscript.

All of the headings in the following checklist are self-explanatory, and are based on specimens seen and their associated label data. Tribes, in bold font, are numbered from 01 to 35. Taxa are numbered from 001 to 388, in normal font (not bold). Some of the taxa scientific names include the collection acronym in which they are housed (i.e., CASC, UASM and USNM). Non-label vegetation data has also been added to the Vegetation heading when this information was known by the authors. Elevation for a few well-known localities has been added when not present on the specimen locality labels. Note that "at light", "at uv light", and "at $\mathrm{Hg}$ vapor light" are included under the Habitat heading as [at light]. 


\section{Class Insecta \\ Order Coleoptera \\ Family Carabidae \\ 01. Notiophilini}

001. Notiophilus chihuahuae Casey, 1913

Number examined: 6 .

Municipalities: Huachinera, Nacozari de García.

Habitat: north-south rocky ridge top

Vegetation: meadow, pine-oak forest.

Elevation range: $2090-2337 \mathrm{~m}$.

Collection dates: 1982.VIII.04; 2015.VIII.10.

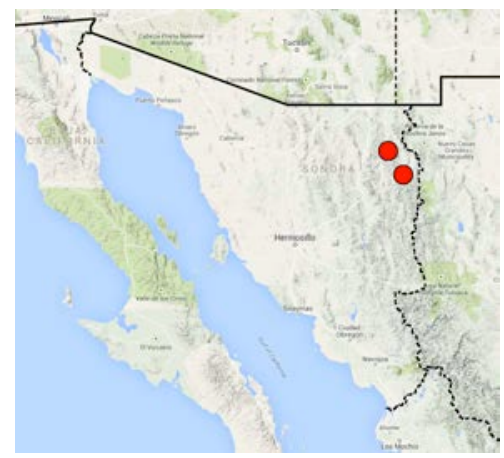

002. Notiophilus semiopacus Eschscholtz, 1833

Number examined: 2 .

Municipality: Huachinera.

Habitat:?

Vegetation: meadow, pine-oak forest.

Elevation: $2090 \mathrm{~m}$.

Collection date: 1982.VIII.04.

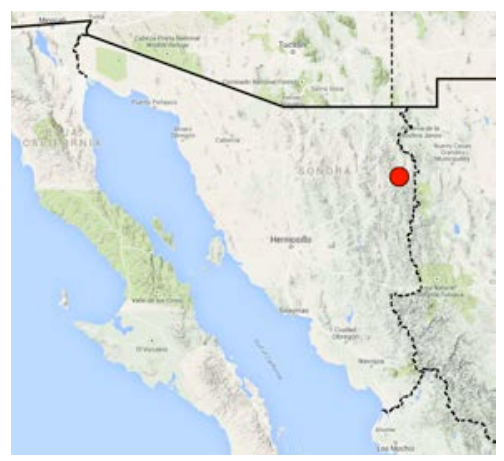

\section{Cychrini}

003. Scaphinotus mexicanus Bates, 1882

Number examined: 5 .

Municipalities: Huachinera, Nácori Chico.

Habitat: ?

Vegetation: meadow, pine-oak forest.

Elevation range: $1950-2330 \mathrm{~m}$.

Collection dates: 1982: VIII.04, VIII.06-VIII.08.

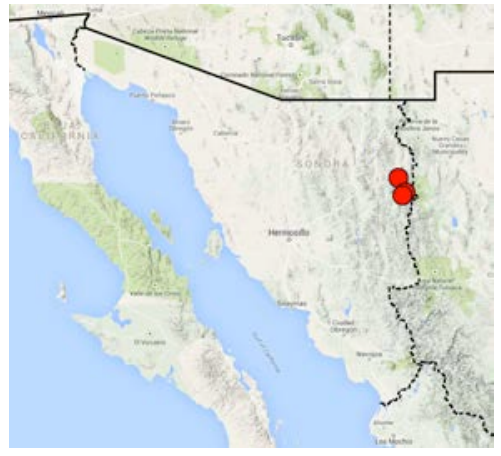

\section{Carabini}

004. Carabus forreri Bates, 1882

Number examined: 6 .

Municipalities: Nácori Chico, Nacozari de García.

Habitat: rocky stream canyon

Vegetation: cypress-maple-alder-sycamore riparian forest; pine-oak forest on slope

Elevation range: $1715-2278 \mathrm{~m}$.

Collection dates: 1982: VIII.06-VIII.07; 2015.VIII.11; 2018.VI.27.

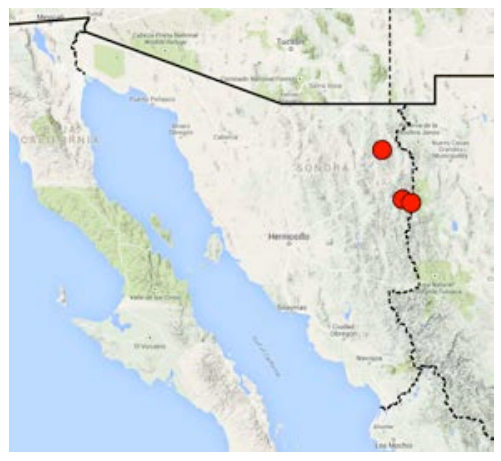

005. Calosoma angulatum Chevrolat, 1834

Number examined: 186.

Municipalities: Aconchi, Álamos, Bacanora, Bacoachi, Benjamín Hill, Cajeme, Fronteras, Granados, Hermosillo, Huásabas, La Colorada, Magdalena de Kino, Mazatán, Moctezuma, Naco, Nácori Chico, Nacozari de García, Rosario, Rayón, Sahuaripa, San Felipe de Jesús, Yécora.

Habitat: gentle granitic slope; rocky mountainside; rocky stream canyon; stream margin; motel; urban sidewalks; at light.

Vegetation: Chihuahuan desertscrub; desert grassland; cypress-maple-alder-sycamore riparian forest; foothills thornscrub; oak woodland; pine-oak forest; riparian tropical scrub; Sonoran desertscrub; tropical decidous forest.

Elevation range: $396-1687 \mathrm{~m}$.

Collection dates: 1940.VII.29; 1953: VII.09-VII.16; 1957: VII.28-VII.29; 1958.VIII.01; 1963.II.20; 1965. VII; 1969: VII.16-VII.17; 1971.VIII.16; 1979.VIII.23; 1980: IX.17-IX.18; 1982: VIII.01, VIII.11; 1984. VIII.24; 1987: VII.26-VII.31; 1993: VII.09, VII.14; 
2012: VII.12, VIII.01, VIII.03, VIII.04, VIII.06; IX.02, IX.05; 2013.VII.16; 2014: 07.28, VIII.02, VIII.29; 2015: VII.12, VIII.08; 2017: VII.22-VII.23, VIII.13VIII.16, IX.02; 2018: VIII.05-VIII.07, VIII.09, VIII.11; 2019: VII.22, VII.24, VIII.05-VIII.06, IX.26.

Note: Seven specimens were dug out of rocky soil about 25 $\mathrm{cm}$ below the surface on a steep rocky mountainside in oak woodland.

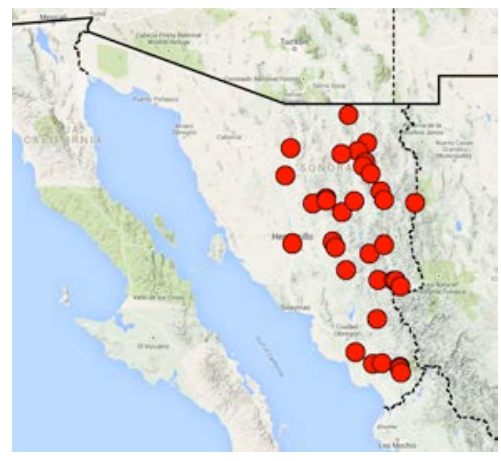

006. Calosoma parvicolle Fall, 1910

Number examined: 26 .

Municipalities: Hermosillo, General Plutarco Elías Calles, Guaymas, Pitiquito, Puerto Peñasco, San Luis Río Colorado.

Habitat: disturbed area; sandy flats and sand dunes.

Vegetation: coastal Sonoran desertscrub; coastal Sonoran dunescrub.

Elevation range: $2-42 \mathrm{~m}$.

Collection dates: 1958.IV.02; 1966.IV.24; 1973.V; 2017: II.27, III.17-III.18; 2019.III.05.

Note: Gidaspow (1959) recorded this species from La Choya (municipality of Puerto Peñasco, mapped) and Puerto Libertad (municipality of Pitiquito, mapped) on the west coast.

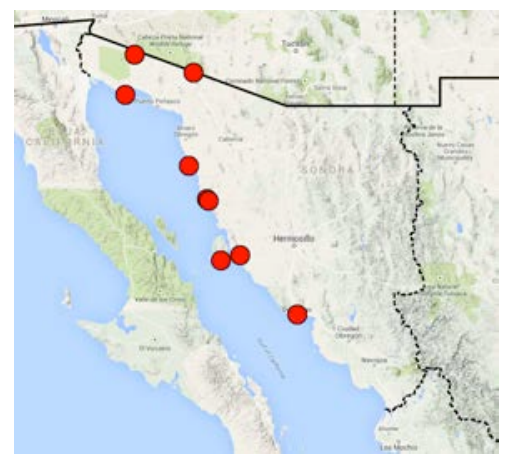

007. Calosoma peregrinator Guérin-Méneville, 1844

Number examined: 86.

Municipalities: Álamos, Aconchi, Agua Prieta, Bacadéhuachi, Benjamín Hill, Cananea, Carbó, Cucurpe, Empalme, Fronteras, Hermosillo, Ímuris, Magdalena de Kino, Moctezuma, Naco, Nacozari de García, Nogales, Opodepe, San Felipe de Jesús, Santa Ana, Trincheras.

Habitat: rocky slopes, ridge, canyon, arroyo; PEMEX sta- tion; urban. [at light]

Vegetation: Chihuahuan and Sonoran desertscrub; desert grassland; foothills thornscrub; pine-oak forest; riparian tropical scrub; sycamore riparian deciduous forest oak woodland; tropical deciduous forest.

Elevation range: $7-2467 \mathrm{~m}$.

Collection dates: 1950.VIII.06; 1953: VII.09-VII.16; 1960. VIII.08; 1963: II.20, VII.06, VIII.06; 1980: VII.21-22, IX.18-IX.19; 1982: VIII.01, VIII.09, VIII.23; 1986. VIII.05; 1990.IX.03; 1993.VII.14; 2012: VII.30, VII.21, VIII.01, IX.02, IX.04, IX.05, IX.11, IX.12; 2013: VII.17, VIII.02, VIII.04, VIII.23, IX.08 - IX.11; 2014: VIII.25, VIII.28, IX.14, IX.16; 2015: VII.12, VIII.08, VIII.10, VIII.13, IX.06; 2016: VIII.13-VIII.15, VIII.24; 2017: VII.16, VII.23-VII.24, VIII.14-VIII.16, IX.02-IX.03; 2019.VIII.05.

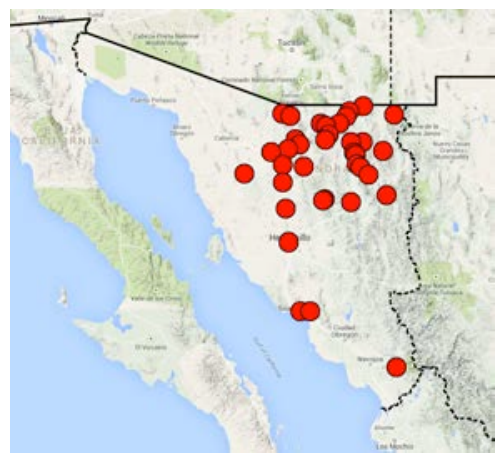

008. Calosoma prominens LeConte, 1853

Number examined: 142 .

Municipalities: Álamos, Agua Prieta, Benjamín Hill, Cananea, Carbó, Empalme, Fronteras, Granados, Guaymas, Hermosillo, Magdalena de Kino, Mazatán, Nogales, Opodepe, Santa Ana.

Habitat: gentle granitic slopes; PEMEX station at night; urban. [at light]

Vegetation: Chihuahuan and Sonoran desertscrub; coastal thornscrub; desert grassland; foothills thornscrub; oak woodland; tropical deciduous forest.

Elevation range: $7-1587 \mathrm{~m}$.

Collection dates: 1953: VII.07-VII.16, VIII.15-VIII.20, VIII.27, IX.28; 1954.VIII.24; 1955.VIII.21; 1957. VIII.16; 1959.VIII.12; 1960.VIII.08; 1961.VIII.7; 1963: II.20, VII.06; 1965.VII.31; 1967.VII.27; 1982. VIII.23; 1970.VIII.20; 1971.VIII.20; 1986.VIII.05; 2012: VIII.08, IX.05, IX.10, IX.11; 2013: III.18, VIII.09, VIII.10, VIII.23; 2014.VII.28; 2016.VIII.24; 2017: VII.21-VII.23, IX.17; 2018.VIII.11. 


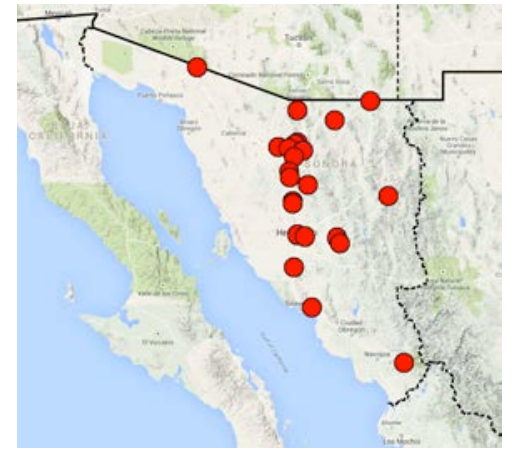

009. Calosoma protractum LeConte, 1862

Number examined: 2 .

Municipality: Huachinera.

Habitat: ?

Vegetation: desert grassland.

Elevation: $1150 \mathrm{~m}$.

Collection date: 1982.VIII.05.

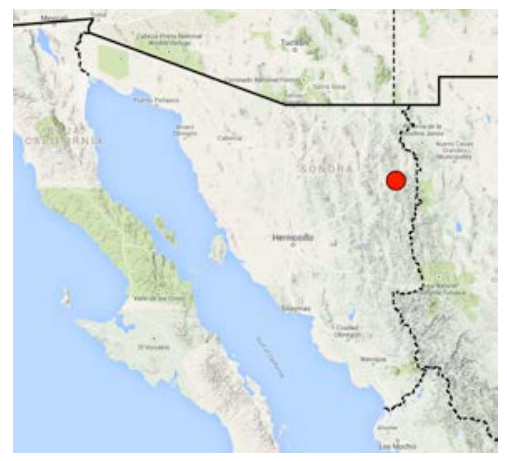

010. Calosoma sayi Dejean, 1826

Number examined: 5 .

Municipalities: Álamos, Empalme.

Habitat: Urban.

Vegetation: coastal thornscrub; tropical deciduous forest;

Elevation range: $7-400 \mathrm{~m}$.

Collection dates: 1986.VIII.05; 2012: IX.11, IX.12.

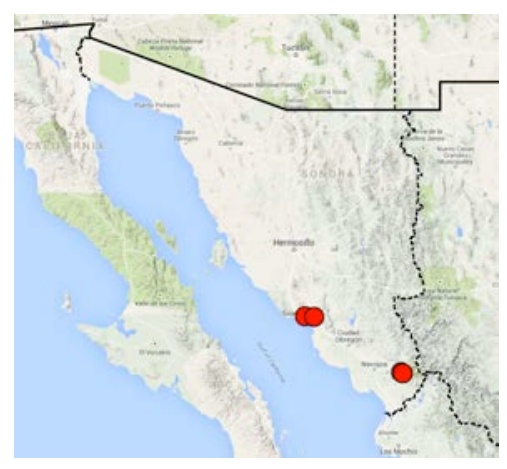

011. Calosoma scrutator (Fabricius, 1775)

\section{Number examined: 49.}

Municipalities: Aconchi, Álamos, Cananea, Fronteras, Magdalena de Kino, Mazatán, Moctezuma, Nácori Chico, Nacozari de García, Nogales, San Felipe de Jesús,
Santa Cruz, Sáric, Yécora.

Habitat: gentle granitic slopes; rocky arroyo, canyon, hillside and mountainside; urban. [at light]

Vegetation: cypress-maple-alder-sycamore riparian forest; desert grassland; foothills thornscrub; oak woodland; pine-oak forest; riparian tropical scrub; Sonoran desertcrub; tropical deciduous forest.

Elevation range: $780-2422 \mathrm{~m}$.

Collection dates: 1929.VIII.22; 1940.VIII.30; 1982: VIII.01, VIII.11; 1990: VI.30-VII.03; 2012: IX.02, IX.03; 2013: III.20, VIII.02, VIII.09, IX.08, IX.09; 2014: VIII.25, IX.14, IX.16; 2016.VII.14; 2017: VIII.14-VIII.15, IX.17; 2018: VIII.07-VIII.08; 2019: IV.24-IV.25, VIII.05.

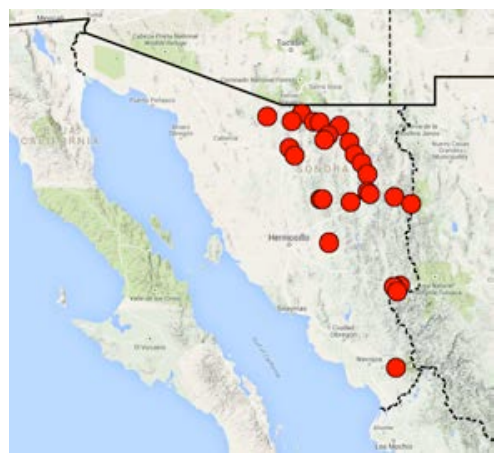

012. Calosoma sponsum Casey, 1897

Number examined: 1.

Municipality: Hermosillo.

Habitat: Urban.

Vegetation: Sonoran desertscrub.

Elevation:?

Collection date: 1958.IV.04.

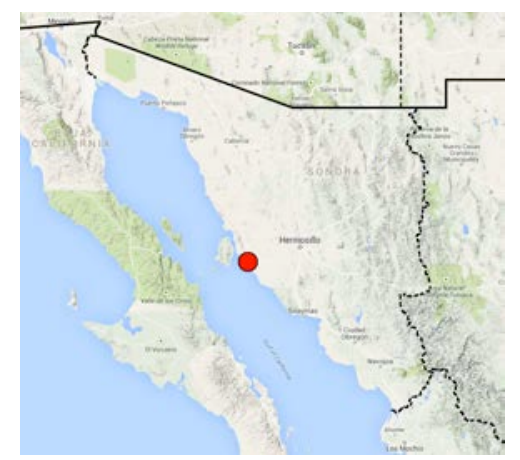

04. Amblycheilini

013. Amblycheila baroni Rivers, 1890

Number examined: 8.

Municipalities: Agua Prieta, Arizpe, Bacoachi, Cananea, Nacozari de García.

Habitat: rocky ridge; rocky slope; rocky stream canyon.

Vegetation: Chihuahuan desertscrub; desert grassland; oak woodland; riparian forest.

Elevation range: $750-2467 \mathrm{~m}$. 
Collection dates: 1998.VIII.05; 2009.IX.07; 2013: VII.17, VIII.02, IX.09.

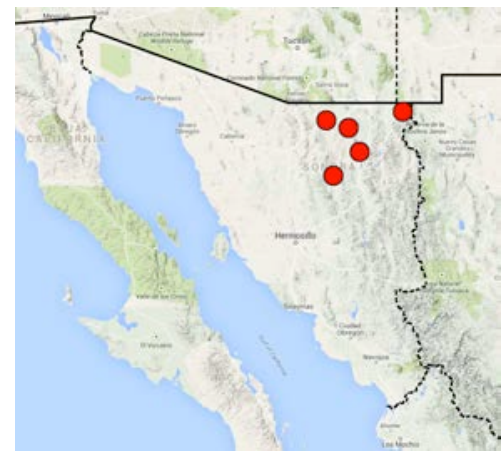

\section{Megacephalini}

014. Tetracha carolina Linnaeus, 1767

Number examined: 109.

Municipalities: Álamos, Cajeme, Guaymas, Hermosillo, Ímuris, Mazatán, Moctezuma, Nacozari de García, Navojoa, Pitiquito, Santa Ana.

Habitat: urban. [at light]

Vegetation: coastal and foothills thornscrub; riparian tropical scrub; Sonoran desertscrub; tropical deciduous forest.

Elevation range: $255-707 \mathrm{~m}$.

Collection dates: 1940.VII.29; 1950.VII.15; 1953: VII.09VII.16, VIII.01-VIII.15, VIII.20-VIII.31, IX.01-IX.10; 1960: VII.27, VIII.10; 1963.VII.14; 1965.VII.31; 1967: VI.12, VII.27; 1985.VII.31; 2013.IX.09; 2017: VII.21, VII.23, IX.08.

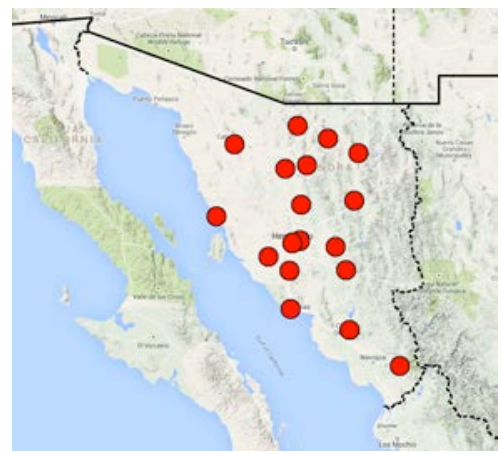

\section{Cicindelini}

015. Brasiella wickhami (W. Horn, 1903)

Number examined: 303.

Municipalities: Agua Prieta, Álamos, Cajeme, Carbó, Empalme, Fronteras, Guaymas, Hermosillo, Mazatán, Moctezuma, Nacozari de García, Navojoa, Ónavas, Opodepe, Santa Ana.

Habitat: cow pasture; rocky arroyo. Urban [at light]

Vegetation: Chihuahuan and Sonoran desertscrub; coastal and foothills thornscrub; oak woodland; tropical deciduous forest.

Elevation range: 3 - $1429 \mathrm{~m}$.

Collection dates: 1950.VIII.10; 1952.VIII.02; 1953:
VII.09-VII.16, VIII.15-VIII.20; 1954.VIII.24; 1959. VIII.29; 1960: VI.27, VII.15, VII.27, VIII.08-VIII.10, VIII.12; 1963.VIII.06; 1965.VII.31; 1967.VI.27; 1979: VII.07-VII.08; 1980.IX.16; 1982: VIII.19-VIII.20, VIII.23; 1986.VIII.29; 1987: VII.26-VII.27, VII.30VII.31; 2016.VII.02; 2017: VII.22-VII.23, VIII.14; 2018.VIII.09; 2019: VII.24, VII.26.

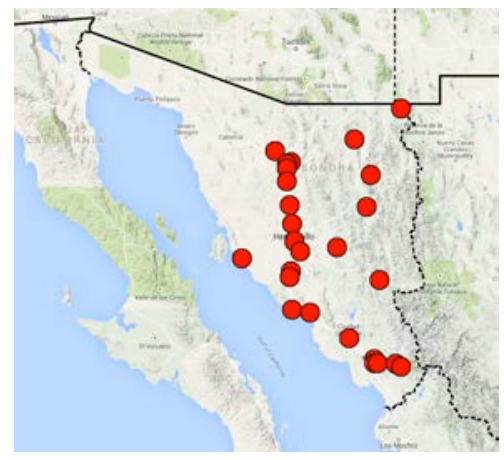

016. Brasiella viridisticta arizonensis (Bates, 1884)

Number examined: 26.

Municipalities: Álamos, Cananea, Fronteras, Moctezuma, Nácori Chico, Ímuris, Santa Ana, Yécora.

Habitat: cow pasture; marsh; river. [at light]

Vegetation: desert grassland; foothills thornscrub; oak woodland; pine-oak forest; Sonoran desertscrub; tropical deciduous forest

Elevation range: $518-1950 \mathrm{~m}$

Collection dates: 1967.VII.27; 1984.VIII.26; 1985: VIII.01, VIII.02; 2016.VII.02; 2017.VII.16.

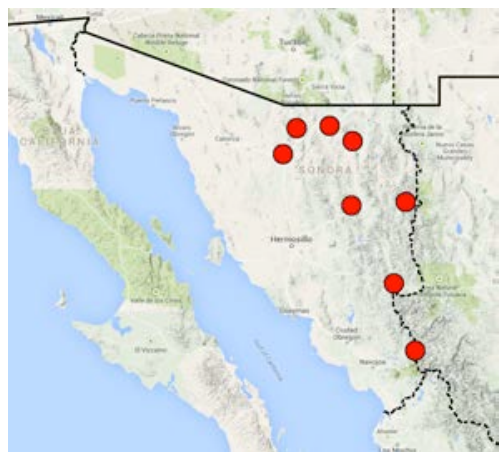

017. Cicindelidia aeneicollis (Bates, 1881)

Number examined: 2 .

Municipalities: Álamos, Yécora.

Habitat: ?

Vegetation: Pine-oak forest; tropical deciduous forest.

Elevation range: $400-1547 \mathrm{~m}$.

Collection dates: 1958: VII.15-VII.20; 1990: VI.30-VII.1. 


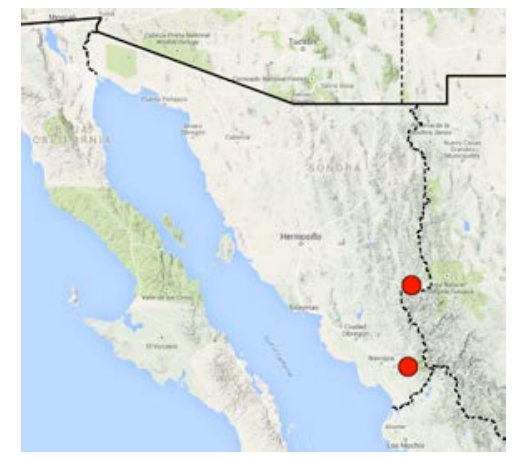

018. Cicindelidia beneshi (Varas-Arangua, 1930)

Number examined: 353.

Municipality: Puerto Peñasco.

Habitat: mud flats; salt marsh flats; sandy beach.

Vegetation: coastal saltscrub.

Elevation: ?

Collection dates: 1952.VI.12; 1954.V.30; 1966: VII.17, VII.29-VII.30; 1971.VII.27; 1972.V.16; 1987: V.15, V.31, VI.01; 1991: VII.15, VII.16; 1995: V.28, VI.05.

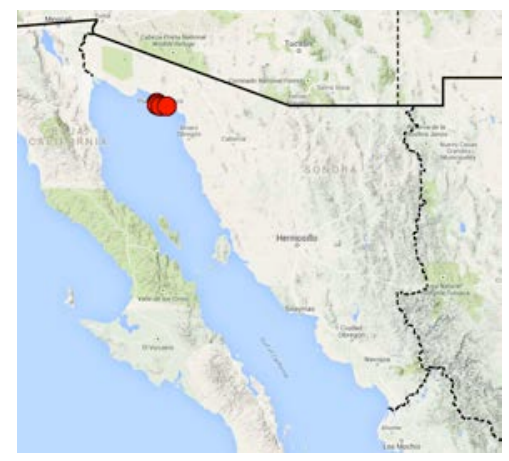

019. Cicindelidia carthagena carthagena (Dejean, 1831) Number examined: 24.

Municipalities: Empalme, Guaymas.

Habitat: ?

Vegetation: coastal thornscrub; Sonoran desertscrub.

Elevation: ?

Collection dates: 1952.VII.25; 1954.VII.10; 1966: VI.01VI.02, VII.21.

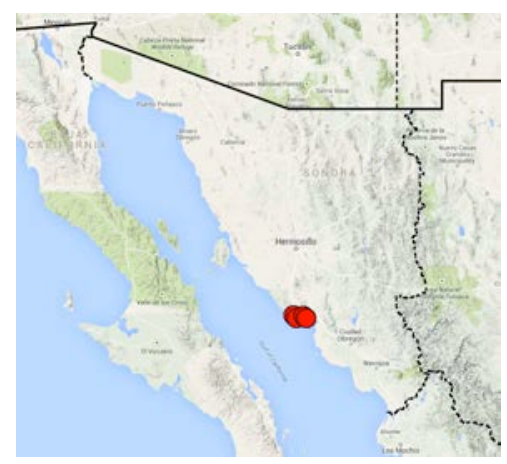

020. Cicindelidia carthagena colossea (W. Horn, 1926) Number examined: 17.

Municipality: Pitiquito.

Habitat: ?

Vegetation: Sonoran desertscrub

Elevation: ?

Collection dates: 1953.VIII.11; 1975.VII.10.

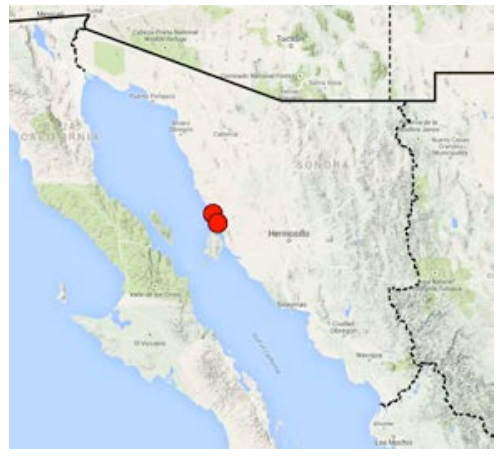

\section{Cicindelidia carthagena carthagena X carthagena} colossea

Number examined: 8.

Municipality: Hermosillo

Habitat: tidal flat.

Vegetation: coastal saltscrub.

Elevation: ?

Collection dates: 1958.X.24; 1966.VII.19.

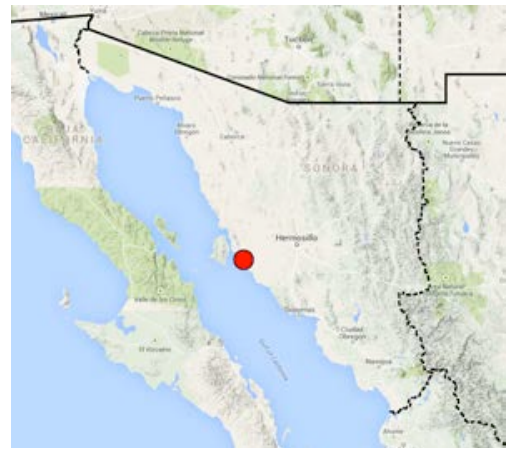

022. Cicindelidia fera (Chevrolat, 1834)

Number examined: 23.

Municipalities: Álamos, Altar, Hermosillo, Pitiquito.

Habitat: ?

Vegetation: Sonoran desertscrub; tropical deciduous forest. Elevation: ?

Collection dates: 1897.IV.19; 1934.XII.01; 1952.VII.04; 1953: V.27, VII.09-VII.16 


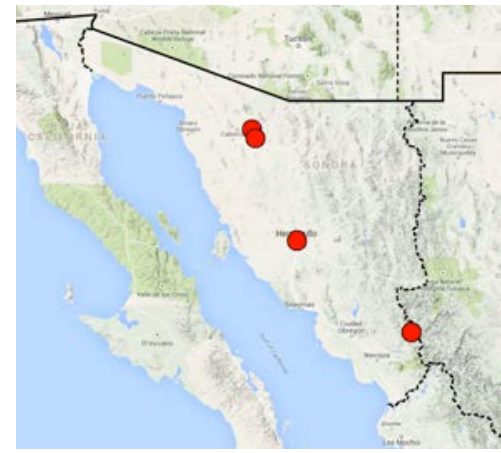

023. Cicindelidia haemorrhagica haemorrhagica (LeConte, 1851)

Number examined: 4.

Municipality: San Luis Río Colorado.

Habitat: ?

Vegetation: Sonoran desertscrub.

Elevation: ?

Collection dates: 1949: VI.04-VI.05.

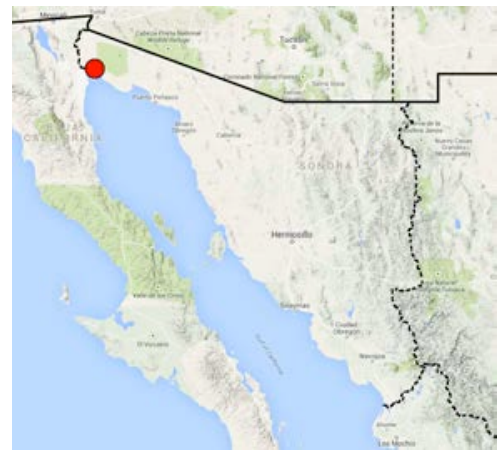

024. Cicindelidia juvenilis (W. Horn, 1897)

Number examined: 2.

Municipality: Huatabampo.

Habitat: ?

Vegetation: coastal thornscrub.

Elevation: $46 \mathrm{~m}$.

Collection date: 1967.VII.28.

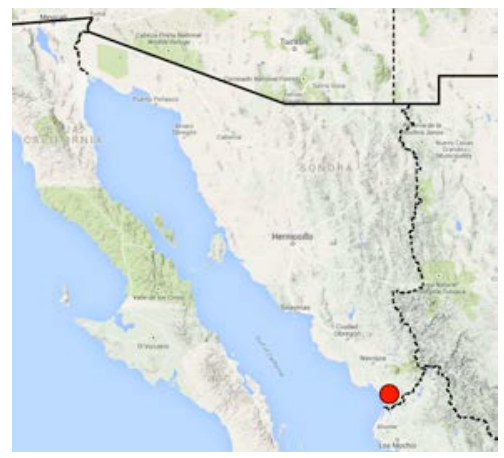

025. Cicindelidia latesignata parkeri (Cazier, 1848)

Number examined: 49.

Municipalities: Caborca, Puerto Peñasco.

Habitat: ?

Vegetation: Sonoran desertscrub.

Elevation: ?

Collection dates: 1941.IV.20; 1949.III.27; 1952.VI.12; 1960.IV.07; 1966: V.29-V.30; 1990.IV.07.

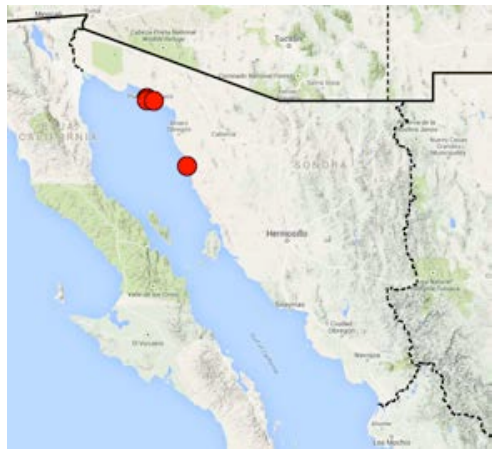

026. Cicindelidia melissa Duran \& Roman, 2014

Number examined: 10

Municipality: Nácori Chico.

Habitat: steep mountainside, along roadside; stream margin.

Vegetation: pine-oak forest.

Elevation range: $1893-2080 \mathrm{~m}$.

Collection dates: 1983.VII.04; 2018.VIII.07

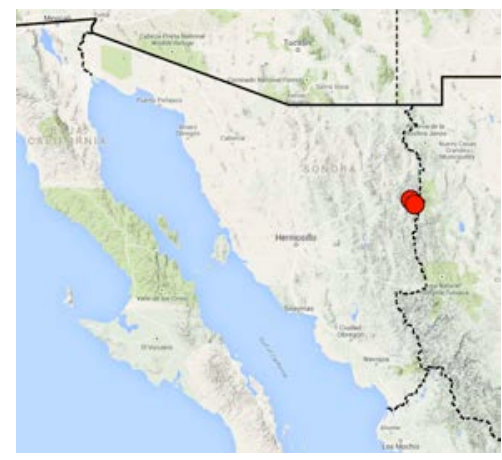

027. Cicindelidia obsoleta santaclarae (Bates, 1890)

Number examined: 3 .

Municipality: Fronteras.

Habitat: cow pasture.

Vegetation: oak woodland.

Elevation: $1429 \mathrm{~m}$.

Collection date: 2016.VII.02. 


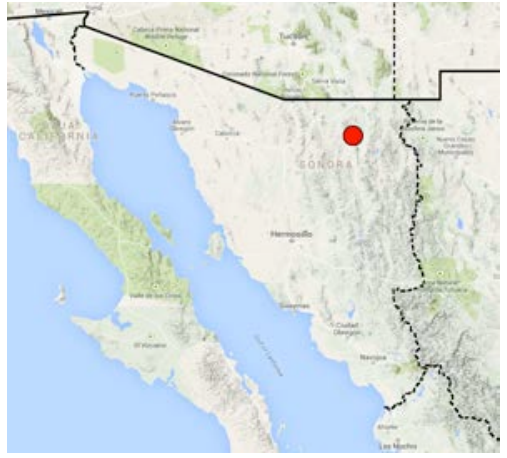

028. Cicindelidia ocellata (Klug, 1834)

Number examined: 99 .

Municipalities: Álamos, Fronteras, Nácori Chico, Nogales, Santa Ana, Ures.

Habitat: cow pasture; rocky mountainside; rocky stream canyon. [at light]

Vegetation: cypress-maple-alder-sycamore riparian forest; oak woodland; pine-oak forest.

Elevation range: $1429-1715 \mathrm{~m}$.

Collection dates: 1966.VII.18; 1983.VII.04; 1985.VIII.01; 2016: VII.02, VIII.15; 2018.VI.27.

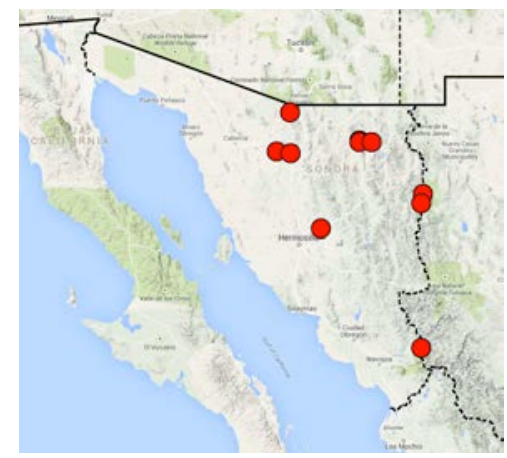

029. Cicindelidia sedecimpunctata (Klug, 1834)

Number examined: 171.

Municipalities: Aconchi, Álamos, Bacanora, Banámichi, Cucurpe, Cumpas, Fronteras, Ímuris, Magdalena de Kino, Moctezuma, Nacozari de García, Nogales, Ónavas, Santa Ana, Ures, Yécora.

Habitat: rocky mountainside. [at light]

Vegetation: Chihuahuan and Sonoran desertscrub; foothills thornscrub; oak woodland; pine-oak forest; riparian tropical scrub; tropical deciduous forest.

Elevation range: $244-1687 \mathrm{~m}$.

Collection dates: 1940: VII.28, VII.30, VIII.01; 1952: VII.02, VII.19; 1953: VII.09-VII.16, IX.14; 1958. VII.08; 1960.VIII.12; 1963: VII.13, VII.15; 1966: VII.18, VII.22; 1982.VIII.01; 1987: VII.26-VII.31; 2017: VII.23, VIII.14, VIII.16; 2019: VI.03, VII.24.

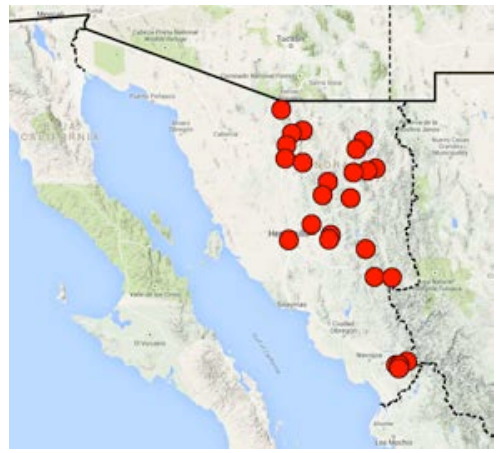

030. Cicindelidia sommeri (Mannerheim, 1837)

Number examined: 208 .

Municipalities: Álamos, Altar, Hermosillo, Ímuris, Moctezuma, Nacozari de García, Pitiquito, Ures.

Habitat: sandy river bottom.

Vegetation: Sonoran desertscrub; foothills thornscrub; oak woodland; tropical deciduous forest.

Elevation range: 244 - $1000 \mathrm{~m}$.

Collection dates: 1897.IV.19; 1934.XII.01; 1952.VII.04; 1953: IX.14-IX.15; 1955.XI.12; 1963: VII.13, VII.15; 1966: VII.18, VII.22; 1993.VII.04.

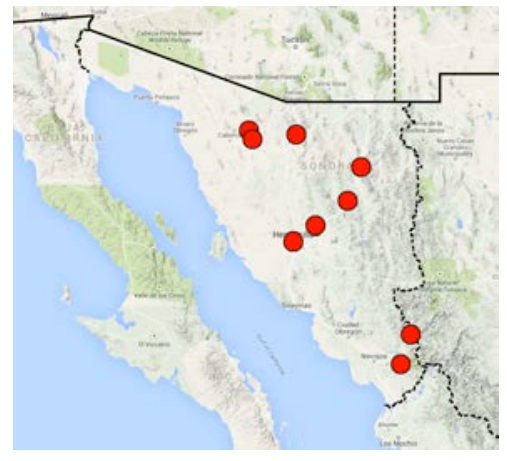

031. Cicindelidia tenuisignata (LeConte, 1851)

Number examined: 5.

Municipality: Cajeme.

Habitat: ?

Vegetation: coastal thornscrub.

Elevation:?

Collection dates: 1952.VII.29; 1953.IX.15.

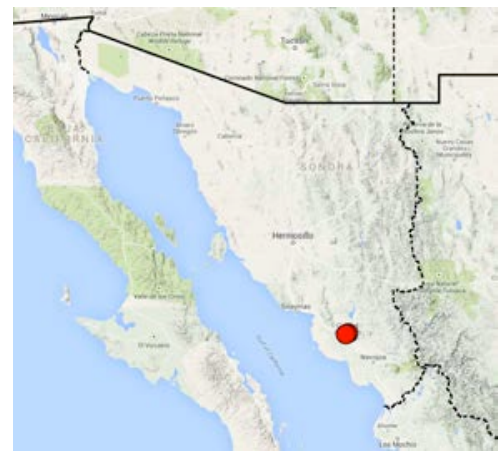




\section{Cicindelidia thalestris (Bates, 1890)}

Number examined: 1 .

Municipality: Aconchi.

Habitat: ?

Vegetation: pine-oak forest.

Elevation: $1860 \mathrm{~m}$.

Collection date: 2012.VII.07.

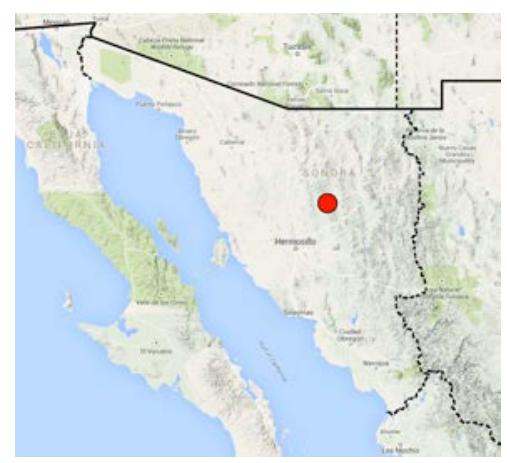

033. Cicindelidia trifasciata ascendens (LeConte, 1851) Number examined: 35 .

Municipalities: Empalme, Guaymas, Hermosillo.

Habitat: tidal flat.

Vegetation: coastal thornscrub; Sonoran desertscrub.

Elevation:?

Collection dates: 1966: VI.02, VII.19, VII.21; 1973.V.

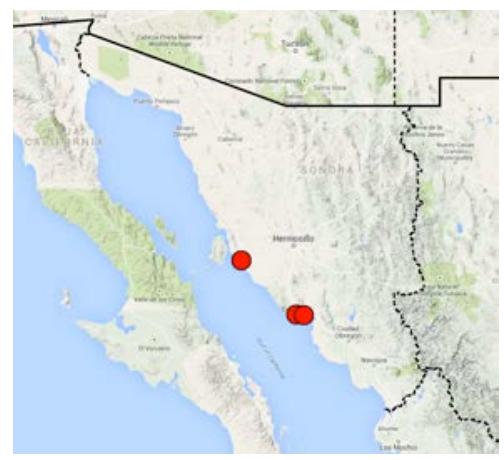

034. Cicindelidia sp. near thalestris (Bates, 1890)

Number examined: 2 .

Municipality: Álamos.

Habitat: [at light]

Vegetation: tropical deciduous forest.

Elevation: $1577 \mathrm{~m}$.

Collection dates: 1989: VII.11-VII.13.

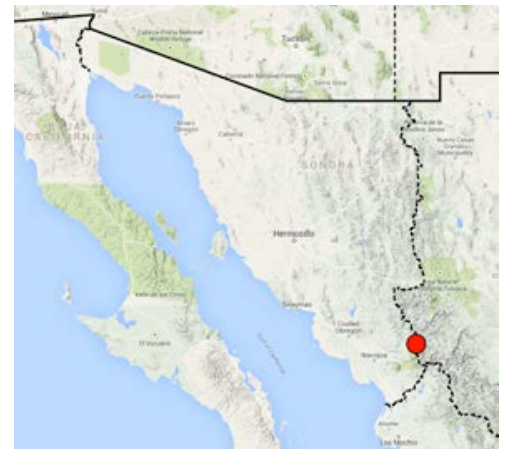

035. Cylindera lemniscata lemniscata (LeConte, 1854)

Number examined: 233.

Municipalities: Agua Prieta, Álamos, Caborca, Cajeme, Cananea, Carbó, Empalme, Fronteras, General Plutarco Elías Calles, Guaymas, Hermosillo, Ímuris, Magdalena de Kino, Navojoa, Nogales, Opodepe, Pitiquito, Puerto Peñasco, Santa Ana, Trincheras.

Habitat: cow pasture; river bottom; urban. [at light]

Vegetation: desert grassland; oak woodland; Sonoran desertscrub.

Elevation range: $3-1521 \mathrm{~m}$.

Collection dates: 1940.VII.22; 1950: VII.14-VII.15, VIII.06, VIII.10; 1953: VII.09-VII.31, VIII.27, IX.01IX.10, IX.26; 1954.VIII.24; 1958: VII.11, VII.16; 1960: VII.21, VII.27-VII.28, VIII.07-VIII.08, VIII.10, VIII.12; 1965: VII.30, VII.31; 1966.VII.17; 1979: VII.07-VII.08, VIII.20; 1982: VIII.19-VIII.20, VIII.23; 1985: VIII.02, VIII.05; 2015: VII.12, VIII.08, IX.06; 2016.VII.02; 2017: VII.21, VII.23; 2018.VIII.13.

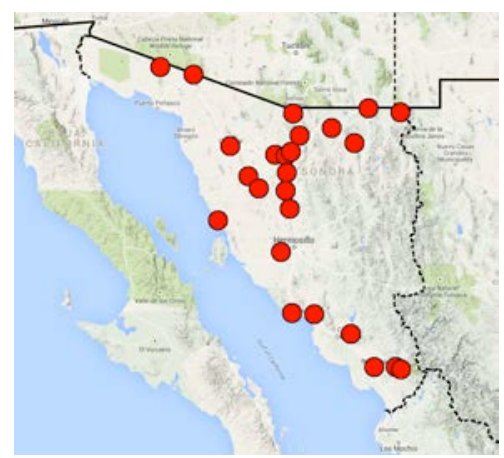

036. Ellisoptera nevadica nevadica (LeConte, 1875)

Number examined: 7.

Municipality: Puerto Peñasco.

Habitat: open sand flats. [at light]

Vegetation: creosotebush.

Elevation:

Collection date: 2019.V.04.

Note: Cazier (1954) recorded only 2 specimens of this species from La Choya (municipality of Puerto Peñasco, mapped) on the west coast. 


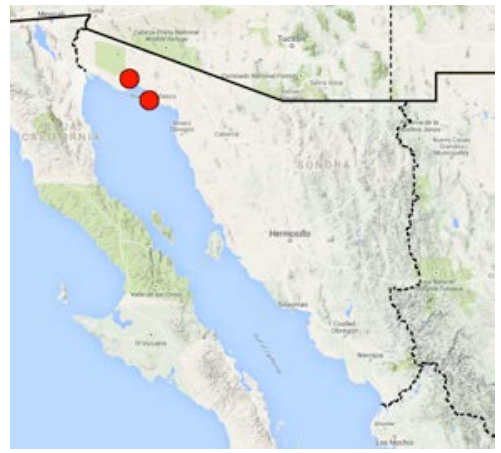

037. Ellisoptera sperata vauriei (Cazier, 1954)

Number examined: 5.

Municipalities: Hermosillo, Pitiquito.

Habitat: ?

Vegetation: Sonoran desertscrub.

Elevation: ?

Collection dates: 1952.VII.04; 1958.VII.22.

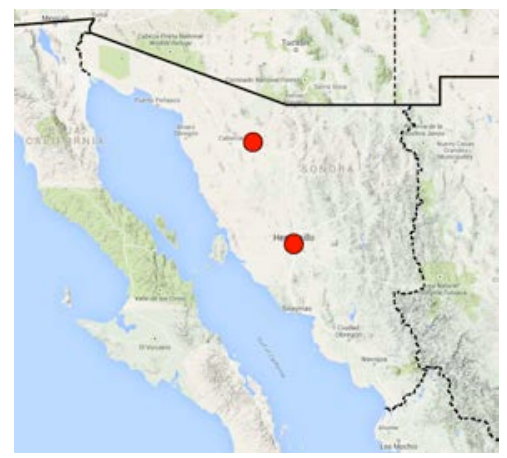

038. Eunota californica brevihamata (W. Horn, 1908)

Number examined: 139.

Municipalities: Guaymas, Hermosillo, Huatabampo, Opodepe.

Habitat: tidal flat.

Vegetation: coastal saltscrub; Sonoran desertscrub.

Elevation: $3 \mathrm{~m}$.

Collection dates: 1950.VII.14; 1952.VII.31; 1953: VII.04VII.06; 1958.VII.13; 1960.VIII.10; 1961.VI.07; 1966: VII.19, VII22; 1970.VIII.23; 1971: VII.21-VII.22; 1979: VII.07-VII.08.

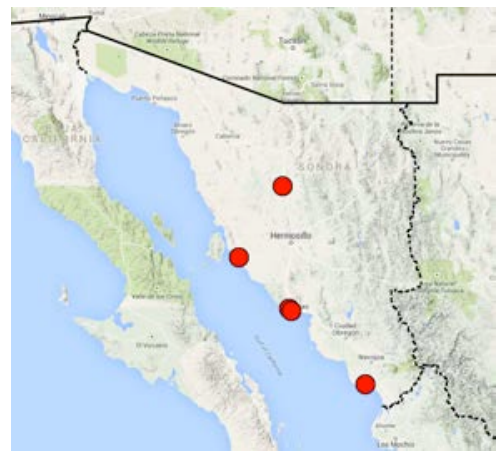

039. Eunota californica mojavi (Cazier, 1937)

Number examined: 250.

Municipalities: Pitiquito, Puerto Peñasco.

Habitat: coastal dunes; mud flats; salt marsh flats; tidal salt flats. [at light]

Vegetation: coastal saltscrub.

Elevation: $5-10 \mathrm{~m}$.

Collection dates: 1952: VI.12, VIII.16; 1953.VIII.11; 1966: V.29-V.30, VII.17; 1980.VIII.22; 1987: V.31, VI.01; 1991: VII.15-VII.16, VIII.28, VII.31, IX.01; 1994: VIII.01-VIII.02; 1995.VI.05; 2018.VIII.16.

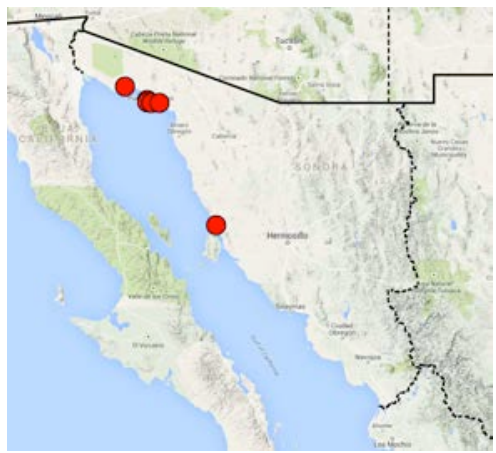

040. Eunota gabbii (G. Horn, 1866)

Number examined: 167

Municipalities: Caborca, Empalme, Hermosillo, Puerto Peñasco.

Habitat: coastal dunes; sandy beach; tidal flat; tidal salt flat. [at light]

Vegetation: coastal saltscrub.

Elevation: ?

Collection dates: 1952.VII.18; 1966: VI.01, VII.17, VII.19; 1967: V.31, VI.01, VII.16; 1991: VIII.31, IX.01; 1994: VIII.01-VIII.02; 2018.VIII.16. One label reads only "Oct.".

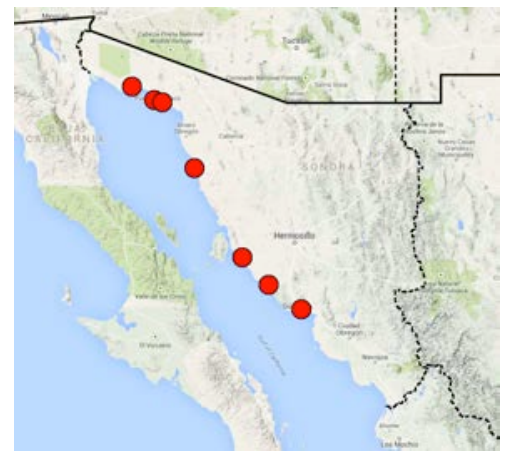

041. Eunota rockefelleri (Cazier, 1954)

Number examined: 8 .

Municipality: Puerto Peñasco.

Habitat: ?

Vegetation: coastal saltscrub.

Elevation:

Collection dates: 1952.VI.12; 1966.V.30. 


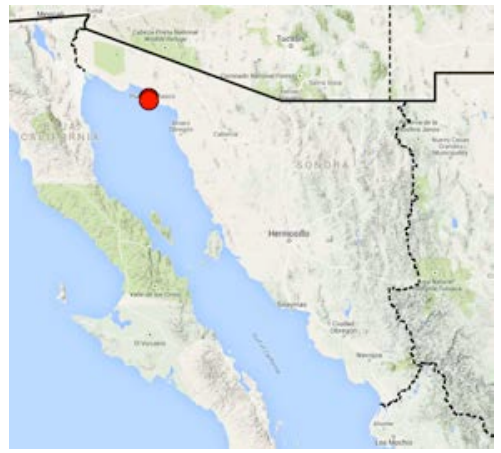

042. Microthylax digueti (W. Horn, 1897)

Number examined: 10.

Municipality: Hermosillo.

Habitat: ?

Vegetation: Sonoran desertscrub.

Elevation: ?

Collection dates: 1950-VII-25; 1952.VII.13.

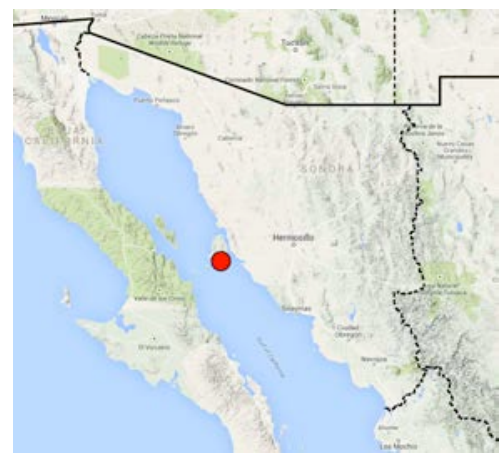

043. Microthylax sinaloae schrammeli (Cazier, 1954)

Number examined: 140.

Municipalities: Hermosillo, Pitiquito, Puerto Peñasco.

Habitat: coastal dunes; mud flats; sandy beach; salt marsh flats; tidal salt flats. [at light]

Vegetation: coastal saltscrub.

Elevation: $10 \mathrm{~m}$.

Collection dates: 1950.VII.25; 1952: VI.12, VII.16; 1953: VII.17-VII.31，VIII.01-VIII.15，IX.01-IX.10; 1966: V.30, VII.17; 1967.V.31; 1968.VII; 1970.VIII.22; 1975. VII.10; 1987.V.15; 1991: VII.15-VII.16, VIII.31, IX.01; 1994: VIII.01-VIII.02; 2018.VIII.16.

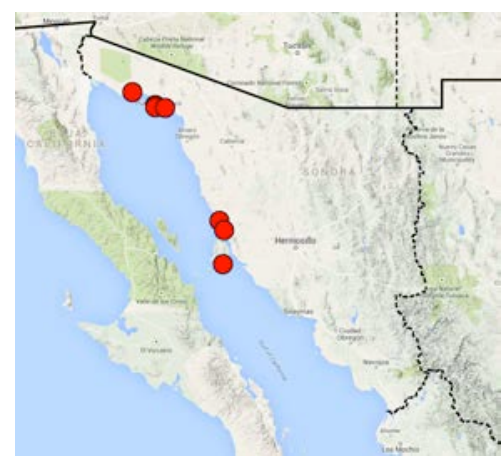

044. Microthylax sinaloae sinaloae (Bates, 1890)

Number examined: 94.

Municipalities: Empalme, Guaymas, Hermosillo.

Habitat: tidal flat

Vegetation: coastal saltscrub.

Elevation: $3 \mathrm{~m}$.

Collection dates: 1921.VII.08; 1952: VII.14, VII.18; 1960. VIII.10; 1966: VI.01-VI.02, VII.19, VII.21-VII.22; 1979: VII.07-VII.08.

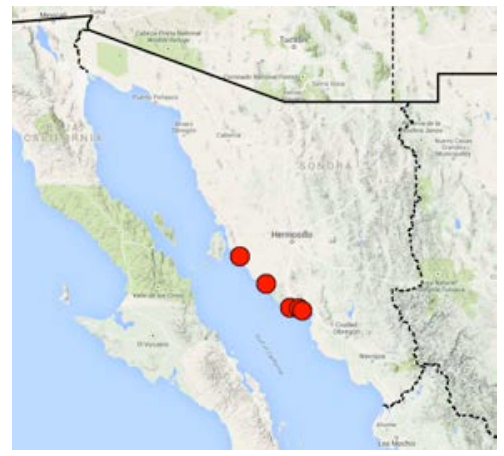

045. Opilidia macrocnema kino (Cazier, 1954)

Number examined: 147.

Municipality: Hermosillo.

Habitat: beach.

Vegetation: coastal dunescrub.

Elevation range: $5 \mathrm{~m}$.

Collection dates: 1952: VII.14, VII.18; 1958.X.24; 1966: VII.18-VII.19, VII.23; 1967.VI; 1970.VIII.24.

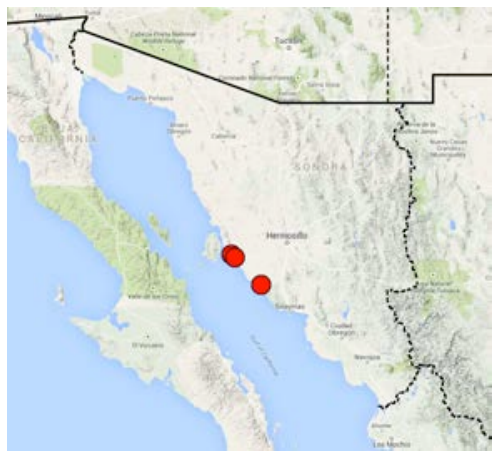

046. Opilidia macrocnema macrocnema (Chaudoir, 1852) Number examined: 130 .

Municipality: Empalme.

Habitat: ?

Vegetation: coastal thornscrub.

Elevation: ?

Collection dates: 1952.VIII.11; 1966: VI.01-VI.02, VII.21. 


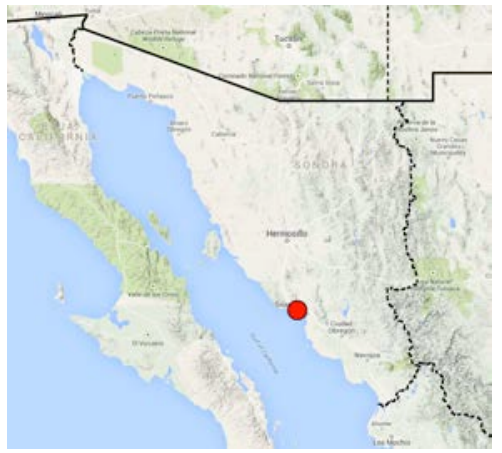

07. Omophronini

047. Omophron gilae LeConte, 1852

Number examined: 1.

Municipality: Pitiquito.

Habitat: ?

Vegetation: Sonoran desertscrub.

Elevation: ?

Collection date: 1952.VII.04.

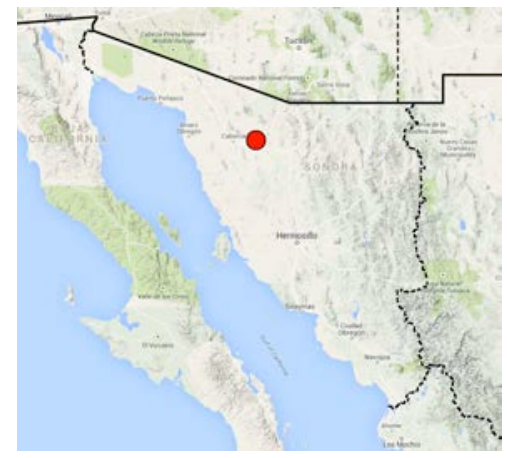

048. Omophron gratum Chaudoir, 1868

Number examined: 4.

Municipality: Cajeme.

Habitat: ?

Vegetation: coastal thornscrub.

Elevation: ?

Collection dates: 1950.VII.15; 1961.VI.11.

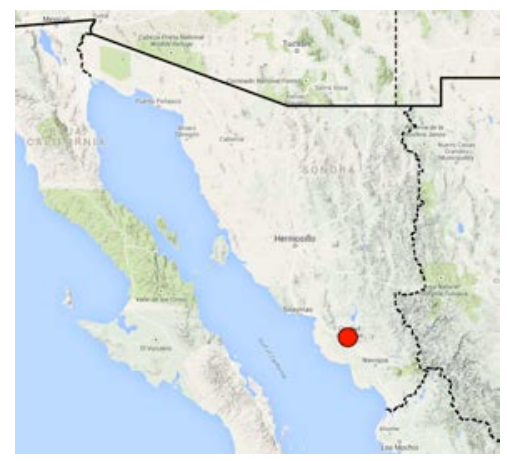

049. Omophron obliteratum G. Horn, 1870

Number examined: 13.

Municipalities: Hermosillo, Moctezuma, Puerto Peñasco,
Habitat: [at light]

Vegetation: foothills thornscrub; Sonoran desertscrub.

Elevation range: $944-2000 \mathrm{~m}$.

Collection dates: 1897.IV.19; 1976.IV.09; 1977.IV.09;

1983: VII.11-VII.12; 1985.VIII.01; 1993.VII.04.

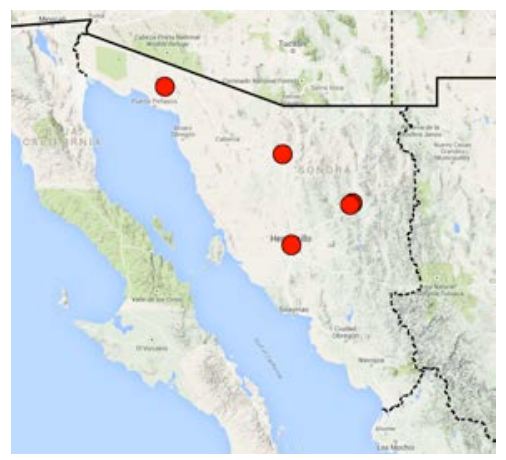

08. Pasimachini

050. Pasimachus californicus Chaudoir, 1850

Number examined: 22.

Municipalities: Agua Prieta, Álamos, Aconchi, Bacoachi, Cananea, Cucurpe, Fronteras, Huachinera, Nacozari de García, San Felipe de Jesús.

Habitat: cow pasture; rocky canyon, slope and mountainside.

Vegetation: Chihuahuan desertscrub; cottonwood-willow and sycamore riparian deciduous forest; desert grassland; foothills thornscrub; oak woodland; pine-oak forest; tropical deciduous forest.

Elevation range: $305-1860 \mathrm{~m}$.

Collection dates: 1964.VIII.21; 1982: VIII.05, VIII.19; 2012.VII.07; 2013: VII.02, VII.18; 2014: VIII.23, VIII.29; 2016.VII.02; 2017: I.09, VIII.13, VIII.16; 2019: VII.06, VII.22, VIII.04-VIII.06, VIII.16.

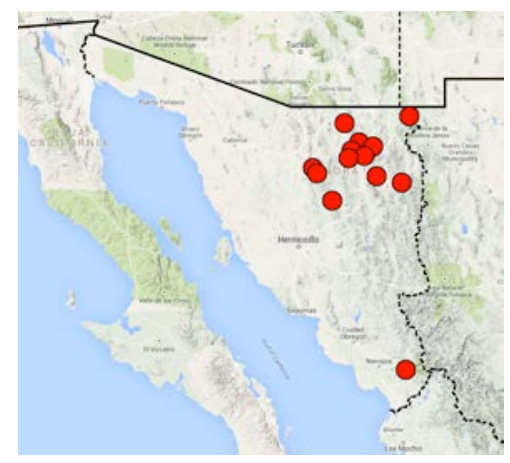

051. Pasimachus obsoletus LeConte, 1846

Number examined: 1 .

Municipality: Agua Prieta.

Habitat: ?

Vegetation: Chihuahuan desertscrub.

Elevation: ?

Collection date: 1965.VII.09.

Santa Ana. 


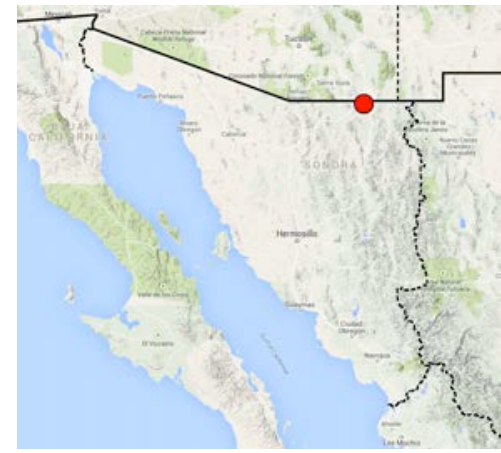

052. Pasimachus velutinus Van Dyke, 1943

Number examined: 8 .

Municipio: Álamos, Mazatán, Moctezuma, Onavas.

Habitat: [at light] (walked, did not fly)

Vegetation: foothills thornscrub; riparian tropical scrub; tropical deciduous forest.

Elevation range: 433 - $944 \mathrm{~m}$.

Collection dates: 1935: VII.07, VII.15; 1982.VIII.02; 1987: VII.26-VII.27, VII.30-VII.31; 2012.VI.26; 2019: VII.24, VIII.09.

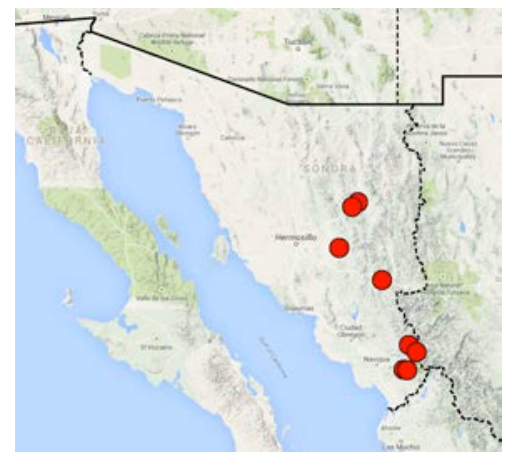

053. Pasimachus viridans LeConte, 1858

\section{Number examined: 96.}

Municipalities: Aconchi, Álamos, Bacanora, Cananea, Fronteras, Mazatán, Moctezuma, Nácori Chico, Nacozari de García, Ónavas, San Felipe de Jesús, Yécora.

Habitat: rocky arroyo, canyon, slope, and mountainside.

Vegetation: cypress-maple-alder-sycamore riparian forest; desert grassland; foothills thornscrub; oak woodland; pine-oak forest; sycamore riparian forest, oak woodland on slopes; tropical deciduous forest.

Elevation range: $1301-2160 \mathrm{~m}$.

Collection dates: 1935: VII.19-VII.20; 1980.IX.23 1982: VIII.06-VIII.07; 1983: VII.06-VII.07; 1987: VII.26VII.29; 1989: VII.11-VII.14; 1990: VI.28-VII.03; VIII.07-VIII.11; 1993.VII.08; 2012.VII.07; 2013: VII.02, VII.03; 2014: VII.28, VIII.02, VIII.25, IX.20; 2016: VIII.15-VIII.16, VIII.26; 2017: VIII.12-VIII.15; 2018: VI.27, VI.29, VIII.05-VIII.09; 2019: VIII.05VIII.06.

Variation: Only 6 of the 96 specimens have reddish pronotal and elytral margins rather than the typical green margins (see frontispiece).

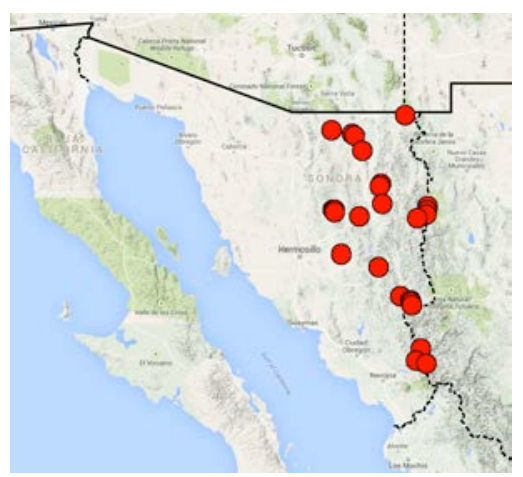

09. Scaritini

054. Scarites subterraneus Fabricius, 1775

Number examined: 7.

Municipalities: Agua Prieta, Hermosillo, Ímuris, Moctezuma, Navojoa.

Habitat: ?

Vegetation: Chihuahuan and Sonoran desertscrub; riparian tropical scrub; coastal and foothills thornscrub.

Elevation range: $15-1350 \mathrm{~m}$.

Collection dates: 1908.IX.01; 1982: VIII.01, VIII.20, VIII.24; 1987.XI.02.

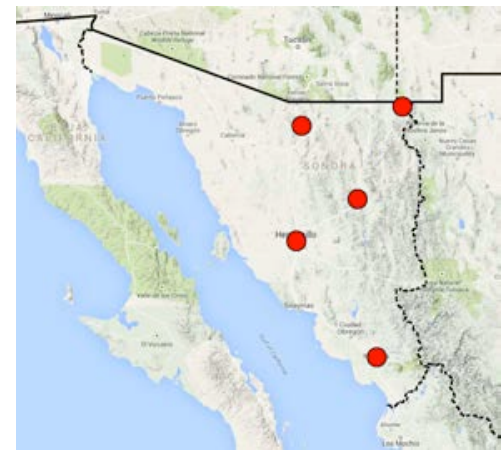

\section{Clivinini 055. Ardistomis sp.}

Number examined: 1 .

Municipality: Ímuris

Habitat: ?

Vegetation: cottonwood-willow riparian forest. Elevation: $1040 \mathrm{~m}$.

Collection date: 1982.VIII.24.

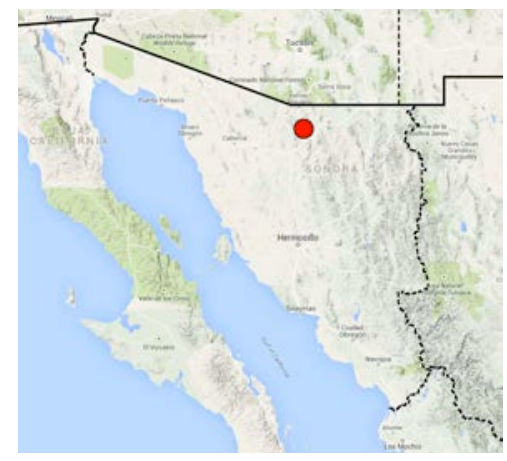


056. Aspidoglossa subangulata (Chaudoir, 1843)

Number examined: 3 .

Municipalities: Carbó, Hermosillo, Navojoa.

Habitat: ?

Vegetation: coastal thornscrub; Sonoran desertscrub.

Elevation:?

Collection dates: 1953: VII.09-VII.16, IX.26; 1960. VIII.08.

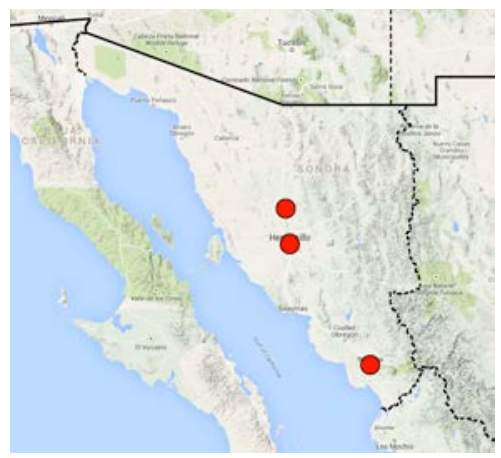

\section{Aspidoglossa sp.}

Number examined: 21 .

Municipalities: Aconchi, Álamos, Moctezuma, Nácori Chico, Rayón, Santa Ana, Yécora.

Habitat: urban. [at light]

Vegetation: foothills thornscrub; oak woodland; Sonoran desertscrub; tropical deciduous forest.

Elevation range: $542-1752 \mathrm{~m}$.

Collection dates: 1982: VIII.09, VIII.23; 1983: VII.03, VII.11-VII.12; 1989: VI.11-VI.13; 1990: VII.02-VII.03; 1993.VII.09; 2014:VIII.29; 2019.VIII.15.

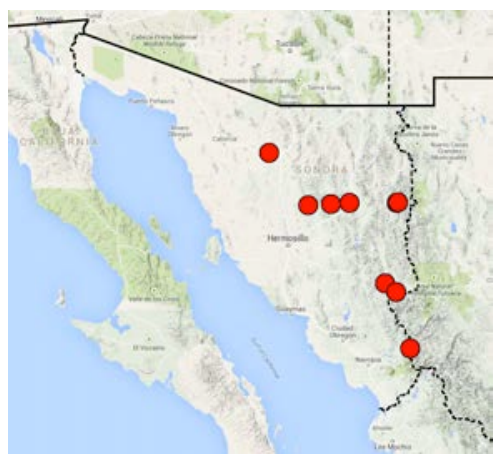

058. Clivina lucida Putzeys, 1866

Number examined: 1 .

Municipality: Navojoa.

Habitat: ?

Vegetation: coastal thornscrub.

Elevation:?

Collection date: 1956.VI.24.

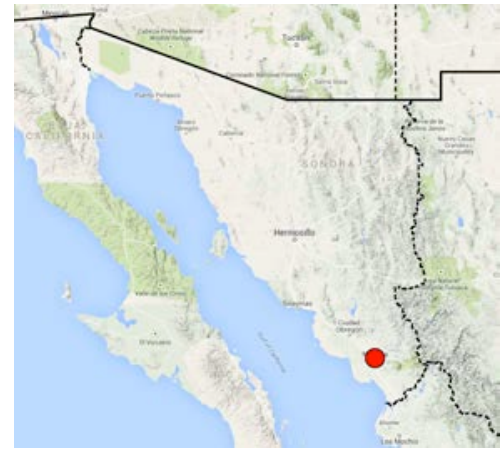

059. Clivina sp. 1

Number examined: 6 .

Municipality: Carbó.

Habitat: ?

Vegetation: Sonoran desertscrub.

Elevation:?

Collection date: 1965.VII.31.

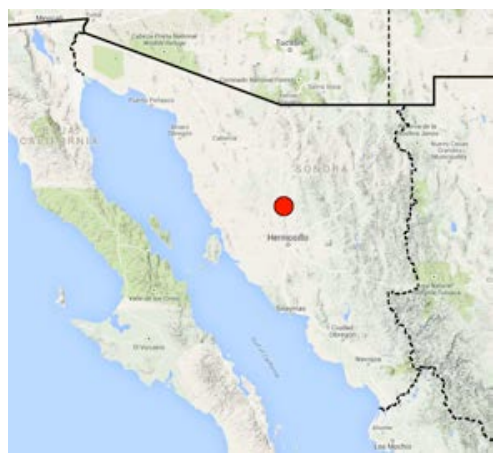

060. Clivina spp. (may be more than one species)

Number examined: 87.

Municipalities: Agua Prieta, Álamos, Granados, Hermosillo, Ímuris, Moctezuma, Nácori Chico, Nacozari de García, Navojoa, Santa Ana, Ures.

Habitat: rocky canyon; urban. [at light]

Vegetation: desert grassland; foothills thornscrub; oak woodland; riparian tropical forest; Sonoran desertscrub; sycamore riparian deciduous forest; tropical deciduous forest.

Elevation range: $235-1450 \mathrm{~m}$.

Collection dates: 1954.VIII.22; 1969.VIII.12; 1982: VIII.01, VIII.11, VIII.19-VIII.20, VIII.23, VIII.24; 1983: VII.03-VII.04, VII.11-VII.12; 1987: VII.30VII.31; 1993.VII.05; 2012.IX.02; 2017: VII.21-VII.23. 


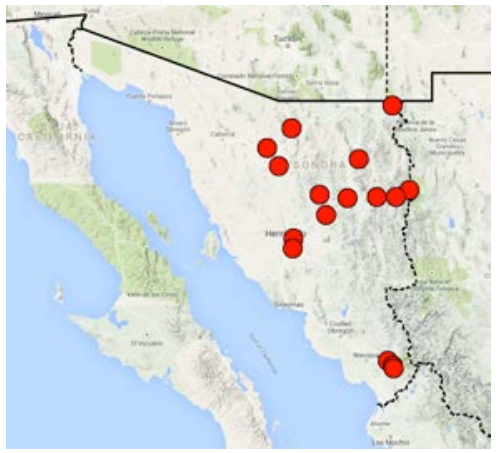

061. Schizogenius auripennis Bates, 1881

Number examined: 70.

Municipios: Agua Prieta, Álamos, Hermosillo, Moctezuma.

Habitat: [at light]

Vegetation: Chihuahuan and Sonoran deseretscrub; tropical deciduous forest.

Elevation: $1350 \mathrm{~m}$.

Collection dates: 1953: VII.09-VII.16; 1960.VIII.12; 1969.VIII.12; 1982: VIII.19-VIII.20.

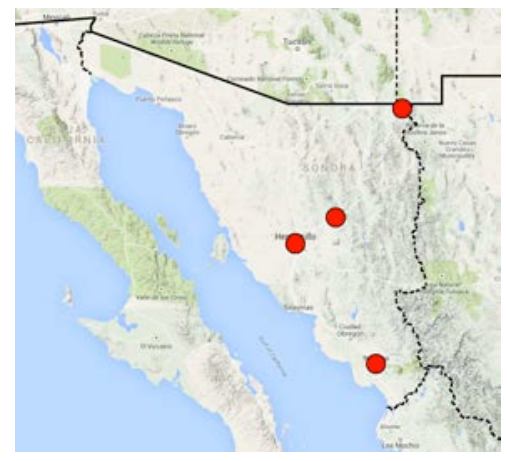

062. Schizogenius falli Whitehead, 1972

Number examined: 122.

Municipalities: Agua Prieta, Álamos, Carbó, Guaymas, Hermosillo, Moctezuma, Ónavas, Santa Ana.

Habitat: [at light]

Vegetation: Chihuahuan and Sonoran desertscrub; foothills thornscrub; oak woodland; tropical deciduous forest.

Elevation range: $710-1350 \mathrm{~m}$.

Collection dates: 1935.VI.27; 1953: VII.09-VII.16; 1960:

VIII.08, VIII.10, VIII.12; 1969.VIII.12; 1982: VIII.19-

VIII.20, VIII.23; 1983: VII.11-VII.12; 2019.VII.22.

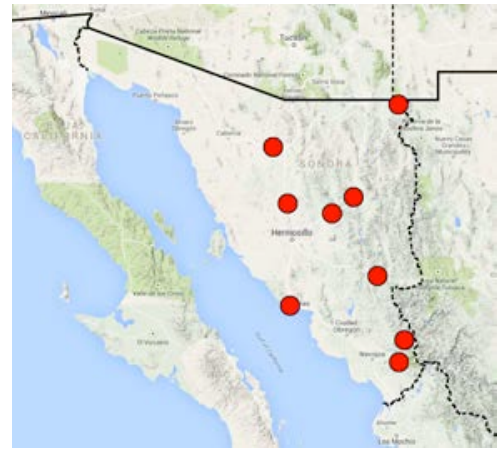

063. Schizogenius longipennis Putzeys, 1866

Number examined: 143.

Municipalities: Álamos, Moctezuma, Nácori Chico, Ónavas.

Habitat: rocky stream margin. [at light]

Vegetation: cypress-maple-alder-sycamore riparian forest; foothills thornscrub; oak woodland; pine-oak forest; riparian tropical scrub; tropical deciduous forest.

Elevation range: $917-1648 \mathrm{~m}$.

Collection dates: 1960.VIII.12. 1982: VIII.01, VIII.06VIII.07, VIII.09; 1983: VII.03, VII.11-VII.12; 1987: VII.26-VII.27; 1989.VII.08; 1993.VII.04; 2018: VIII.05-VIII.06.

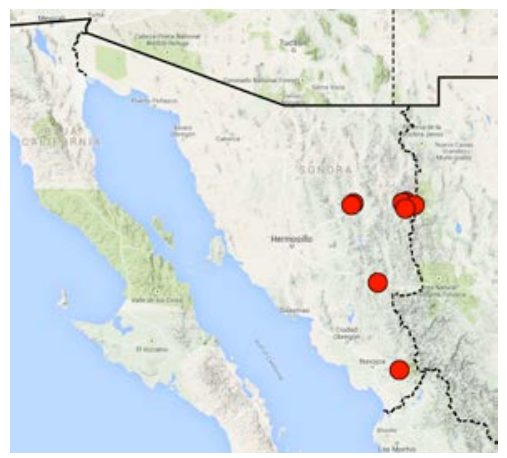

064. Schizogenius neovalidus Whitehead, 1972

Number examined: 5

Municipality: Agua Prieta.

Habitat: [at light]

Vegetation: Chihuahuan desertscrub.

Elevation: $1350 \mathrm{~m}$.

Collection dates: 1982: VIII.19-VIII.20.

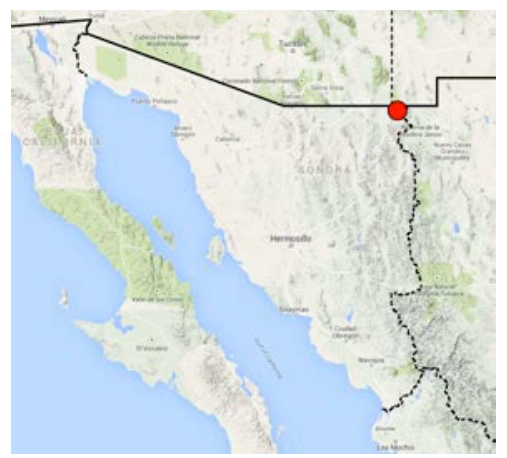


065. Schizogenius pluripunctatus LeConte, 1852

Number examined: 7.

Municipalities: Agua Prieta, Álamos, Nácori Chico.

Habitat: rocky stream margin. [at light]

Vegetation: Chihuahuan desertscrub; pine-oak forest.

Elevation range: $945-1350 \mathrm{~m}$.

Collection dates: 1982: VIII.19 VIII.20; 1983. VII.03; 2018.VIII.05; 2019.VII.23.

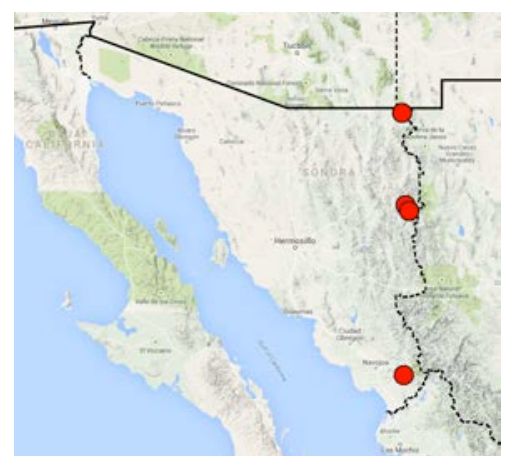

066. Schizogenius pygmaeus Van Dyke, 1925

Number examined: 102.

Municipios: Agua Prieta, Álamos, Hermosillo, Guaymas, Ímuris, Moctezuma, Nácori Chico, Ónavas, Santa Ana.

Habitat: [at light]

Vegetation: Chihuhuan and Sonoran desertscrub; cottonwood gallery forest; cypress-maple-alder-sycamore riparian forest; foothills thornscrub; oak woodland; pineoak forest; oak forest; pine-oak forest; riparian tropical scrub; tropical deciduous forest.

Elevation range: $710-1690 \mathrm{~m}$.

Collection dates: 1953: VII.09-VII.16; 1960: VIII.10, VIII.12; 1969.VIII.12; 1982: VIII.01, VIII.19-VIII.20, VIII.23; 1983: VII.03, VII.11-VII.12; 1986.VIII.30; 1987: VII.26-VII.27; 1989: VII.11-VII.13; 1990. VIII.06; 2018.VIII.06; 2019.VII.22.

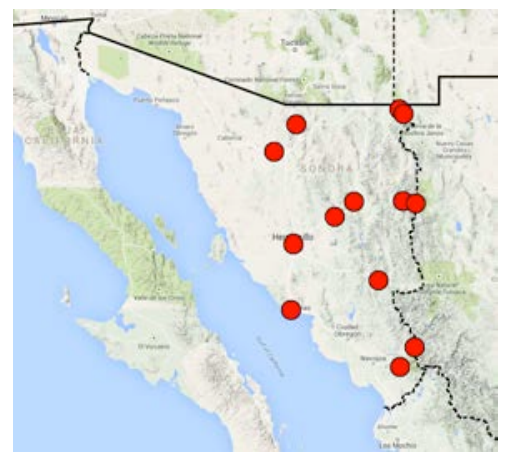

067. Semiardistomis propinquus (Putzeys, 1866)

Number examined: 35

Municipality: Yécora

Habitat: [at light]

Vegetation: pine-oak forest.

Elevation range: $1547-1752 \mathrm{~m}$.

Collection dates: 1990: VI.30-VII.03.

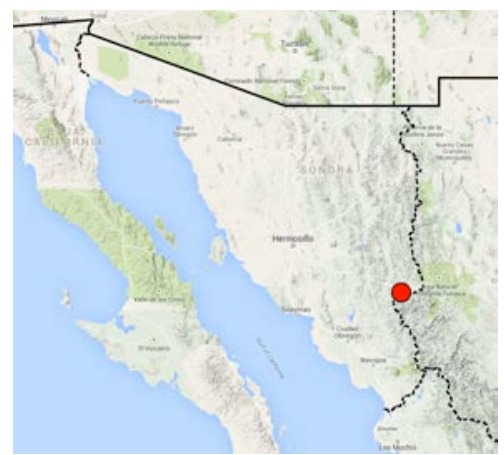

\section{Dyschiriini}

068. Dyschirius analis LeConte, 1852

Number examined: 19.

Municipalities: Álamos, Guaymas, Hermosillo.

Habitat: [at light]

Vegetation: Sonoran desertscrub; tropical deciduous forest.

Elevation:?

Collection dates: 1953: VII.09-VII.16; 1960: VIII.10, VIII.12; 1965.VIII.01.

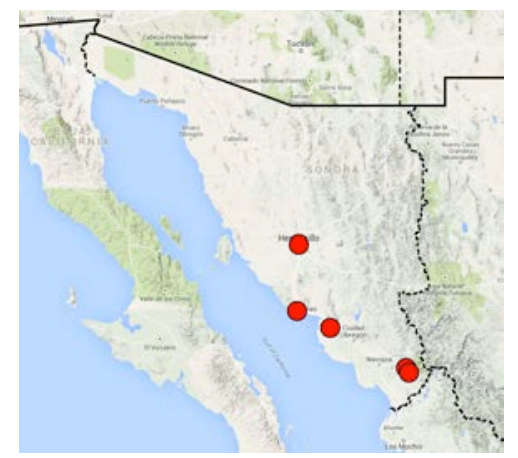

069. Dyschirius aratus LeConte, 1852

Number examined: 1.

Municipality: Hermosillo.

Habitat: ?

Vegetation: Sonoran desertscrub.

Elevation: ?

Collection date: 1953: VII.09-VII.16.

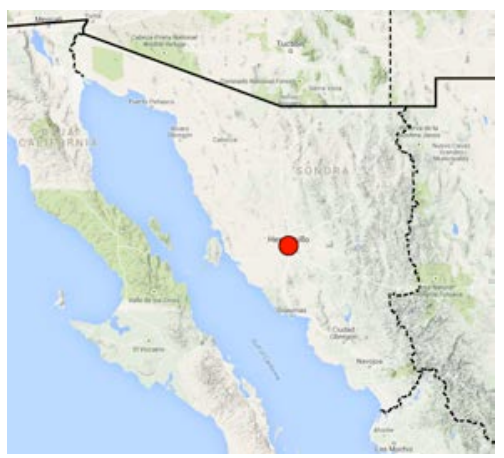


070. Dyschirius politus (Dejean, 1825)

Number examined: 5 .

Municipality: Hermosillo.

Habitat: ?

Vegetation: Sonoran desertscrub.

Elevation: ?

Collection dates: 1953: VII.09-VII.16.

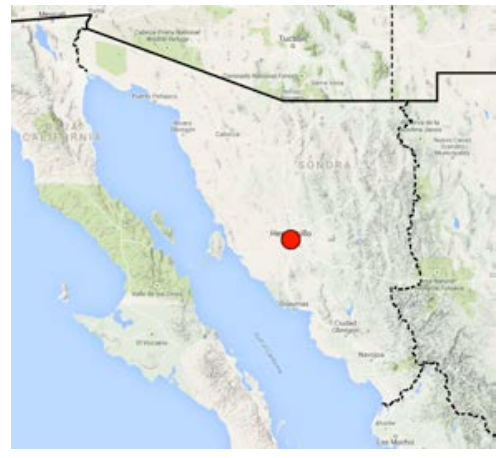

071. Dyschirius terminatus LeConte, 1848

Number examined: 7.

Municipalities: Álamos, Hermosillo

\section{Habitat: ?}

Vegetation: Sonoran desertscrub; tropical deciduous forest. Elevation: ?

Collection dates: 1953: VII.09-VII.16; 1960.VIII.12.

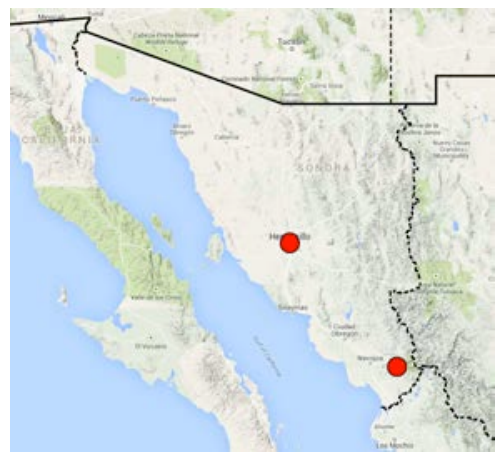

\section{Dyschirius sp.}

Number examined: 9 .

Municipalities: Agua Prieta, Ímuris, Moctezuma.

Habitat: [at light] [treading]

Vegetation: Chihuahuan and desertscrub; foothills thornscrub.

Elevation range: $944-1350 \mathrm{~m}$.

Collection dates: 1982: VIII.19-VIII.20; 1983: VII.11VII.12; 1986.VIII.30; 1993.VII.04.

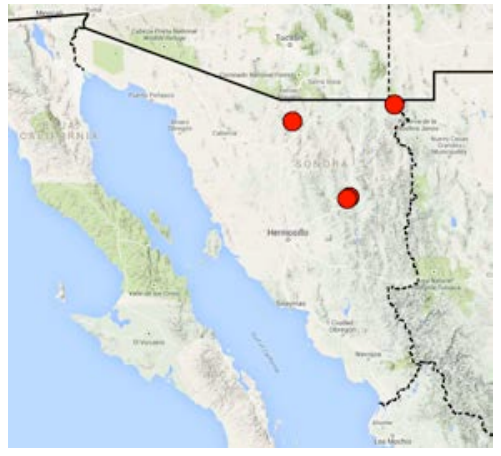

\section{Trechini \\ 073. Cnides sp. 2}

Number examined: 3 .

Municipality: Álamos.

Habitat: riparian.

Vegetation: tropical deciduous forest.

Elevation: ?

Collection dates: 1975.VIII.11; 1999.VII.20.

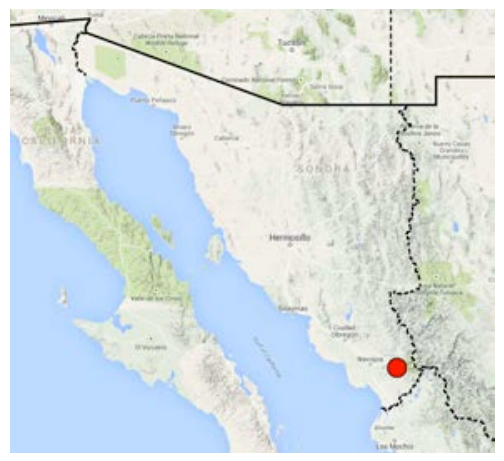

\section{Bembidiini}

074. Bembidion (Cyclolopha) poculare Bates, 1884

Number examined: 1 .

Municipality: Huachinera.

Habitat: ?

Vegetation: desert grassland.

Elevation: $1150 \mathrm{~m}$.

Collection date: 1982.VIII.05.

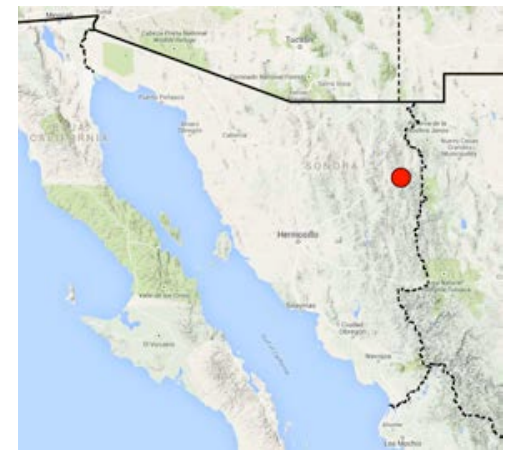


075. Bembidion (Eupetedromus) aratum (LeConte, 1852)

Number examined: 1.

Municipality: Ímuris.

Habitat: cottonwood-willow riparian forest.

Vegetation: ?

Elevation: $1040 \mathrm{~m}$.

Collection date: 1983.VIII.24.

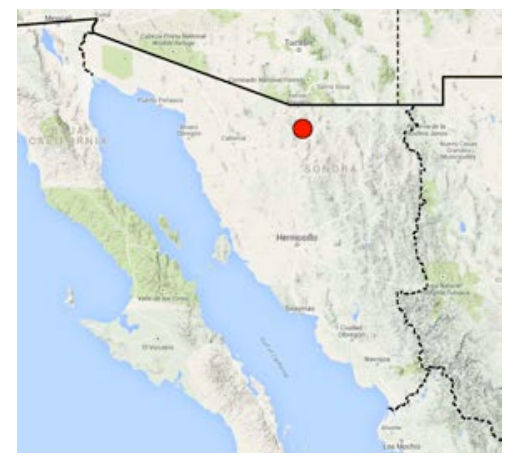

076. Bembidion (Furcacampa) egens Casey, 1918 Number examined: 29.

Municipalities: Guaymas, Hermosillo, Ímuris.

Habitat: ?

Vegetation: Sonoran desertscrub.

Elevation: ?

Collection dates: 1956.VI.24; 1965: VII.31, VIII.11.

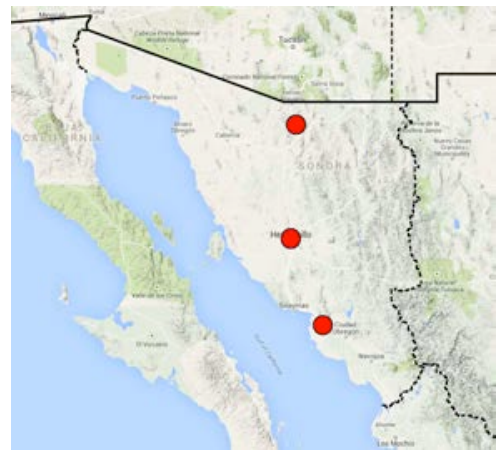

077. Bembidion (Furcacampa) sp. cf. cognatum Dejean, 1831

Number examined: 8 .

Municipality: Hermosillo.

Habitat: ?

Vegetation: Sonoran desertscrub.

Elevation: ?

Collection dates: 1953: VII.09-VII.16.

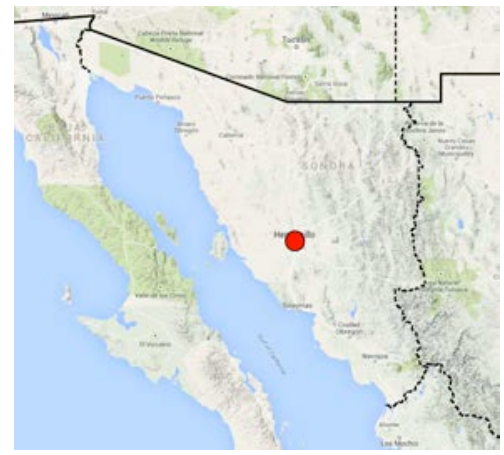

078. Bembidion (Furcacampa) sp. cf. nogalesium Casey, 1924

Number examined: 2 .

Municipality: Guaymas.

Habitat: ?

Vegetation: Sonoran desertscrub.

Elevation: ?

Collection date: 1923.IX.23.

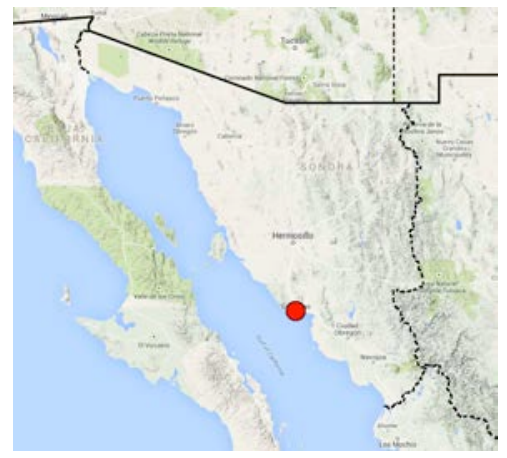

079. Bembidion (Furcacampa) sp. 1

Number examined: 1 .

Municipality: Álamos.

Habitat: ?

Vegetation: tropical deciduous forest.

Elevation: ?

Collection date: 1961.VI.13.

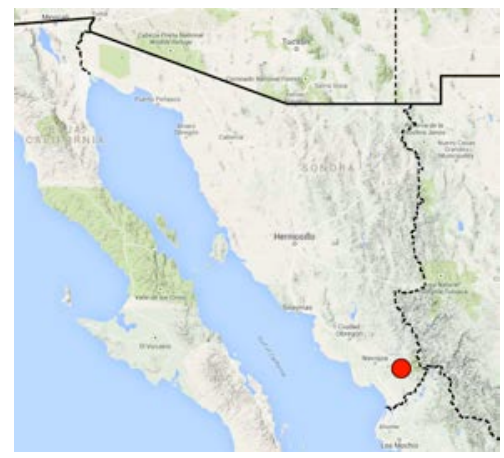


080. Bembidion (Furcacampa) sp. 2

Number examined: 3 .

Municipality: Hermosillo.

Habitat: ?

Vegetation: Sonoran desertscrub.

Elevation: ?

Collection dates: 1953: VII.09-VII.16.

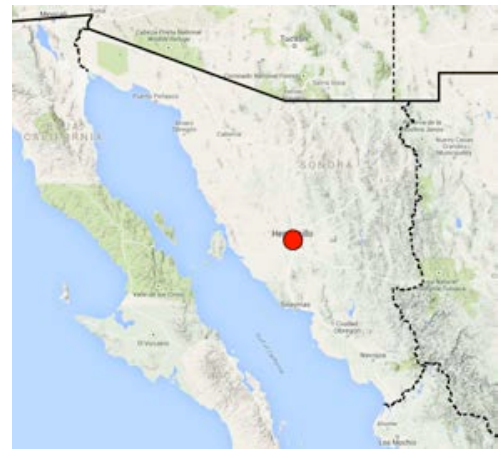

081. Bembidion (Notaphus) rapidum species group sp. \#1

Number examined: 1.

Municipality: Agua Prieta.

Habitat:?

Vegetation: oak woodland; riparian vegetation.

Elevation: $1291 \mathrm{~m}$.

Collection date: 2017.IV.23.

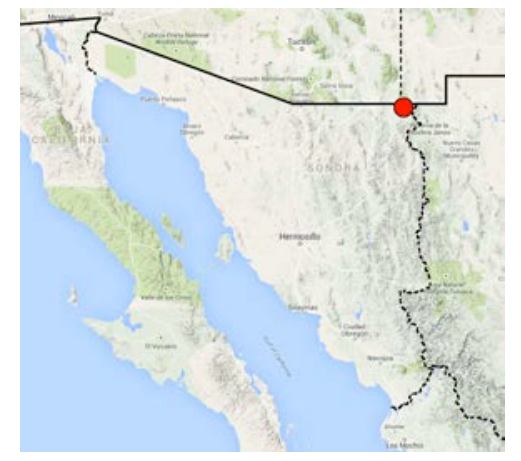

082. Bembidion (Notaphus) rapidum species group sp. \#2

Number examined: 1.

Municipality: Agua Prieta.

Habitat: ?

Vegetation: oak woodland; riparian vegetation.

Elevation: $1291 \mathrm{~m}$.

Collection date: 2017.IV.23.

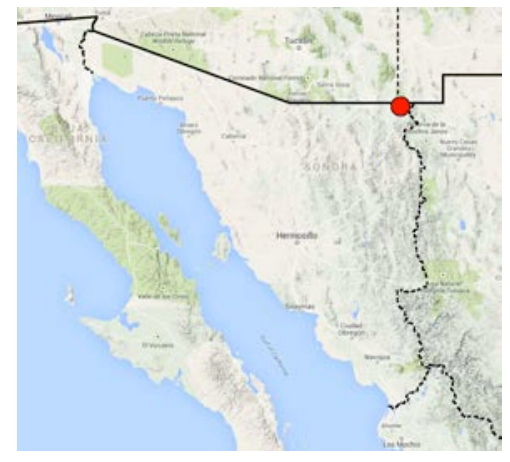

083. Bembidion (Notaphus) rapidum species group sp. \#3 Number examined: 15.

Municipalities: Empalme, Guaymas, Hermosillo, Navojoa.

Habitat: ?

Vegetation: coastal thornscrub; Sonoran desertscrub Elevation:?

Collection dates: 1923.IX.23; 1953: VII.9-VII.16; 1954 : VIII.22, VIII.24.

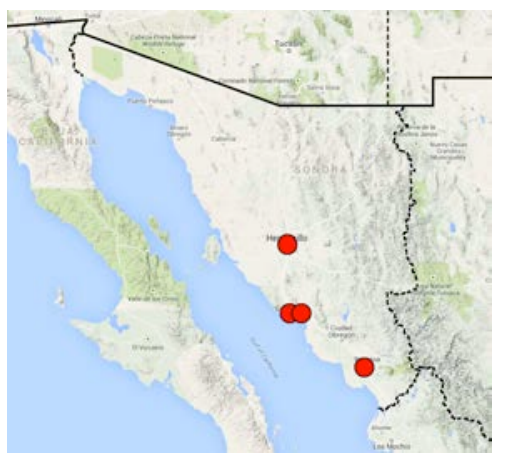

084. Bembidion (Notaphus) semifasciatum species group sp. \#1

Number examined: 32 .

Municipality: Ímuris.

Habitat: bog; marsh.

Vegetation: cottonwood-willow riparian forest.

Elevation: 914 - $1040 \mathrm{~m}$.

Collection dates: 1965.VII.31; 1967.VII.27; 1982.VIII.24; 1986. VIII.30.

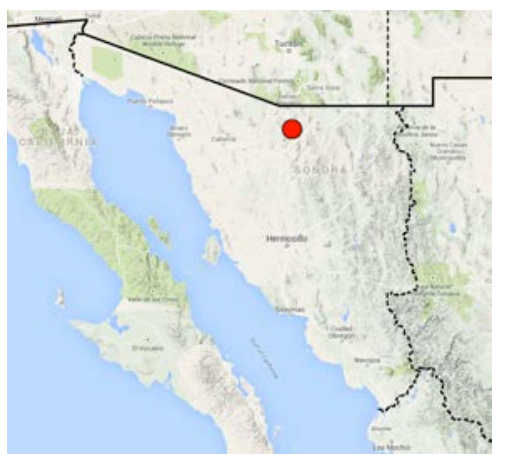


Number examined: 1 .

Municipality: Agua Prieta.

Habitat: [at light]

Vegetation: Chihuahuan desertscrub.

Elevation: ?

Collection date: 2017.IV.23.

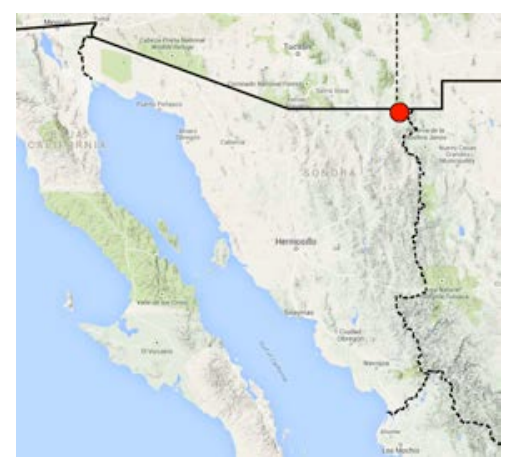

086. Bembidion (Ochthedromus) cheyennense Casey, 1918

Number examined: 1 .

Municipality: Ímuris.

Habitat: ?

Vegetation: cottonwood-willow riparian forest.

Elevation: ?

Collection date: 1965 .VII.31.

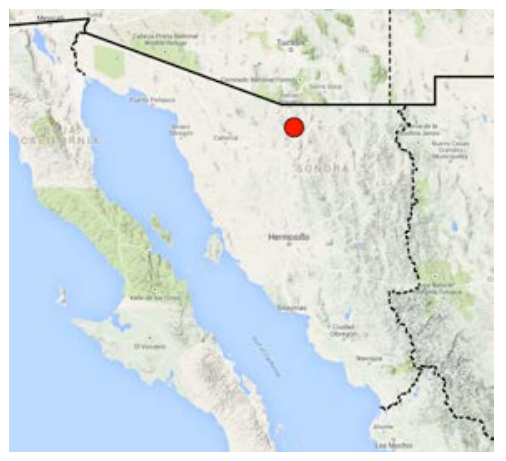

087. Bembidion (Peryphus) lugubre LeConte, 1857

Number examined: 2 .

Municipality: Agua Prieta.

Habitat: ?

Vegetation: Riparian vegetation, oak woodland.

Elevation: $1291 \mathrm{~m}$.

Collection date: 2017.IV.23.

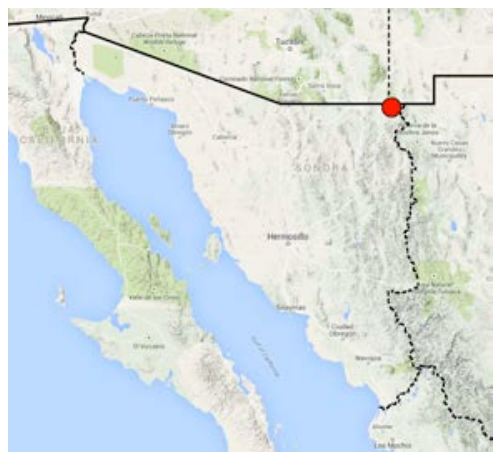

088. Bembidion (Peryphus) mexicanum Dejean, 1831

Number examined: 30 .

Municipalities: Nácori Chico, Santa Cruz.

Habitat: rocky stream margin.

Vegetation: cypress-maple-alder-sycamore riparian forest; oak woodland; pine-oak forest.

Elevation range: $1549-1648 \mathrm{~m}$.

Collection dates: 2018: VIII.05-VIII.08; 2019.IV.25.

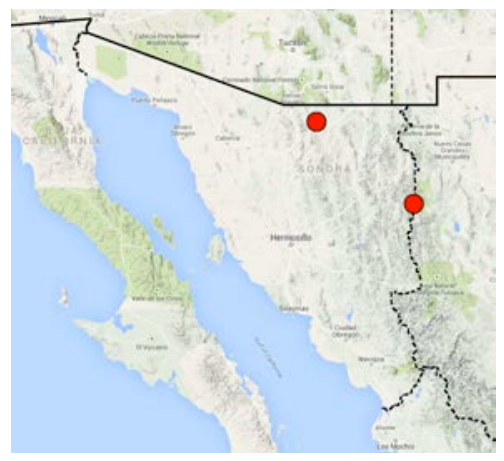

089. Bembidion (Peryphus) perspicuum (LeConte, 1848)

Number examined: 11.

Municipality: Agua Prieta.

Habitat: [at light]

Vegetation: oak woodland; riparian vegetation.

Elevation: $1291 \mathrm{~m}$.

Collection date: 2017.IV.23.

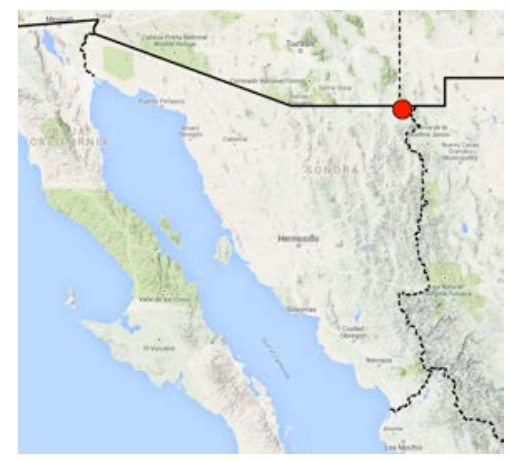




\section{Bembidion (Peryphus) mexicanum}

species group sp. son-1

Number examined: 11.

Municipalities: Cananea, Nacozari de García, Nogales.

Habitat: rocky arroyo, stream margin and canyon.

Vegetation: oak woodland; pine-oak forest; riparian deciduous forest;

Elevation range: $1161-1928 \mathrm{~m}$.

Collection dates: 2014.VIII.24; 2015: VII.12, VIII.12.

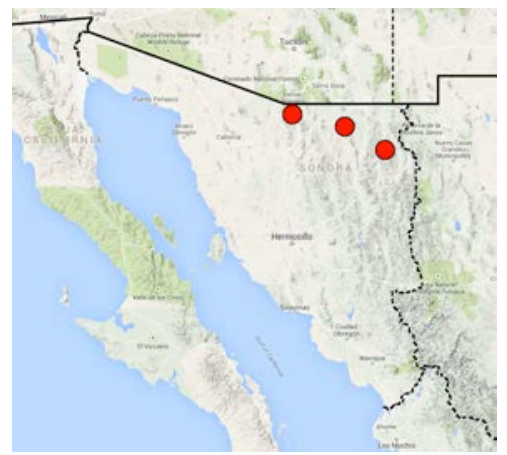

091. Bembidion (Peryphus) mexicanum species group sp. son-2

Number examined: 7.

Municipalities: Fronteras, Nacozari de García.

Habitat: cow pasture; rocky arroyo, stream margin.

Vegetation: oak woodland; pine-oak forest.

Elevation: 1429 - 1928 m.

Collection dates: 2015.VIII.12; 2016.VII.02.

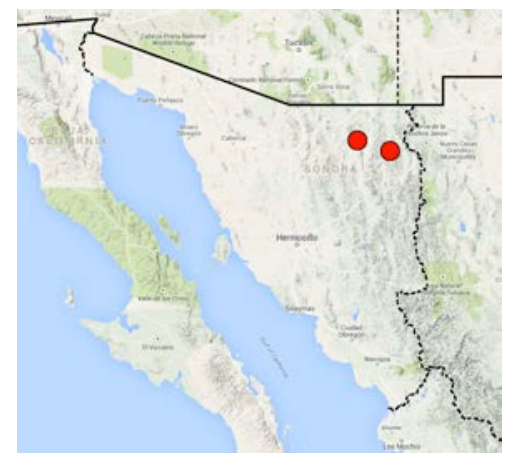

092. Bembidion (Peryphus) mexicanum species group sp. B2

Number examined: 5 .

Municipality: Agua Prieta.

Habitat:?

Vegetation: oak woodland; sycamore riparian forest.

Elevation: 1350 - $1690 \mathrm{~m}$.

Collection dates: 1982: VIII.19-VIII.20.

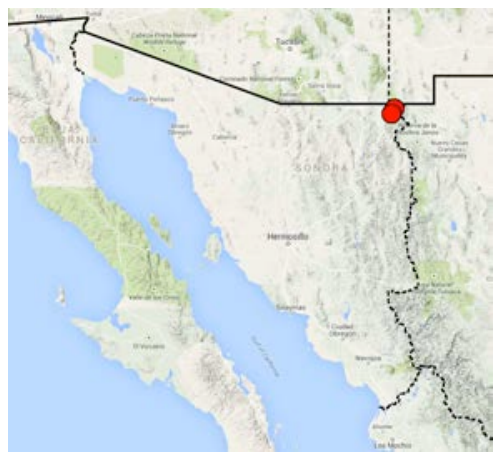

093. Bembidion (Peryphus) mexicanum species group sp. B3

Number examined: 2 .

Municipality: Agua Prieta.

Habitat: ?

Vegetation: oak woodland; sycamore riparian forest.

Elevation: 1429 - $1928 \mathrm{~m}$.

Collection date: 1982.VIII.19.

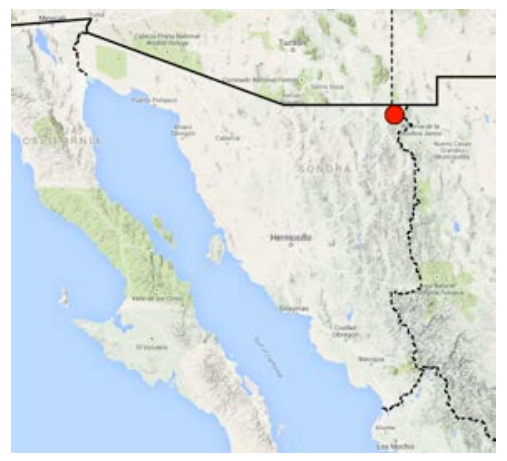

094. Bembidion (Peryphus) mexicanum species group sp. B4

Number examined: 21.

Municipalities: Agua Prieta, Ímuris, Moctezuma, Nácori Chico, Yécora.

Habitat: arroyo, stream margin. [at light]

Vegetation: foothills thornscrub; oak woodland; pine-oak forest; sycamore riparian forest.

Elevation: 914 - $1950 \mathrm{~m}$.

Collection dates: 1967.VII.27; 1982: VIII.06-VII.07, VIII.19-VIII.20; 1983: VII.03, VII.11-VII.12; 1990. VI.30.

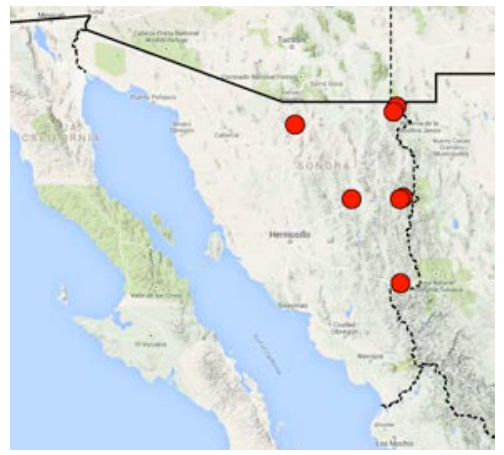


095. Bembidion (Peryphus) mexicanum species group sp.

\section{B5}

Number examined: 1 .

Municipality: Huachinera.

Habitat: ?

Vegetation: Pine-oak forest.

Elevation: $2090 \mathrm{~m}$.

Collection date: 1982: VIII.03-VIII.04.

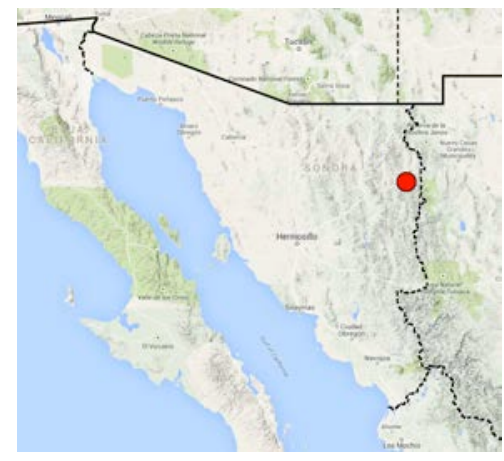

096. Elaphropus conjugens (Notman, 1918)

Number examined: 1.

Municipality: Yécora.

Habitat: ?

Vegetation: pine-oak forest.

Elevation: ?

Collection date: 1990: VIII.08-VIII.11.

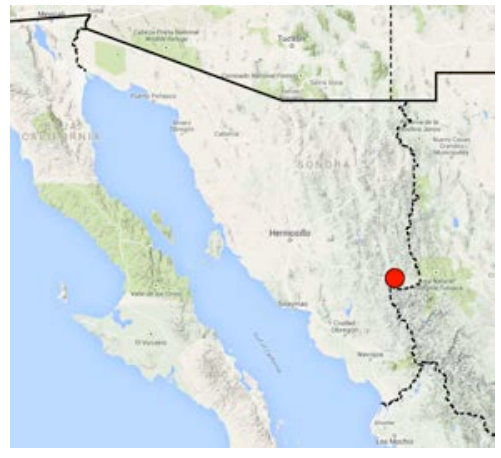

097. Elaphropus dolosus (LeConte, 1848)

Number examined: 37.

Municipality: Hermosillo.

Habitat: ?

Vegetation: Sonoran desertscrub.

Elevation: ?

Collection dates: 1953: VII.09-VII.16.

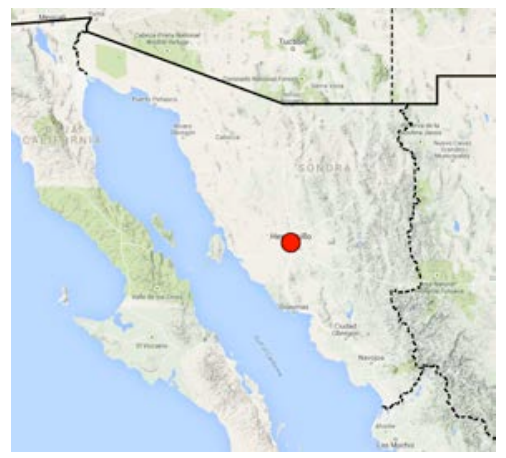

098. Elaphropus mellitus (Casey, 1918)

Number examined: 122.

Municipalities: Álamos, Guaymas, Hermosillo, San Javier.

Habitat: ?

Vegetation: coastal thornscrub; Sonoran desertscrub; tropical deciduous forest.

Elevation: ?

Collection dates: 1897.IV.19; 1929.IV.03; 1953: VII.09VII.16; 1960: VIII.10, VIII.12; 1963.II.27.

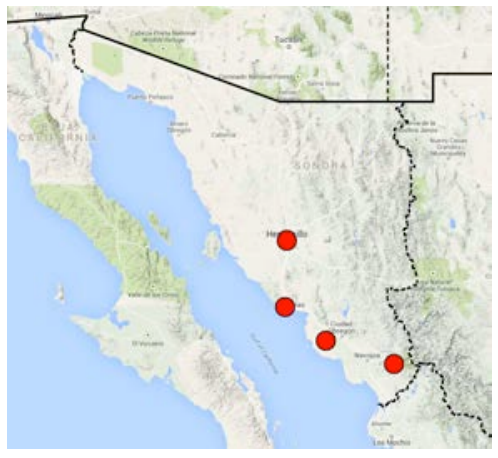

099. Elaphropus obesulus (LeConte, 1852)

Number examined: 17.

Municipalities: Hermosillo, Saric.

Habitat: ?

Vegetation: Sonoran desertscrub.

Elevation: ?

Collection dates: 1929.VI.02; 1953: VII.09-VII.16.

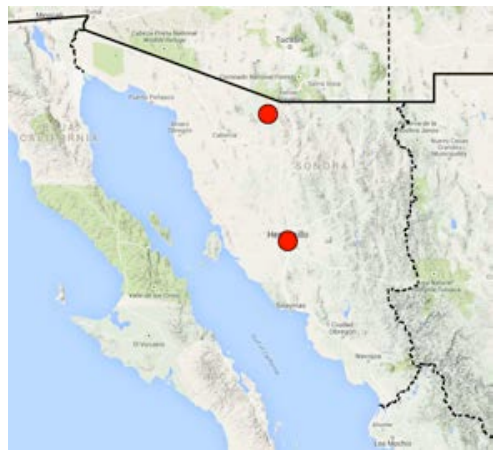


100. Elaphropus sp. CASC 37-B-MEX

Number examined: 3 .

Municipality: Álamos.

Habitat: ?

Vegetation: tropical deciduous forest.

Elevation:?

Collection date: 1960.VIII.12.

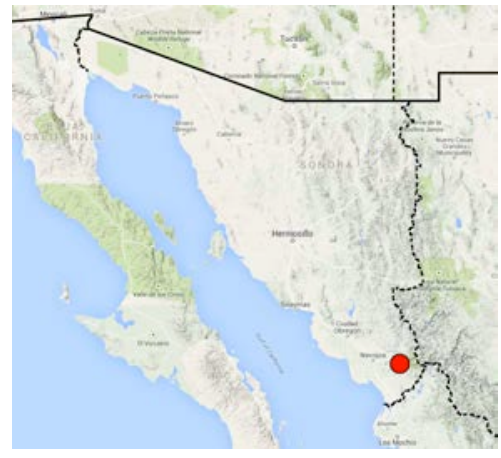

101. Elaphropus sp. CASC 3-B-US

Number examined: 13.

Municipality: Álamos.

Habitat: ?

Vegetation: tropical deciduous forest.

Elevation: ?

Collection date: 1960.VIII.12.

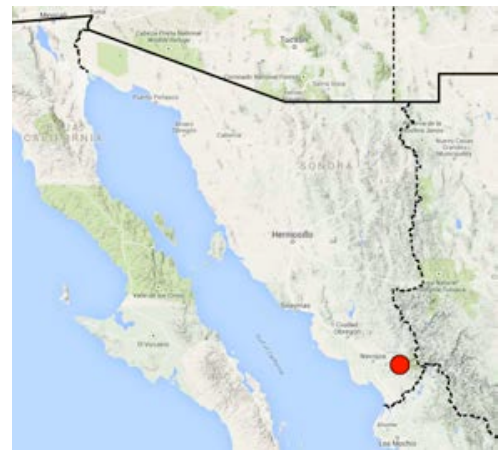

102. Elaphropus sp. 1

Number examined: 8 .

Municipality: Ónavas.

Habitat: [at light]

Vegetation: foothills thornscrub; oak woodland.

Elevation: $917 \mathrm{~m}$.

Collection dates: 1987: VII.26-VII.27.

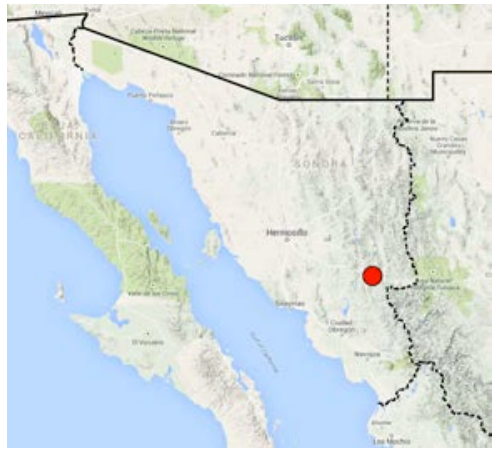

103. Elaphropus spp. (may be more than one species)

Number examined: 289.

Municipalities: Agua Prieta, Álamos, Altar, Carbo, Fronteras, Huachinera, Ímuris, Moctezuma, Nácori Chico, Nacozari de García, Nogales, Ónavas, Santa Ana, Yécora.

Habitat: cow pasture; rocky arroyo, stream margin, and mountainside; rocky; flood litter. [at light]

Vegetation: cypress-maple-alder-sycamore riparian forest; cottonwood-willow riparian forest; foothills thornscrub; oak woodland; pine-oak forest; riparian tropical scrub; Sonoran desertscrub; tropical deciduous forest.

Elevation range: $710-2278 \mathrm{~m}$.

Collection dates: 1941.V.24; 1942.II.11; 1952: IX.19IX.20; 1953: VII.09-VII.16; 1959.VIII.14; 1960. VIII.12; 1961: VI.12-VI.13; 1962: IV.16, V.22; 1967. VIII.20; 1970.VI.18; 1972.X.25; 1980: IX.17-IX.18, IX.23, IX.26-IX.27; 1982: VIII.01, VIII.03-VIII.04, VIII.19-VIII.20, VIII.23-VIII.24; 1983: VII.03-VII.05, VII.11-VII.12; 1986.VIII.30; 1989: VII.11-VII.13; 1990: VI.30-VII.01, VIII.06, VIII.07-VIII.11; 1993: VII.04, VII.05; 2015.VIII.11; 2016.VII.02; 2017. VIII.15; 2018: III.19, VIII.05-VIII-06, VIII.08.

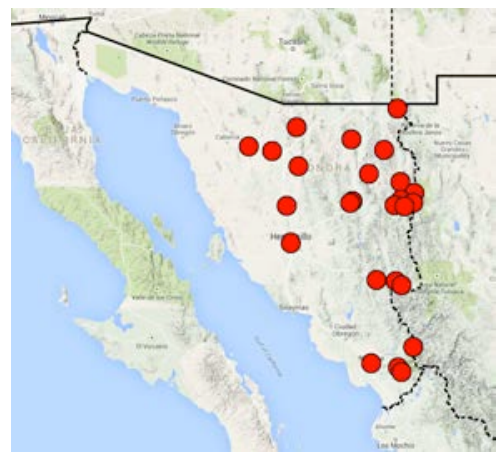

104. Micratopus sp.

Number examined: 3 .

Municipalities: Empalme, Guaymas, Hermosillo.

Habitat: ?

Vegetation: coastal thornscrub; Sonoran desertscrub. Elevation:?

Collection dates: 1954.VIII.24; 1962.XI.15; 1973.V. 


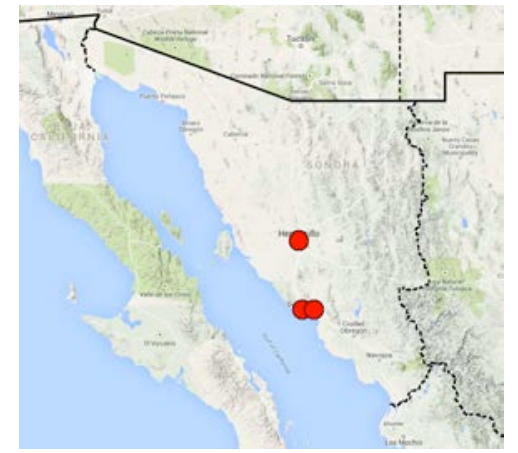

105. Paratachys sp. USNM \#29

Number examined: 2 .

Municipality: Álamos.

Habitat: ?

Vegetation: tropical deciduous forest.

Elevation:?

Collection date: 1960.VIII.12.

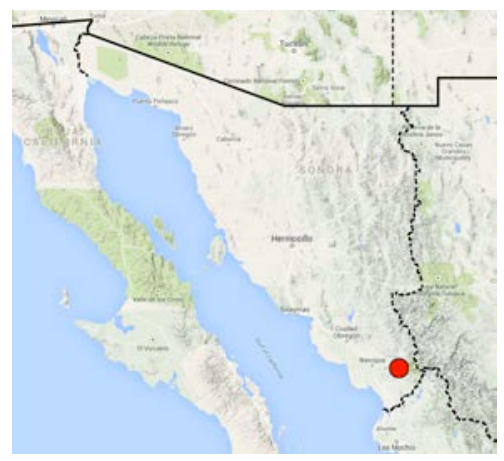

106. Paratachys sp. USNM \#60

Number examined: 4.

Municipalities: Álamos, Hermosillo.

Habitat: ?

Vegetation: Sonoran desertscrub; tropical deciduous forest.

Elevation: ?

Collection dates: 1953: VII.09-0VII.16; 1960.VIII.12.

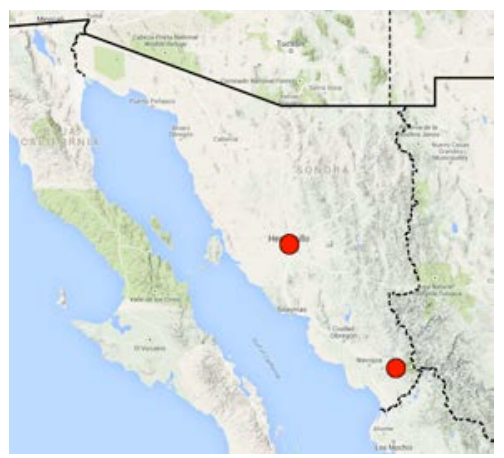

107. Paratachys sp. USNM \#105

Number examined: 2.

Municipalities: Álamos, San Javier.

Habitat: ?

Vegetation: tropical deciduous forest.

Elevation: ?

Collection dates: 1929.IV.03; 1954.VII.21.

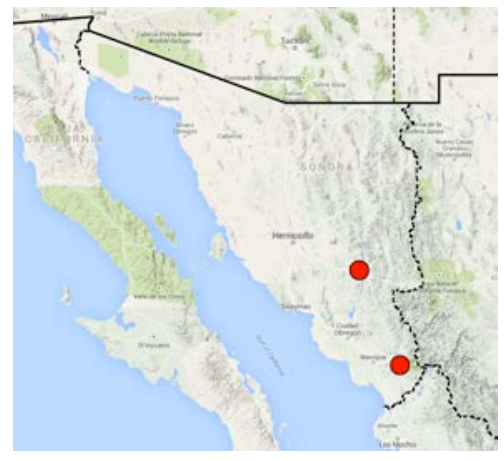

108. Paratachys spp. (may be more than one species)

Number examined: 51.

Municipalities: Agua Prieta, Álamos, Hermosillo, Ímuris, Moctezuma, Nacozari de García, Santa Ana, Yécora.

Habitat: marsh; rocky mountainside. [at light]

Vegetation: Chihuahuan and Sonoran desertscrub; foothills thornscrub; oak woodland; pine-oak forest; riparian forest; tropical deciduous forest.

Elevation range: $710-1830 \mathrm{~m}$.

Collection dates: 1953: VII.09-VII.16; 1960.VIII.12; 1967.

VII.27; 1982: VIII.19-VIII.20, VIII.23-VIII.24; 1983:

VII.11-VII.12; 1993: VII.06-VII.07; 2017.VIII.15.

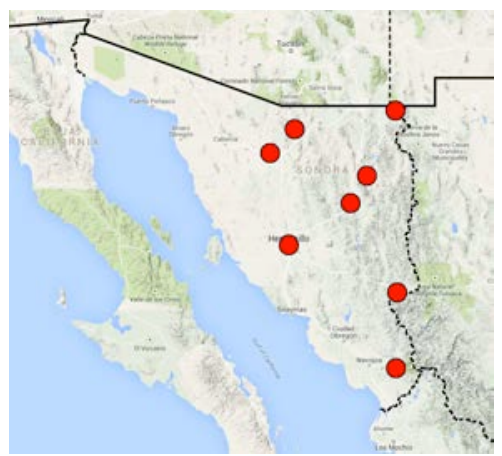

109. Pericompsus laetulus LeConte, 1852

Number examined: 2.

Municipality: Saric.

Habitat: ?

Vegetation: Sonoran desertscrub.

Elevation: ?

Collection date: 1929.VI.02. 


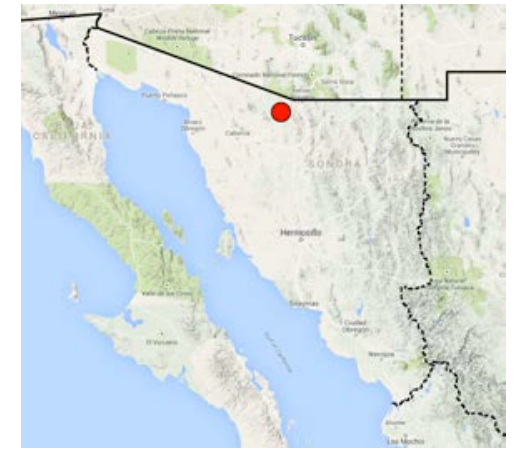

110. Pericompsus sellatus LeConte, 1852

Number examined: 1.

Municipality: Santa Ana.

Habitat: [at light]

Vegetation: Sonoran desertscrub.

Elevation: ?

Collection date: 1982.VIII.23.

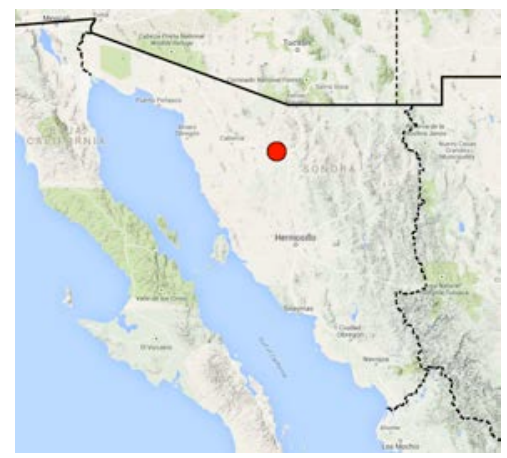

111. Pericompsus tlaloc Erwin, 1974

Number examined: 10.

Municipalities: Álamos, Guaymas, La Colorada.

Habitat: [at light]

Vegetation: Sonoran desertscrub; tropical deciduouis forest.

Elevation: ?

Collection dates: 1929.III.06; 1960: VIII.10, VIII.12; 1962.IV.16.

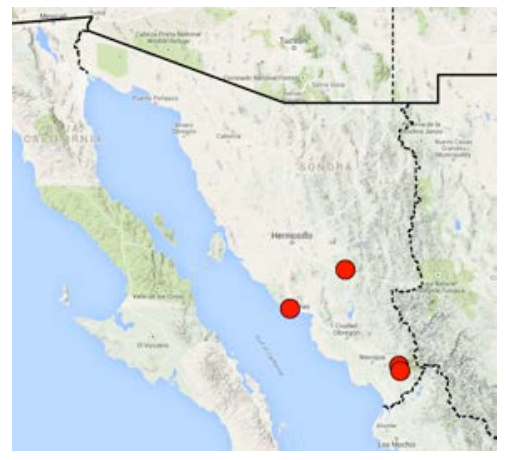

Number examined: 2

112. Pericompsus sp.

Municipalities: Ímuris, Santa Ana.

Habitat: [at light]

Vegetation: Sonoran desertscrub.

Elevation: ?

Collection dates: 1982.VIII.23; 1986.VIII.30.

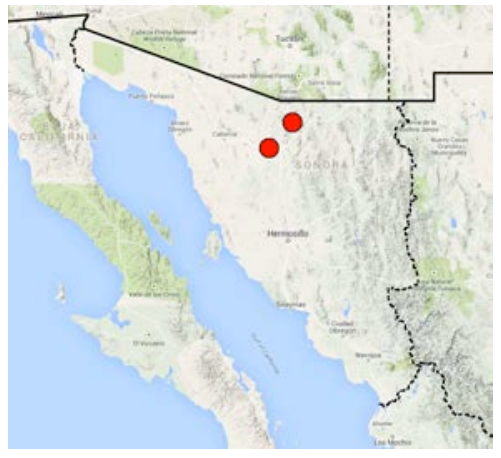

113. Polyderis sp.

Number examined: 7.

Municipalities: Álamos, Moctezuma, Santa Ana.

Habitat: [at light]

Vegetation: foothills thornscrub; Sonoran desertscrub; tropical deciduous forest.

Elevation range: $710-944 \mathrm{~m}$.

Collection dates: 1960.VIII.12; 1969.VIII.12; 1982. VIII.23; 1983: VII.11-VII.12.

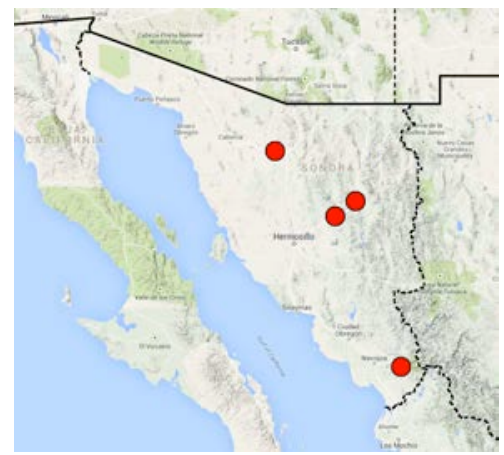

114. Tachys misellus (LaFerté-Sénectère, 1841) Number examined: 3 .

Municipality: Guaymas.

Habitat: ?

Vegetation: Sonoran desertscrub.

Elevation: ?

Collection date: 1973. . 


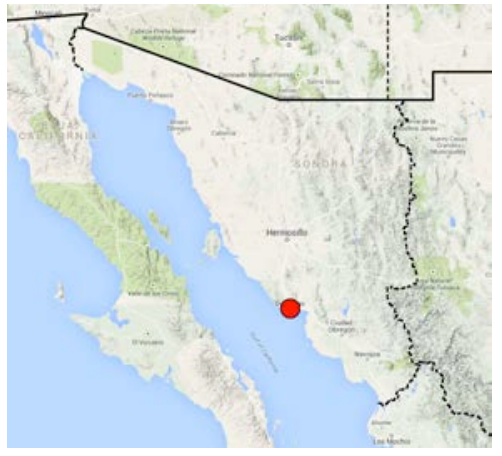

115. Tachys virgo LeConte, 1852

Number examined: 1.

Municipality: Guaymas.

Habitat: ?

Vegetation: Sonoran desertscrub.

Elevation: ?

Collection date: 1960.VIII.10.

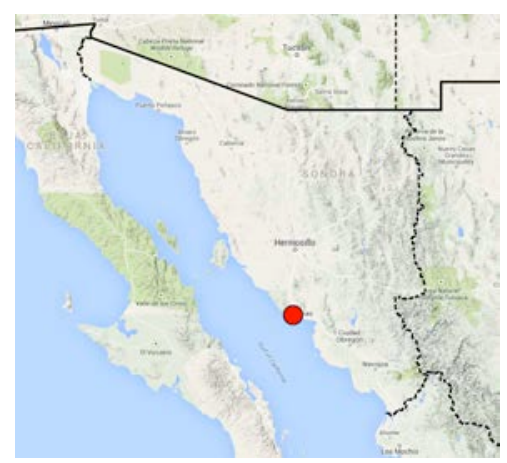

116. Tachys vittiger LeConte, 1852

Number examined: 5 .

Municipalities: Guaymas, Puerto Peñasco.

Habitat: ?

Vegetation: Sonoran desertscrub.

Elevation: ?

Collection dates: 1968.VI.14; 1973.V.

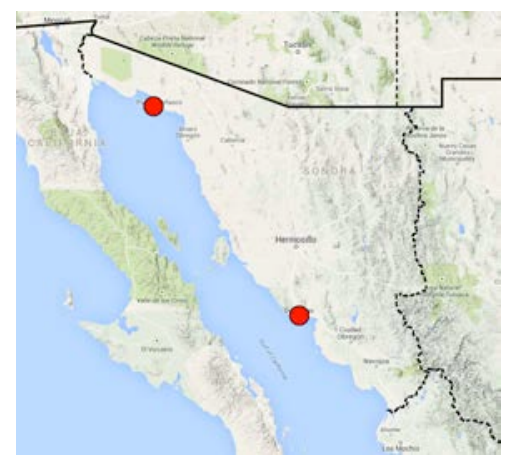

117. Tachys corax complex sp.

Number examined: 85 .

Municipalities: Guaymas, Huatabampito, Pitiquito.

Habitat: ?

Vegetation: coastal thornscrub; Sonoran desertscrub.
Elevation: ?

Collection dates: 1960.VIII.10; 1974: III.20, IV.22.

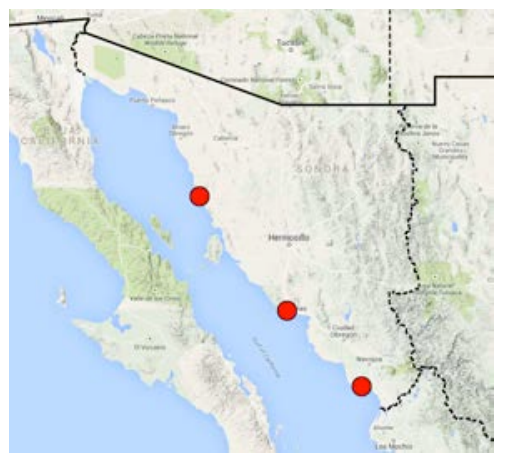

118. Tachys pallidus complex sp.

Number examined: 60 .

Municipalities: Guaymas, Huatabampito.

Habitat: ?

Vegetation: coastal thornscrub; Sonoran desertscrub. Elevation: ?

Collection dates: 1960.VIII.10; 1974.IV.22.

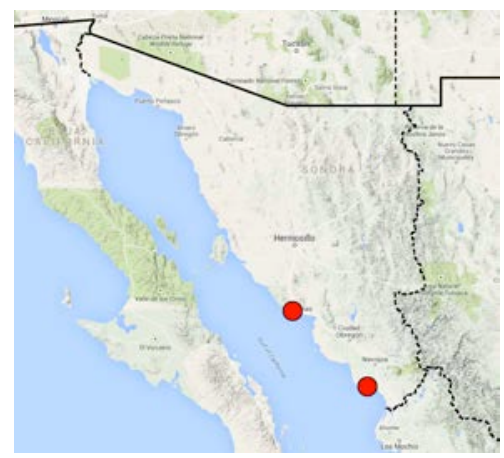

119. Tachys vittiger complex CASC $\mathrm{sp}$.

Number examined: 55.

Municipality: Guaymas.

Habitat: ?

Vegetation: Sonoran desertscrub.

Elevation: ?

Collection date: 1960.VIII.10,

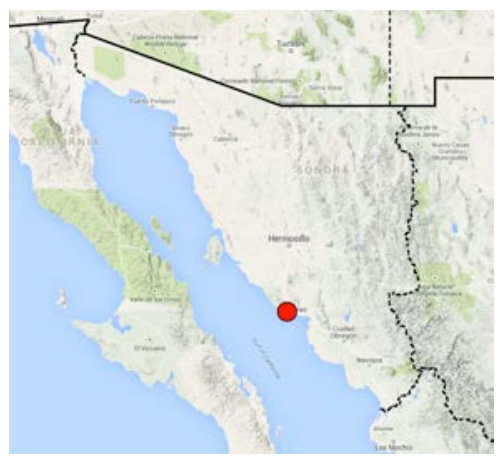


Number examined: 4 .

120. Tachys CASC n. sp. 1

Municipality: Guaymas.

Habitat: sand dunes.

Vegetation: coastal dunescrub; Sonoran desertscrub. Elevation:?

Collection date: 1974.IV.19.

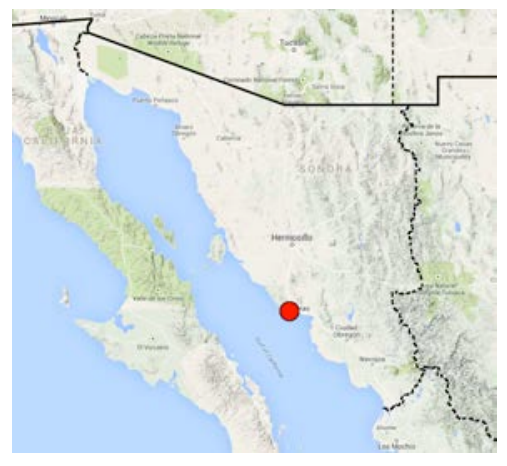

\section{Tachys CASC sp. 6}

Number examined: 74 .

Municipalities: Guaymas, Pitiquito.

Habitat: ?

Vegetation: Sonoran desertscrub.

Elevation: ?

Collection dates: 1953: VII.17-VII.31; 1960.08.10.

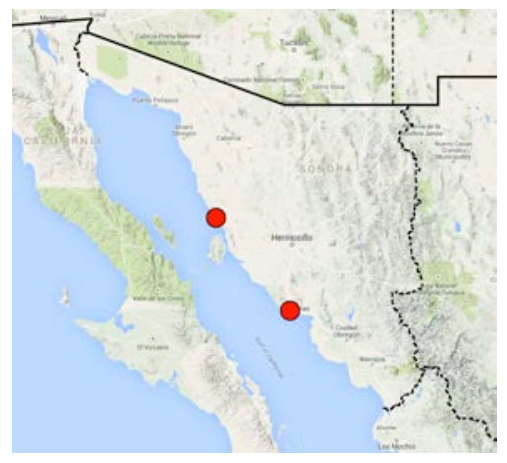

122. Tachys USNM sp. \#11

Number examined: 5 .

Municipality: Guaymas.

Habitat:?

Vegetation: Sonoran desertscrub.

Elevation: ?

Collection date: 1960.VIII.10.

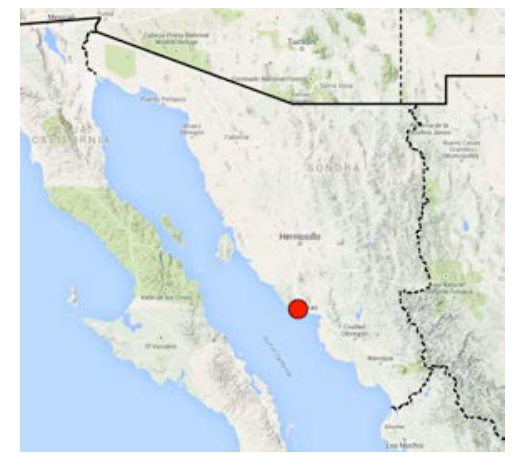

123. Tachys USNM sp. \#12

Number examined: 6 .

Municipalities: Guaymas, Pitiquito.

Habitat: ?

Vegetation: Sonoran desertscrub.

Elevation:?

Collection dates: 1953: VIII.20-VIII.31, IX.01-IX.10; 1960.08.10.

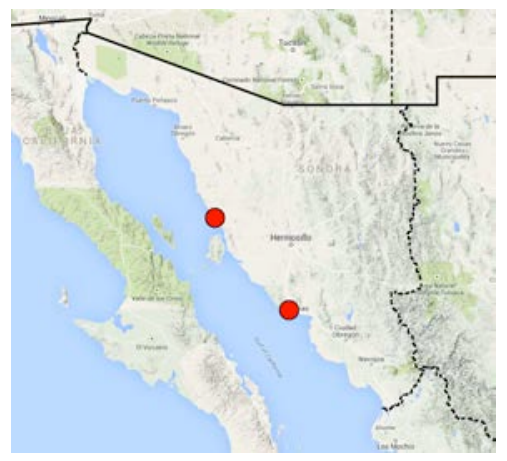

124. Tachys USNM sp. \#13

Number examined: 2.

Municipality: Guaymas.

Habitat: ?

Vegetation: Sonoran desertscrub.

Elevation: ?

Collection date: 1960.08.10.

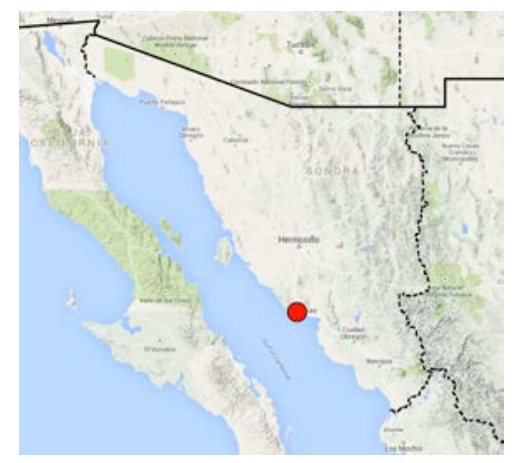


125. Tachys spp. (may be more than one species)

Number examined: 24 .

Municipalities: Cajeme, Empalme, Guaymas, Pitiquito.

Habitat: ?

Vegetation: coastal thornscrub; Sonoran desertscrub.

Elevation:?

Collection dates: 1952.VII.29; 1954.VIII.24; 1960.08.10; 1965.VIII.01; 1973.V; 1974.VIII.28.

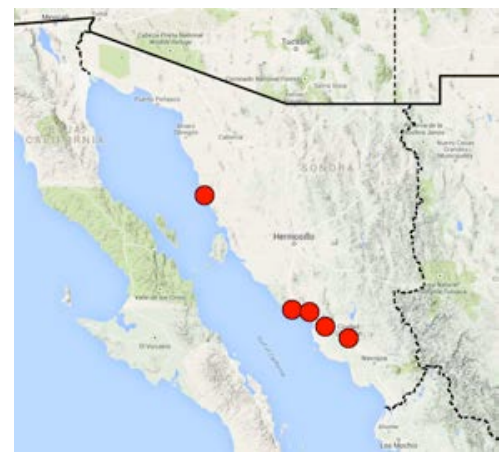

126. Tachyta nana inornata (Say, 1823)

Number examined: 3 .

Municipality: Nácori Chico.

Habitat: ?

Vegetation: pine-oak forest.

Elevation range: $1950-2000 \mathrm{~m}$.

Collection dates: 1983. VII.06; 2018.VIII.06.

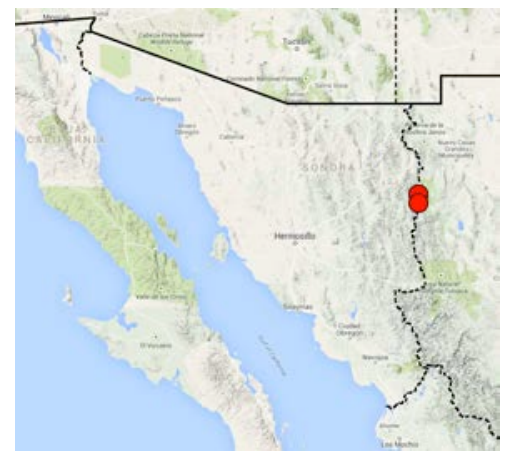

127. Subtribe Tachyina, New Genus, new species Number examined: 1 .

Municipality: Yécora.

Habitat: [at light]

Vegetation: pine-oak forest.

Elevation: $1547 \mathrm{~m}$.

Collection date: 1990: VI.30-VII.01.

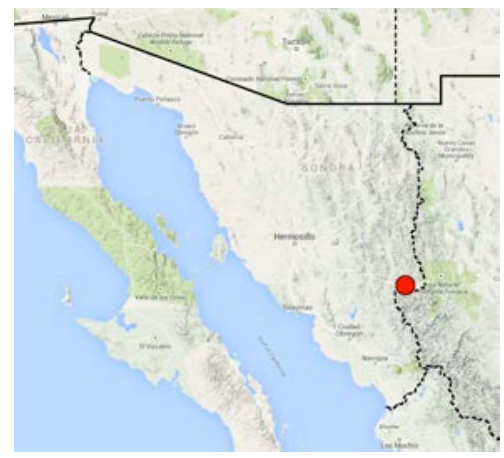

\section{Paussini}

128. Pachyteles gyllenhali (Dejean, 1825)

Number examined: 12 .

Municipalities: Agua Prieta, Hermosillo.

Habitat: [at light]

Vegetation: Sonoran desertscrub.

Elevation: $1350 \mathrm{~m}$.

Collection dates: 1980.X.25; 1982: VIII.19-VIII.21.

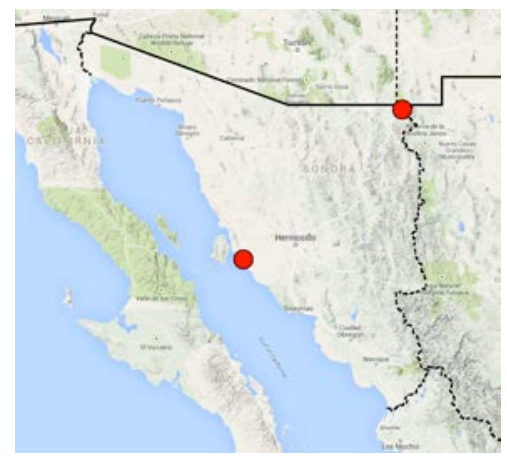

129. Pachyteles kuntzeni (Bänninger, 1927)

Number examined: 2 .

Municipality: Ures.

Habitat: urban [at light]

Vegetation: foothills thornscrub.

Elevation: $1377 \mathrm{~m}$.

Collection dates: 2014: VII.27-VII.29.

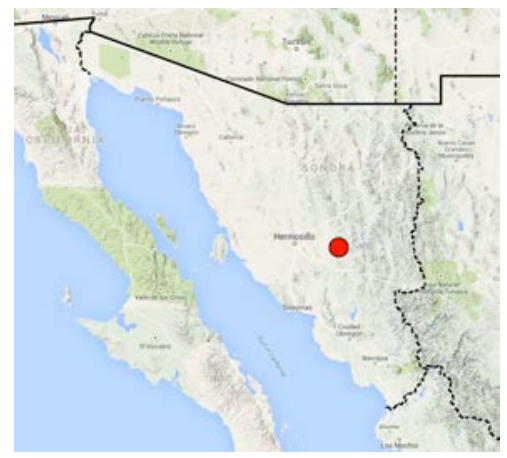


130. Pachyteles parca LeConte, 1884

Number examined: 7.

Municipalities: Álamos, Moctezuma, Ónavas, San Felipe de Jesús.

Habitat: ?

Vegetation: foothills thornscrub; tropical deciduous forest. Elevation range: $435-1300 \mathrm{~m}$.

Collection dates: 1982.VI.10; 1987: VII.26-VII.27, VII.30VII.31; 2019: VII.25, VIII.04.

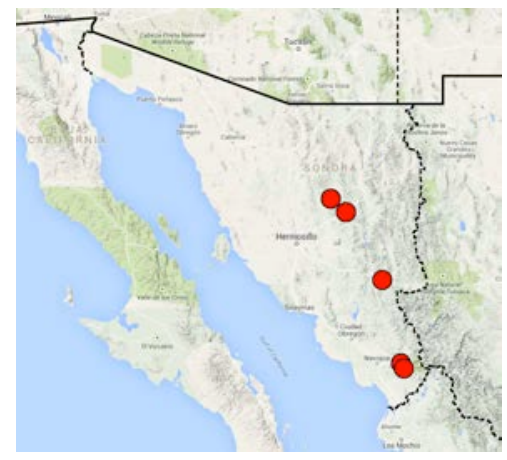

\section{Brachinini}

131. Brachinus adustipennis Erwin, 1969

Number examined: 1.

Municipality: Mazatán.

Habitat: ?

Vegetation: foothills thornscrub.

Elevation: $550 \mathrm{~m}$.

Collection date: 2012.VIII.13.

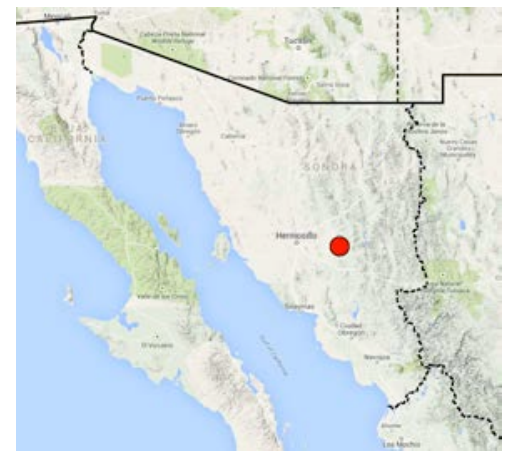

132. Brachinus azureipennis Chaudoir, 1876

Number examined: 1.

Municipality: Cananea.

Habitat: ?

Vegetation: desert grassland.

Elevation: $1531 \mathrm{~m}$.

Collection date: 2015.IX.06.

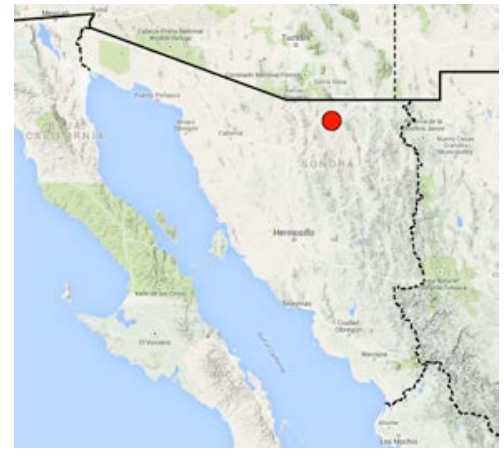

133. Brachinus cibolensis Erwin, 1970

Number examined: 2.

Municipality: Agua Prieta.

Habitat: [at light]

Vegetation: Chihuahuan desertscrub.

Elevation: $1350 \mathrm{~m}$

Collection dates: 1982: VIII.19-VIII.20.

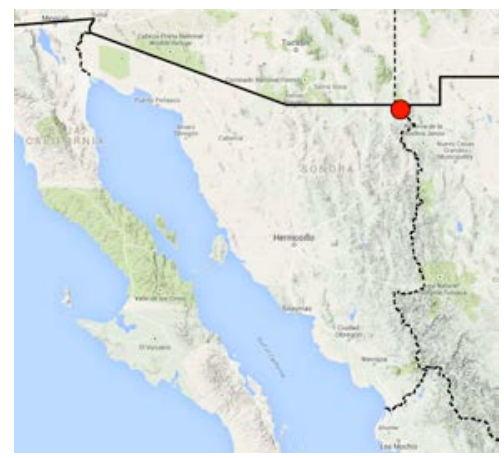

134. Brachinus consanguineus Chaudoir, 1876

Number examined: 3 .

Municipality: Álamos.

Habitat: [at light]

Vegetation: tropical deciduous forest.

Elevation: $1577 \mathrm{~m}$.

Collection dates: 1935.VII.1; 1989: VII.11-VII.13.

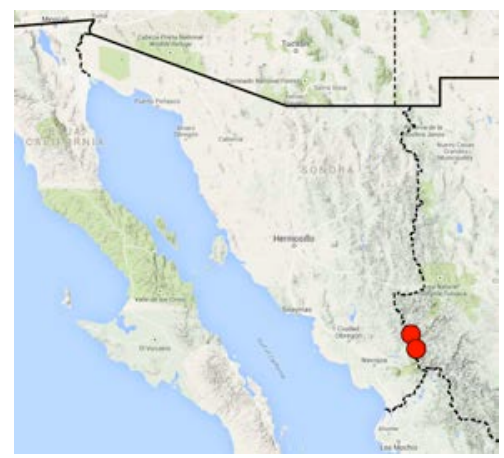

135. Brachinus costipennis Motchulsky, 1859

Number examined: 63.

Municipalities: Agua Prieta, Hermosillo, Nácori Chico, Santa Ana.

Habitat: river margin. [at light] 
Vegetation: Chihuahuan and Sonora desertscrub; oak woodland.

Elevation range: $1350-1450 \mathrm{~m}$.

Collection dates: 1897.IV.19; 1982: VIII.19-VIII.20; 1983. VII.04; 1985: VIII.01-VIII.02.

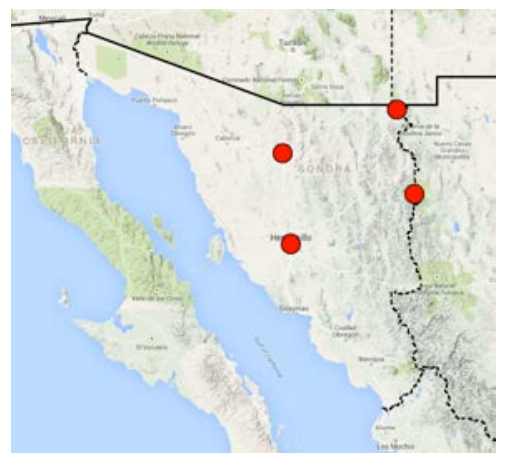

136. Brachinus elongatulus Chaudoir, 1876

Number examined: 949.

Municipalities: Aconchi, Agua Prieta, Álamos, Bacadéhuachi, Bacanora, Bacoachi, Cananea, Divisaderos, Fronteras, Granados, Hermosillo, Huachinera, Huásabas, Ímuris, Magdalena de Kino, Moctezuma, Naco, Nácori Chico, Nacozari de García, Nogales, Ónavas, Santa Ana, Santa Cruz, Yécora.

Habitat: cow pasture; rocky arroyo, canyon, slope, and mountainside; ridge top; roadside; river bed; urban. [at light]

Vegetation: Chihuahuan and Sonoran desertscrub; cottonwood-willow riparian forest; cypress-maple-alder-sycamore riparian forest; desert grassland; foothills thornscrub; oak woodland; pine-oak forest; riparian tropical scrub; tropical deciduous forest.

Elevation range: $293-2441 \mathrm{~m}$.

Collection dates: 1935: I.07, I.27; 1960.VIII.12; 1966. VIII.29; 1980.IX.23; 1982: VIII.01, VIII.03-VIII.07, VIII.09, VIII.11, VIII.19-VIII.20; 1983: VII-03-VII.06, VII.11-VII.12; 1985.VIII.01; 1986: VIII.29-VIII.30; 1987: VII.26-VII.27, VII.30-VII.31; 1989: VII.11VII.13; 1990: VI.28-VII.03, VIII.07-VIII.11; 1993: VII.05-VII.09; 2012: III.30, VII.07, VII.13, VII.21, VIII.01, VIII.09, IX.02, IX.12; 2013: VII.16, VIII.04, VIII.09, IX.10, IX.28; 2014: VII.28, VIII.02, VIII.24, VIII.27, IX.08, IX.14, VI.16, IX.20; 2015: VII.12, VIII.10, VIII.12-VIII.13, IX.06, IX.13-IX.14; 2016: V.01, VII.02, VIII.13-VIII.16, VIII.24, VIII.26, X.17; XI.20-XI.21; 2017.IV.23, V.23, VII-15-VII-16, VII.23, VIII.12-VIII.16, IX.16-IX.17; 2018: III.18-III.20; VI.27, VI.29, VIII.05-VIII.09, VIII.11, VIII.13; 2019: IV.26, IX.25.

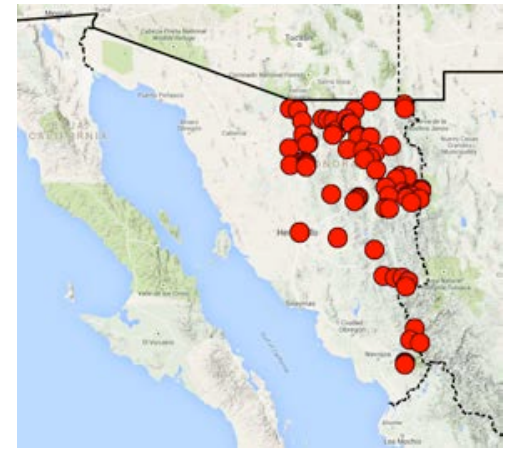

137. Brachinus explosus Erwin, 1970

Number examined: 1.

Municipality: Mazatán.

Habitat: gentle granitic slopes.

Vegetation: oak woodland.

Elevation: $1381 \mathrm{~m}$.

Collection date: 2014.VII.28.

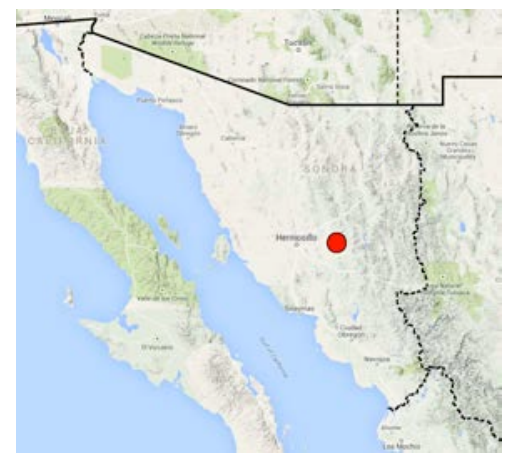

138. Brachinus favicollis Erwin, 1965

Number examined: 9 .

Municipality: Moctezuma.

Habitat: foothills thornscrub.

Vegetation:

Elevation: $944 \mathrm{~m}$.

Collection date: 1982.VIII.01.

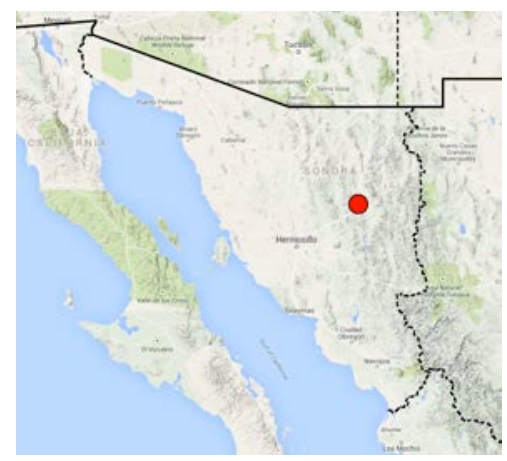


139. Brachinus fumans (Fabricius, 1781)

Number examined: 3 .

Municipalities: Álamos, Agua Prieta, Yécora.

Habitat: gentle rocky slope. [at light]

Vegetation: Chihuahuan desertscrub; pine-oak forest; tropical deciduous forest.

Elevation range: $1206-1582 \mathrm{~m}$.

Collection dates: 1935.X.15; 2014.IX.20; 2015.VIII.08.

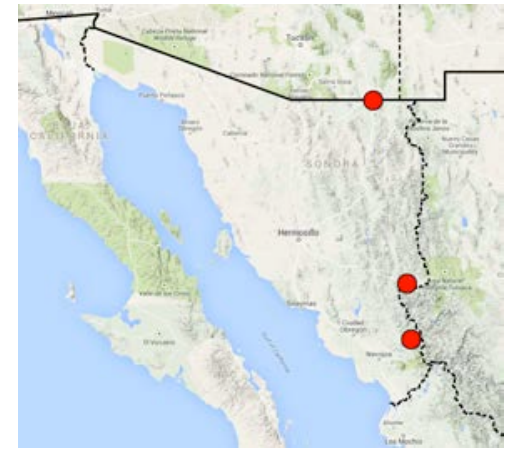

140. Brachinus galactoderus Erwin, 1970

Number examined: 7.

Municipality: Álamos.

Habitat: ?

Vegetation: tropical deciduous forest.

Elevation: $400 \mathrm{~m}$

Collection date: 1935.I.27.

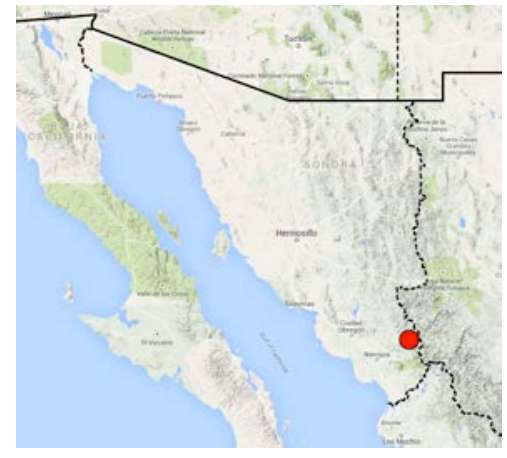

141. Brachinus gebhardis Erwin, 1965

Number examined: 1.

Municipality: Nacozari de García.

Habitat: [at light]

Vegetation: desert grassland.

Elevation: $1381 \mathrm{~m}$.

Collection date: 2015.VIII.08.

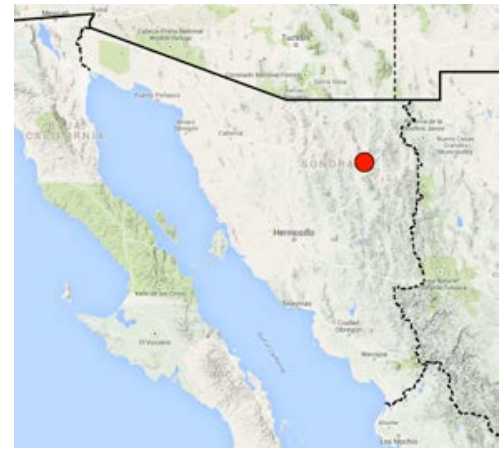

142. Brachinus hirsutus Bates, 1884

Number examined: 25 .

Municipalities: General Plutarco Elia Calles, Moctezuma, Nácori Chico, Santa Ana.

Habitat: stram margin.

Vegetation: foothills thornscrub; oak woodland; riparian tropical scrub; Sonoran desertscrub.

Elevation range: $457-1450 \mathrm{~m}$.

Collection dates: 1949.IV.04; 1982.VIII.01; 1983.VII.05; 1985: VIII.01-VIII.02.

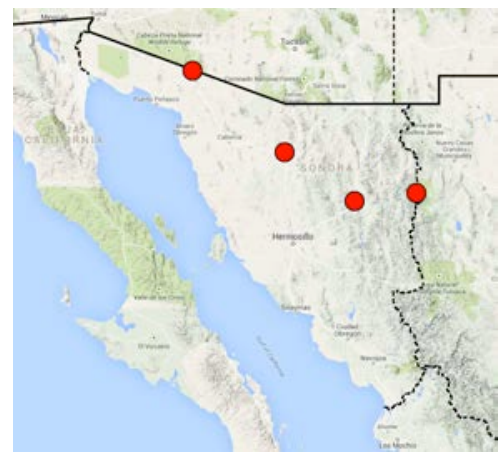

143. Brachinus imperialensis Erwin, 1965

Number examined: 1.

Municipality: Hermosillo.

Habitat: ?

Vegetation: Sonoran desertscrub.

Elevation: ?

Collection date: 1953: VIII.15-VIII.20.

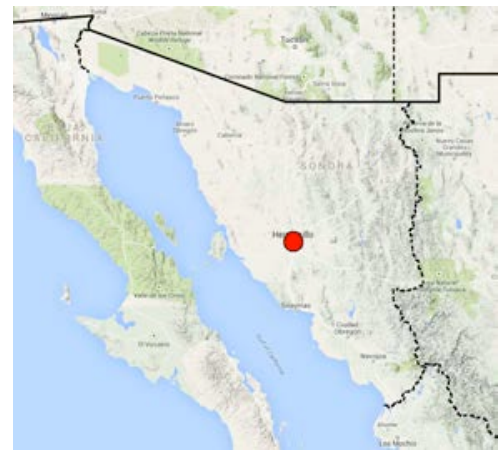


144. Brachinus lateralis Dejean, 1831

Number examined: 10.

Municipalities: Empalme, Ímuris, Moctezuma, Nacozari de García, Santa Ana, Ures, Villa Pesqueira.

Habitat: rocky granitic slope.

Vegetation: Sonoran desertscrub; desert grassland; oak woodland; riparian forest; coastal and foothills thornscrub.

Elevation range: $30-1422 \mathrm{~m}$.

Collection dates: 1929.II.24; 1957.IV.03; 1958.IV.03; 1962.V.01; 1965.VII.31; 1982: VIII.11, VIII.24; 1985. VIII.01; 1993.VII.04; 2014.IV.27.

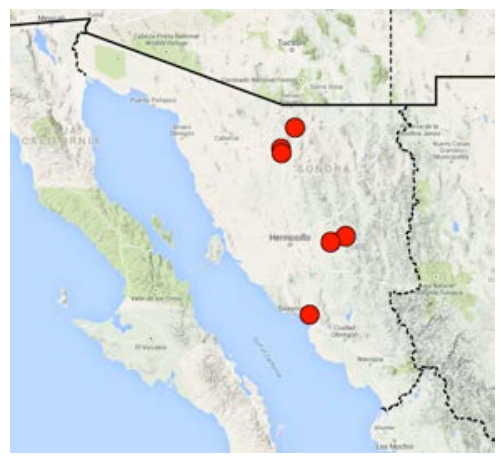

145. Brachinus mexicanus Dejean, 1831

Number examined: 50.

Municipalities: Agua Prieta, Álamos, Bacanora, Bacerac, Huásabas, Ímuris, Moctezuma, Nácori Chico, Santa Ana, Villa Pesqueira, Yécora.

Habitat: river margin; rocky slope; stream margin; urban. [at light]

Vegetation: Chihuahuan and Sonora desertscrub; foothills thornscrub; oak woodland; pine-oak forest; riparian forest; riparian tropical scrub; tropical deciduous forest.

Elevation range: $561-1830 \mathrm{~m}$.

Collection dates: 1929.II.24; 1935.I.27; 1982: VIII.01, VIII.07-VIII.08, VIII.20; 1983.VII.05; 1985: VIII.01VIII.02; 1993: VII.06-VII.07, VIII.07-VIII.11; 2012:VIII.02; 2014.VIII.02; 2015.VII.12.

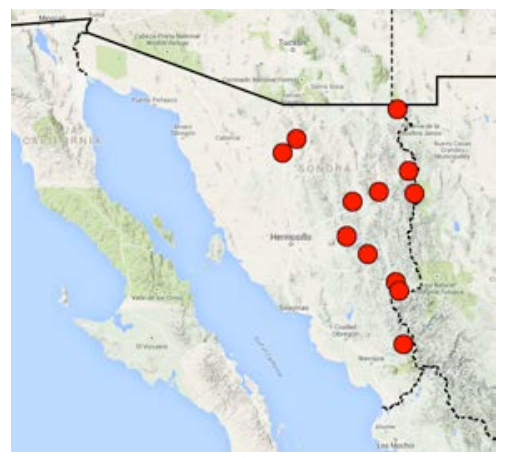

146. Brachinus sonorous Erwin, 1970

Number examined: 2.

Municipality: Empalme.

Habitat: ?

Vegetation: coastal thornscrub.

Elevation: $30 \mathrm{~m}$.

Collection date: 1962.V.01.

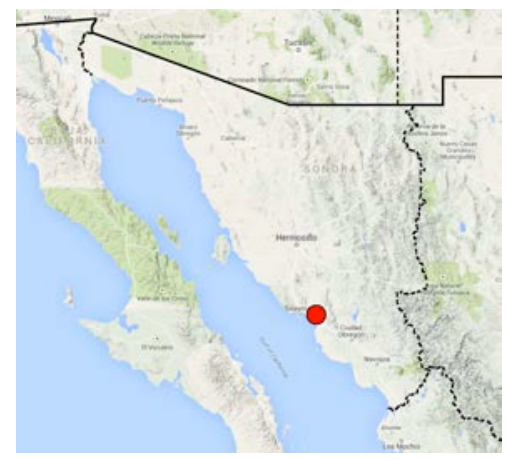

16. Morionini

147. Morion aridus Allen, 1968

Number examined: 5 .

Municipalities: Álamos, Bacanora, Guaymas, Pitiquito.

Habitat: rocky slope. [at light]

Vegetation: foothills thornscrub; Sonoran desertscrub; tropical deciduous forest.

Elevation range: $518-1387 \mathrm{~m}$.

Collection dates: 1953: IX.01-IX.10; 1960.VIII.10; 1987: VII.30, VII.31; 2014.VIII.02.

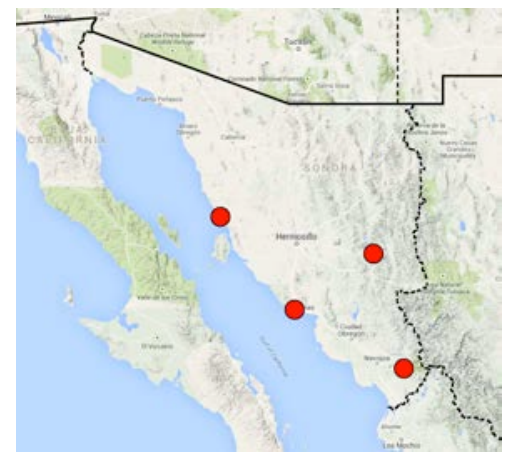

148. Morion cordatus (Chaudoir, 1837)

Number examined: 6 .

Municipalities: Álamos, Moctezuma, Ónavas.

Habitat: Atta nest.

Vegetation: foothills thornscrub; oak woodland; tropical deciduous forest.

Elevation range: $518-944 \mathrm{~m}$.

Collection dates: 1983: VII.11, VII.12; 1987: VII.26VII.27, VII.30-VII.31. 


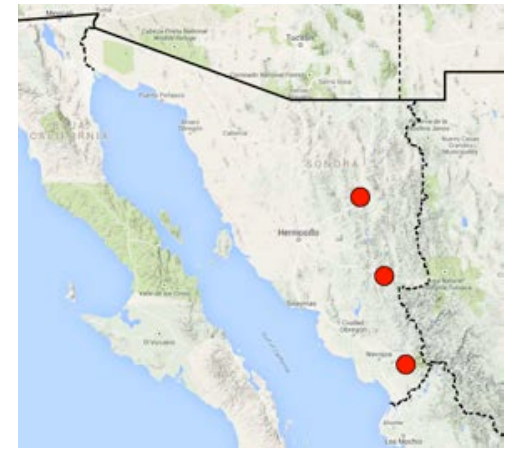

149. Morion lafertei Guérin-Meneville, 1844

Number examined: 1.

Municipality: Altar.

Habitat: in Atta colony.

Vegetation: Sonoran desertscrub.

Elevation: ?

Collection date: 1964.XI.29.

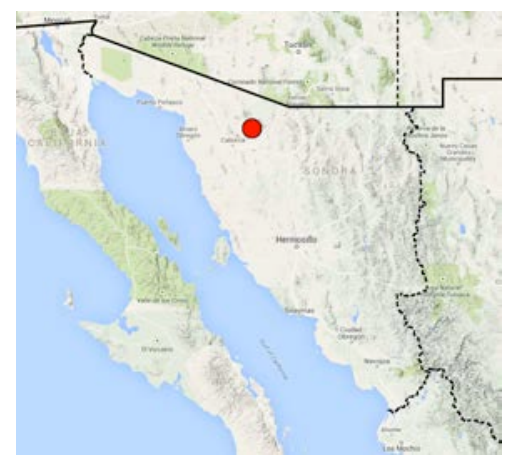

17. Abacetini

150. Oxycrepis infimus (Bates, 1882)

Number examined: 2 .

Municipalities: Carbó, Santa Ana.

Habitat: ?

Vegetation: Sonoran desertscrub.

Elevation: ?

Collection dates: 1958.IV.03; 1965.VII.31.

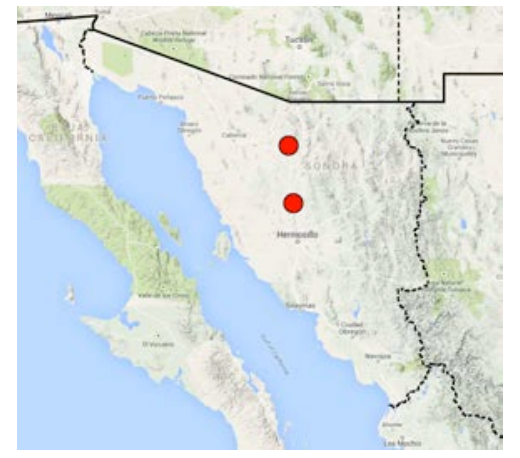

151. Oxycrepis pactinullus (Allen, 1972)

Number examined: 2.

Municipalities: Álamos, Carbó.

Habitat: ?

Vegetation: Sonoran desertscrub; tropical deciduous forest. Elevation: ?

Collection dates: 1964.VIII.24; 1965.VII.31.

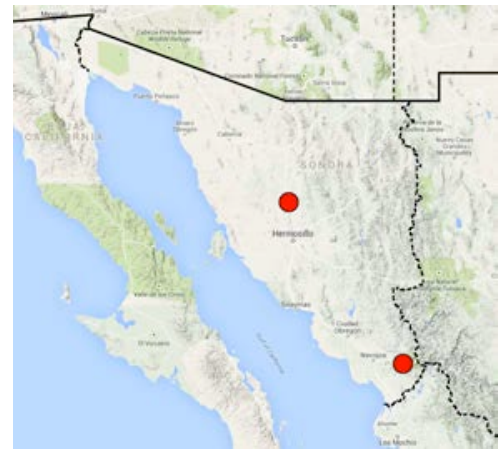

152. Oxycrepis sculptilis (Bates, 1884)

Number examined: 3 .

Municipalities: Álamos, Navojoa.

Habitat: [at light]

Vegetation: coastal thornscrub; tropical deciduous forest.

Elevation: ?

Collection dates: 1964: VIII.24-VIII.25.

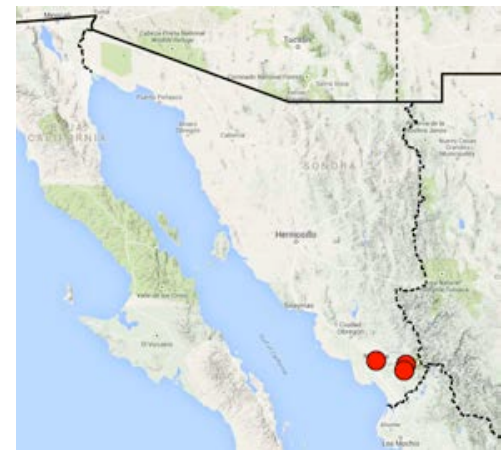

18. Pterostichini

153. Abaris splendidula (LeConte, 1863)

Number examined: 60 .

Municipalities: Agua Prieta, Ímuris, Moctezuma, Nacozari de García.

Habitat: rocky arroyo.

Vegetation: Chihuahuan desertscrub; foothills thornscrub; oak woodland; riparian tropical scrub.

Elevation range: $500-1460 \mathrm{~m}$.

Collection dates: 1982: VIII.01, VIII.02, VIII.24; 2013. VIII.23; 2017.VIII.14. 


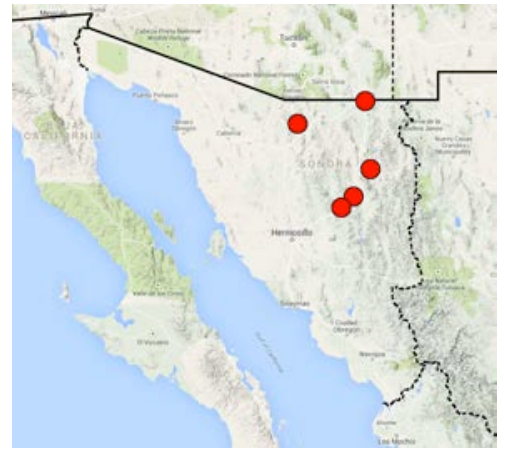

154. Cyclotrachelus substriatus (LeConte, 1848)

Number examined: 13.

Municipality: Nácori Chico

Habitat: [at light]

Vegetation: cypress-maple-alder-sycamore riparian forest; pine-oak forest.

Elevation range: $1648-2330 \mathrm{~m}$.

Collection dates: 1982: VIII.06-VII.08; 1983.VII.06; 2018.VIII.09.

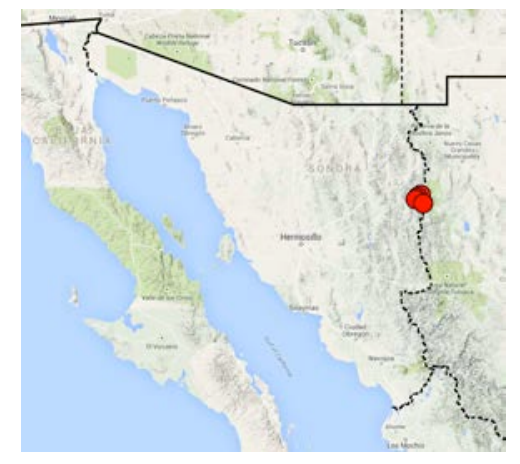

155. Hybothecus flohri (Bates, 1882)

Number examined: 15.

Municipalities: Álamos, Fronteras, Ímuris, Moctezuma.

Habitat: [at light]

Vegetation: Chihuahuan desertscrub; foothills thornscrub; riparian tropical scrub; tropical deciduous forest.

Elevation range: $400-1202 \mathrm{~m}$.

Collection dates: 1982: VIII.01, VIII.02, VIII.24; 1987: VII.30, VII.31; 1993.VII.04; 2012: VIII.01, IX.12.

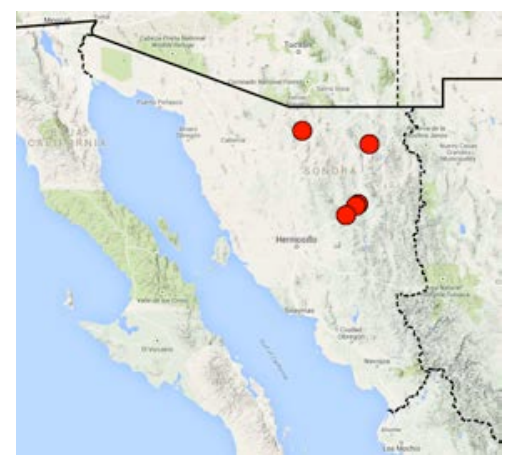

156. Poecilus diplophryus Chaudoir, 1876

Number examined: 3

Municipality: Guaymas.

Habitat: [at light]

Vegetation: Sonoran desertscrub.

Elevation: $46 \mathrm{~m}$.

Collection date: 1984.VII.02.

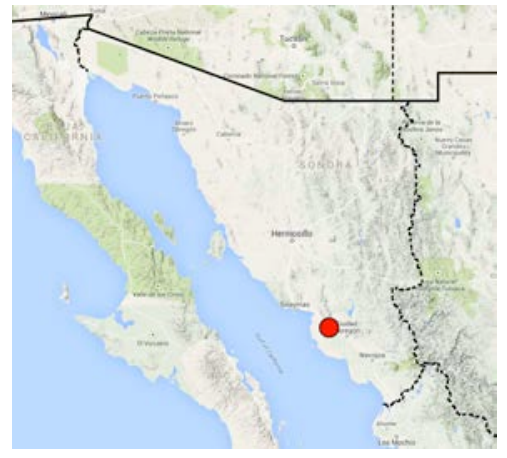

157. Poecilus scitulus LeConte, 1848

Number examined: 10.

Municipalities: Benjamín Hill; Carbó, Fronteras, Hermosillo, Nácori Chico, Santa Ana.

Habitat: [at light]

Vegetation: Chihuahuan and Sonoran desertscrub; foothills thornscrub; stock tank.

Elevation range: $175-1450 \mathrm{~m}$.

Collection dates: 1965.VII.31; 1983.VII.04; 2012.VIII.01; 2017.VII.23; 2020.IX.10.

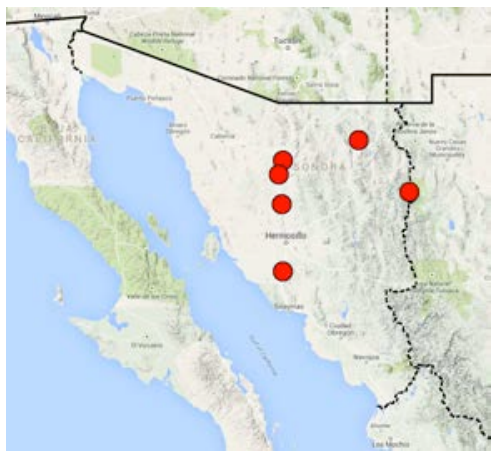

\section{Zabrini}

158. Amara californica Dejean, 1828

Number examined: 69 .

Municipalities: Agua Prieta, Bacadéhuachi, Divisaderos, Fronteras, Granados, Huachinera, Ímuris, Nácori Chico, Nacozari de García, Nogales, Santa Cruz, Saric, Yécora.

Habitat: large flat area near ridge top; rocky arroyo; rocky mountainside; shallow rocky arroyo.

Vegetation: Chihuahuan and Sonoran desertscrub; desert grassland; foothills thornscrub; oak woodland; pine-oak forest; sycamore riparian forest.

Elevation range: $502-2441$.

Collection dates: 1929: VI.02, VII.03; 1965.VII.31; 1982:

VIII.03, VIII.04, VIII.06, VIII.07, VIII.09, VIII.24; 
1983: VII.04, VII.07; 1986.VIII.30; 1990.VIII.11. 1993: VII.06-VII.07; 2015: VIII.11-VIII.12; 2016: IV.16, VIII.15, XI.21; 2017: IV.23, VIII, VIII.13, VIII.15; 2019: IV.24-IV.26.

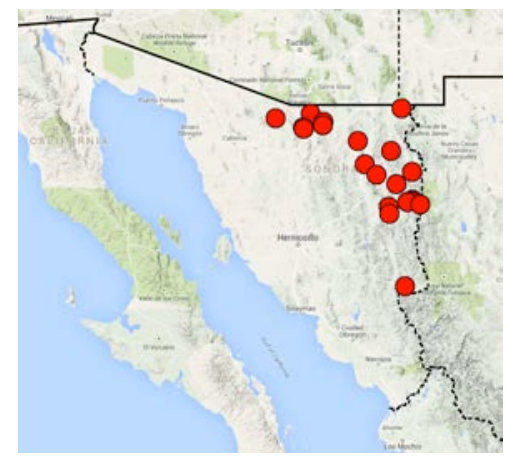

159. Amara deparca (Say, 1830)

Number examined: 3 .

Municipalities: Agua Prieta, Nogales.

Habitat: ?

Vegetation: Chihuahuan desertscrub; desert grassland. Elevation: $1350 \mathrm{~m}$.

Collection dates: 1965.VII.30; 1982.VIII.20.

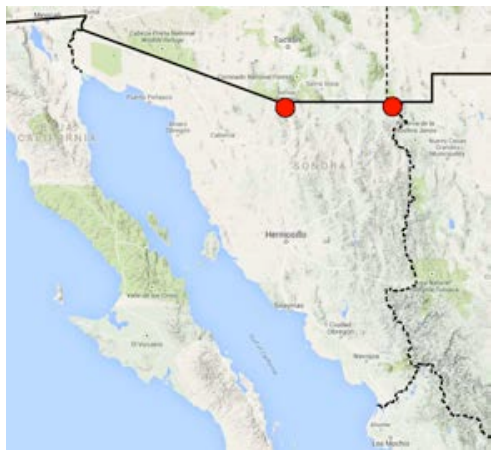

160. Amara nupera G. Horn, 1892

Number examined: 3 .

Municipalities: Agua Prieta, Cananea, Nácori Chico.

Habitat: rocky slope.

Vegetation: Chihuahuan desertscrub; desert grassland; riparian deciduous forest; pine-oak forest.

Elevation range: $1291-2422 \mathrm{~m}$.

Collection dates: 1983.VII.06; 2013.VIII.02; 2017.III.29.

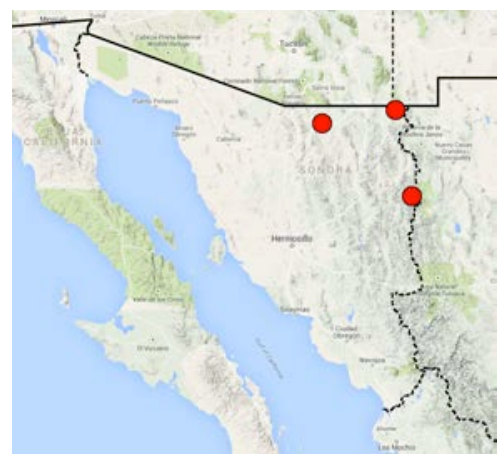

161. Amara sera Say, 1830

Number examined: 1.

Municipality: Nácori Chico.

Habitat: camp clearing.

Vegetation: cypress-maple-alder-sycamore riparian forest. Elevation: $1648 \mathrm{~m}$.

Collection date: 2018.VIII.09.

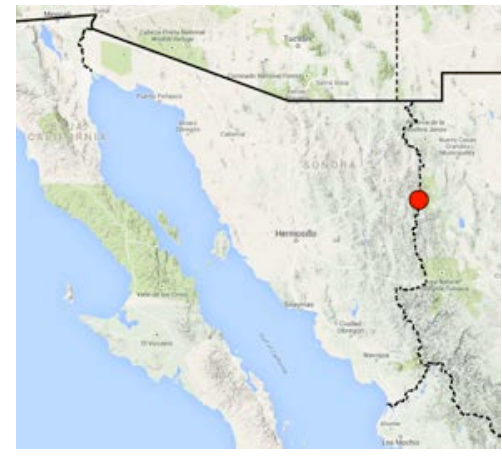

162. Amara sp.

Number examined: 1.

Municipality: Yécora.

Habitat: litter

Vegetation: oak woodland.

Elevation: $1680 \mathrm{~m}$.

Collection date: 1993: VII.09.

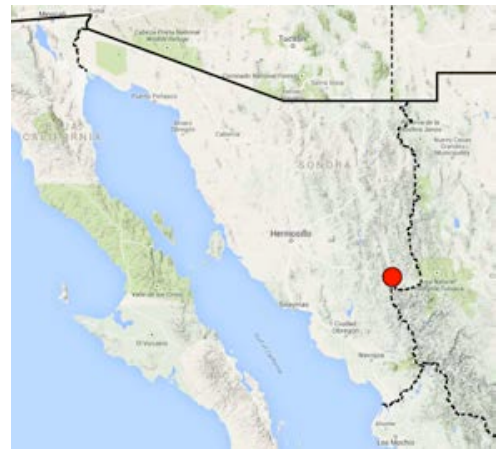

20. Oodini

163. Oodinus alutaceus (Bates, 1882)

Number examined: 2 .

Municipality: Guaymas.

Habitat: ?

Vegetation: Sonoran desertscrub.

Elevation: $10 \mathrm{~m}$.

Collection dates: 1965.VIII.01; 1972.IX.03. 


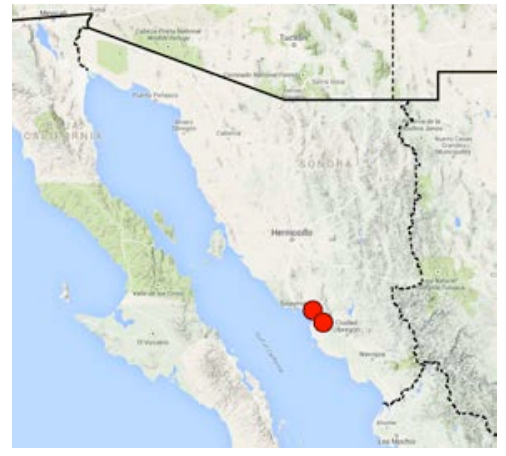

164. Stenocrepis elegans (LeConte, 1851)

Number examined: 16.

Municipalities: Álamos, Hermosillo.

Habitat: ?

Vegetation: Sonoran desertscrub; tropical deciduous forest.

Elevation: ?

Collection dates: 1953: VII.09-VII.16; 1960.VIII.12.

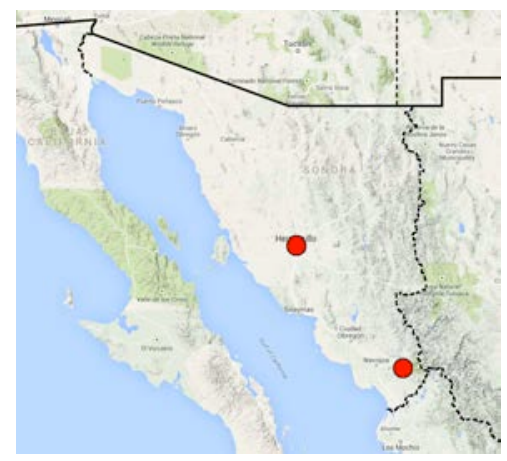

\section{Panagaeini}

165. Panagaeus sallei Chaudoir, 1862

Number examined: 9 .

Municipalities: Cananea, Carbó, Cucurpe, Fronteras, Granados, Mazatán, Yécora.

Habitat: rocky mountainside; urban. [at light]

Vegetation: Chihuahuan and Sonoran desertscrub; desert grassland; foothills thornscrub; oak woodland; pine-oak forest; riparian deciduous forest.

Elevation range: $540-1521 \mathrm{~m}$.

Collection dates: 1959.IX.08; 1987.VII.28-VII.29; 1998. VIII.04; 2012.VII.12; 2014.VIII.29; 2015.IX.06; 2016. VIII.16; 2017.VII.16; 2018.VIII.11.

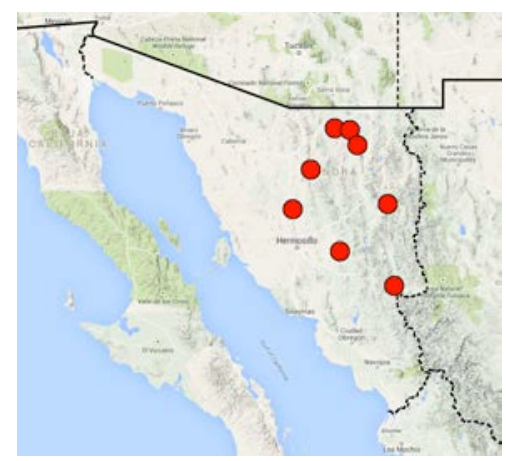

\section{Chlaeniini}

166. Chlaenius caeruleicollis Chaudoir, 1876

Number examined: 1 .

Municipality: Yécora

Habitat: ?

Vegetation: pine-oak forest.

Elevation: $1646 \mathrm{~m}$.

Collection date: 1990.VIII.07.

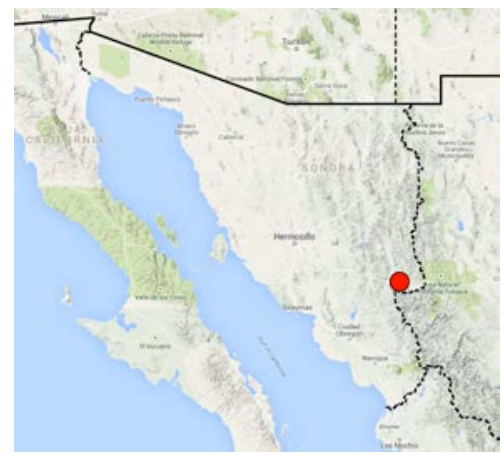

167. Chlaenius chaudoiri G. Horn, 1876

Number examined: 6 .

Municipalities: Álamos, Nacozari de García, Santa Ana.

Habitat: ?

Vegetation: Snoran desertscrub; oak woodland.

Elevation: ?

Collection dates: 1958.IV.03; 1978.VIII.10; 2019.VII.22.

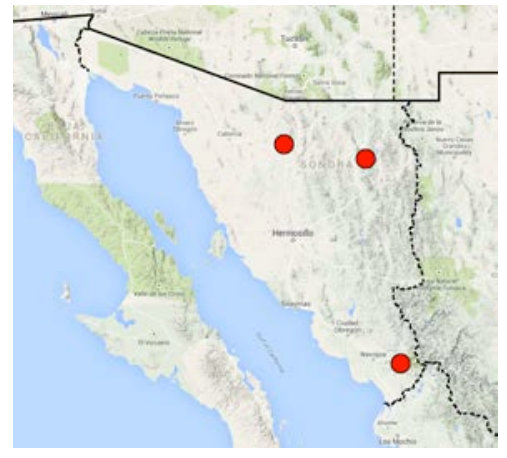

168. Chlaenius cumatilis LeConte, 1851

Number examined: 20.

Municipalities: Agua Prieta, Cananea, Divisaderos, Moctezuma, Nácori Chico, San Felipe de Jesús, Santa Ana.

Habitat: flood litter; rocky slope and stream margin. [at light]

Vegetation: Chihuahuan and Sonoran desertscrub; cottonwood-willow riparian forest; cypress-maple-alder-sycamore riparian forest; desert grassland; foothills thornscrub; oak woodland; pine-oak forest.

Elevation range: $930-1648 \mathrm{~m}$.

Collection dates: 1982: VIII.01, VIII.20; 1983.VII.05; 2014.VIII.27; 2016.XI.20; 2018: III.19-III.20, VIII.05, VIII.08-VIII.09; 2019.VII.04. 


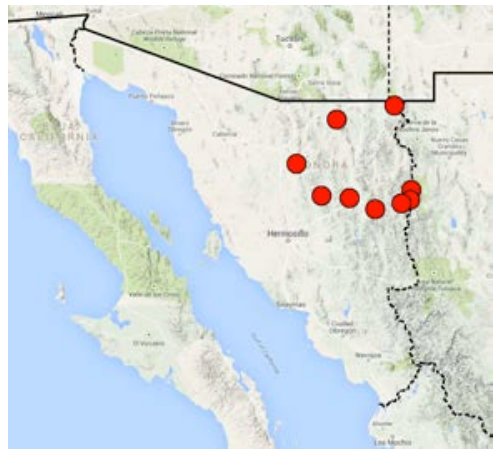

169. Chlaenius cursor Chevrolat, 1835

Number examined: 4.

Municipalities: Santa Ana, Yécora.

Habitat: ?

Vegetation: pine-oak forest; Sonoran deserscub.

Elevation: ?

Collection dates: 1985.VIII.01; 1993.VII.06.

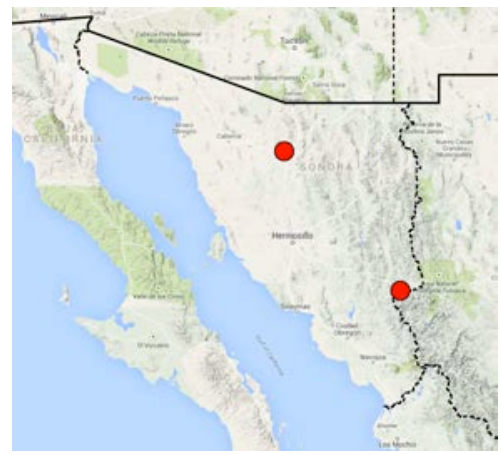

170. Chlaenius forreri Bates, 1884

Number examined: 64 .

Municipalities: Aconchi, Álamos, Arizpe, Bacadéhuachi, Cananea, Carbó, Cucurpe, Fronteras, Hermosillo, Huachinera, Ímuris, La Colorada, Magdalena de Kino, Mazatán, Moctezuma, Nácori Chico, Nacozari de García, Nogales, Navojoa, Ónavas, Yécora.

Habitat: cow pasture; disturbed area; rocky canyon, hillside, and mountainside; urban. [at light]

Vegetation: desert grassland; foothills thornscrub; oak woodland; pine-oak forest; Sonoran desertscrub; sycamore riparian deciduous forest; tropical deciduous forest.

Elevation range: $50-1969 \mathrm{~m}$.

Collection dates: 1982: VIII.01-VIII.03, VIII.05, VIII.09, VIII.11; 1983: VII.02, VII.04; 1987.IX.02; 1989.VII.08; 1990: VI.28-VI.29, VIII.11; 1993.VII.05; 2012: VI.26, VII.12, VIII.06, VIII.13; 2013: VII.02, IX.09; 2014: VIII.27, VIII.29, IX.14; 2015.VII.12; 2016.VII.02; 2017: VII.15, VII.21-VII.22; 2018.VI.27; 2019: VII.06, VII.25.

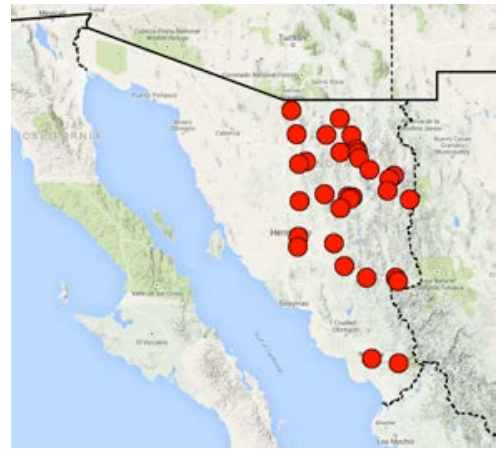

171. Chlaenius herbaceus Chevrolat, 1835

Number examined: 2.

Municipality: Navojoa.

Habitat: ?

Vegetation: coastal thornscrub.

Elevation: $50 \mathrm{~m}$.

Collection date: 1987.XI.02.

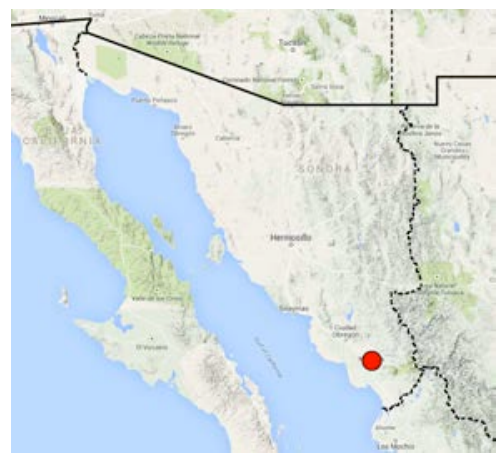

172. Chlaenius leucoscelis Chevrolat, 1834

Number examined: 18.

Municipalities: Agua Prieta, Baviácora, Cananea, Fronteras, Ímuris, Nácori Chico, Nacozari de García, Yécora.

Habitat: camp clearing; flood litter; rocky stream canyon, hillside, and mountainside. [at light]

Vegetation: Chihuahuan desertscrub; cottonwood-willow riparian deciduous forest; cypress-maple-alder-sycamore riparian forest; desert grassland; oak woodland; pine-oak forest.

Elevation Range: 945 - $1707 \mathrm{~m}$.

Collection dates: 1980.IX.21; 1982:VIII.20-VIII.21; 1983. VII.05; 1986.VIII.30; 1990.VIII.11; 2014.IX.14; 2016. VIII.15; 2017.VIII.15; 2018: VIII.05-VIII.06, VIII.08. 


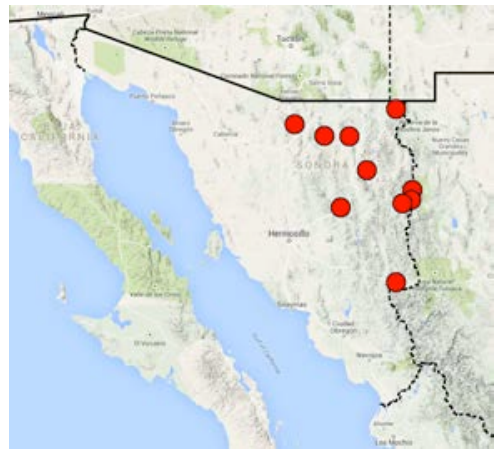

173. Chlaenius nebraskensis LeConte, 1856

Number examined: 1.

Municipality: Ímuris.

Habitat: ?

Vegetation: cottonwood-willow riparian deciduous forest.

Elevation: ?

Collection date: 1956.XII.26.

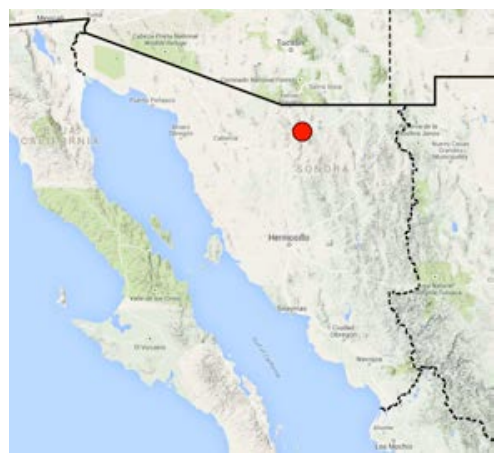

174. Chlaenius obsoletus LeConte, 1851

Number examined: 3 .

Municpalities: Agua Prieta, Hermosillo, Moctezuma.

Habitat: [at light]

Vegetation: foothills thornscrub; Chihuahuan and Sonoran desertscrub.

Elevation Range: 1000 - $1350 \mathrm{~m}$.

Collection dates: 1953: VII-09-VII.16; 1982: VIII.19VIII.20; 1993.VII.04.

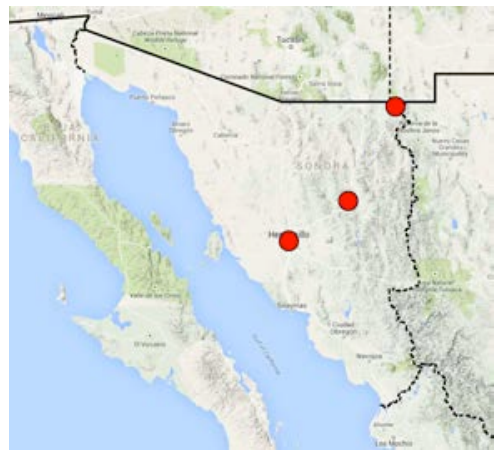

175. Chlaenius orbus G. Horn, 1871

Number examined: 68 .

Municipalities: Álamos, Bacadéhuachi, Cananea, Carbó,
Guaymas, Hermosillo, Ímuris, La Colorada, Magdalena de Kino, Mazatán, Rayón, Santa Ana.

Habitat: rocky canyon, slope, and mountainside; urban.

Vegetation: desert grassland; foothills thornscrub; pineoak forest; Sonoran desertscrub; sycamore-cypress riparian forest.

Elevation Range: 235 - $1680 \mathrm{~m}$.

Collection dates: 1953: VII.09-VII.16, VIII.15-VIII.20, IX.28; 1957: VII.28-VII.28; 1958.IV.03; 1984.VII.02; 1989.VII.08; 2012: VII.12, VIII.06, IX.05; 2014. VIII.29; 2015: VII.12, IX.06; 2017: VII.21-VII.23.

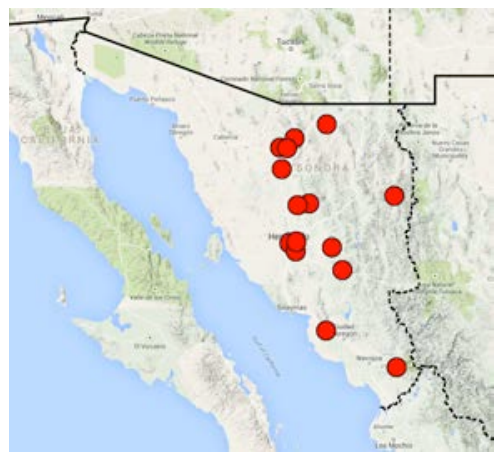

176. Chlaenius purpureus Chaudoir, 1876

Number examined: 12.

Municipalities: Aconchi, Álamos, Mazatán, Nacozari de García.

Habitat: rocky canyon, slopes, and mountainside. [at light] Vegetation: foothills hornscrub; oak forest; oak woodland; pine- sycamore riparian deciduous forest; tropical deciduous forest.

Elevation Range: $1301-1830 \mathrm{~m}$.

Collection dates: 1989: VII.11-VII.13; 1993: VII.06VII.07; 2013.VII.02; 2014.VII.28; 2015.VIII.08; 2017. VIII.15.

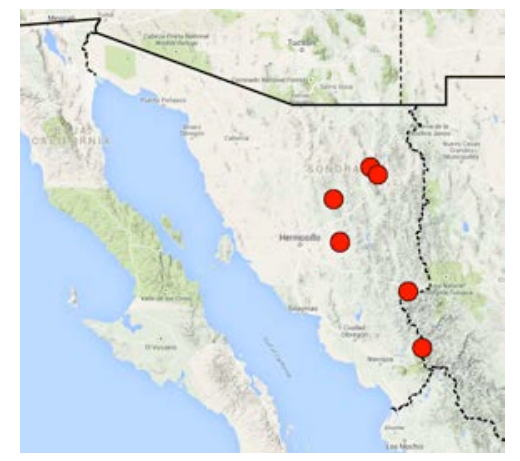

177. Chlaenius ruficauda Chaudoir, 1856

Number examined: 24.

Municipalities: Agua Prieta, Ímuris, Nácori Chico, San Felipe de Jesús, Santa Ana.

Habitat: stream margin.

Vegetation: Chihuahuan desertscrub; cottonwood-willow riparian forest; oak woodland.

Elevation Range: 963 - 1450 m. 
Collection dates: 1982.VIII.21; 1983.VII.05; 1985. VIII.01; 1986.VIII.30; 2019.VIII.05.

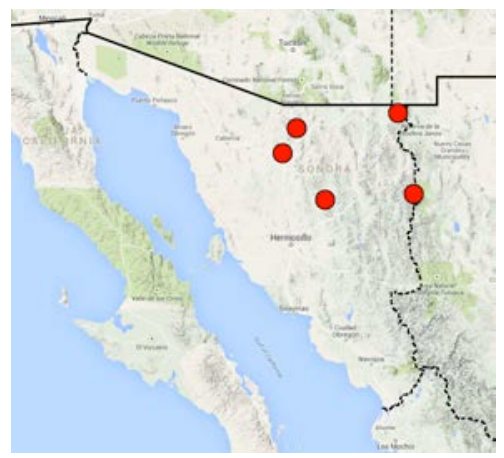

178. Chlaenius sparsus LeConte, 1863

Number examined: 18.

Municipalities: Álamos, Ímuris, Moctezuma, Nácori Chico, Navojoa, Yécora.

Habitat: camp clearing; roadside; rocky stream canyon.

Vegetation: cypress-maple-alder-sycamore riparian forest; foothills thornscrub; oak woodland; pine-oak forest; tropical deciduous forest.

Elevation Range: $50-1900 \mathrm{~m}$.

Collection dates: 1956.XII.26; 1987.XI.02; 1989.VII.11; 1993: VII.05, VII.08; 2018: VI.27, VI.29, VIII.06, VIII.08-VIII.09.

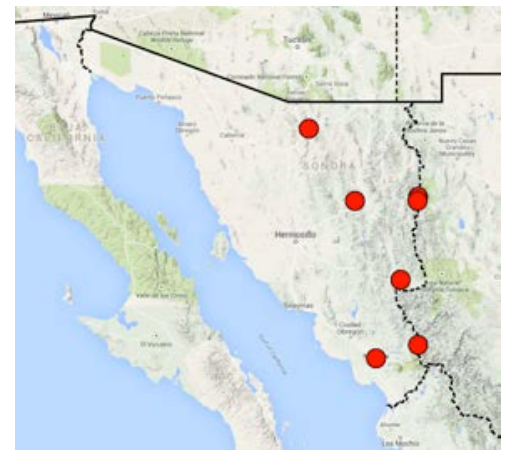

179. Chlaenius tomentosus (Say, 1823)

Number examined: 8.

Municipalities: Cananea, Fronteras, Nácori Chico, Nacozari de García.

Habitat: cow pasture; rocky canyon. [at light]

Vegetation: cypress-maple-alder-sycamore riparian forest; desert grassland; oak woodland; pine-oak forest.

Elevation Range: $1429-1950$ m.

Collection dates: 1983.VII.06; 2013.VII.16; 2015.IX.06; 2016.VII.02; 2018.VIII.09.

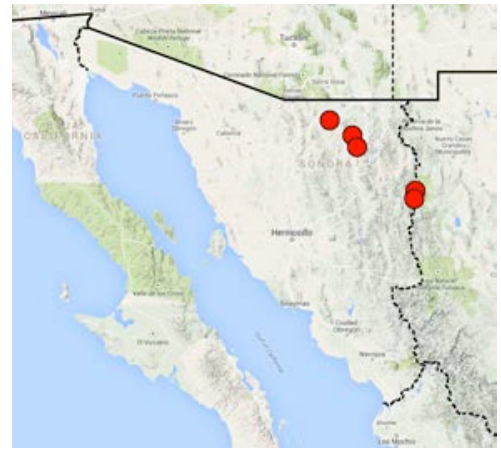

180. Chlaenius tricolor Dejean, 1826

Number examined: 8 .

Municipalities: Agua Prieta, Cananea, Ímuris, Santa Ana. Habitat: urban.

Vegetation: Chihuahuan and Sonoran desertscrub; cottonwood-willow riparian forest; desert grassland.

Elevation Range: $1040-1573$ m.

Collection dates: 1982: VIII.20, VIII.24; 1985.VIII.01; 2013.VIII.04.

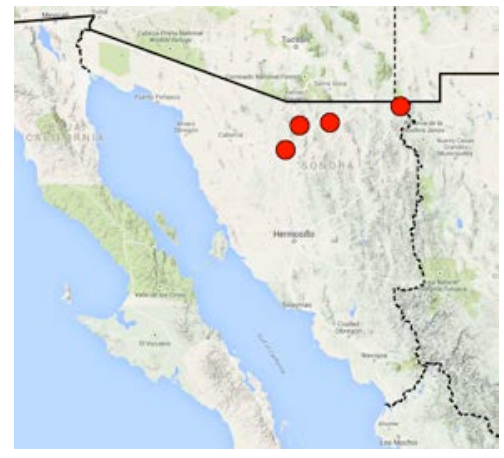

\section{Licinini}

181. Dicaelus suffusus (Casey, 1913)

Number examined: 9 .

Municipalities: Nacozari de García, Nácori Chico.

Habitat: steep north facing rocky slope.

Vegetation: pine-oak forest.

Elevation Range: $1950-2440 \mathrm{~m}$.

Collection dates: 1982: VIII.06-VIII.08; 1983.VII.07; 2015.VIII.10.

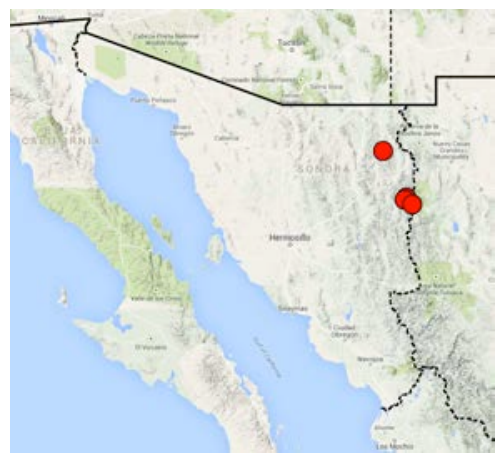




\section{Harpalini \\ 182. Amblygnathus balli, new species}

Fig. 10

http://zoobank.org/4EDCB118-22CA-4E22-BDDED88B816E3548

Etymology: Latinized surname of George E. Ball, colleague, field companion and friend, who passed away on January 12, 2019.

Type material: 2 specimens. Holotype male, labelled: "MEXICO: Sonora/ Municipio de Fronteras,/ Rancho Capulin,/ $23.5 \mathrm{~km}$ (by air) WSW/ Fronteras, SierraBuenos/ Aires, Ajos-Bavispe Res.,"; “30.76167º $109.82361^{\circ} \mathrm{W} /$ cow pasture in oak/ woodland, 1429 m,/ 2.VII.2016, T.R. Van/ Devender, J.D. Palting”; "UASM\#372724" (UNAM). Paratype male, labelled: "USA AZ Cochise Co/ Huachuca Mts, Miller Cyn/ CW Melton 2015.7.6" (PWMC).

Type locality: Mexico, Sonora, Municipio de Fronteras, Rancho Capulín, 23.5 km (by air) WSW Fronteras, Sierra Buenos Aires, $30.76167^{\circ} \mathrm{N} 109.82361^{\circ} \mathrm{W}$.

Diagnosis: This species is readily separated from the only other species of Amblygnathus found in Sonora (Amblygnathus interior) by the rufo-testaceus pronotum.

Description: Member of the Amblygnathus iripennis group (Ball \& Maddison 1987:210), based on characteristics of the male genitalia. Total Length males: 5.64-5.68 mm. Mouthparts, antennae and legs testaceus to dark testaceus. Dorsal surface of head black, pronotum rufo-testaceus and elytra piceous with rufo-testaceus border, dark marking of elytra brightly iridescent (Figure 12A). Ventral surface rufo-testaceus to dark rufo-testaceus, abdomen dark brunneus, last sternite broadly rufo-testaceus apically. Head shiny, isodiametric scupticels very finely impressed; pronotum shiny, no visible micro-lines on the disc, isodiametric scupticels very finely impressed in postero-lateral area; elytra with very fine transverse microsculpture, microlines barely visible, markedly iridescent. Male genitalia (Figure 12A-12B) with apical portion moderately long, with dorsal flange. Lamina long, straight, sharply pointed, spine-like, with long apodeme-like basal extension; nearly straight in dorsal aspect, slightly sinuate in lateral aspect. Internal sac (endophallus) with one large spine, about equal in length to lamina, medial in position, best viewed in right lateral aspect.

Municipality: Fronteras.

Habitat: canyon (Arizona); cow pasture (Sonora).

Vegetation: Madrean oak-juniper woodland (Arizona); oak woodland (Sonora).

Elevation range: 1429 (Sonora) - 1622 (Arizona) $\mathrm{m}$.

Collection dates: 2015.VII.06 (Arizona); 2016.VII.02 (Sonora).

Distribution: This species is only know from two specimens, one from the type locality in Sonora, Mexico, and the second from Miller Canyon, Cochise County in Arizona, United States.

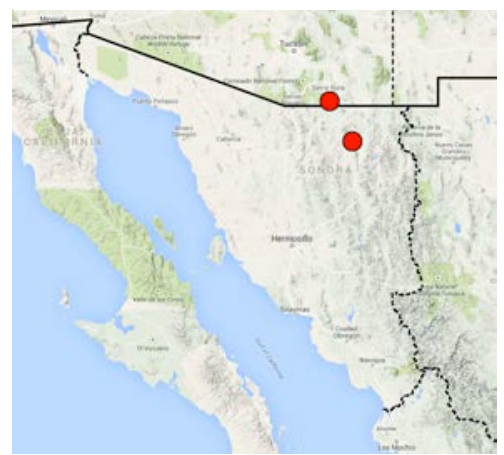

The following change is made to the key to New World species of Amblygnathus in Ball and Maddison (1987:202) to accommodate this new species:

3 (02) Dorsal surface predominately flavous; pronotum uniformly so, or with middle 0.5 black or infuscated; each elytron with basal 0.8 of intervals $1-3$ black Amblygnathus suturalis Putzeys

3' Dorsal surface either uniformly dark, metallic, or head and pronotum rufous, elytra black 04

3" Dorsal surface mainly black, entire pronotum and basal, lateral, apical and sutural margin of elytra rufo-testaceus; disc of elytra iridescent

Amblygnathus balli, new species

183. Amblygnathus interior Ball \& Maddison, 1987

Number examined: 6 .

Municipalities: Álamos, Nácori Chico, Nacozari de Garciá, Yécora.

Habitat: rocky stream canyon and mountainside. [at light]

Vegetation: cypress-maple-alder-sycamore riparian forest; oak woodland; pine-oak forest; tropical deciduous forest.

Elevation range: $1681-1830 \mathrm{~m}$.

Collection dates: 1964.VIII.25; 1993: VII.06-VII.07; 2017.VIII.15; 2018.VI.27.

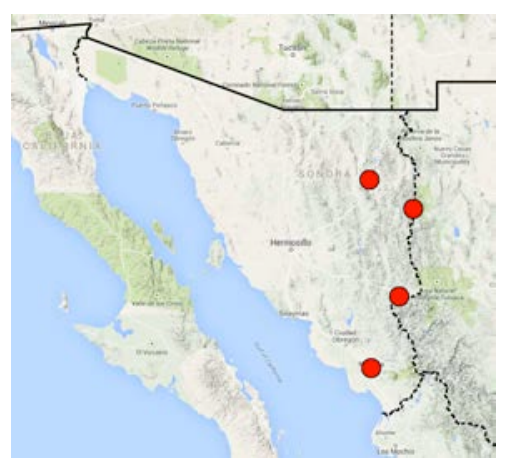




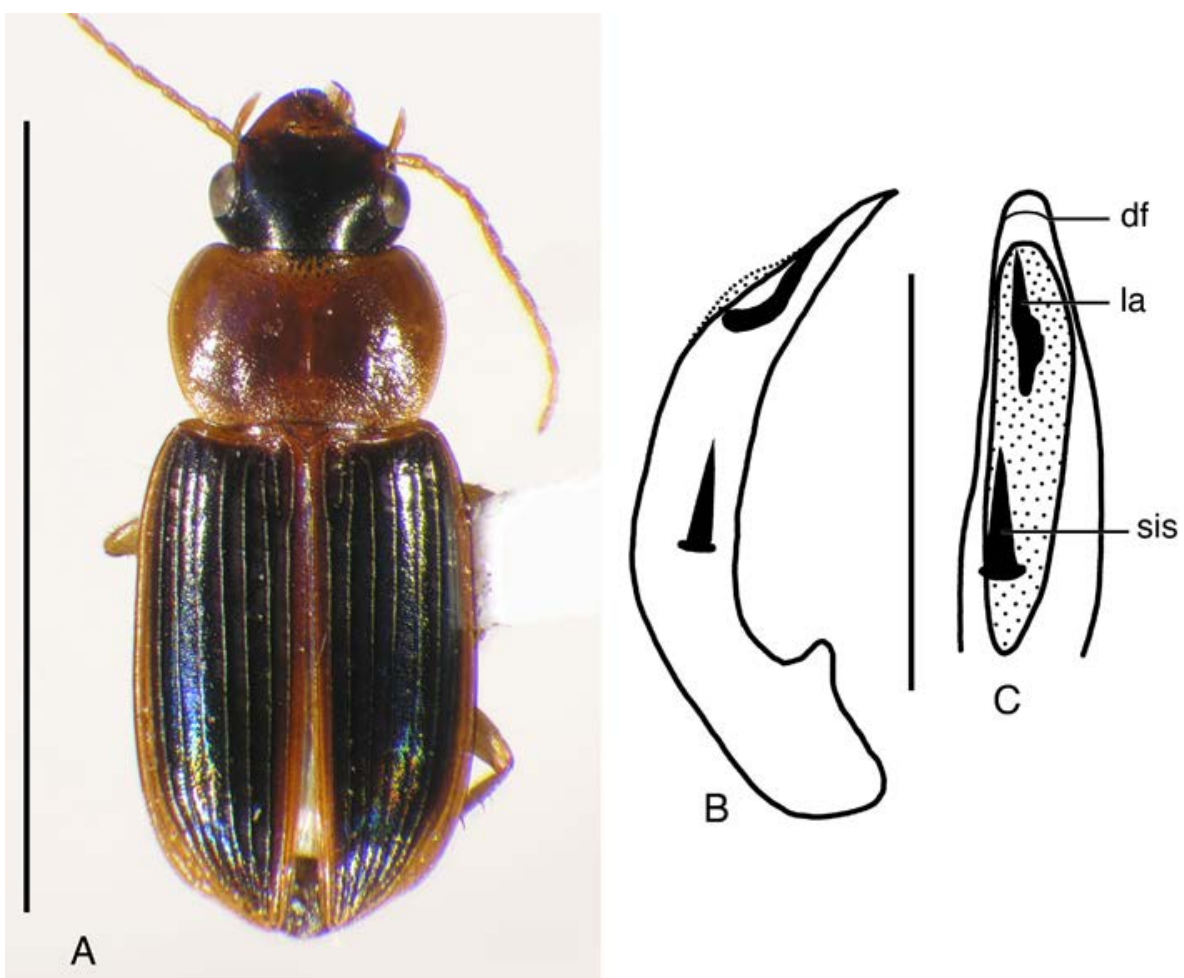

Figure 10. Habitus digital image and line drawings of male genitalia of Amblygnathus balli, new species, holotype. A. Habitus, dorsal aspect. Male genitalia: $\mathbf{B}$. left lateral aspect; $\mathbf{C}$ dorsal aspect. Legend: df dorsal flange; la lamina; sis spine of internal sac. Scale bars: A $6 \mathrm{~mm}$; B, C $1 \mathrm{~mm}$.

184. Amphasia interstitialis (Say, 1823)

Number examined: 1.

Municipality: Ímuris.

Habitat: ?

Vegetation: cottonwood-willow riparian deciduous forest.

Elevation:?

Collection date: 1958.VII.11.

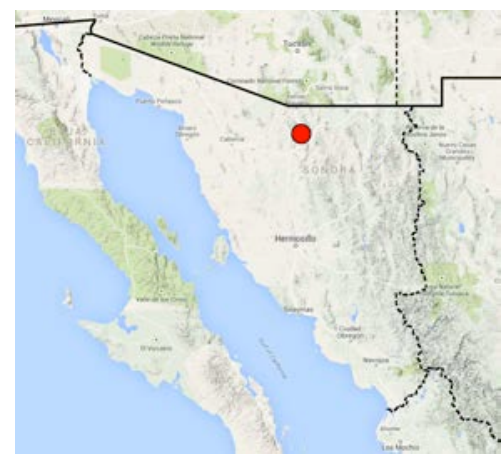

185. Anisodactylus anthracinus (Dejean, 1829)

Number examined: 73.

Municipalities: Bacadéhuachi, Cananea, Fronteras, Huachinera, Ímuris, Mazatán, Moctezuma, Nácori Chico, Nacozari de García, Nogales, Yécora.

Habitat: cow pasture; rocky canyon, slope, and mountainside; urban. [at light]

Vegetation: cottonwood-willow riparian deciduous forest; desert grassland; foothills thornscrub; oak woodland; pine-oak forest; riparian forest, oak woodland.

Elevation Range: $847-2297 \mathrm{~m}$.

Collection dates: 1982: VIII.01, VIII.03, VIII.05, VIII.11; 1983: VII.02, VII.04; 1986.VIII.30; 1990: VI.28-VI.29; 1993: VII.09, VIII.05; 2012.VII.21; 2013.VII.16; 2014.IV.27; 2015: VI.25, VII.12, IX.06; 2016: VII.02, VIII.14; 2017: VII.16, VII.24, VIII.15.

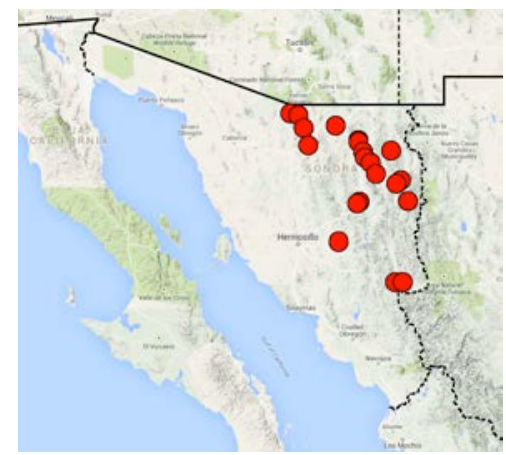

186. Athrostictus sericatus Bates, 1878

Number examined: 12.

Municipalities: Álamos, Carbó, Hermosillo, Santa Ana.

Habitat: [at light]

Vegetation: Sonoran desertscrub; tropical deciduous forest.

Elevation range: $650-707 \mathrm{~m}$.

Collection dates: 1952.VII.19; 1964: VII.28-VII.31, VIII.24, IX.10; 1993.VII.17; 2017.VII 


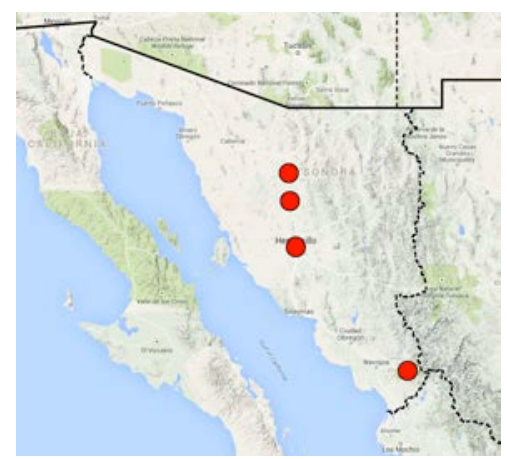

187. Bradycellus nitidus (Dejean, 1829)

Number examined: 5 .

Municipality: Nácori Chico.

Habitat: camp clearing; rocky stream canyon.

Vegetation: cypress-maple-alder-sycamore riparian forest; pine-oak forest.

Elevation: $1648 \mathrm{~m}$.

Collection dates: 2018: VI.27, VIII.06, VIII.09.

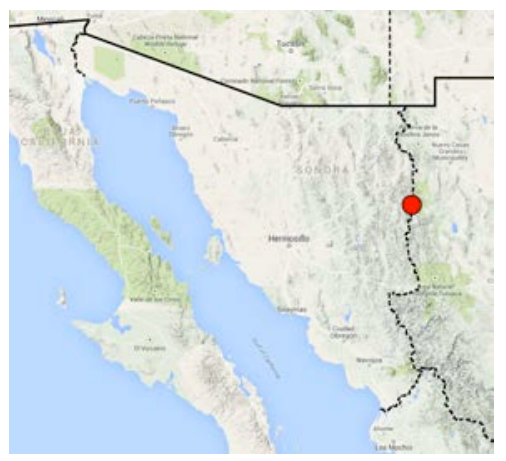

188. Bradycellus rupestris species group sp.

Number examined: 2 .

Municipalities: Ímuris, Nogales.

Habitat: [at light]

Vegetation: cottonwood-willow riparian deciduous forest; desert grassland;

Elevation: ?

Collection dates: 1965: VII.30-VII.31.

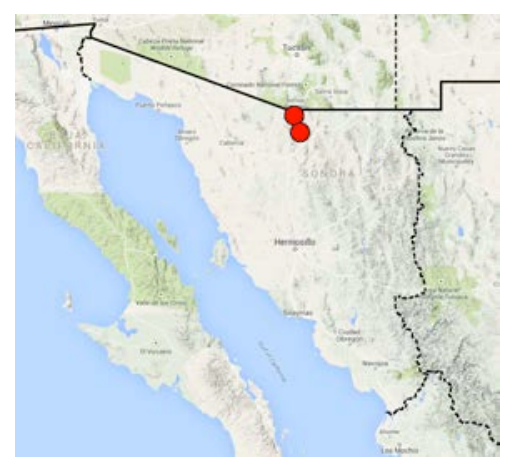

\section{Bradycellus sp. UASM-6}

Number examined: 1 .

Municipality: Ímuris.

Habitat: marsh.

Vegetation: cottonwood-willow riparian deciduous forest.

Elevation: $914 \mathrm{~m}$.

Collection date: 1967.VII.27.

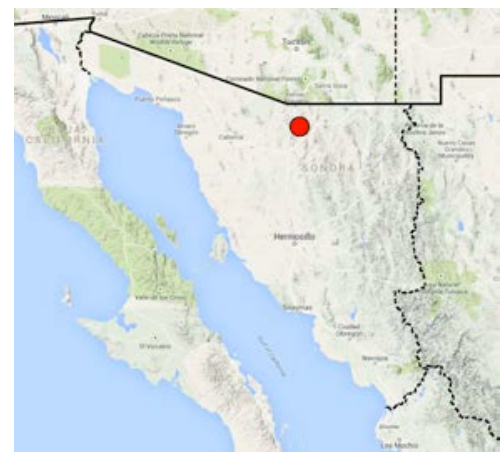

190. Bradycellus spp. (may be more than one species)

Number examined: 190.

Municipalities: Agua Prieta, Fronteras, Guaymas, Huachinera, Ímuris, Moctezuma, Nácori Chico, Sáric, Santa Ana.

Habitat: cow pasture; flood litter, stream margin.

Vegetation: Chihuahuan and Sonoran desertscrub; cottonwood gallery forest; foothills thornscrub; oak woodland; pine-oak forest.

Elevation: $710-2090 \mathrm{~m}$.

Collection dates: 1929.VI.27; 1953: VII.09-VII.16, VIII.15-VIII.20; 1960: VIII.08, VIII.10; 1982: VIII.04, VIII.19-VIII.20, VIII.23-VIII.24; 1983: VII.04-VII.06, VII.11-VII.12; 1986.VIII.30; 2012.VIII.01 2016. VII.02; 2017.IV.23.

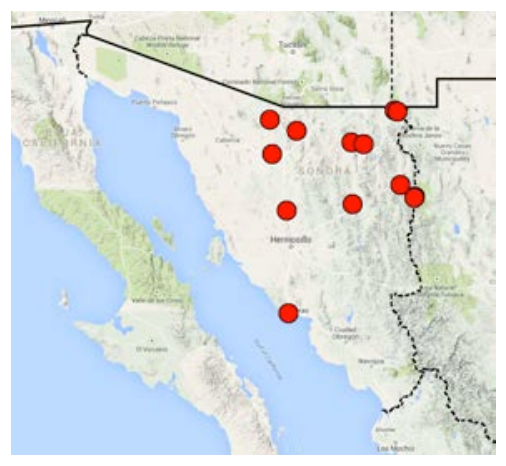

191. Discoderus crassiusculus (Putzeys, 1878)

Number examined: 6.

Municipality: Yécora.

Habitat: ?

Vegetation: pine-oak forest.

Elevation range: 2134 - $2438 \mathrm{~m}$.

Collection dates: 1961: V.20-V.22. 


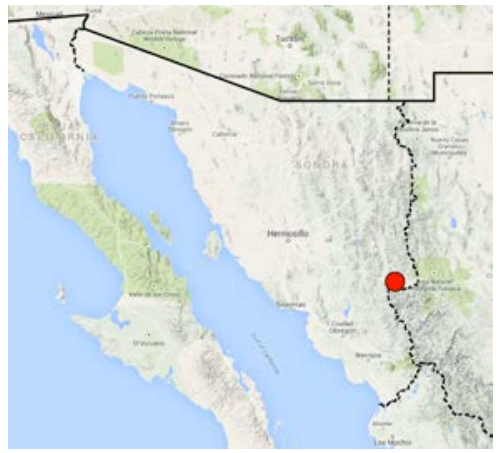

192. Discoderus sp. UASM son-1

Number examined: 1 .

Municipality: Huachinera.

Habitat:?

Vegetation: desert grassland.

Elevation: $1150 \mathrm{~m}$.

Collection date: 1982.VIII.05.

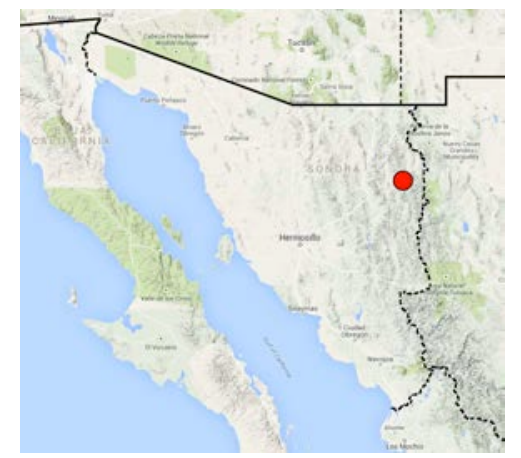

193. Discoderus sp. UASM son-2

Number examined: 8 .

Municipalities: Huachinera, Nácori Chico, Nacozari de Garciá.

Habitat: ?

Vegetation: desert grassland; oak woodland.

Elevation range: $1380-1420 \mathrm{~m}$.

Collection dates: 1982: VIII.03, VIII.11; 1983.VII.04.

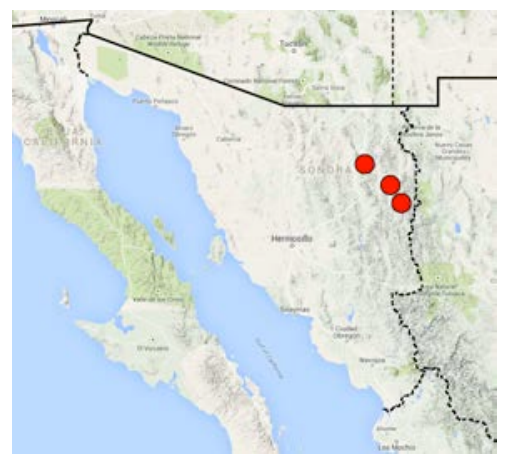

194. Discoderus sp. UASM son-3

Number examined: 314.

Municipalities: Cajeme, Carbó, Empalme, Guaymas, Hermosillo, Huásabas, Huatabampo, Ímuris, La Colorada,
Magdalena de Kino, Nacozari de García, Navojoa, Nogales, Opodepe, Pitiquito, Santa Ana.

Habitat: PEMEX station; urban. [at light]

Vegetation: coastal and foothills thornscrub; cottonwood-willow riparian deciduous forest; desert grassland; Sonoran desertscrub.

Elevation range: $46-1201 \mathrm{~m}$.

Collection dates: 1950: VII.13, VII.21; 1952: VII.19, VII.25-VII.26, VII.29, VII.31; 1953: VII.09-VII.16; 1956.VI.24; 1957.VI.21; 1958.X.08; 1959.VII.22; 1960.VIII.08; 1963.VII.14; 1965: VII.30-VII.31; 1984. VII.02; 1985.VII.31; 1993.VII.17; 2012.VIII.06; 2015: VII.12, VIII.13; 2016.V.05, 2017: II.27, VII.21-VII.23.

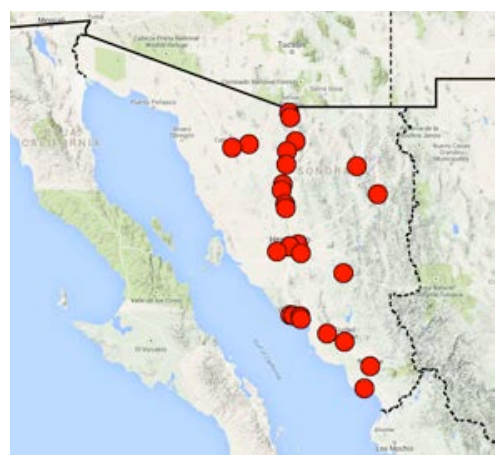

195. Discoderus sp. UASM-8

Number examined: 230 .

Municipalities: Agua Prieta, Álamos, Arizpe, Bacadehuachi, Bacoachi, Banamichi, Bavispe, Cananea, Cucurpe, Cumpas, Divisaderos, Fronteras, Granados, Hermosillo, Huachinera, Ímuris, Magdalena de Kino, Mazatán, Moctezuma, Nacozari de Garciá, Nogales, San Felipe de Jesús, Santa Ana, Santa Cruz.

Habitat: flood litter; pond margin; rocky arroyo, mountainside, ridge top, slopes, and strean canyon; urban. [at light]

Vegetation: Chihuahuan and Sonoran desertscrub; cottonwood-willow riparian forest; desert grassland; foothills thornscrub; oak woodland; pine-oak forest; tropical deciduous forest.

Elevation range: $212-2441 \mathrm{~m}$.

Collection dates: 1965: VII.31, IX.11, IX.16; 1968.VI.18; 1982: VIII.02-VIII.03, VIII.05, VIII.11; 1986: VI.28, VIII.30; 1993.VII.05; 2012: VIII.09, VIII.13, IX.05; 2013: VIII.12, VIII.23, IX.09; 2014.VIII.25; 2015: VIII.10, VIII.15, IX.06, IX.14; 2016: III.04, III.29, IV.01, IV.07, IV.16, VIII.24, XI.21-XI.22; 2017: IV.21, VII.16, VII.21, VII23, VIII.13-VIII.16; 2018: III.19III.20; 2019: IV.25, VII.06, VIII.04-VIII.05, VIII.09, VIII.16, IX.14, IX.23, IX.25-IX.26. 


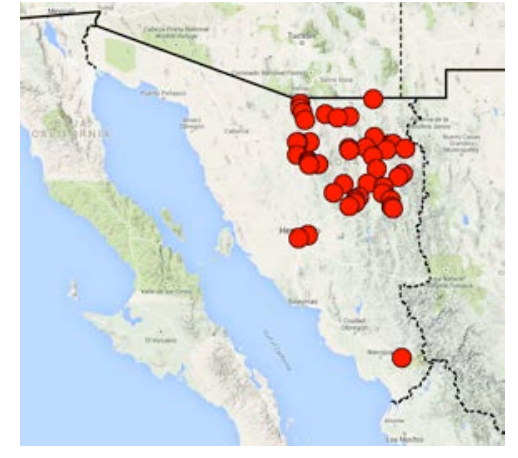

196. Discoderus sp. UASM-11

Number examined: 56.

Municipalities: Bacanora, Huachinera, Moctezuma.

Habitat: rocky slope.

Vegetation: desert grassland; foothills thornscrub; oak woodland; riparian tropical scrub.

Elevation range: $944-1387 \mathrm{~m}$.

Collection dates: 1982: VIII.01, VIII.05; 1993.VII.05; 2014.VIII.02; 2019.VIII.09.

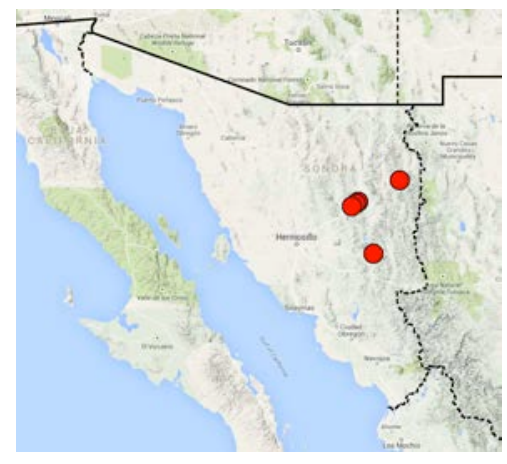

197. Discoderus sp. UASM-13

Number examined: 66 .

Municipalities: Agua Prieta, Cananea, Granados, Ímuris, Naco, Nogales.

Habitat: urban. [at light]

Vegetation: Chihuahuan desertscrub; cottonwood-willow riparian deciduous forest; desert grassland; foothills thornscrub.

Elevation range: $858-1573 \mathrm{~m}$.

Collection dates: 1949.VIII.15; 1965.VII.30; 2013: VIII.04, VIII.23; 2015: VII.12, VIII.08, IX.06; 2016: III.29, VIII.24; 2017.IX.02; 2018.VIII.13.

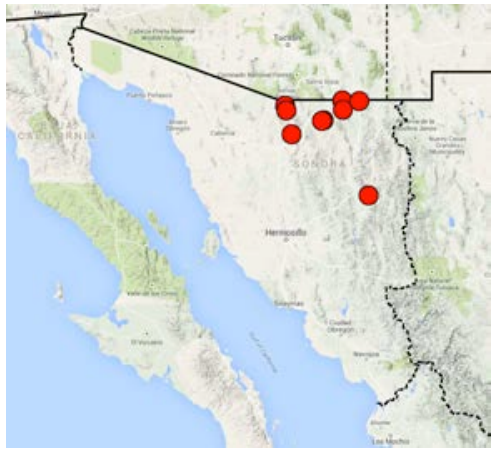

198. Discoderus sp. UASM-16

Number examined: 83.

Municipalities: Agua Prieta, Cananea, Fronteras, Ímuris, Magdalena de Kino, Naco, Nacozari de García, Nogales, Santa Ana.

Habitat: urban. [at light]

Vegetation: Chihuahuan and Sonoran desertscrub; cottonwood-willow riparian deciduous forest; desert grassland; oak woodland.

Elevation range: $742-1573 \mathrm{~m}$

Collection dates: 1964.IX.06; 1965: VII.30, IX.04, IX.11; 2012.VIII.01; 2013: VIII.04, VIII.23; 2016.VIII.24; 2017.IX.02.

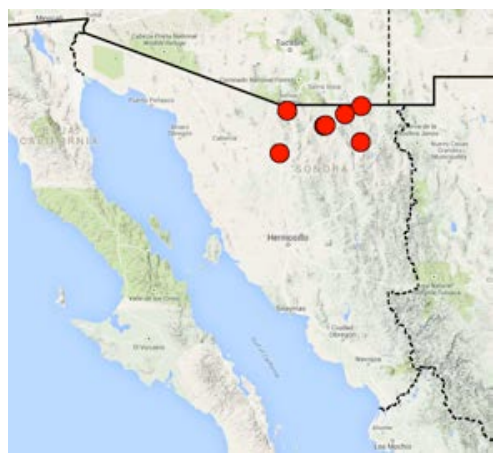

199. Harpalus caliginosus (Fabricius, 1775)

Number examined: 10.

Municipalities: Bacoachi, Cananea, Ímuris, Nácori Chico, Yécora.

Habitat: arroyo; meadow; stream canyon.

Vegetation: cottonwood-willow riparian deciduous forest; desert grassland; oak woodland; pine-oak forest.

Elevation range: $1204-1950 \mathrm{~m}$.

Collection dates: 1965.VII.31; 1983.VII.06; 1984.VIII.25; 1990.VIII.11; 2016.VIII.25; 2018.VIII.07. 


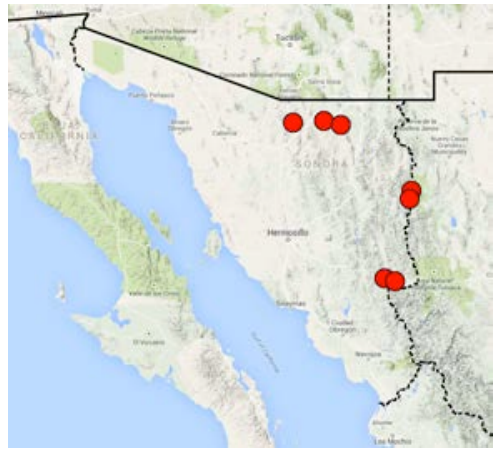

200. Harpalus desertus LeConte, 1859

Number examined: 15.

Municipalities: Cananea, Nácori Chico, Nacozari de García.

Habitat: shallow rocky arroyo, stream margin.

Vegetation: desert grassland; oak woodland; pine-oak forest.

Elevation rane: $1521-2278 \mathrm{~m}$.

Collection dates: 1982: VIII.06-VIII.07; 2015.VIII.11; 2016.VIII.24.

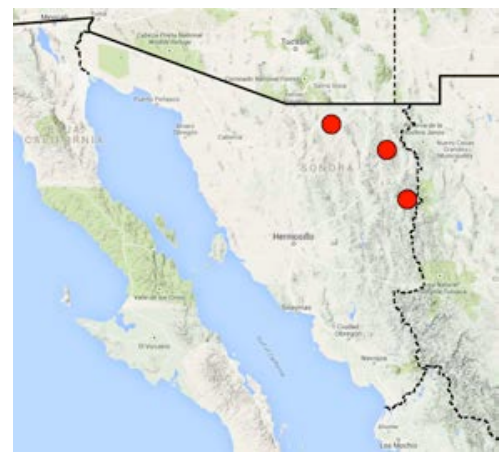

201. Harpalus fraternus LeConte, 1852

Number examined: 1

Municipality: Nogales.

Habitat: ?

Vegetation: desert grassland.

Elevation: $1081 \mathrm{~m}$.

Collection date: 2013.VIII.09.

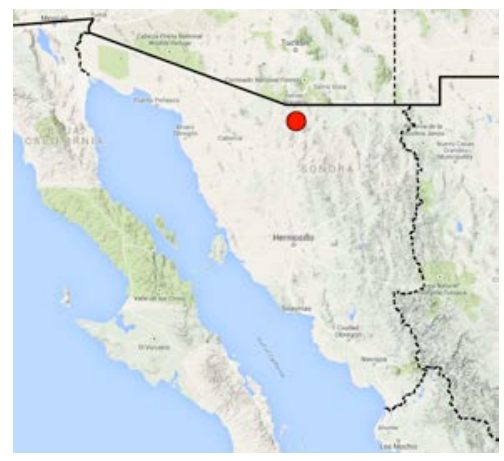

202. Harpalus pensylvanicus (DeGeer, 1774)

Number examined: 104.

Municipalities: Aconchi, Agua Prieta, Cananea, Fronteras, Ímuris, Moctezuma, Nácori Chico, Nogales, Yécora.

Habitat: camp clearing; pasture; rocky stream canyon; stream margin. [at light]

Vegetation: Chihuahuan desertscrub; cottonwood-willow riparian forest; cypress-maple-alder-sycamore desert grassland; foothills thornscrub; pine-oak forest; riparian forest.

Elevation range: $606-1950 \mathrm{~m}$.

Collection dates: 1965: VII.30-VII.31; 1982: VIII.01, VIII.06-VIII.07; 1983.VII.05; 1990: VI.30-VII.03, VIII.11; 2012.VIII.01; 2015.IX.06; 2018: VI.27, VIII.05-VIII.09; 2019.VIII.15.

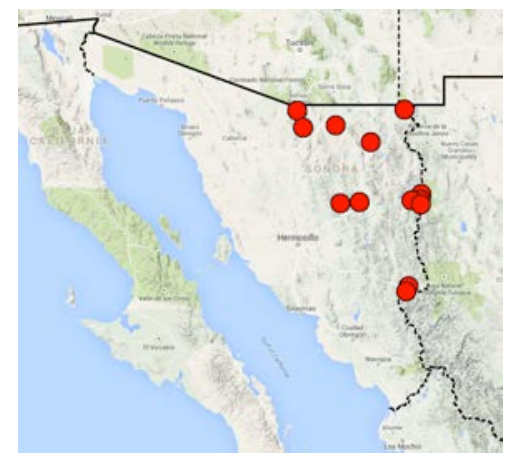

203. Notiobia brevicollis (Chaudoir, 1837)

Number examined: 52 .

Municipalities: Agua Prieta, Álamos, Bacadéhuachi, Cananea, Huachinera, Ímuris, Naco, Nácori Chico, Nacozari de García, Nogales, Yécora.

Habitat: camp clearing; pasture; rocky stream canyon and ridge top; urban. [at light]

Vegetation: Chihuahuan desertscrub; cottonwood-willow riparian forest; cypress-maple-alder-sycamore riparian forest; desert grassland; foothills thornscrub; oak woodland; pine-oak forest; tropical deciduous forest.

Elevation Range: $1204-2261 \mathrm{~m}$.

Collection dates: 1965: VII.30-VII.31; 1982: VIII.03, VIII.06-VIII.07, VIII.19-VIII.20; 1989: VII.11-VII.13; 1990: VII.02-VII.03, VIII.11; 1993.VII.08; 2013: VII.16, VIII.04; 2015: VIII.12, IX.06; 2017.IX.02; 2018: VI.27, VIII.06, VIII.09.

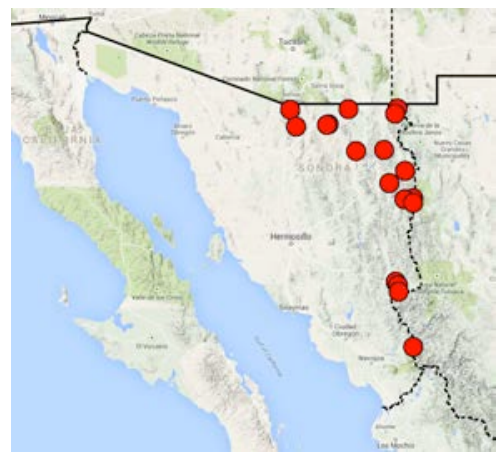


Number examined: 12.

204. Notiobia cyanippa (Bates, 1882)

Municipalities: Álamos, Fronteras, Nácori Chico, Nacozari de García.

Habitat: cow pasture; gravel; rocky arroyo; rocky canyon; rocky mountainside.

Vegetation: Chihuahuan desertscrub; foothills thornscrub; oak woodland; sycamore riparian forest; tropical deciduous forest.

Elevation range: $950-1595 \mathrm{~m}$.

Collection dates: 1935.VII.07; 1982.VIII.09; 1983.VII.04; 2013.VII.16; 2016: VII.02, VIII.15; 2017: VII.15, VIII.14-VIII.15.

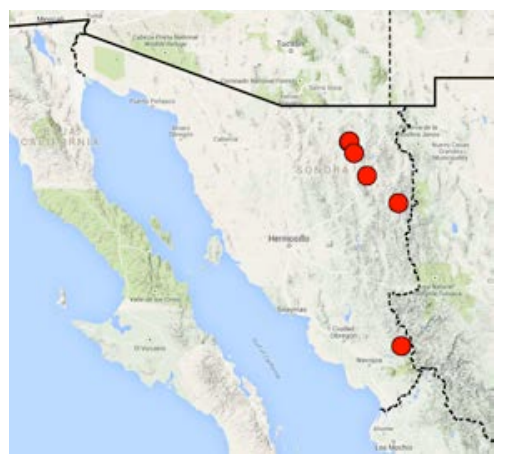

205 Notiobia mexicana Dejean, 1829

Number examined: 127.

Municipalities: Aconchi, Álamos, Bacadéhuachi, Bacoachi, Cananea; Fronteras, Huachinera, Magdalena de Kino, Moctezuma, Nácori Chico, Nacozari de García, Yécora.

Habitat: cow pasture; rocky arroyo, stream canyon, slope, mountainside, and ridge top.

Vegetation: Chihuahuan and Sonoran desertscrub; cypress-maple-alder-sycamore riparian forest; desert grassland; foothills thornscrub; oak woodland; pine-oak forest; tropical deciduous forest.

Elevation Range: 1000 - $2467 \mathrm{~m}$.

Collection dates: 1982: VIII.03-VIII.07, VIII.11; 1983: VII.04-VII.07; 1989: VII.11-VII.13; 1990: VI.28VII.03, VIII.11; 1993: VII.05-VII.07; 2012.VIII.09; 2013: VII.03, VII.16, IX.03; 2014.IX.20; 2015: VI.25, VIII.10, VIII.12, IX.06, IX.13-IX.14; 2016: VII.02, VIII.14-VIII.15; 2017.VIII.15; 2018: VI.27, VI.29; 2019: IX.24-IX.25.

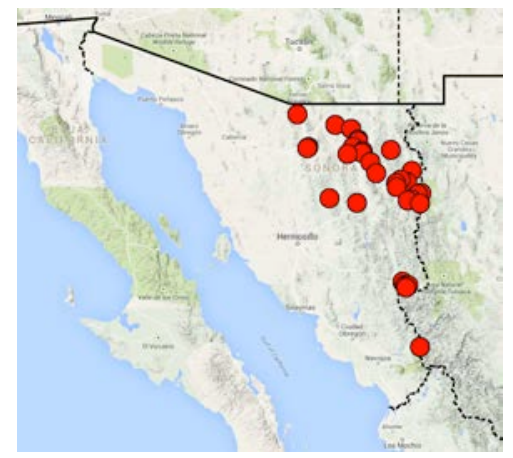

206. Notiobia purpurascens (Bates, 1882)

Number examined: 22.

Municipalities: Agua Prieta, Ímuris, Navojoa.

Habitat: [at light]

Vegetation: Chihuahuan desertscrub; cottonwood-willow riparian forest; coastal thornscrub.

Elevation Range: $1040-1350 \mathrm{~m}$.

Collection dates: 1972.VII.18; 1982: VIII.20, VIII.24.

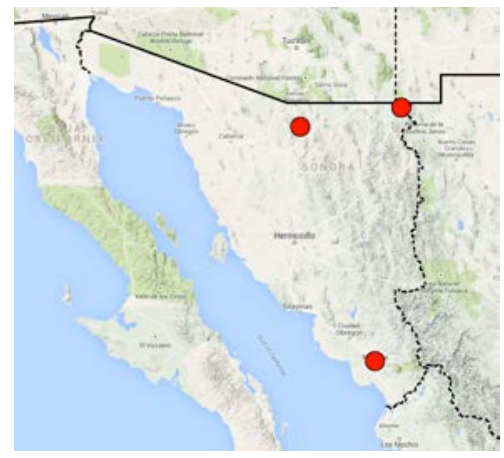

207. Notiobia schlingeri Noonan, 1973

Number examined: 17.

Municipality: Nácori Chico.

Habitat: camp clearing. [at light]

Vegetation: cypress-maple-alder-sycamore riparian forest; pine-oak forest.

Elevation: $1648 \mathrm{~m}$.

Collection dates: 2018: VIII.06-VIII.09.

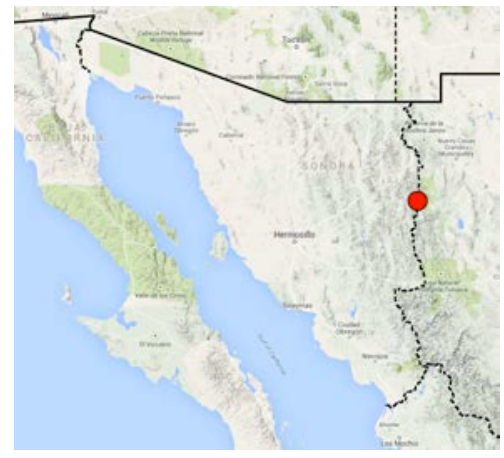

208. Notiobia terminata (Say, 1823)

Number examined: 3 .

Municipalities: Álamos, Nacozari de García. 
Habitat: large flat area near ridge top. [at light] Vegetation: pine-oak forest; tropical deciduous forest. Elevation: $2261 \mathrm{~m}$.

Collection dates: 1964.VIII.24; 2015.VIII.13.

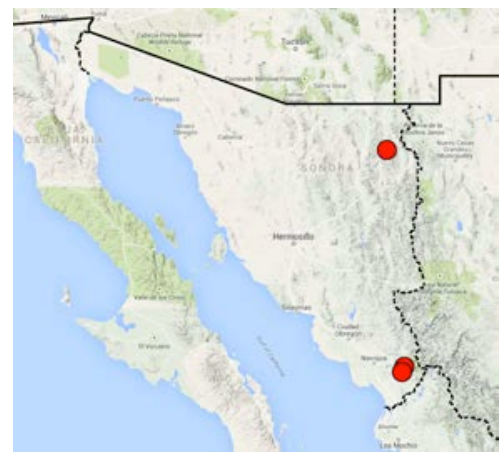

209. Pelmatellus stenolophoides Bates, 1882

Number examined: 1 .

Municipality: Nácori Chico.

Habitat: camp clearing

Vegetation: cypress-maple-alder-sycamore riparian forest; pine-oak forest.

Elevation: $1648 \mathrm{~m}$.

Collection date: 2018. VIII.06.

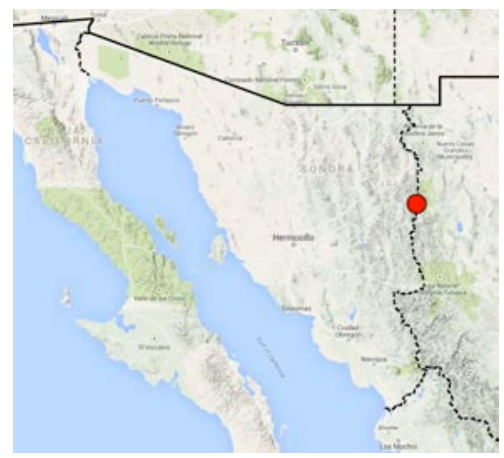

210. Pogonodaptus mexicanus (Bates, 1878)

Number examined: 2 .

Municipalities: Guaymas, Navojoa.

Habitat: [at light]

Vegetation: coastal thornscrub.

Elevation: ?

Collection dates: 1964.VIII.25; 1965.VIII.01.

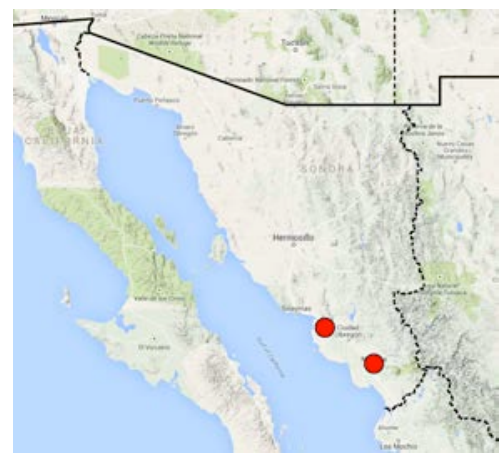

211. Polpochila erro (LeConte, 1854)

Number examined: 61 .

Municipios: Agua Prieta, Álamos, Carbó, Benjamín Hill, Hermosillo, Huatabampo, Magdalena de Kino, Nácori Chico, Opodepe, Pitiquito, Santa Ana.

Habitat: rocky stream canyon; PEMEX station. [at light]

Vegetation: Chihuahuan and Sonoran desertscrub; oak woodland; pine-oak forest; tropical deciduous forest.

Elevation range: $741-1605 \mathrm{~m}$.

Collection dates: 1950.VIII.10; 1953: VII.09-VII.16, VIII.20, IX.26; 1957: VII.28-VII.29; 1963.VII.15; 1965.VII.31; 1967.VII.27; 1980.IX.16; 1982: VIII.19VIII.20, VIII.23; 1985.VII.31; 1987: VII-30-VII.31; 2012.IX.10; 2013.VIII.23; 2017.VII.23; 2018: VI.29, VIII.13.

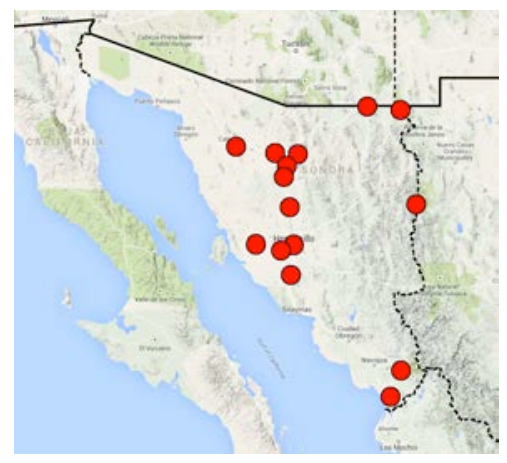

212. Selenophorus aeneopiceus Casey, 1884

Number examined: 51.

Municipalities: Agua Prieta, Banámichi, Cananea, Carbó, Fronteras, Ímuris, Magdalena de Kino, Naco, Nacozari de García, Nogales.

Habitat: cow pasture; PEMEX station; rocky mountainside; small limestone hill; urban. [at lights on building at night]

Vegetation: Chihuahuan and Sonoran desertscrub; cottonwood-willow riparian deciduous forest; desert grassland; foothills thornscrub; oak woodland.

Elevation range: $742-2297 \mathrm{~m}$.

Collection dates: 1968. VI.19; 2012: VII.13, VIII.09; 2013: VII.05, VIII.23; 2015: VI.25, VII.12, VIII.13; 2016: II.09, VII.02; 2017: VII.16, VIII.15.

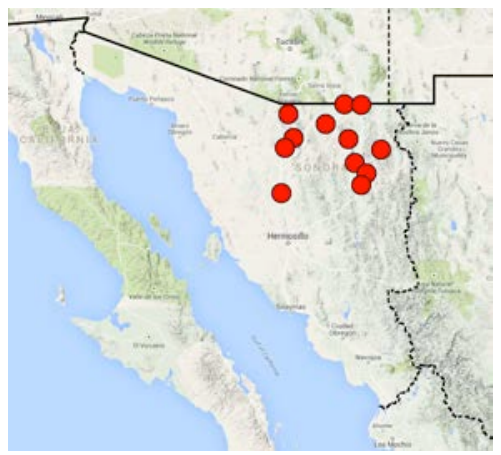


213. Selenophorus aequinoctialis Dejean, 1829

Number examined: 613.

Municipalities: Agua Prieta, Álamos, Banámichi, Benjamín Hill, Cananea, Carbó, Empalme, Fronteras, Granados, Guaymas, Hermosillo, Ímuris, La Colorada, Magdalena de Kino, Mazatán, Moctezuma, Nacozari de García, Navojoa, Nogales, Opodepe, Pitiquito, Rayón, San Felipe de Jesús, Santa Ana.

Habitat: cow pasture; lights at building at night; PEMEX station; rocky slope; urban. [at light]

Vegetation: Chihuahuan and Sonoran desertscrub; cottonwood-willow riparian deciduous forest; desert grassland; foothills thornscrub; oak woodland; tropical deciduous forest.

Elevation range: $33-1573 \mathrm{~m}$.

Collection dates: 1950.VII.13; 1953: VIII.09-VIII.16, VIII.21-VIII.30; 1957.VIII.16; 1960: VIII.09, VIII.10, VIII.12; 1963: VIII.03, VIII.06-VIII.07; 1964: VIII.08, VIII.23-VIII-25, IX.03, IX.06; 1965: VII.15, VII.30VII.31; 1967.VII.27; 1969.VIII.19; 1971.VIII.16; 1980. IX.16; 1982: VIII.02, VIII.04, VIII.23; 1983: VII.11VII.12; 1984.VII.04; 1985.VII.31; 1986: VIII.05, VIII.29; 1987: VII.30-VII.31; 1989: VII.08, VII.11VII.13; 1993: VII.04, VII.14. 2012: VII.12-VII.13, VIII.01, VIII.06, VIII.08-VIII.09, IX.05; 2013: VII.05, VIII.04, VIII.09, VIII.23; 2014: VIII.02, VIII.29; 2015: VI.27, VII.12, VIII.08, VIII.13; 2016.VII.02; 2017: V.23, VII.16, VII.21-VII.23; 2018: VIII.11, VIII.13; 2019: VIII.05, VII.25.

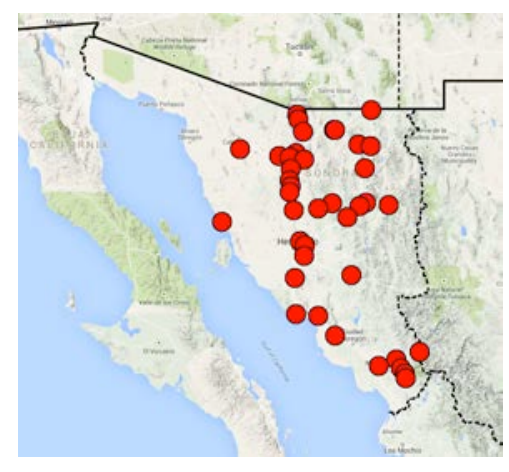

214. Selenophorus balli Messer \& Raber, 2021

Number examined: 234.

Municipalities: Álamos, Hermosillo, La Colorada, Navojoa.

Habitat: [at light]

Vegetation: coastal thornscrub; Sonoran desertscrub; tropical deciduous forest.

Elevation range: $229-518 \mathrm{~m}$.

Collection dates: 1960.VIII.12; 1964: VII.24-VII.25, IX.10; 1967.VII.27; 1987: VII.30-VII.31; 2012.VIII.06.

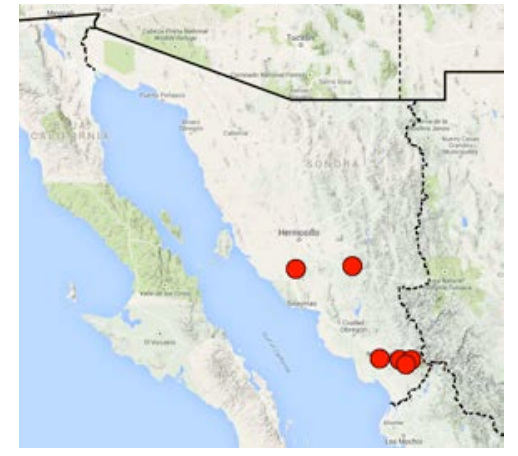

215. Selenophorus concinnus Schaeffer, 1910

Number examined: 314 .

Municipalities: Álamos, Aconchi, Cananea, Carbó, Fronteras, Hermosillo, Huchineras, Ímuris, Mazatán, Moctezuma, Nácori Chico, Nacozari de García, Nogales, Navojoa, Ónavas, Pitiquito, Yécora.

Habitat: cow pasture; rocky stream canyon, slopes, and mountainside; urban. [at light]

Vegetation: Chihuahuan and Sonoran desertscrub; cottonwood-willow riparian deciduous forest; desert grassland; foothills thornscrub; oak woodland; pine-oak forest; riparian forest; riparian tropical scrub; sycamore riparian deciduous forest; tropical deciduous forest.

Elevation: $858-2297 \mathrm{~m}$.

Collection dates: 1953: VII.08-VII.16; 1960: VIII.08, VIII.12; 1964: VIII.08, VIII.24-VIII.25; 1967.VII.22; 1981.VI.26; 1982: VIII.01, VIII.04, VIII.09; 1983: VII.03-VII.04, VII.06, VII.11-VII.12; 1987: VII.26VII.27; 1989.VII.08; 1990: VI.28-VI.29, VII.02-VII.03; 1993.VII.04; 2013.VII.02, VII.16; 2014: VII.28, VIII.24; 2015: VI.25, VI.27, VII.12, VIII.08; 2016: VII.02, VIII.14; 2017: VII.24, VIII.14-VIII.15.

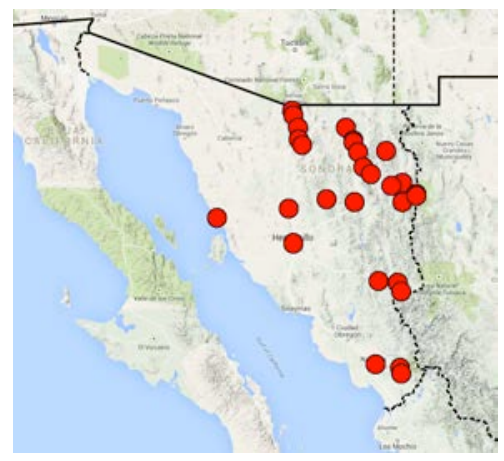

216. Selenophorus dispar Bates, 1891

Number examined: 2 .

Municipalities: Carbó, Hermosillo.

Habitat: ?

Vegetation: Sonoran desertscrub.

Elevation: ?

Collection dates: 1965.VII.31; 1967.VII.27. 


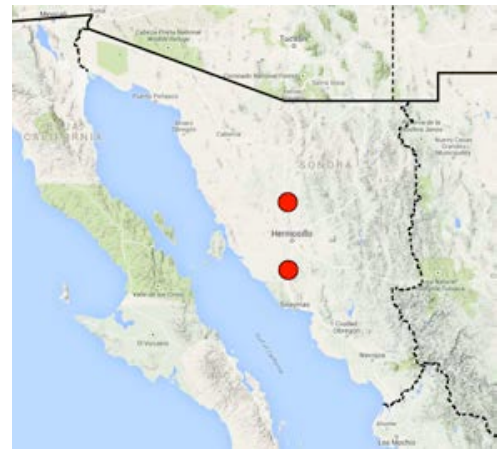

217. Selenophorus fatuus (LeConte, 1863)

Number examined: 212.

Municipalities: Álamos, Bacanora, Carbó, Empalme, Granados, Hermosillo, Mazatán, Moctezuma, Nacozari de García, Nogales, Navojoa, Pitiquito.

Habitat: rocky slope; PEMEX station; urban. [at light]

Vegetation: coastal and foothills thornscrub; desert grassland; oak woodland; Sonoran desertscrub; tropical deciduous forest.

Elevation range: 229 - $1387 \mathrm{~m}$.

Collection dates: 1953: VIII.20-VIII.31; 1954.VIII.24; 1960: VIII.08, VIII.12; 1964: VIII.24-VIII.25, IX.10; 1965.VII.31; 1967.VII.27; 1983: VII.11-VII.12; 2014.08.02; 2015: VII.12, VIII.13; 2017: VII.21VII.22; 2018.VIII.11.

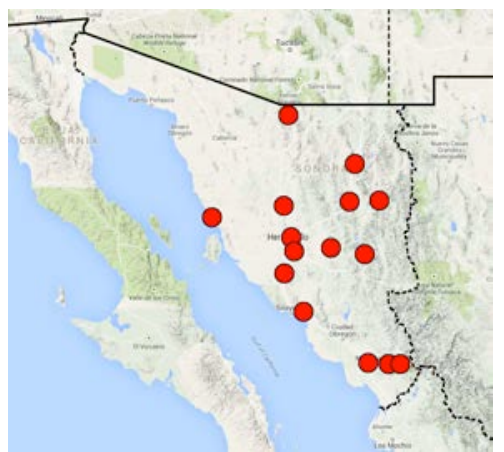

218. Selenophorus pedicularis Dejean, 1829

Number examined: 15.

Municipalities: Álamos, Hermosillo, Saric.

Habitat: ?

Vegetation: Sonoran desertscrub tropical deciduous forest. Elevation: ?

Collection dates: 1929.VI.27; 1953: VII.09-VII.16; 1973.X.11.

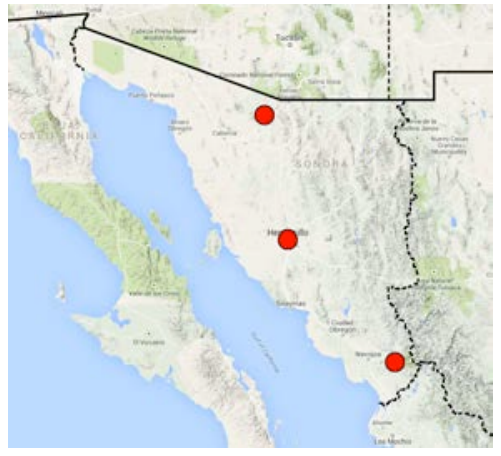

219. Selenophorus pumilus Messer \& Raber, 2021

Number examined: 17.

Municipalities: Álamos, Hermosillo, Navojoa.

Habitat: ?

Vegetation: coastal thornscrub; Sonoran desertscrub; tropical deciduous forest.

Elevation: ?

Collection dates: 1953: VII.09-VII.16; 1956.VI.24; 1960. VIII.12.

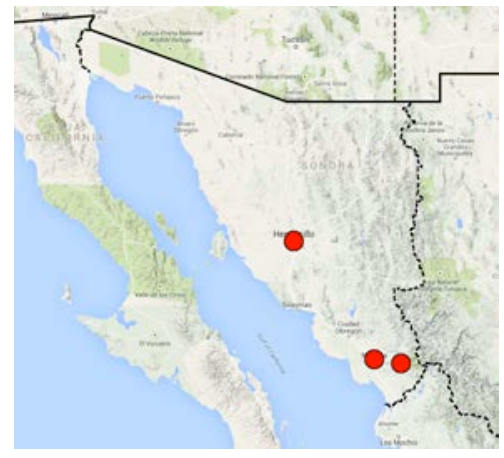

220. Selenophorus pyritosus Dejean, 1829

Number examined: 67.

Municipalities: Álamos, Navojoa.

Habitat: [at light]

Vegetation: coastal thornscrub; tropical deciduous forest. Elevation: ?

Collection dates: 1962.VI.24; 1964.VIII.25; 1989.VII.08.

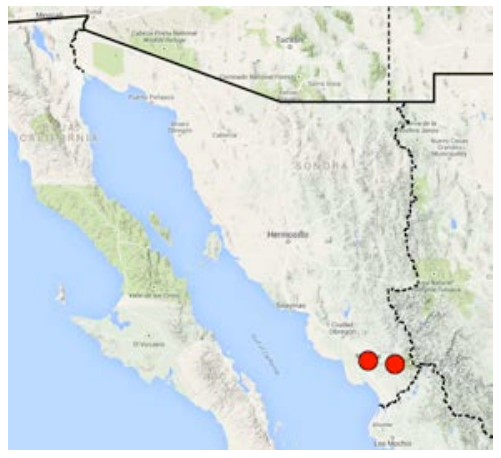


Number examined: 8 .

Municipality: Álamos.

Habitat: [at light]

Vegetation: tropical deciduous forest.

Elevation range: ?

Collection dates: 1960.VIII.12; 1964: VIII.24, IX.03.

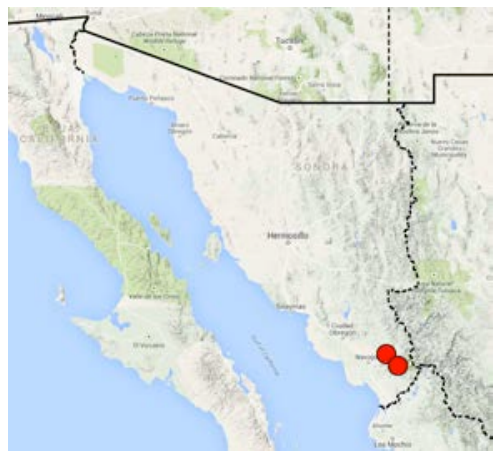

222. Selenophorus semirufus Bates, 1882

Number examined: 5 .

Municipalities: Cajeme, Hermosillo, Naco.

Habitat: ?

Vegetation: coastal thornscrub; desert grassland; Sonoran desertscrub.

Elevation: $1416 \mathrm{~m}$.

Collection dates: 1953: VII.09-VII.16; 1960.VIII.11; 2017.IX.02.

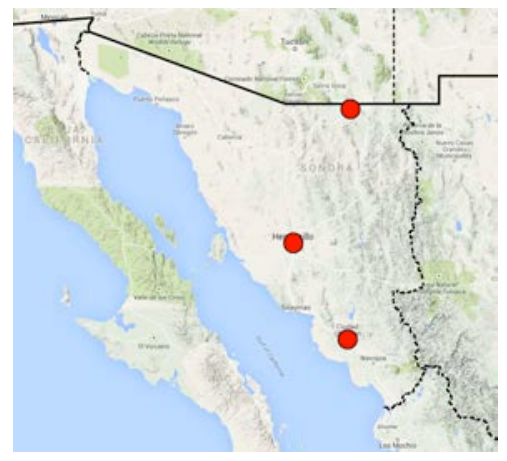

223. Selenophorus seriatoporus Putzeys, 1878

Number examined: 23

Municipalities: Aconchi, Álamos, Nácori Chico, Ónavas.

Habitat: rocky canyon and mountainside. [at light]

Vegetation: foothills thornscrub; oak woodland; sycamore riparian deciduous forest; tropical deciduous forest.

Elevation range: 917 - $1681 \mathrm{~m}$.

Collection dates: 1964: VII.24, VIII.08, VIII.24; 1983.

VII.04; 1987: VII.26-VII.27; 1989: VII.11-VII.13; 2013.VII.02; 2017 . VIII.15.

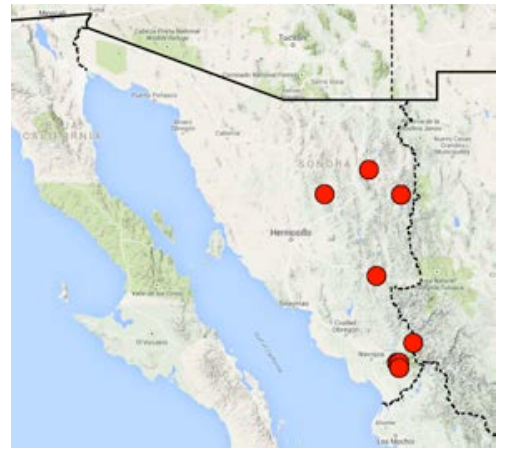

224. Selenophorus sinuaticollis Notman, 1922 Number examined: 31 .

Municipalities: Carbó, Navojoa, Pitiquito, Santa Ana.

Habitat: ?

Vegetation: coastal thornscrub; Sonoran desertscrub.

Elevation range: $650-850 \mathrm{~m}$.

Collection date: 1956.VI.24; 1982.VIII.23; 1985.

VII.31; 1993: VII.14, VII.17; 2017.VII.23.

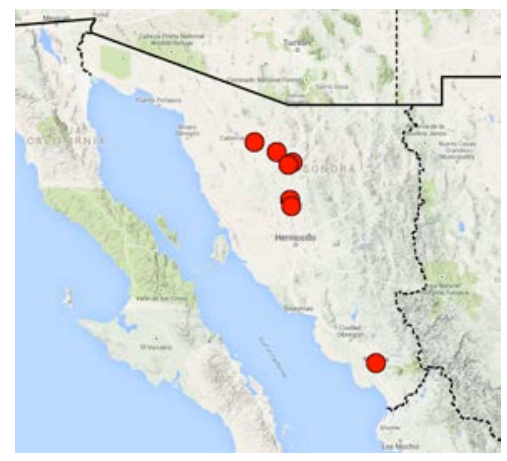

225. Selenophorus striatopunctatus Putzeys, 1878

Number examined: 2.

Municipalities: Navojoa, Soyopa.

Habitat: ?

Vegetation: coastal and foothills thornscrub.

Elevation: $278 \mathrm{~m}$.

Collection dates: 1964.VIII.25; 1965.VII.11.

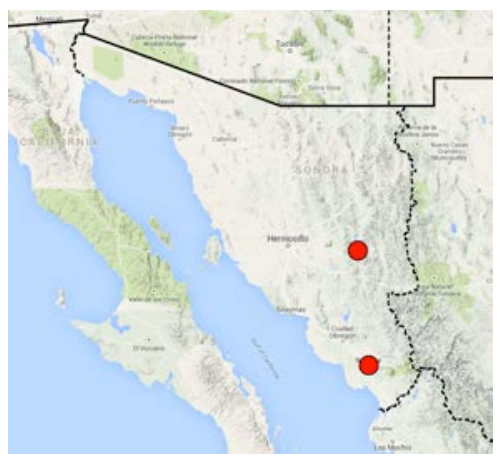


226. Selenophorus hylacis group sp. UASM-12

Number examined: 18.

Municipalities: Álamos, Navojoa.

Habitat: [at light]

Vegetation: coastal thornscrub; tropical deciduous forest.

Elevation: ?

Collection dates: 1964: VIII.24-VIII.25.

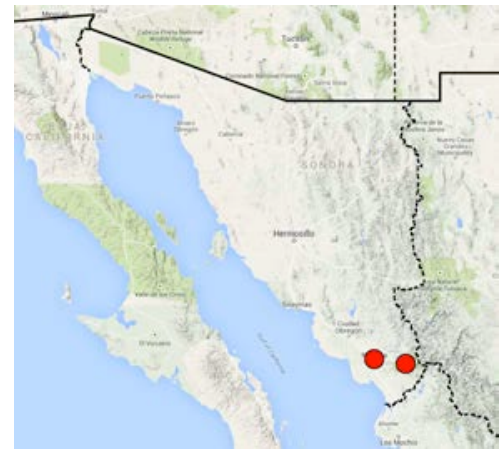

227. Selenophorus hylacis group sp. UASM-14

Number examined: 2.

Municipalities: La Colorada, Rosario de Tesopaco.

\section{Habitat: ?}

Vegetation: foothills thornscrub; Sonoran desertscrub.

Elevation: 413 m.

Collection dates: 1971.IX.05; 2012.VIII.06.

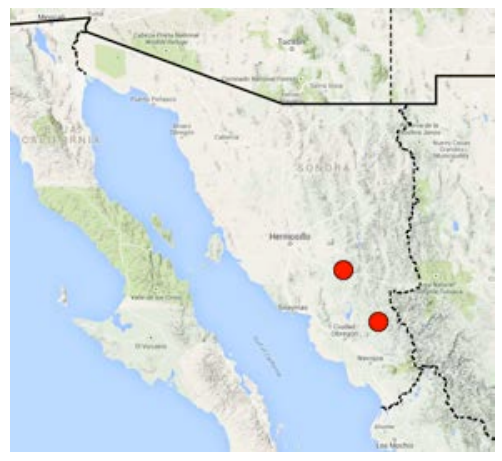

228. Selenophorus suavis group sp. UASM-6

Number examined: 1.

Municipality: Nogales.

Habitat: ?

Vegetation: desert grassland.

Elevation: $1159 \mathrm{~m}$.

Collection date: 2015.VI.27.

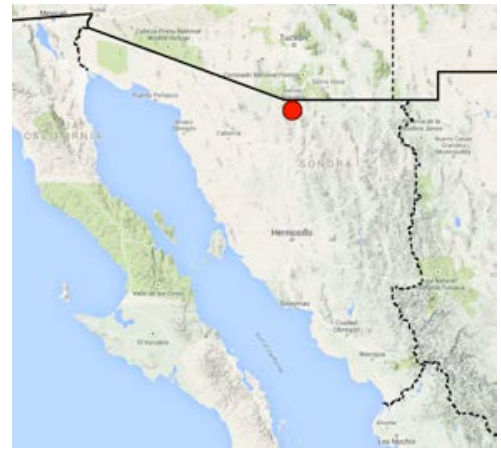

229. Selenophorus (Hemisopalus) sp. 1

Number examined: 43.

Municipality: Álamos.

Habitat: [at light]

Vegetation: tropical deciduous forest.

Elevation: $518 \mathrm{~m}$.

Collection dates: 1964: VII.24, VIII.24; 1987: VII.30VII.31; 1989.VII.08.

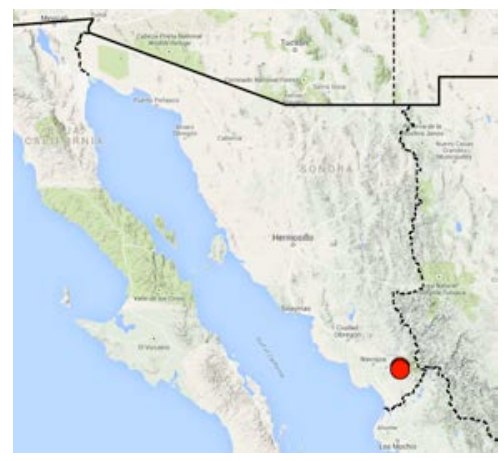

230. Selenophorus (Hemisopalus) sp. 2

Number examined: 1.

Municipality: Álamos.

Habitat: [at light]

Vegetation: tropical deciduous forest.

Elevation: $518 \mathrm{~m}$.

Collection date: 1987: VII.30-VII.31.

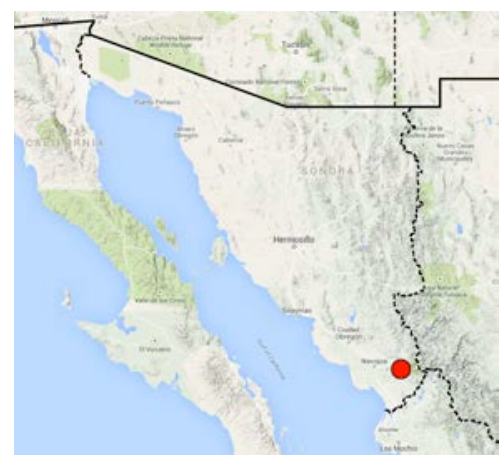


231. Selenophorus (Hemisopalus) sp. 3

Number examined: 38 .

Municipality: Álamos.

Habitat: [at light]

Vegetation: tropical deciduous forest.

Elevation:?

Collection date: 1964: VIII.24.

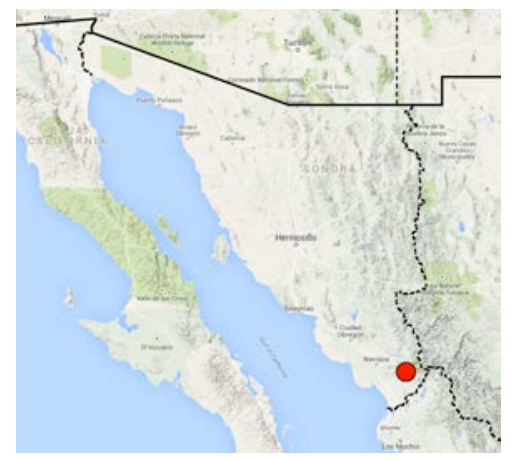

232. Selenophorus (s. str.) sp. 4

Number examined: 1 .

Municipality: Navojoa.

Habitat: [at light]

Vegetation: coastal thornscrub.

Elevation range: ?

Collection date: 1964: VIII.25.

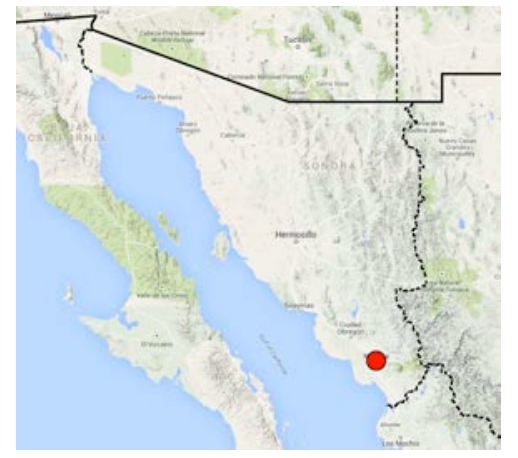

233. Selenophorus (s. str.) sp. 25

Number examined: 1.

Municipality: Agua Prieta.

\section{Habitat: ?}

Vegetation: desert grassland.

Elevation: $1600 \mathrm{~m}$.

Collection date: 1982.VIII.22.

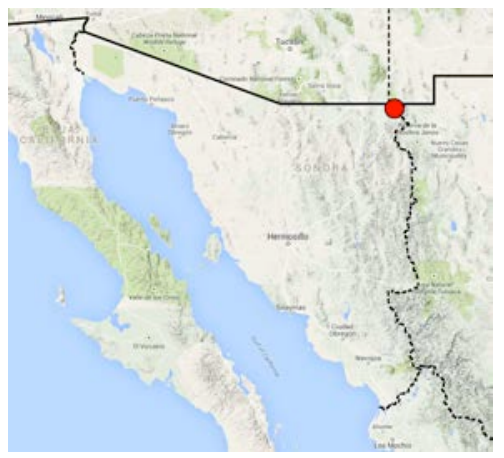

234. Selenophorus (s. str.) sp. 26

Number examined: 4.

Municipalities: Ímuris, Moctezuma, Nácori Chico.

Habitat: ?

Vegetation: cottonwood-willow riparian deciduous forest; foothills thornscrub; oak woodland.

Elevation range: $500-1380 \mathrm{~m}$.

Collection dates: 1982: VIII.02, VIII.23; 1983.VII.04; 1993.VII.05.

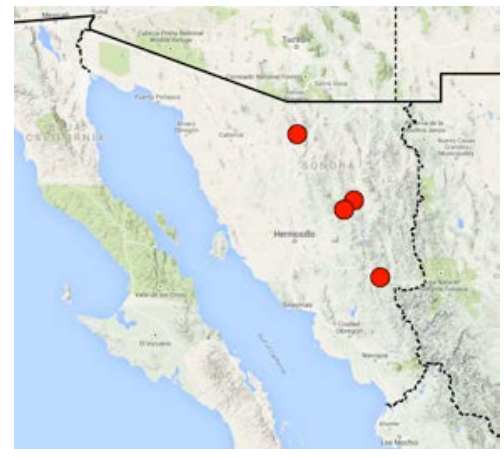

235. Stenolophus comma (Fabricius, 1775)

Number examined: 9.

Municipalities: Agua Prieta, Nácori Chico, Santa Ana.

Habitat: [at light]

Vegetation: Chihuahuan and Sonoran desertscrub; pineoak forest.

Elevation range: $1350-1950 \mathrm{~m}$.

Collection dates: 1982: VIII.06-VIII.07, VIII.23; 1983. VII.04.

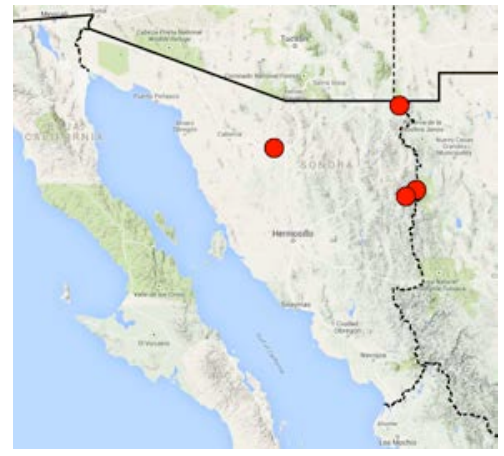


236. Stenolophus lineola (Fabricius, 1775)

Number examined: 2.

Municipalities: Nácori Chico.

Habitat: rocky stream canyon. [at light]

Vegetation: cypress-maple-alder-sycamore riparian forest; pine-oak forest.

Elevation Range: 1450 - 1648 m.

Collection dates: 1983.VII.041 2018.VIII.05.

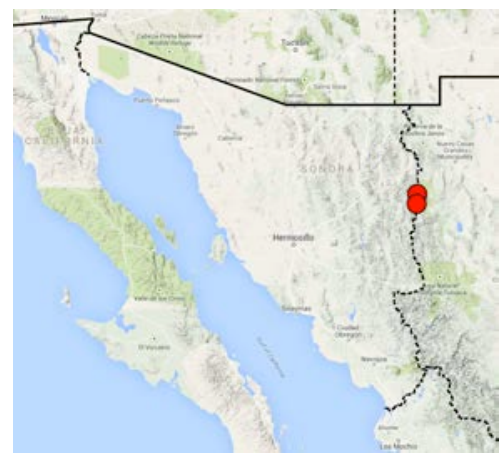

237. Stenolophus ochropezus (Say, 1823)

Number examined: 39.

Municipalities: Agua Prieta, Cananea, Ímuris.

Habitat: rocky hillside. [at light]

Vegetation: Chihuahuan desertscrub; cottonwood-willow riparian forest; desert grassland; oak woodland.

Elevation range: $1040-1350 \mathrm{~m}$.

Collection dates: 1982: VIII.19-VIII.20, VIII.24; 1986. VIII.30; 2014.VIII.14; $2017 . I V .23$.

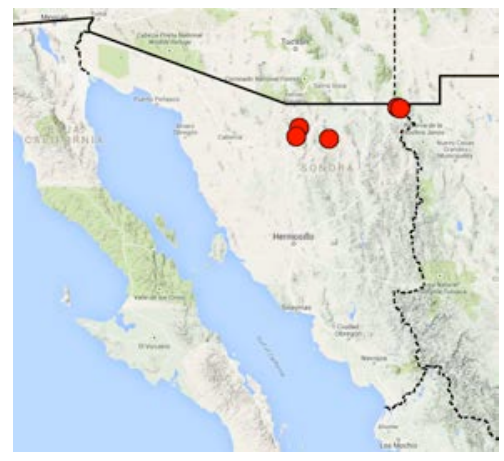

238. Stenolophus conjunctus species group sp.

Number examined: 2.

Municipalities: Agua Prieta, Moctezuma.

Habitat: ?

Vegetation: desert grassland; foothills thornscrub.

Elevation range: $1000-1600 \mathrm{~m}$.

Collection dates: 1982.VIII.22; 1993.VII.05.

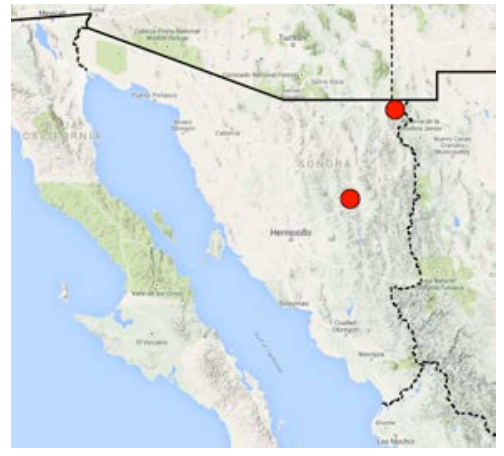

239. Stenomorphus californicus rufipes LeConte, 1858

Number examined: 1.

Municipality: Navojoa.

Habitat: [at light]

Vegetation: coastal thornscrub.

Elevation: ?

Collection date: 1962.VII.12.

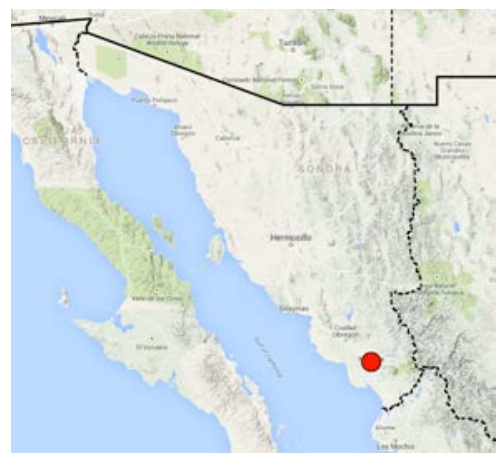

240. Stenomorphus convexior Notman, 1922

Number examined: 72.

Municipalities: Agua Prieta, Álamos, Altar, Cananea, Fronteras, Magdaleno de Kino, Mazatán, Naco, Nogales.

Habitat: lights at building at night; rocky stream canyon and hillside. [at light]

Vegetation: Chihuahuan and Sonoran desertscrub; desert grassland; foothills thornscrub; oak woodland; tropical deciduous forest.

Elevation range: $376-1521 \mathrm{~m}$.

Collection dates: 1965.VII.31; 1967.VIII.20; 1971. VIII.16; 2012: VIII.01, VIII.09, VIII.13, IX.05, IX.12; 2013: VIII.23, IX.11; 2014: IX.14, IX.16; 2015: VIII.08, VIII.13, IX.06; 2016.VIII.24; 2017.IX.02; 2018.VIII.13. 


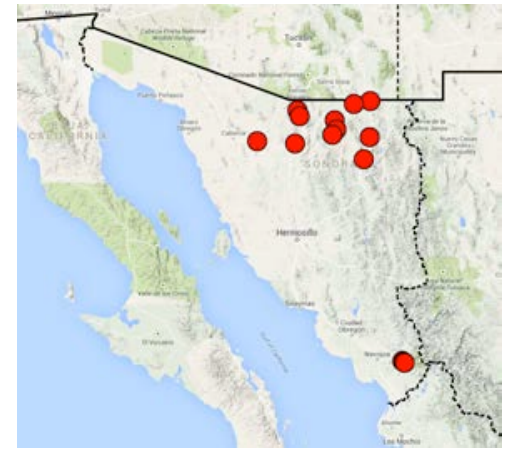

241. Stenomorphus sinaloae Darlington, 1936 Number examined: 19.

Municipalities: Mazatán, Quiriego, Rayón.

Habitat: urban.

Vegetation: coastal and foothills thornscrub.

Elevation range: $542-550 \mathrm{~m}$.

Collection dates: 1991.VIII.31; 2012.VIII.13; 2014. VIII.29.

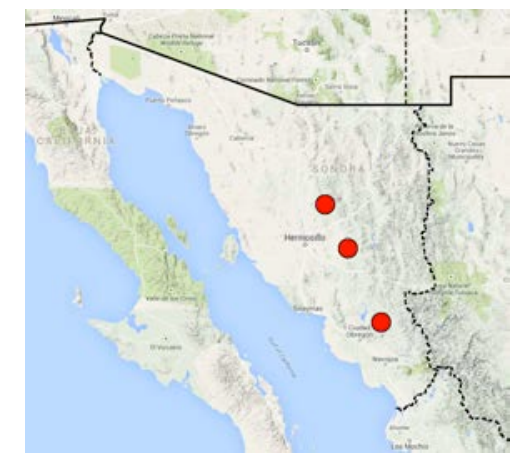

242. Trichopselaphus erwinorum Ball, 1978

Number examined: 1.

Municipality: Álamos.

Habitat: [at light]

Vegetation: tropical deciduous forest.

Elevation: $1577 \mathrm{~m}$.

Collection date: 1989: VII.11-VII.13.

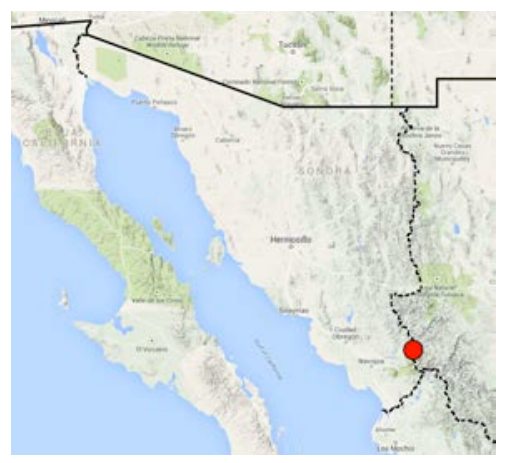

\section{Sphodrini}

243. Calathus mcclevei, new species

http://zoobank.org:/B205C63B-EBBA-4F2D-BBBC200FD6AAA430

Fig. 11A, 12A, 12B

Etymology: Latinized surname name of Scott McCleve, one of the collectors of the type series.

Type material: 37 specimens. Holotype male, labelled: "MEXICO: Sonora, S.M./ Occidental, $10 \mathrm{~km} \mathrm{~s} /$ Yecora, 1830 m/oak-pine litter/ 93-17, 06-07.VII.93"; "MEX. EXPEDITION/ 1993, S. McCleve,/ G.E. \& K.E. Ball/ collectors"; "UASM\#/ 259363" (UNAM). Paratypes, 36: 9 males, labelled same as holotype, except: "UASM\#/ 259385" (CASC); "UASM\#/ 259381" (UAIC); "UASM\#/ 259361", "UASM\#/ 259368", "UASM\#/ 259372", "UASM\#/ 259374", "UASM\#/ 259380", “UASM\#/ 259382" (UASM); “UASM\#/ 259384" (USNM). 25 females, labelled same as holotype, except: "UASM\#/ 259360" (CASC); "UASM\#/ 259362" (UAIC); "UASM\#/ 259364" (UNAM); "UASM\#/ 259366", "UASM\#/ 259367", "UASM\#/ 259369", “UASM\#/ 259370", "UASM\#/ 259371", "UASM\#/ 259376" - "UASM\#/ 259379", "UASM\#/ 259383", "UASM\#/ 259386" - "UASM\#/ 259394" (UASM); "UASM\#/ 259365" (USNM). 1 male: "MEXICO SONORA/ $4.5 \mathrm{~km}$ n Mesa de/ Tres Rios, oak-/ pine for.; 1950 m./July 6, 1983, 83-12"; "MEXICO EXPED. 1983/ H.E. Frania,/ R.J. Jaaguagi,/ D. Shpeley colls."; "UASM\#253979" (UASM). 1 female: "MEXICO SONORA/ $4.5 \mathrm{~km}$ n Mesa de/ Tres Rios, oak-/ pine for.; 1950 m./ July 6, 1983, 83-12"; "MEXICO EXPED. 1983/ H.E. Frania,/ R.J. Jaagumagi,/ D. Shpeley colls.”; "UASM\#/ 253980" (UASM).

Type locality: Mexico, Sonora, Municipio de Yécora, 10 $\mathrm{km}$ south of Yécora, ca. $28.278^{\circ} \mathrm{N} 108.926^{\circ} \mathrm{W}$.

Diagnosis: Males of this species are readily separated from males of C. tigrinus, new species, by morphology of the male genitalia: apical portion short, LAP/WD 1.782.43; median lobe shorter (LML) relative to body length (TL) of male (ratio LML/TL 0.22-0.26).

Description: Member of the melanocephalus group (Ball \& Negre 1972:428-429). Total Length: males $(n=10)$, 7.76-9.20 mm (mean $8.51 \mathrm{~mm})$; females $(\mathrm{n}=10), 8.28$ $9.64 \mathrm{~mm}$ (mean $9.06 \mathrm{~mm}$ ). Mouthparts, antennae and legs rufo-testaceus to nearly brunneus. Dorsal (Figure 10A) and ventral surface rufo-brunneus to dark brunneus, lateral margins of pronotum and elytra diffusely paler. Dorsal microsculpture with isodiametric sculpticels; males with head and pronotum shiny, elytra dull; females with head and pronotum less shiny than in males, elytra dull. Pronotum basally with coarser sculpticels in both sexes. Prosternum with apex beaded. Elytra with 3 to 5 setae in striae 3 (preapical seta also observed in stria 2 and interval 3 ). Hind wings reduced to narrow stubs, adults incapable of flight. Middle femur with 2 setae in antero-ventral row. Hind metatarsus with groove on 
both outer and inner surfaces. Tarsal claws finely pectinate. Male genitalia (Figure 12A, 12B) with short apical portion and well developed apical disc; endophallus without spines; left paramere somewhat ovoid, with membranous lobe apically; right paramere narrow, elongate, with short spine at apex. Female ovipositor was not studied.

Municipalities: Nácori Chico, Yécora.

Habitat: ?

Vegetation: oak-pine forest; oak-pine litter.

Elevation range: $1830-1950 \mathrm{~m}$.

Collection dates: 1983.VII.06; 1993: VII.06-VII.07.

Distribution: This species is currently only known from Sonora and near Madera in Chihuahua.

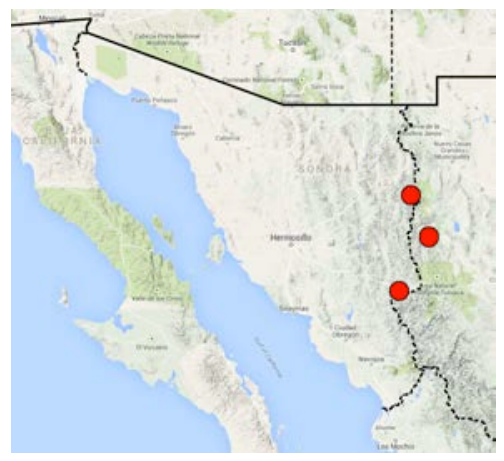

244. Calathus opaculus LeConte, 1854

Number examined: 30 .

Municipalities: Agua Prieta, Ímuris.

Habitat: flood litter

Vegetation: Chihuahuan desertscrub; cottonwood-willow riparian.

Elevation range: $963-1350 \mathrm{~m}$.

Collection dates: 1982: VIII.20, VIII.24; 1986.VIII.30.

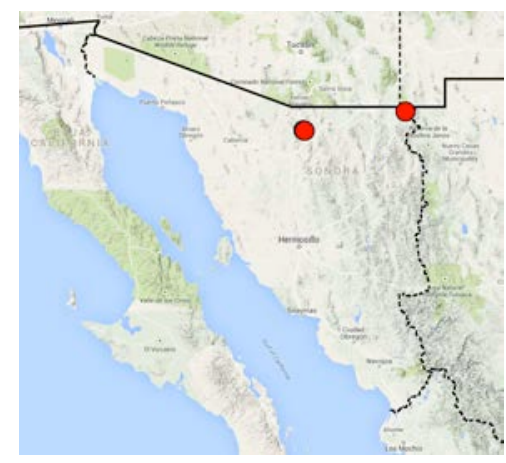

245. Calathus ruficollis Dejean, 1828

Number examined: 1.

Municipality: Cananea.

Habitat: rocky stream canyon.

Vegetation: oak woodland; pine-oak forest; riparian deciduous forest;

Elevation: $1905 \mathrm{~m}$.

Collection date: 2014.VIII.24.

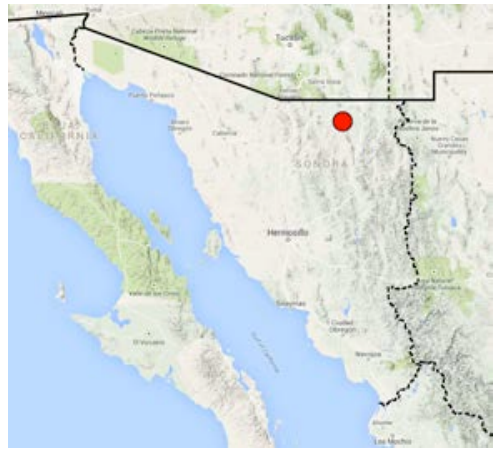

246. Calathus tigrinus, new species http://zoobank.org/0C39C5D9-FE17-4429-A7D6A21E2E0E5E76

Fig. 11B, 12C, 12D

Etymology: Latinized name of the mountain range in which this species was collected, the Sierra El Tigre in Sonora.

Type material: 21 specimens. Holotype male, labelled: "MEXICO: Sonora,/ Sierra El Tigre,/ $30.58988^{\circ} \mathrm{N}$ $109.20811^{\circ} \mathrm{W}$, camp, large flat area,"; "pine-oak forest, at night/ headlamping, on ground/2261m,12.VIII.2015,/ D. Shpeley11-15'; "UASM\#370301" (UNAM). Paratypes, 20: 3 males, labelled same as holotype, except: "UASM\#/ 370303 -UASM\#/ 370305" (UASM). 2 females, labelled same as holotype, except: "UASM\#/ 370300" (UASM); “UASM\#/ 370302" (UNAM). 2 males: "MEX. Sonora Sierra/ Huachinera 56 km./ n.e. Nacori Chico/ oak-pine forest/ 2330 m. 82-12/ August 8, 1982"; "MEX. EXPED. 1982/ G.E. \& K.E. Ball/ \& S. McCleve/ collectors": "UASM\#/ 253816" (CASC); "UASM\#/ 253815" (UASM). 3 females: "MEX. Sonora Sierra/ Huachinera 56km./ n.e. Nacori Chico/ oak-pine forest/ 2330 m. 82-12/ August 8, 1982"; "MEX. EXPED. 1982/ G.E. \& K.E. Ball/ \& S. McCleve/ collectors": "UASM\#/ 253814" (CASC); "UASM\#/ 253812, UASM\#/ 253813" (UASM). 1 female: "MEX. Sonora Sierra/ Huachinera 32-34/ km. n.e. Nacori/ Chico 1950 m.; oak-/ pine for. 82-10/ August 6\&7, 1982"; "MEX. EXPED. 1982/ G.E. \& K.E. Ball/ \& S. McCleve/ collectors"; "UASM\#/ 253780" (UASM). 3 males: "MEXICO Sonora/ $18.5 \mathrm{~km}$ e Mesa de/ Tres Rios, pine-oak/ forest; 2440 m./ July 7, 1983 83-18"; "MEXICO EXPED. 1983/ H.E. Frania,/ R.J. Jaagumagi,/ D. Shpeley colls.": "UASM\#/ 253984" (UAIC); "UASM\#/ 253988" (UASM); “UASM\#/ 253987” (USNM). 5 females: "MEXICO Sonora/ $18.5 \mathrm{~km}$ e Mesa de/ Tres Rios, pine-oak/ forest; 2440 m./ July 7, 1983 83-18"; "MEXICO EXPED. 1983/ H.E. Frania,/ R.J. Jaagumagi,/ D. Shpeley colls.": "UASM\#/ 253986" (UAIC); "UASM\#/ 253981" - "UASM\#/ 253983" (UASM); "UASM\#/ 253985" (USNM). 1 male: MEX. Sonora Sierra/ San Luis; Varela/ Rch., Cn. Chimeneaz/ 14601690m. oak-/ juniper-sycamore/ VIII.19.1982 82-20"; "MEX. EXPED 1982/ GE Ball, S. McCleve/ \& DR Maddison/ collectors"; “UASM\#253779” (UASM). 
Type locality: Mexico, Sonora, Municipio de Nacozri de García, Rancho El Tigre, 28.3 km (by air) WNW Bavispe, Sierra El Tigre, $30.58988^{\circ} \mathrm{N} 109.20811^{\circ} \mathrm{W}$.

Diagnosis: Males of this species are readily separated from males of $C$. mcclevei, new species, by morphology of the male genitalia: apical portion long, LAP/WD 2.563.63; median lobe longer (LML) relative to body length (TL) of male (ratio LML/TL 0.29-0.32).

Description: Member of the melanocephalus group (Ball \& Negre 1972:428-429). Total Length: males $(\mathrm{n}=10)$, 7.92-9.96 mm (mean $8.95 \mathrm{~mm})$; females $(\mathrm{n}=11)$, 7.48$9.76 \mathrm{~mm}$ (mean $8.56 \mathrm{~mm}$ ). Mouthparts, antennae and legs rufo-testaceus to nearly brunneus. Dorsal (Figure 10B) and ventral surface rufo-brunneus to dark brunneus, lateral margins of pronotum and elytra diffusely paler. Dorsal microsculpture with isodiametric sculpticels; males with head, pronotum and elytra shiny; females with head and pronotum shiny, elytra dull. Pronotum basally with coarser sculpticels in both sexes. Prosternum with apex beaded. Elytra with 3 to 5 setae in stria 3 (preapical seta also observed in stria 2 and interval 3 ). Hind wings reduced to narrow stubs, adults incapable of flight. Middle femur with 2 setae in antero-ventral row. Hind metatarsus with groove on both outer and inner surfaces. Tarsal claws finely pectinate. Male genitalia (Figure 12C, 12D) with long apical portion and well developed apical disc; endophallus without spines; left paramere somewhat ovoid, with membranous lobe apically; right paramere narrow, elongate, with short spine at apex. Female ovipositor was not studied.

Municipalities: Agua Prieta, Nácori Chico, Nacozari de García.

Habitat: large flat area near ridge top; shallow rocky arroyo.

Vegetation: oak-pine forest; oak-juniper-pine forest; oak-juniper-sycamore forest; pine-oak forest.

Elevation range: $1460-2440 \mathrm{~m}$.

Note regarding elevation: Ball and Negre (1972:522) stated that the Mexican species of Calathus are not found below about 6,500 feet (based on the specimens they used for their study). The single male specimen collected at the Varela Ranch $(4,888-5,544$ feet) is the only specimen of this new species that was collected below that elevation.

Collection dates: 1982: VIII.06-VIII.08, VIII.19; 1983. VII.07; 2015: VIII.11-VIII.12.

Distribution: This species is currently only known from Sonora and near Madera in Chihuahua.

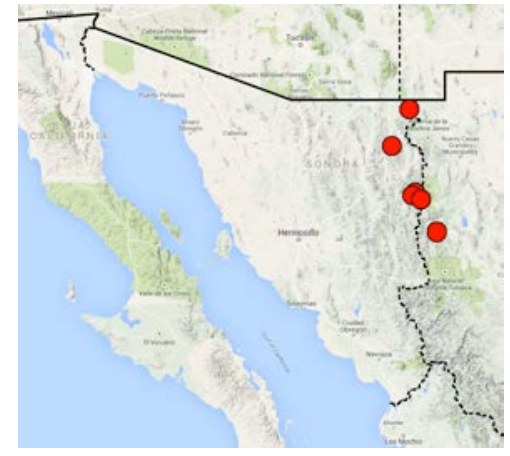

Ball \& Negre (1972:421-423) used morphology of the median lobe (male genitalia) and distribution records to separate some of the species in their key, as differences in external morphological characters were lacking. The following changes have to be made to the key to the Nearctic species of Calathus in Ball and Nègre (1972:422) to accommodate the above two new species:

14 (13) Specimen from Sierra Madre Occidental (Chihuahua, Durango or Sonora) .... 15

14'Specimen from Trans-Volcanic Sierra or Sierra Madre Oriental 16

15 (14) Pronotum with disc more convex, lateral depression not widened basally, posteriolateral impression convex (Chihuahua) ……………. C. ambigens (in part) Bates

15' Pronotum with disc less convex, lateral depression widened basally, posteriolateral impression concave to slightly convex, not as above (Chihuahua, Durango or Sonora) $15 \mathrm{a}$ 15a (15) Specimen from Durango

C. durango Ball \& Nègre

15a' Specimen from Sonora or Chihuahua ................... 15b

$15 \mathrm{~b}$ (15a') Median lobe with apical portion short as in Figure 11A, 11B (ratio LAP/WD 1.78-2.43), median lobe shorter (LML) relative to body length (TL) of male (ratio LML/TL 0.22-0.26) ......... C. mcclevei, new species

15b' Median lobe with apical portion long as in Figure 11C, 11D (ratio LAP/WD 2.56-3.63), median lobe longer (LML) relative to body length (TL) of male (ratio LML/ TL 0.29-0.32) C. tigrinus, new species

\section{Calathus sp. UASM son-3}

Number examined: 1

Municipality: Cananea.

Habitat: ?

Vegetation: pine-oak forest.

Elevation: $2350 \mathrm{~m}$.

Collection dates: 1982.VIII.25

Note: The specimen is a female that cannot be assigned at this time to any of the known or undescribed taxa. 

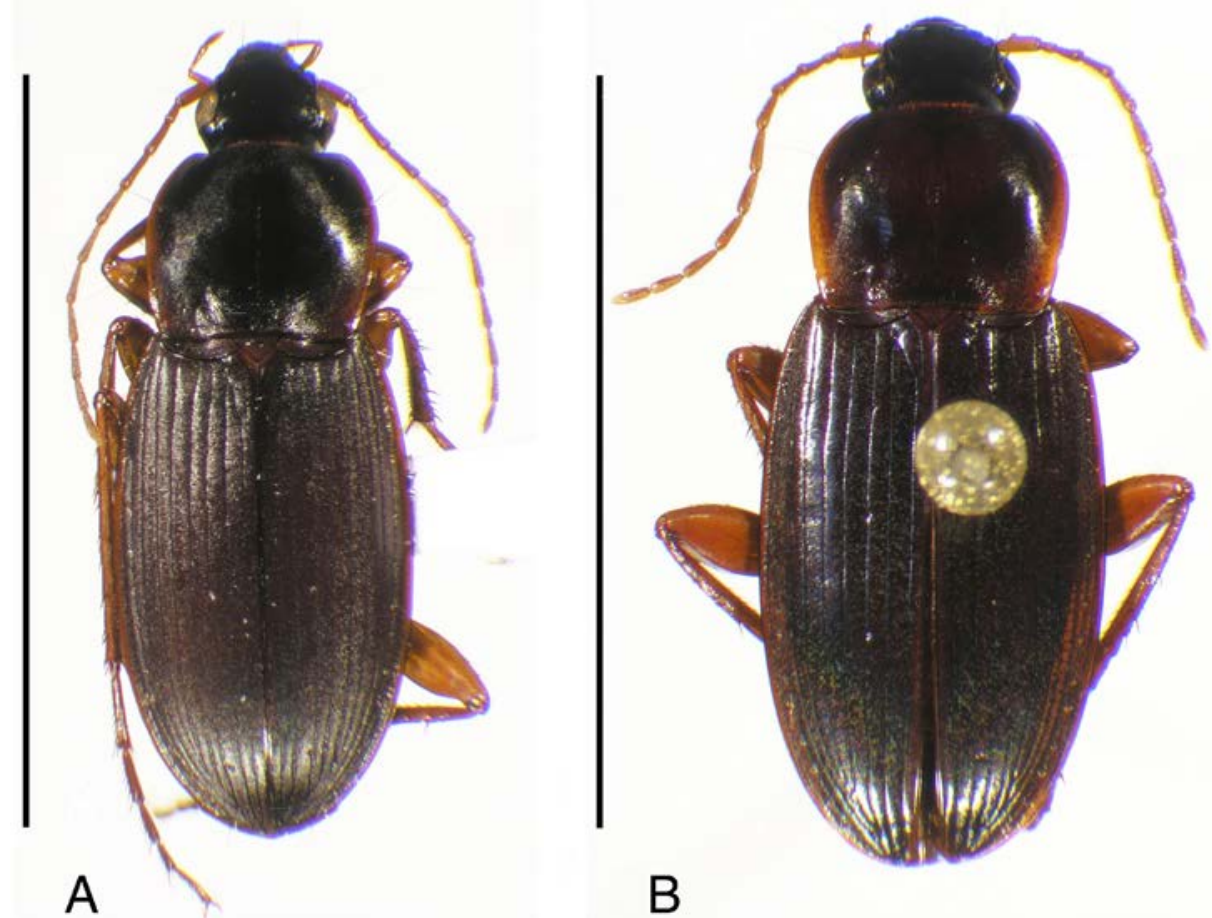

Figure 11. Habitus digital image of Calathus species, dorsal aspect. A, Holotype of C. mcclevei, new species; B, Holotype of C. trigrinus, new species. Scale bar $9 \mathrm{~mm}$.
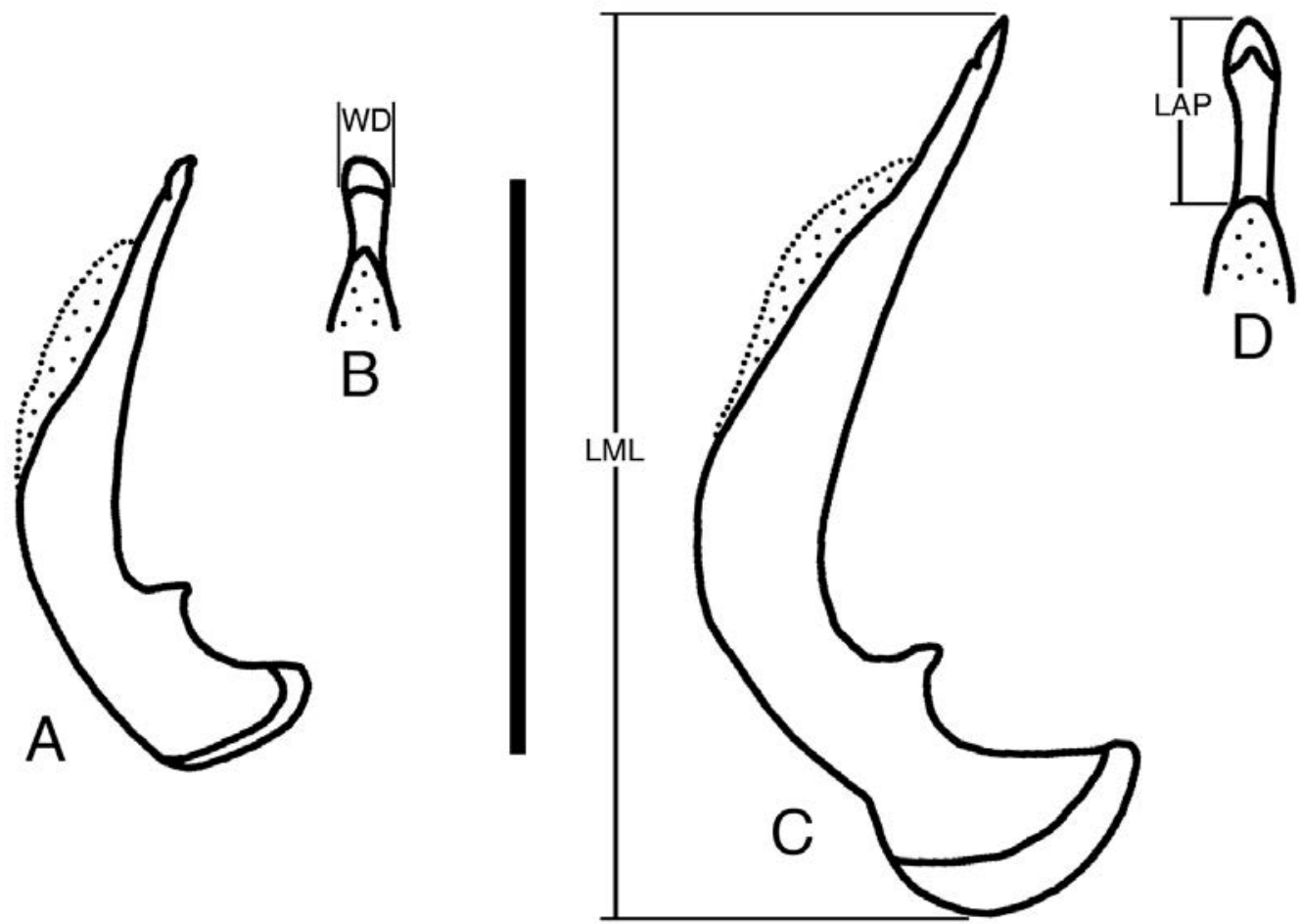

Figure 12. Line drawings of male genitalia of Calathus species. A, C left lateral aspect, and $\mathbf{B}, \mathbf{D}$ dorsal aspect. A, B C. mcclevei, new species. C, D C. tigrinus, new species. Legend: LAP length of apical portion; LD length of disc; LML length of median lobe; WD width of disc. Scale bar $2 \mathrm{~mm}$. 


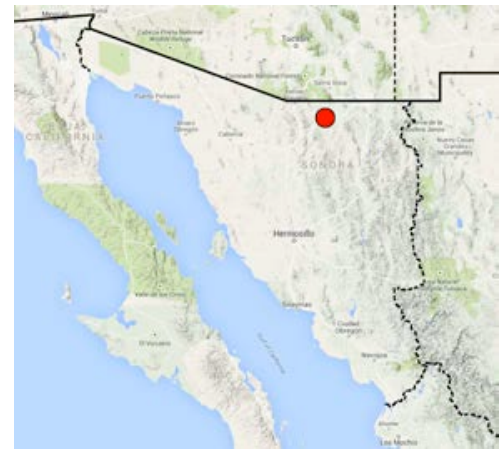

248. Synuchus semirufus (Casey, 1913)

Number examined: 16.

Municipalities: Cananea, Huachinera, Nácori Chico, Nacozari de García.

Habitat: meadow; rocky arroyo, slope, and ridge top.

Vegetation: pine-oak forest.

Elevation range: $2090-2440 \mathrm{~m}$.

Collection dates: 1982: VIII.04, VIII.08; 1983.VII.07;

2013: IX.09-IX.10; 2015: VIII.10-VIII.11.

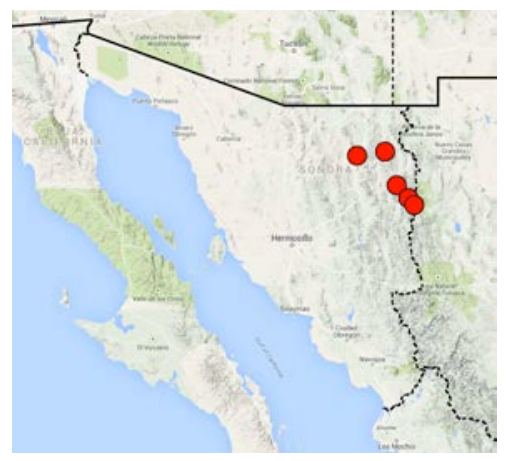

26.Platynini

249. Agonum anthracinum Dejean, 1831

Number examined: 16.

Municipalities: Aconchi, Cananea, Huachinera, Nácori Chico, Nacozari de García, Yécora.

Habitat: rocky arroyo, mountainside ridge.

Vegetation: desert grassland; foothills thornscrub; oak woodland; pine-oak forest.

Elevation range: $1150-2467 \mathrm{~m}$.

Collection dates: 1982: VIII.05, VIII.11; 1983.VII.06;

1990: VI.30-VII.03; 2012.IX.03; 2013.VII.17; 2015:

VIII.12, IX.06; 2017: VII.15; VIII.12-VIII.13.

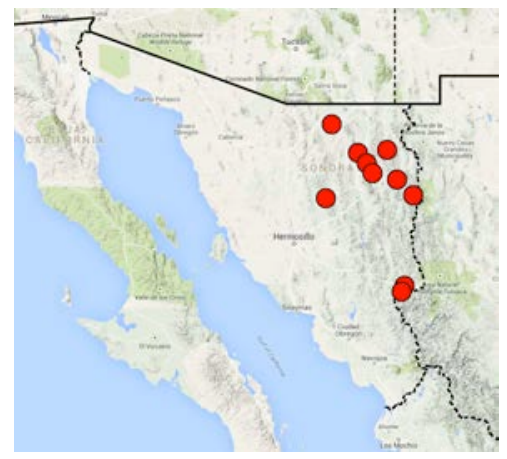

250. Agonum cyanope (Bates, 1882)

Number examined: 13.

Municipalities: Ímuris, Nácori Chico, Nacozari de García, Yécora.

Habitat: camp clearing; clearing; gentle rocky slope.

Vegetation: Cypress-maple-alder-sycamore riparian forest; oak woodland; pine-oak forest.

Elevation range: $1400-1648 \mathrm{~m}$.

Collection dates: 1956.XII.26; 1982.VIII.11; 2014.IX.20; 2018: VIII.06, VIII.09.

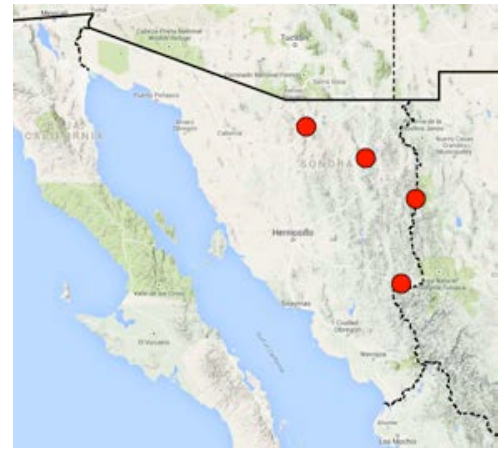

251. Agonum cyclifer (Bates, 1884)

Number examined: 1.

Municipality: Benjamín Hill.

Habitat: plains.

Vegetation: Sonoran desertscrub.

Elevation: $741 \mathrm{~m}$.

Collection date: 2017.VII.23.

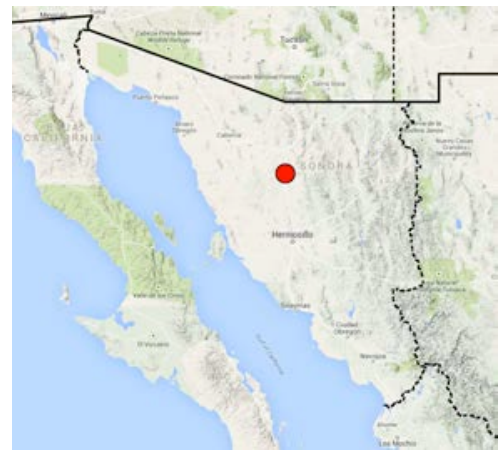


252. Agonum decorum (Say, 1823)

Number examined: 13.

Municipalities: Agua Prieta, Ímuris.

Habitat: [at light]

Vegetation: cottonwood-willow riparian forest; oak woodland.

Elevation: $1291 \mathrm{~m}$.

Collection dates: 1956.XII.26; 2017.IV.23.

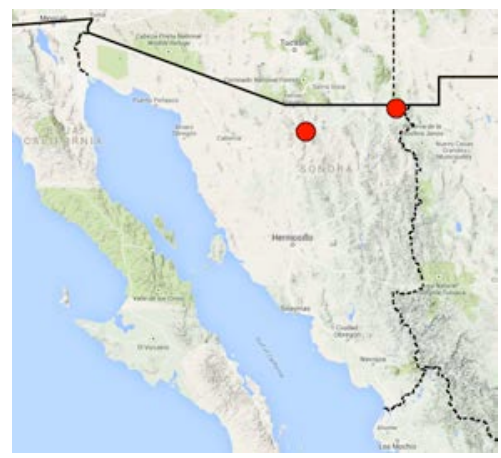

253. Agonum extensicolle (Say, 1823)

Number examined: 18.

Municipalities: Cananea, Ímuris, Nácori Chico, Santa Ana.

Habitat: gentle rocky slopes; stream margin.

Vegetation: desert grassland; foothills thornscrub; Sonoran desertscrub.

Elevation range: $1450-1525 \mathrm{~m}$.

Collection dates: 1956.XII.26; 1983.VII.05; 1985.VIII.01; 2014.VIII.27.

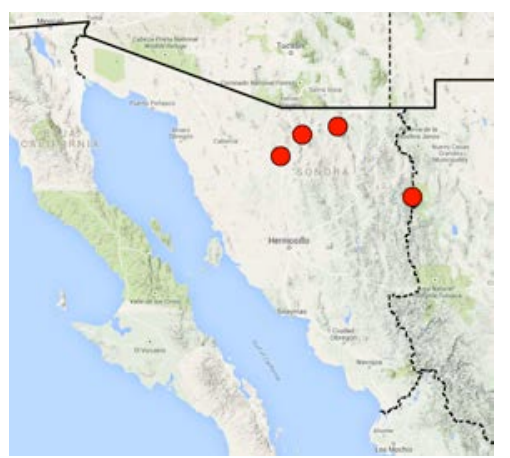

254. Agonum parextimum Liebherr, 1986

Number examined: 8 .

Municipalities: Carbó, Hermosillo, Santa Ana.

Habitat: urban; plains. [at light]

Vegetation: Sonoran desertscrub.

Elevation range: $255-710 \mathrm{~m}$.

Collection dates: 1953: VII.09-VII.16; 1958.IV.03; 1960.

VIII.08; 1982.VIII.23; 2017.VII.21.

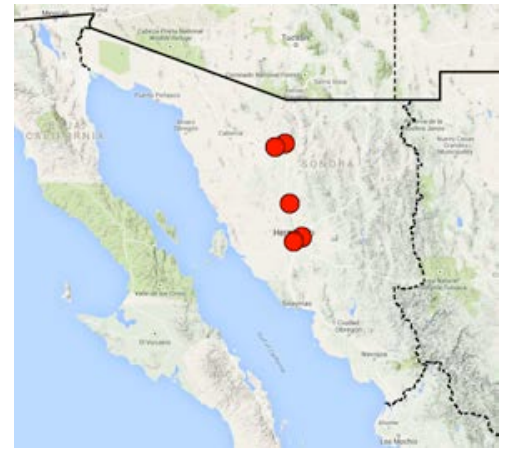

255. Agonum placidum (Say, 1823)

Number examined: 4.

Municipality: Huachinera.

Habitat: meadow.

Vegetation: pine-oak forest.

Elevation: $2090 \mathrm{~m}$.

Collection date: 1982.VIII.04.

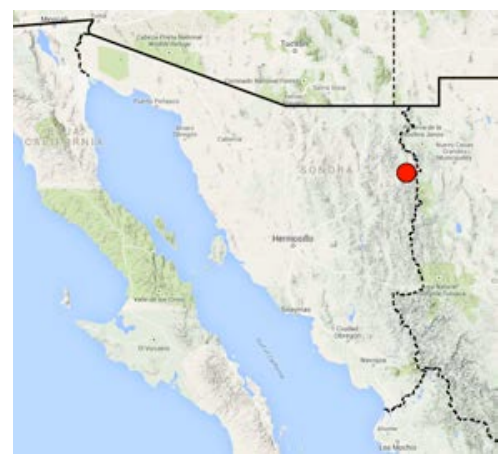

256. Agonum punctiforme (Say, 1823)

Number examined: 19.

Municipality: Ímuris.

Habitat: ?

Vegetation: cottonwood-willow riparian forest.

Elevation: $1040 \mathrm{~m}$.

Collection date: 1982.VIII.24.

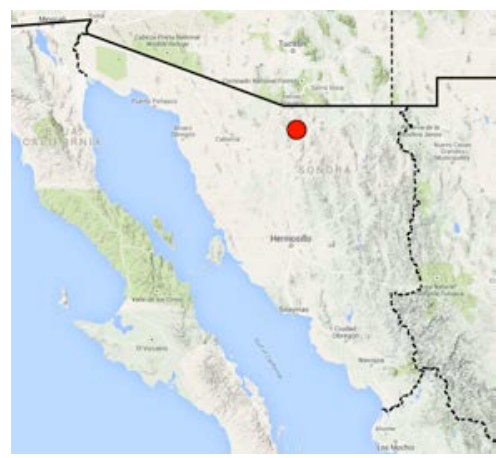

257. Agonum texanum LeConte, 1878

Number examined: 4.

Municipalities: Agua Prieta, Moctezuma, Santa Ana.

Habitat: [at light]

Vegetation: Chihuahuan and Sonoran desertscrub; foothills 
thornscrub.

Elevation: 944 - $1350 \mathrm{~m}$.

Collection dates: 1958.IV.03; 1980: VII.21-VII.22; 1982:

VIII.19-VIII.20.

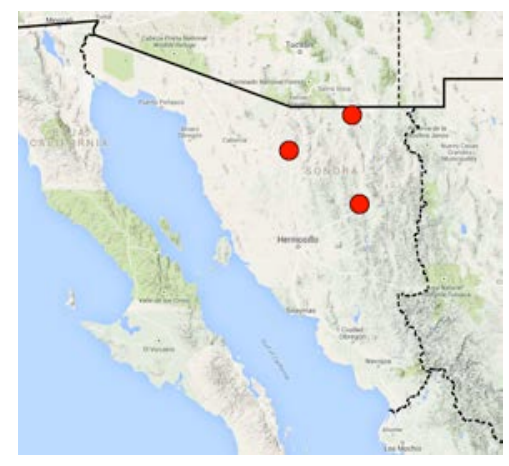

258. Onypterygia cyanea Chaudoir, 1878

Number examined: 3 .

Municipality: Álamos.

Habitat: ?

Vegetation: tropical deciduous forest.

Elevation:?

Collection date: 1935.VII.20.

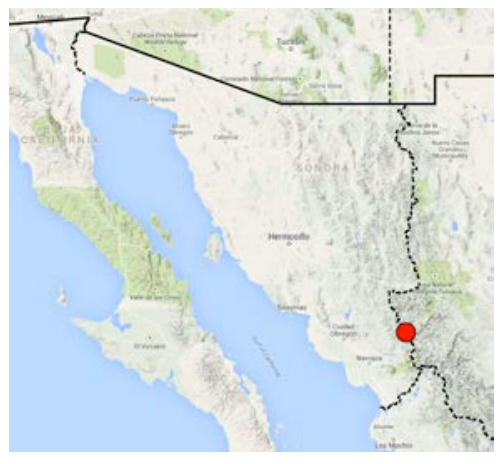

259. Onypterygia famini Solier, 1835

Number examined: 7.

Municipalities: Álamos, Yécora.

Habitat: [at light]

Vegetation: oak woodland; pine-oak forest; tropical deciduous forest.

Elevation range: $1547-1752 \mathrm{~m}$.

Collection dates: 1989: VII.11-VII.13; 1990: VI.30VII.03; 1993: VII.08-VII.09.

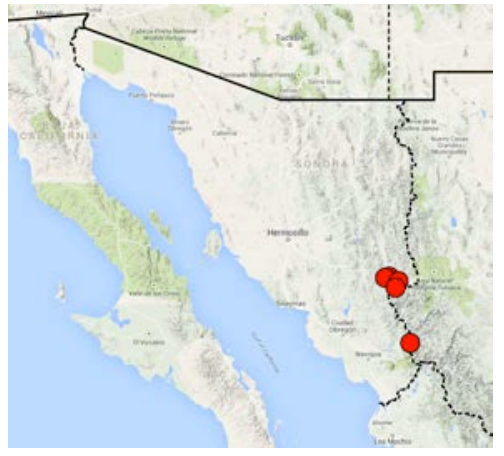

260. Onypterygia fulgens Dejean, 1831

Number examined: 2.

Municipality: Álamos.

Habitat: ?

Vegetation: tropical deciduous forest.

Elevation:?

Collection date: 1935.VII.20.

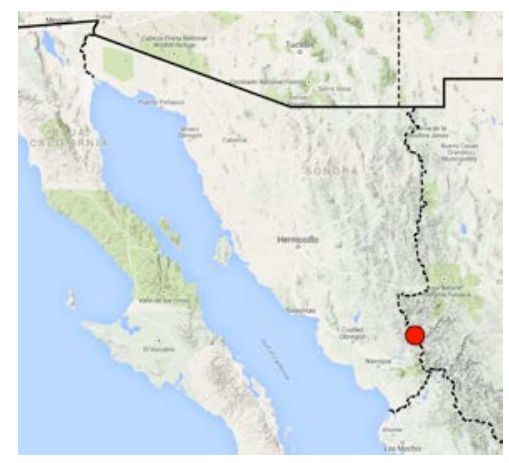

261. Onypterygia tricolor Dejean, 1831

Number examined: 2

Municipality: Álamos.

Habitat: [at light]

Vegetation: tropical deciduous forest.

Elevation range: $435-1577 \mathrm{~m}$.

Collection dates: 1989: VII.11-VII.13; 2019.VII.25.

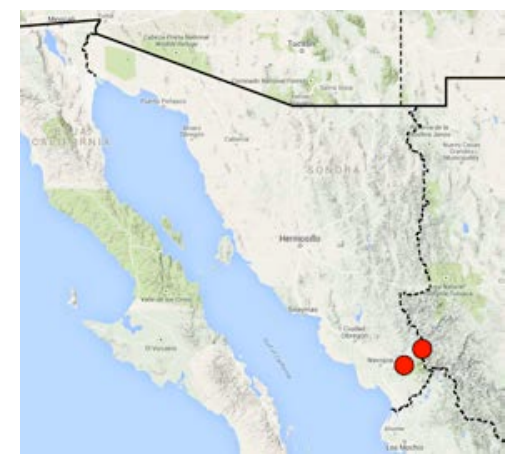

262. Hemiplatynus chihuahuae (Bates, 1884)

Number examined: 5 .

Municipalities: Huachinera, Nácori Chico, Yécora.

Habitat: arroyo. [at light]

Vegetation: pine-oak forest. 
Elevation range: $1646-2090 \mathrm{~m}$.

Collection dates: 1982: VIII.03-VIII.04; 1983.VII.06; 1990: VI.28-VI.29.

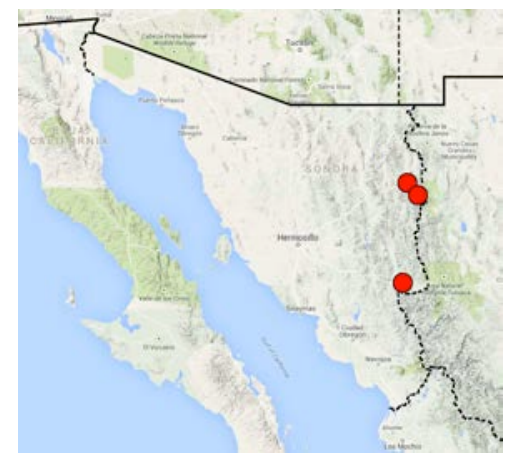

263. Platynus acuminatus (Chevrolat, 1835)

Number examined: 13.

Municipalities: Álamos, Fronteras, Nácori Chico, Yécora.

Habitat: cow pasture; rocky arroyo and stream canyon. [at light]

Vegetation: cypress-maple-alder-sycamore riparian forest; pine-oak forest; tropical deciduous forest.

Elevation range: $1429-1752 \mathrm{~m}$.

Collection dates: 1989: VII.11-VII.13; 1990: VII.02VII.03; 2016: VII.02, VIII.25; 2018: VIII.05, VIII.09.

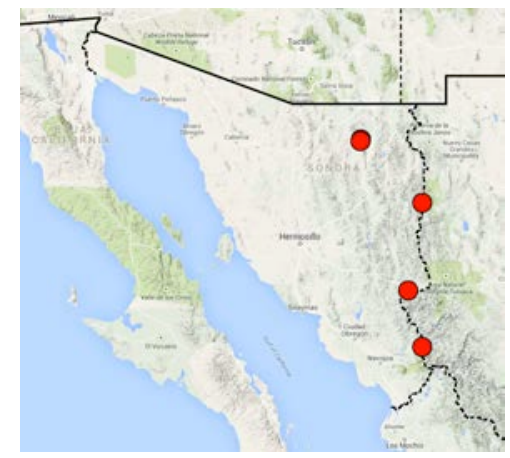

264. Platynus brunneomarginatus (Mannerheim, 1843)

Number examined: 1.

Municipality: Ímuris

Habitat: ?

Vegetation: cottonwood-willow riparian forest.

Elevation: $1040 \mathrm{~m}$.

Collection date: 1982.VIII.24.

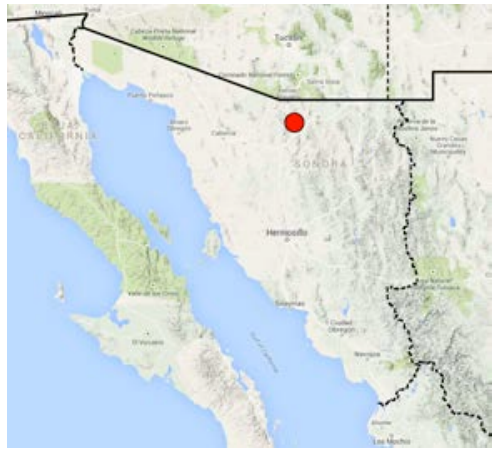

265. Platynus falli (Darlington, 1936)

Number examined: 6 .

Municipalities: Arizpe, Moctezuma, Nacozari de García, Yécora.

Habitat: rocky slope. [at light]

Vegetation: desert grassland; foothills thornscrub.oak woodland;

Elevation range: $500-1413 \mathrm{~m}$.

Collection dates: 1982.VIII.02; 1984.VIII.24; 2015. VIII.08; 2019.VII.06.

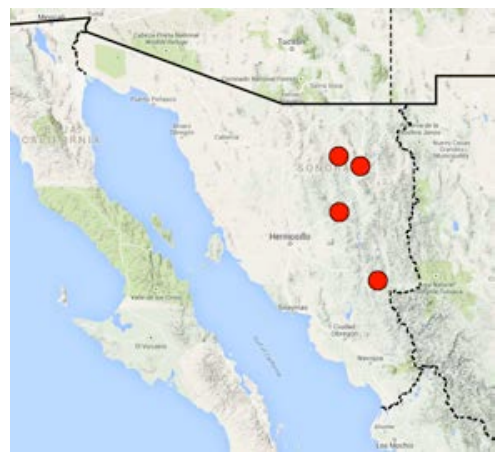

266. Platynus lyratus (Chaudoir, 1878)

Number examined: 117.

Municipalities: Agua Prieta, Álamos, Fronteras, Ímuris, Moctezuma, Nácori Chico, Nacozari de García, Yécora.

Habitat: bog; rocky arroyo, mountainside and stream canyon; stream margin. [at light]

Vegetation: Chihuahuan desertscrub; cottonwood deciduous riparian forest; cypress-maple-alder-sycamore riparian forest; oak woodland; pine-oak forest; tropical deciduous forest.

Elevation range: $944-1950 \mathrm{~m}$.

Collection dates: 1982: VIII.06-VIII.07, VIII.20, VIII.24; 1983:VII.04, VII.11-VII.12; 1986.VIII.30; 1989: VII.11-VII.13; 1990: VI.30-VII.03; 1993: VII.06VII.07; 2013.IX.08; 2015.VIII.12; 2016: VIII.14, VIII.16; 2017.VIII.15; 2018: VIII.05-VIII.06, VIII.08VIII.09. 


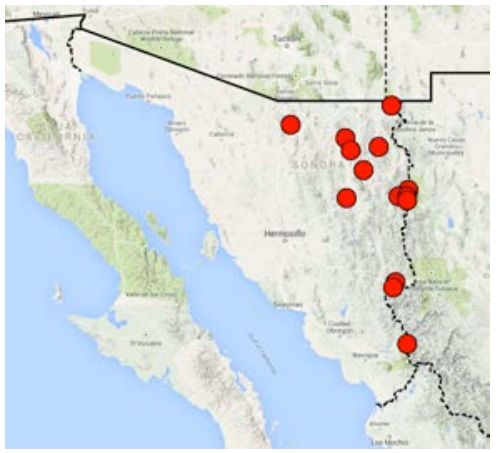

267. Platynus megalops (Bates, 1882)

Number examined: 107.

Municipalities: Aconchi, Álamos, Arizpe, Bacanora, Cananea, Fronteras, Huachinera, Huásabas, Moctezuma, Naco, Nácori Chico, Nacozari de García, Nogales, Ónavas, Yécora.

Habitat: cow pasture; rocky canyon, slope, and mountainside. [at light]

Vegetation: desert grassland; cypress-maple-alder-sycamore riparian forest; oak woodland; pine-oak forest; foothills thornscrub; tropical deciduous forest.

Elevation range: $561-2297 \mathrm{~m}$.

Collection dates: 1982: VIII.03-VIII.04; 1983: VII.11VII.12; 1989: VII.11-VII.13; 1990: VI.30-VII.01, VIII.06-VIII.11; 1993: VII.08-VII.09; 2012: VII.21, IX.02; 2013.IX.08; 2014: VIII.02, VIII.24; 2015: VI.25, VIII.08; 2016.VII.02; 2017: VII.15, VIII.14-VIII.15, IX.16; 2018: VI.28, VIII.05-VIII.09; 2019.VII.06.

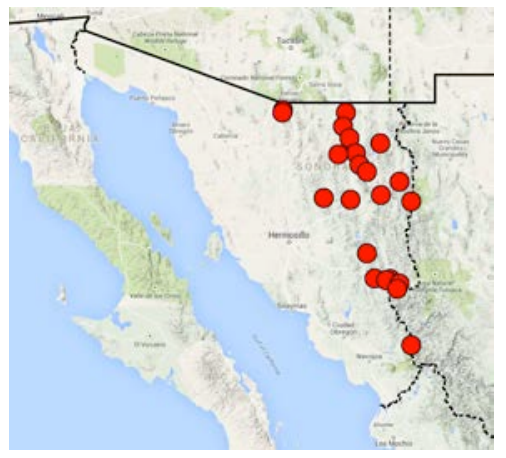

268. Platynus ovatulus (Bates, 1884)

Number examined: 83 .

Municipalities: Agua Prieta, Álamos, Fronteras, Huachinera, Moctezuma, Nácori Chico, Nacozari de García, Ónavas, Yécora.

Habitat: rocky arroyo, slope, and mountainside; stream margin. [at light]

Vegetation: Chihuahuan desertscrub; desert grassland; foothills thornscrub; pine-oak forest; sycamore riparian deciduous forest; tropical deciduous forest.

Elevation range: $500-2266 \mathrm{~m}$.

Collection dates: 1960.VIII.12; 1964.VIII.24; 1982: VIII.02-VIII.04, VIII.09, VIII.11, VIII.19; 1983: VII.04-VII.06; 1987: VII.26-VII.27, VII.30-VII.31;
1989: VII.11-VII.13; 1990: VII.02-VII.03, VIII.06VIII.11; 1993: VII.06-VII.07, VII.09; 2015.VIII.10; 2016: VIII.14, VIII.16; 2018.VIII.06.

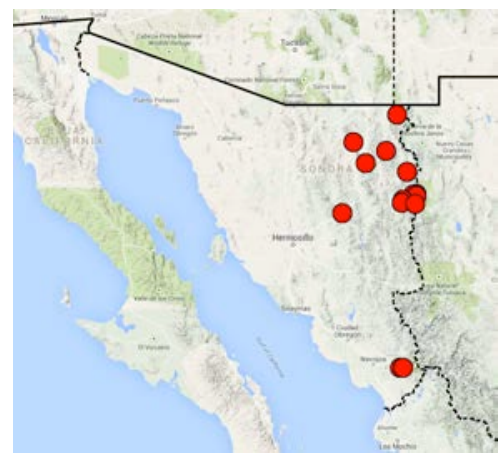

269. Platynus rufiventris (Van Dyke, 1926)

Number examined: 47.

Municipalities: Álamos, Nacozari de García, Yécora

Habitat: rocky ridge. [at light]

Vegetation: oak woodland; pine-oak forest; tropical deciduous forest.

Elevation range: $1547-2467 \mathrm{~m}$.

Collection dates: 1989: VII.11-VII.13; 1990: VI.28VII.03; 2013.VII.17.

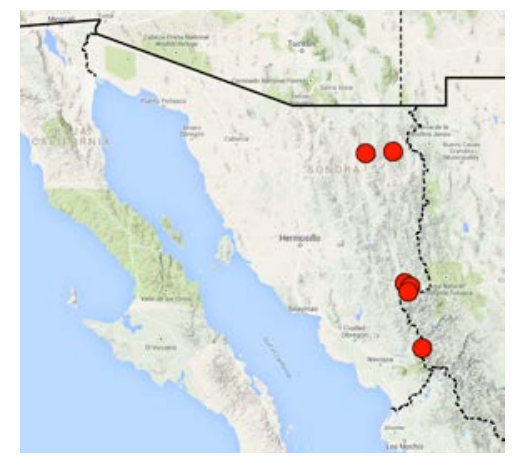

270. Platynus valens (Bates, 1891)

Number examined: 1 .

Municipality: Huachinera.

Habitat: ?

Vegetation: pine-oak forest.

Elevation: $2090 \mathrm{~m}$.

Collection date: 1982: VIII.03-VIII.04.

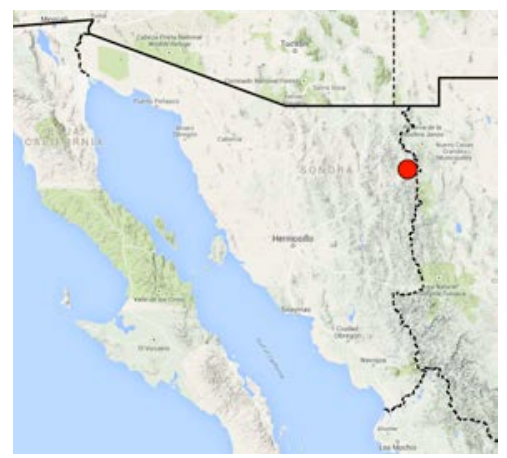




\section{Platynus sp. UASM 19a}

Number examined: 11.

Municipalities: Aconchi, Arizpe, Bacanora, Naco, Moctezuma, Yécora.

Habitat: rocky canyon and slope. [at light]

Vegetation: desert grassland; foothils thornscrub; oak woodland; sycamore riparian deciduous forest.

Elevation range: $944-1680 \mathrm{~m}$.

Collection dates: 1983: VII.11-VII.12; 1990: VI.30-VII.01; 1993: VII.08-VII.09; 2013.VII.02; 2014.VIII.02; 2017. VIII.16; 2019.VII.06.

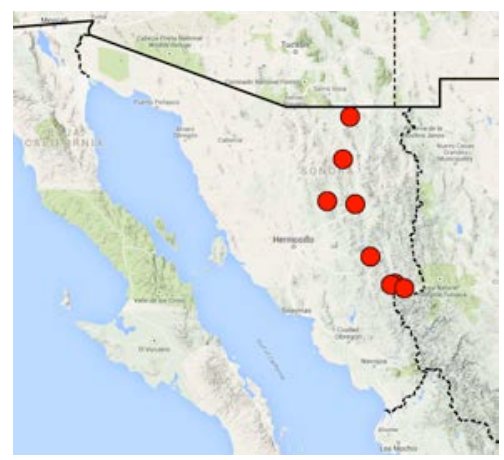

272. Platynus sp. UASM 27a

Number examined: 3 .

Municipality: Yécora.

Habitat: [at light]

Vegetation: oak woodland; pine-oak forest.

Elevation range: $1547-1752 \mathrm{~m}$.

Collection dates: 1990: VI.30-VII.03.

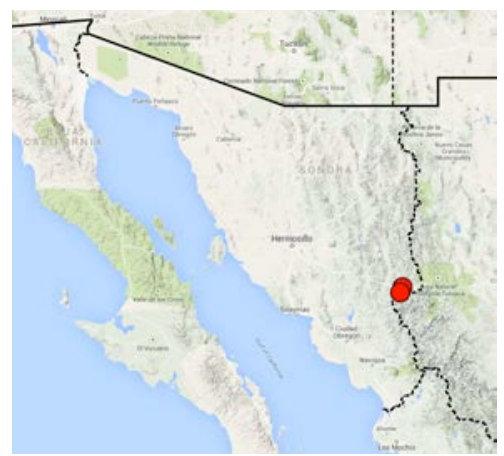

273. Platynus sp. UASM 65b

Number examined: 2.

Municipality: Yécora.

Habitat: [at light]

Vegetation: oak woodland; pine-oak forest.

Elevation: $1311 \mathrm{~m}$.

Collection dates: 1987: VII.28-VII.29.

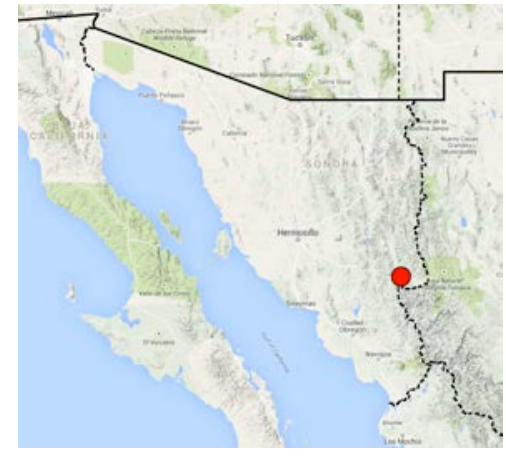

274. Platynus sp. UASM 90

Number examined: 57.

Municipalities: Agua Prieta, Fronteras, Huachinera, Nácori Chico, Nacozari de García, Yécora.

Habitat: rocky arroyo, stream canyon, mountainside, and ridge top; [at light]

Vegetation: Chihuahuan desertscrub; cypress-maple-alder-sycamore riparian forest; oak woodland; pine-oak forest; sycamore riparian deciduous forest.

Elevation range: $1460-2440 \mathrm{~m}$.

Collection dates: 1982: VIII.03-VIII.04, VIII.06-VIII.08, VIII.19; 1983: VII.06-VII.07; 1990: VII.02-VII.03; 2014.VIII.24; 2015: VIII.10, VIII.12; 2018: VIII.07, VIII.09.

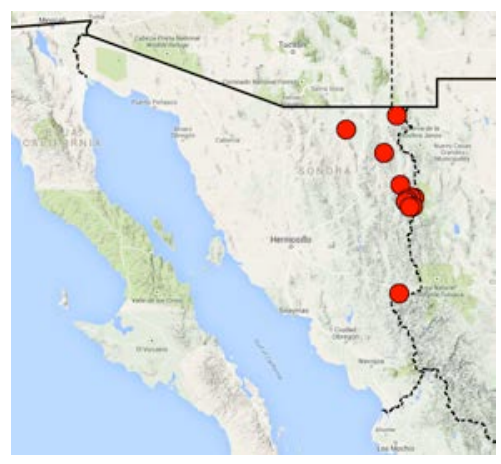

275. Platynus sp. UASM 132

Number examined: 20 .

Municipalities: Nácori Chico, Yécora.

Habitat: arroyo. [at light]

Vegetation: oak woodland; pine-oak forest.

Elevation range: $1547-1950 \mathrm{~m}$.

Collection dates: 1982: VIII.06-VIII.07; 1983.VII.06; 1990: VI.30-VII.03; 1993: VII.06-VII.07, VII.09. 


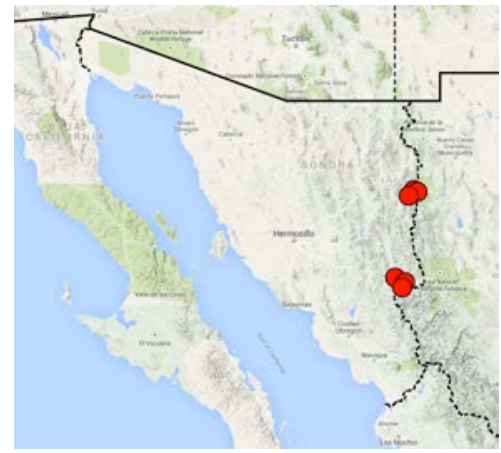

276. Platynus sp. UASM 209a

Number examined: 71 .

Municipalities: Álamos, Nácori Chico, Yécora.

Habitat: rocky stream canyon. [at light]

Vegetation: cypress-maple-alder-sycamore riparian forest; oak forest; oak woodland; pine-oak forest on slope; tropical deciduous forest.

Elevation range: $1311-1950 \mathrm{~m}$.

Collection dates: 1982: VIII.06-VIII.07; 1987: VII.28VII.29; 1989: VII.11-VII.13; 1990: VI.28-VII.03; 1993: VII.06-VII.08; 2018: VI.27, VIII.05, VIII.07.

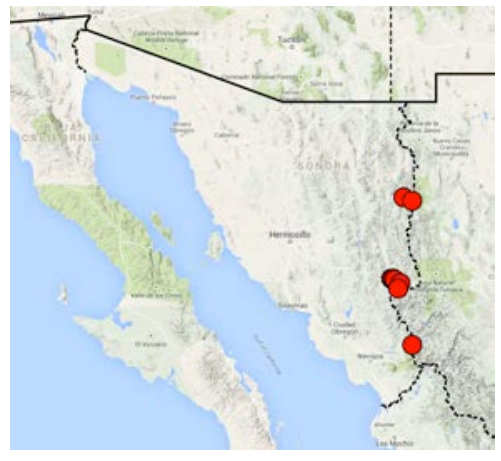

277 Platynus sp. CASC 73

Number examined: 1.

Municipality: Yécora.

Habitat: ?

Vegetation: pine-oak forest.

Elevation: ?

Collection date: 1984.VIII.27.

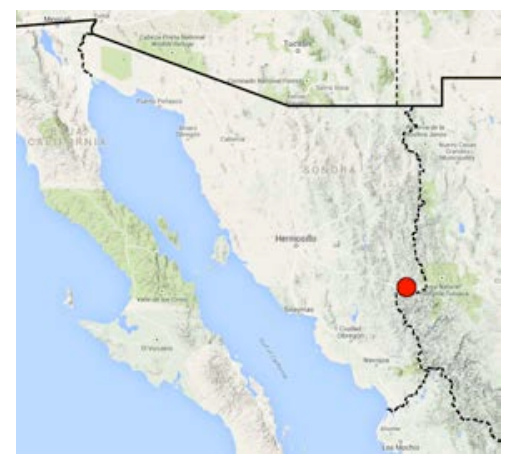

278. Platynus sp. CASC 75

Number examined: 1

Municipality: Álamos.

Habitat: ?

Vegetation: tropical deciduous forest.

Elevation: ?

Collection date: 1935.VII.07.

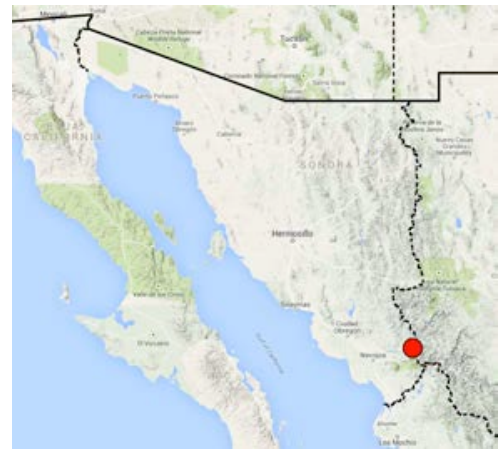

279. Rhadine sp.

Number examined: 2.

Municipality: Huachinera.

Habitat: meadow

Vegetation: pine-oak-forest.

Elevation: $2090 \mathrm{~m}$.

Collection date: 1982.VIII.04.

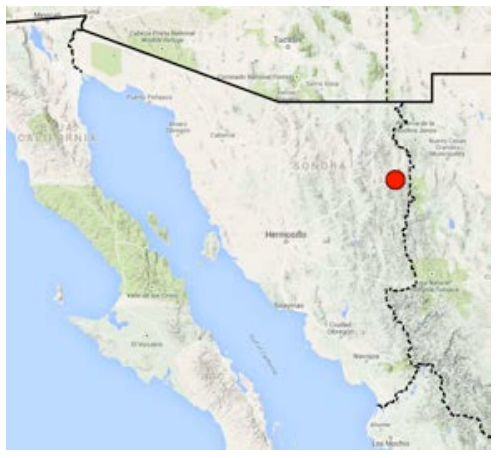

27. Lachnophorini

280. Anchonoderus horni Csiki, 1931

Number examined: 6 .

Municipalities: Álamos, Saric.

Habitat: ?

Vegetation: Sonoran desertscrub; tropical deciduous forest. Elevation: ?

Collection dates: 1929.VII.03; 1960.VIII.12; 1964.VIII.05. 


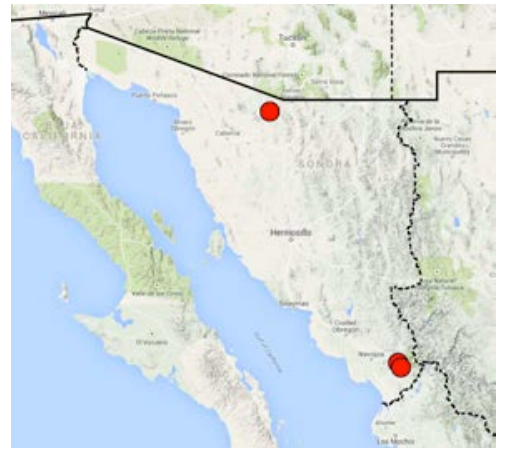

281. Anchonoderus sp.

Number examined: 3 .

Municipality: Álamos.

Habitat: ?

Vegetation: tropical deciduous forest.

Elevation: ?

Collection dates: 1935.VII.15; 1957: VII.28-VII.29; 1965. VII.18.

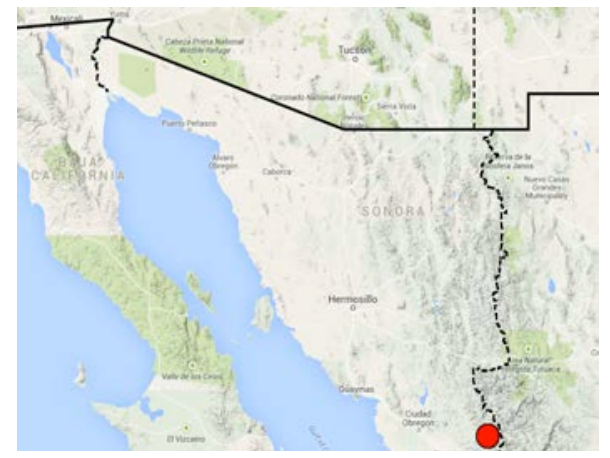

282. Ega laetula LeConte, 1851

Number examined: 3 .

Municipalities: Álamos, Hermosillo.

Habitat: ?

Vegetation: Sonoran desertscrub; tropical deciduous forest.

Elevation: ?

Collection dates: 1897.IV.04; 1935.VII.01; 1955.V.25.

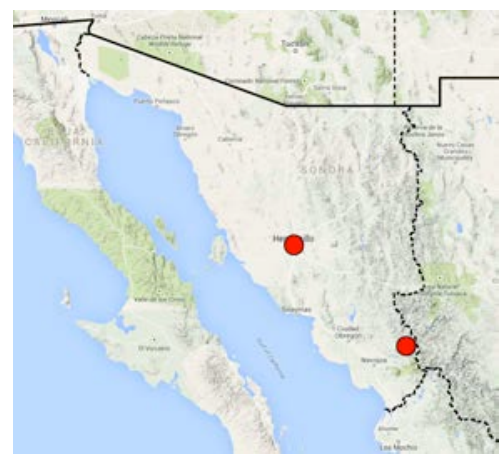

\section{Euphorticus sp.}

Number examined: 71 .

Municipality: Álamos.

Habitat: ?

Vegetation: tropical deciduous forest.

Elevation: ?

Collection dates: 1934.X.03; 1935.VII.15; 1960.VIII.12; 1964.VIII.24.

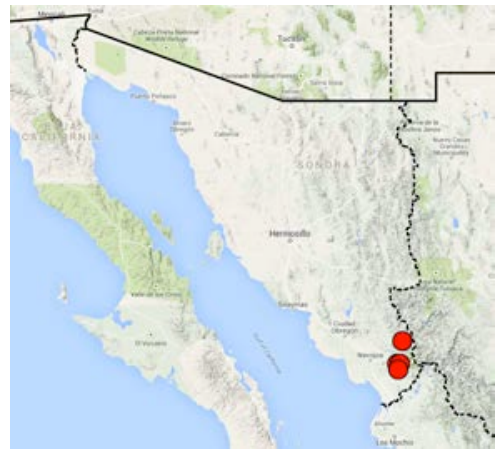

284. Lachnophorus elegantulus Mannerheim, 1843 Number examined: 12.

Municipalities: Álamos, Santa Ana.

Habitat: ?

Vegetation: Sonoran desertscrub; tropical deciduous forest. Elevation: ?

Collection dates: 1934.X.03; 1940.VII.28; 1985.VIII.02.

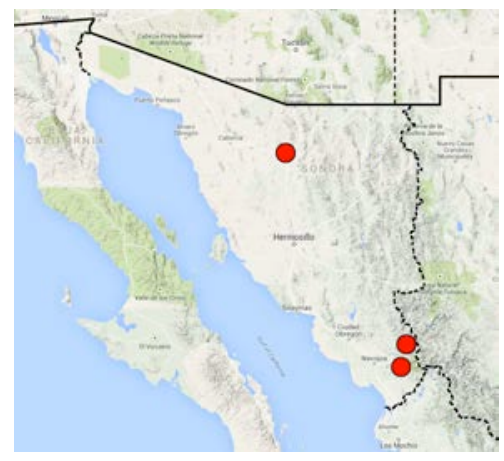

285. Lachnophorus sp.

Number examined: 1.

Municipality: Agua Prieta.

Habitat: riparian.

Vegetation: ?

Elevation: $1350 \mathrm{~m}$.

Collection date: 1982.VIII.20. 


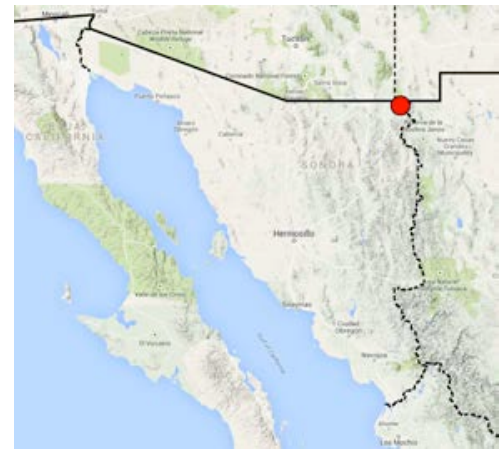

28.Pentagonicini

286. Pentagonica felix Bell, 1987

Number examined: 12.

Municipalities: Álamos, Nácori Chico, Nacozari de García, Yécora.

Habitat: large flat area near ridge top. [at light]

Vegetation: cypress-maple-alder-sycamore riparian forest; oak woodland; pine-oak forest; tropical deciduous forest.

Elevation range: $1547-2261 \mathrm{~m}$.

Collection dates: 1989: VII.11-VII.13; 1990: VI.30VII.03; 2015.VIII.12; 2018: VIII.05, VIII.07, VIII.09.

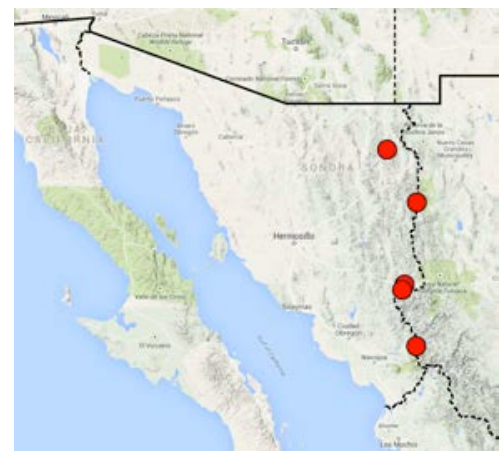

287. Pentagonica sp. UASM-11

Number examined: 65 .

Municipalities: Álamos, Fronteras, Huachinera, Nacozari de García, Ónavas, Santa Ana, Yécora.

Habitat: cow pasture; rocky mountainside. [at light]

Vegetation: Chihuahuan and Sonoran desertscrub; foothills thornscrub; oak woodland; pine-oak forest; tropical deciduous forest.

Elevation range: $710-2090 \mathrm{~m}$.

Collection dates: 1982: VIII.03-VIII.04, VIII.23; 1987: VII.26-VII.27; 1989: VII.11-VII.13; 1990: VI.30VII.03; 1993.VII.09; 2016: VII.02, VIII.14-VIII.16; 2017: VII.14-VII.15, VIII.12, VIII.14-VIII.15.

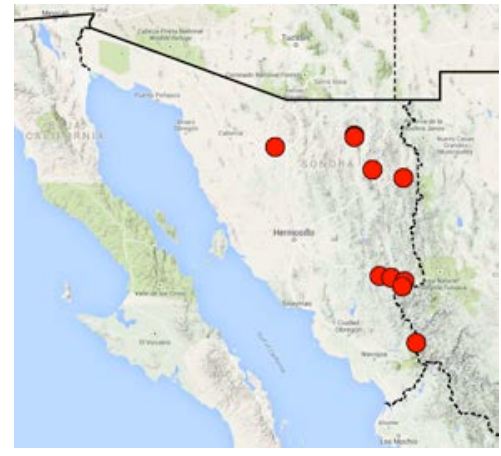

288. Pentagonica sp. CASC 2

Number examined: 1.

Municipality: Álamos.

Habitat: ?

Vegetation: tropical deciduous forest.

Elevation: ?

Collection date: 1935.VII.07.

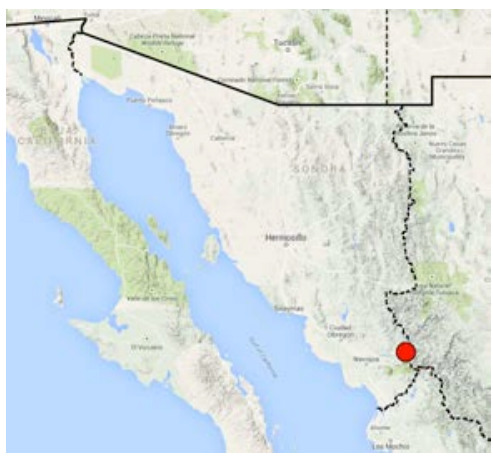

29.Odacanthini

289. Colliuris lengi (Schaeffer, 1910)

Number examined: 1.

Municipality: Nogales.

Habitat: [at light]

Vegetation: desert grassland.

Elevation: ?

Collection date: 1965.VII.30.

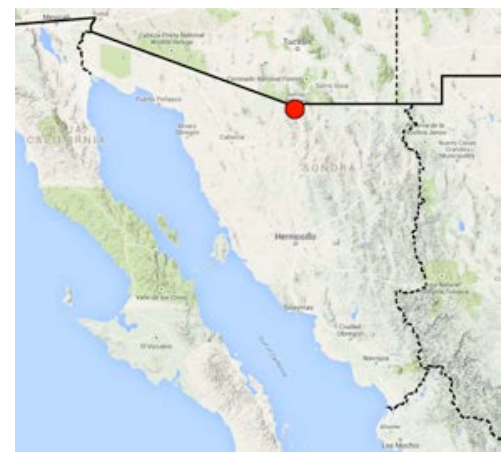

290. Colliuris lioptera (Bates, 1891)

Number examined: 24 .

Municipalities: Aconchi, Álamos, Arizpe, Fronteras, Moctezuma. Nacozari de García, Nogales. 
Habitat: cow pasture; rocky canyon; stream margin. [at light]

Vegetation: Chihuahuan desertscrub; desert grassland; foothills thornscrub; oak woodland; sycamore riparian deciduous forest; tropical deciduous forest.

Elevation range: $500-1577 \mathrm{~m}$.

Collection dates: 1960. VIII. $12 ; 1965$.VII.30; 1982.VIII.02; 1989: VII.11-VII.13; 1993.VII.04; 2013: VII.02, IX.08; 2016.VII.02; 2019.VII.06.

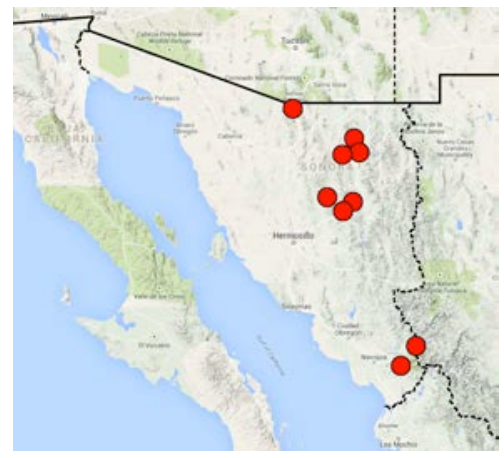

291. Colliuris marginestriata Putzeys, 1845

Number examined: 1.

Municipality: Yécora.

Habitat: ?

Vegetation: pine-oak forest.

Elevation: $1752 \mathrm{~m}$.

Collection date: 1990: VII.02-VII.03.

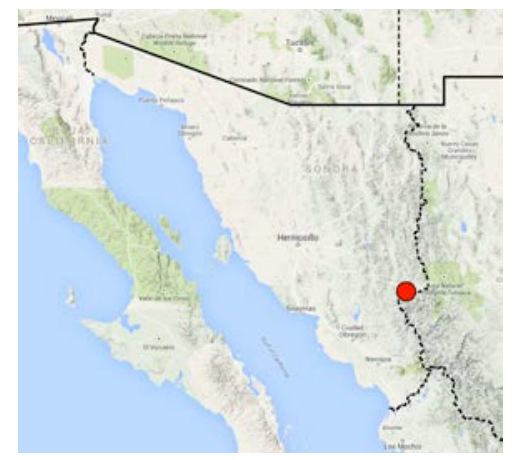

292. Colliuris pensylvanica (Linnaeus, 1758)

Number examined: 123.

Municipalities: Aconchi, Álamos, Bacadéhuachi, Cananea, Carbó, Fronteras, Guaymas, Hermosillo, Huachinera, Moctezuma, Nacozari de García, Navojoa, Nogales, Santa Ana, Santa Cruz, Saric, Soyopa.

Habitat: cow pasture; lights at building at night; PEMEX station; rocky canyon. [at light]

Vegetation: Chihuahuan and Sonoran desertscrub; coastal and foothills thornscrub; desert grassland; oak woodland; sycamore riparian deciduous forest; tropical deciduous forest.

Elevation range: $229-1549 \mathrm{~m}$.

Collection dates: 1953: VII.09-VII.16; 1956.VI.25; 1960: VIII.08, VIII.10, VIII.12; 1962.VII.12; 1963.I.17; 1965:
VII.09, VII.11, VII.30-VII.31; 1967.VII.27; 1982: VIII.01, VIII.03, VIII.05, VIII.23; 1983: VII.11-VII.12; 1989.VII.08; 1993.VII.04; 2012.VIII.09; 2013.VII.02; 2015.VIII.13; 2016.VII.02; 2019: IV.25, VII.23.

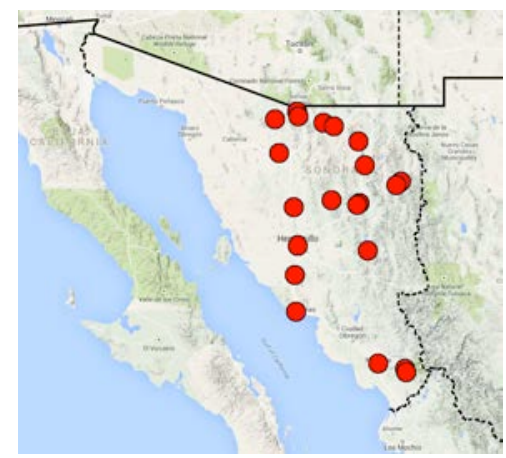

293. Colliuris pilatei (Chaudoir, 1848)

Number examined: 25.

Municipalities: Álamos, Guaymas, Hermosillo, Mazatán, Moctezuma, Navojoa, Ónavas.

Habitat: gentle granitic slopes. [at light]

Vegetation: coastal and foothills thornscrub; oak woodland; Sonoran desertscrub; tropical deciduous forest.

Elevation range: $229-1577 \mathrm{~m}$.

Collection dates: 1935: VI.25, VI.27, VII.09, VII.15; 1953: VII.09-VII.16; 1954.VIII.22; 1958.VIII.06; 1960: VIII.10, VIII.12; 1963.VIII.07; 1964.VIII.05; 1965.VIII.13; 1967.VII.27; 1983: VII.11-VII.12; 1989: VII.11-VII.13; 1990.VIII.06; 2014.VII.28.

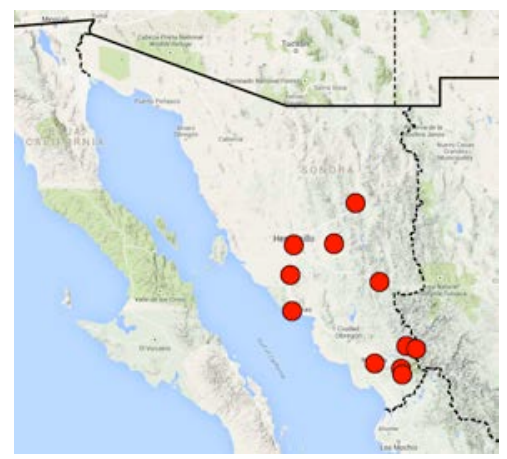

\section{Cyclosomini}

294. Anaulacus mcclevei Ball \& Shpeley, 2002

Number examined: 4.

Municipalities: Álamos, Moctezuma, Navojoa.

Habitat: ex Atta nest; Atta trash pile.

Vegetation: coastal and foothills thornscrub; tropical deciduous forest.

Elevation range: $433-1577 \mathrm{~m}$.

Collection dates: 1962.VII.12; 1980.VII.22; 1989: VII.11VII.13; 2019.VII.24. 


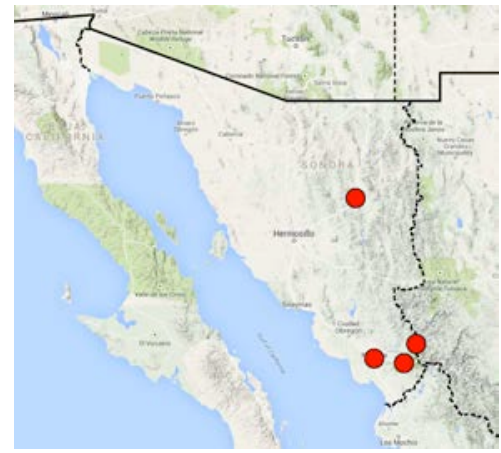

295. Tetragonoderus fasciatus (Haldeman, 1843)

Number examined: 41.

Municipalities: Agua Prieta, Álamos, Carbó, Hermosillo, Ímuris, Moctezuma, Santa Ana.

Habitat: [at light]

Vegetation: Chihuahuan and Sonoran desertscrub; cottonwood-willow riparian deciduous forest; tropical deciduous forest.

Elevation range: $293-1350 \mathrm{~m}$.

Collection dates: 1897.IV.19; 1935.VI.25; 1953: VII.09VII.16; 1965.VII.31; 1982: VIII.19-VIII.20, VIII.23VIII.24; 1986.VIII.29; 1993.VII.04.

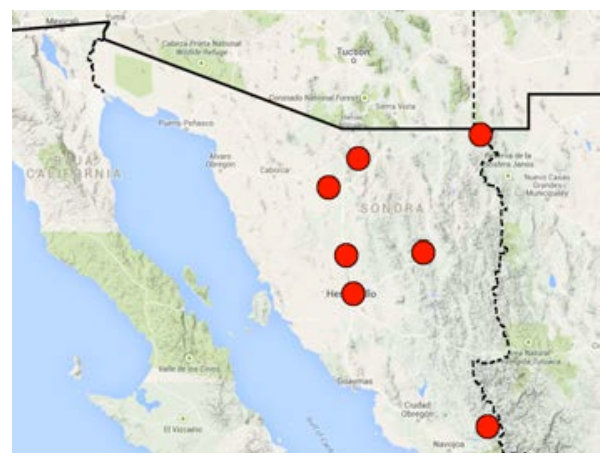

296. Tetragonoderus mexicanus (Chaudoir, 1876)

Number examined: 31 .

Municipalities: Agua Prieta, Moctezuma, Nácori Chico, Nogales, Santa Ana.

Habitat: [at light]

Vegetation: Chihuahuan and Sonoran desertscrub; desert grassland; foothills thornscrub; oak woodland.

Elevation range: $1000-1350 \mathrm{~m}$.

Collection dates: 1982: VIII.19-VIII.20; 1983.VII.03; 1985.VIII.01; 1993.VII.04; 2015: VI.27, VII.12.

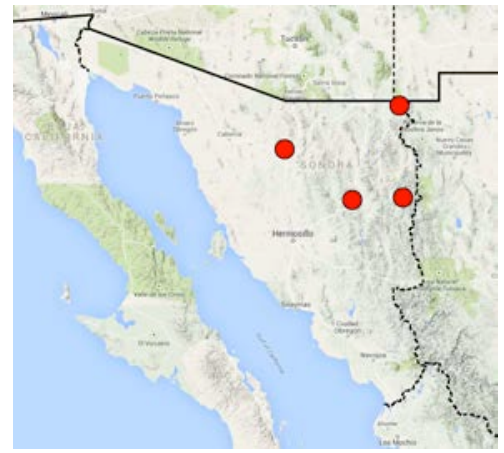

297. Tetragonoderus pallidus G. Horn, 1868

Number examined: 4.

Municipalities: General Plutarco Elias Calles, Pitiquito, Puerto Peñasco.

Habitat: [at light]

Vegetation: Sonoran desertscrub.

Elevation: $152 \mathrm{~m}$.

Collection dates: 1949: III.25, III.31; 1953: VIII.01VIII.15, IX.01-IX.10.

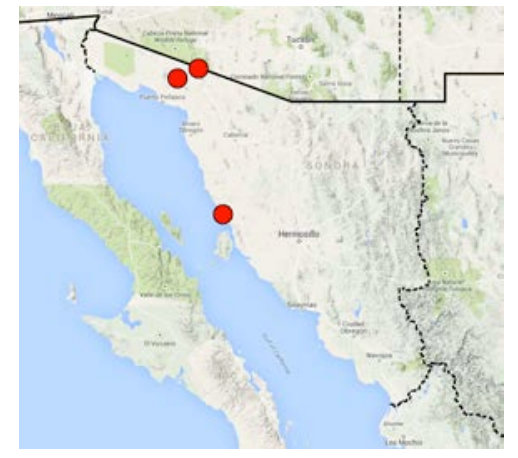

298. Tetragonoderus poecilus Bates, 1883

Number examined: 198.

Municipalities: Agua Prieta, Álamos, Bacadéhuachi, Carbó, Fronteras, Guaymas, Hermosillo, Ímuris, Moctezuma, Nácori Chico, Navojoa, Nogales, Opodepe, Santa Ana.

Habitat: cow pasture; rocky mountainside. [at light]

Vegetation: Chihuahuan and Sonoran desertscrub; cottonwood-willow riparian deciduous forest; desert grassland; foothills thornscrub; oak woodland; tropical deciduous forest.

Elevation range: $293-1420 \mathrm{~m}$.

Collection dates: 1935.VI.25; 1959: VIII.12-VIII.14; 1960: VIII.08, VIII.10, VIII.12; 1963: I.17, II.20, VII.06, VIII.05; 1964.VIII.24; 1966.VIII.29; 1971. VIII.16; 1973.V; 1980.IX.17; 1982: VIII.03, VIII.19VIII.20, VIII.23-VIII.24, 1983: VII.03, VII-11-VII.12; 1986.VIII.29; 1993: VII.04-VII.05; 2013.VIII.09; 2016. VII.02; 2017.VIII.14; 2019.VII.23. 


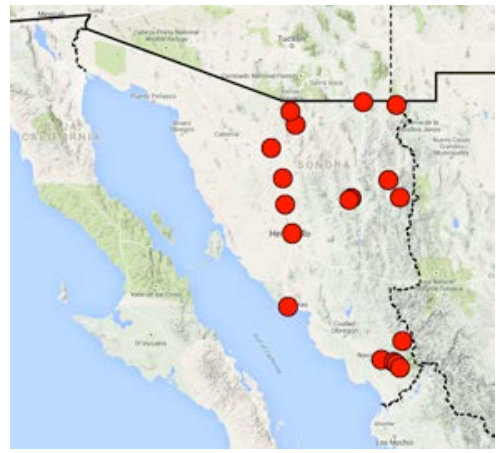

31.Lebiini

299. Agra truquii Chaudoir, 1866

Number examined: 2.

Municipality: Álamos

Habitat: [at light]

Vegetation: tropical deciduous forest.

Elevation: $450 \mathrm{~m}$.

Collection dates: 1964.VII.08; 1977.IX.19.

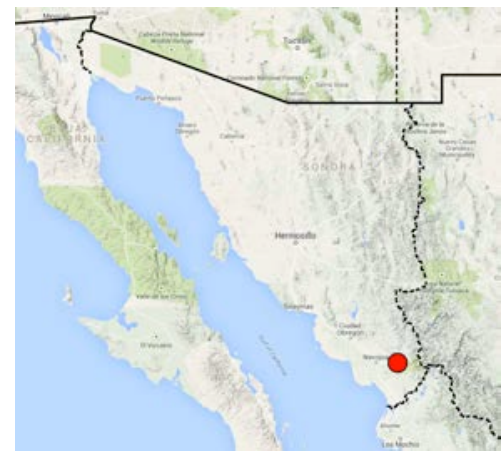

300. Apenes lucidulus (Dejean, 1831)

Number examined: 24.

Municipalities: Álamos, Benjamín Hill, Cananea, Carbó, Fronteras, Hermosillo, Ímuris, Mazatan, Moctezuma, Navojoa, Nogales, Santa Ana, Yécora.

Habitat: urban. [at light]

Vegetation: Chihuahuan and Sonoran desertscrub; coastal and foothills thornscrub; cottonwood-willow riparian deciduous forest; desert grassland; oak woodland; tropical deciduous forest.

Elevation range: $550-1646 \mathrm{~m}$.

Collection dates: 1959: VIII.14-VIII.15; 1960.VIII.08; 1965.VII.30; 1982.VIII.01; 1990: VIII.07-VIII.11; 2012: VII.13, VIII.01, VIII.09, VIII.13; 2013: VIII.04, VIII.09; 2018.III.18.

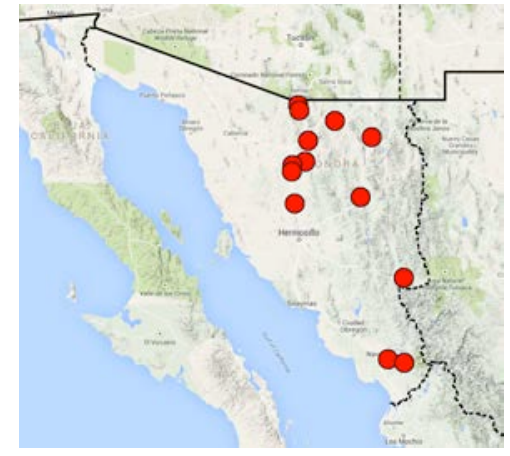

301. Apenes marginalis (Dejean, 1831)

Number examined: 16.

Municipalities: Aconchi, Fronteras, Moctezuma, Nogales, Ónavas, San Felipe de Jesús.

Habitat: cow pasture; rocky canyon [at light].

Vegetation: Chihuahuan desertscrub; desert grassland; foothills thornscrub; oak woodland; sycamore riparian deciduous forest.

Elevation range: $917-1429 \mathrm{~m}$.

Collection dates: 1983: VII.11-VII.12; 1987: VII.26VII.27; 1993.VII.04; 2012.VIII.09; 2013.VII.02; 2016. VII.02; 2019.VIII.04.

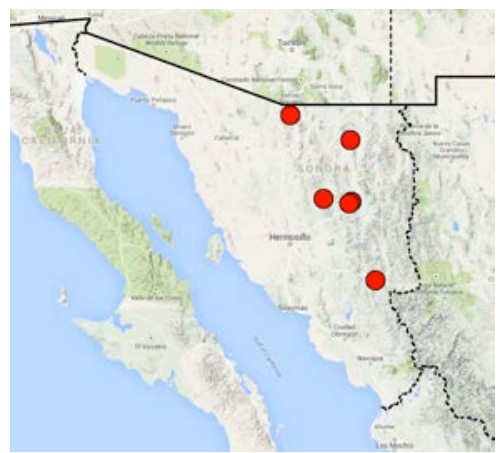

302. Apenes nebulosus LeConte, 1866

Number examined: 14.

Municipalities: Agua Prieta, Álamos, Fronteras, Huásabas, Ímuris, Ónavas, San Felipe de Jesús.

Habitat: rocky arroyo; urban. [at light]

Vegetation: Chihuahuan desertscrub; foothills thornscrub; oak woodland; sycamore riparian deciduous forest; tropical deciduous forest.

Elevation range: $549-1708 \mathrm{~m}$.

Collection dates: 1935.VII.15; 1957: VII.28-VII.29; 1960. VIII.12; 1964. VIII.08; 1972.X; 1982: VIII.19-VIII.20, VIII.24; 1987: VII.26-VII.27; 2016: V.05, VIII.14, VIII.16; 2019.VIII.04. 


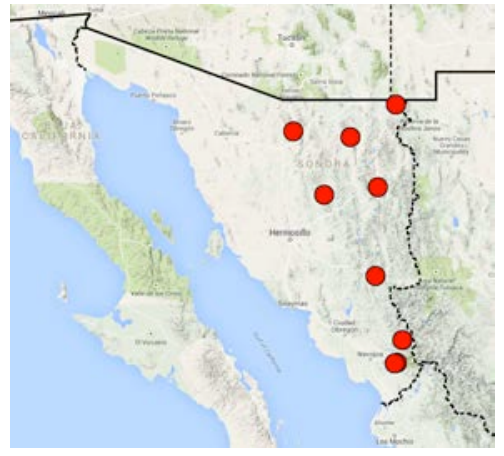

303. Apenes marmorata complex sp.

Number examined: 1 .

Municipality: Guaymas.

Habitat: ?

Vegetation: Sonoran desertscrub.

Elevation: ?

Collection date: 1960.VIII.10.

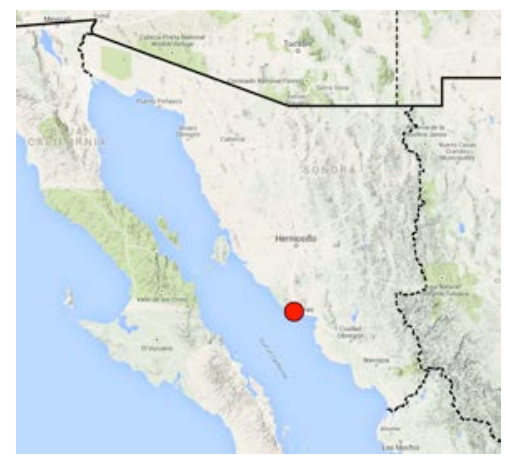

304. Apristus pugetanus Casey, 1920

Number examined: 4.

Municipalities: Álamos, Guaymas, Hermosillo, San Javier.

Habitat: ?

Vegetation: Sonoran desertscrub; tropical deciduous forst.

Elevation: ?

Collection dates: 1897.IV.19; 1929.IV.03; 1963.II.26; 1973.V.

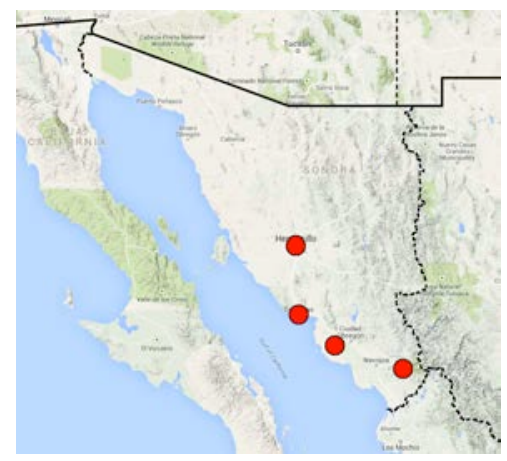

305. Apristus sp.

Number examined: 1.

Municiplaity: Huachinera.

Habitat: ?
Vegetation: desert grassland.

Elevation: $1150 \mathrm{~m}$.

Collection date: 1982.VIII.05

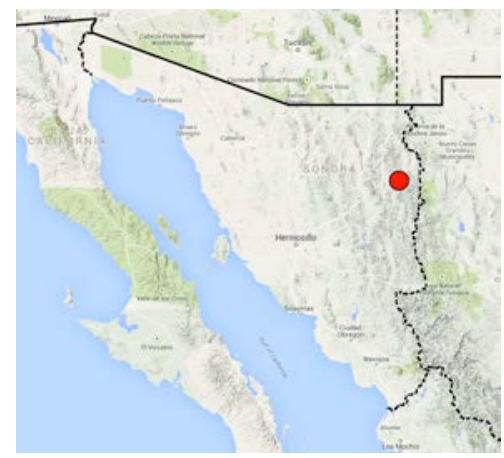

306. Apristus sp. CASC 1

Number examined: 2

Municipality: Hermosillo.

Habitat: ?

Vegetation: Sonoran desertscrub.

Elevation:?

Collection dates: 1953: VII.09-VII.16.

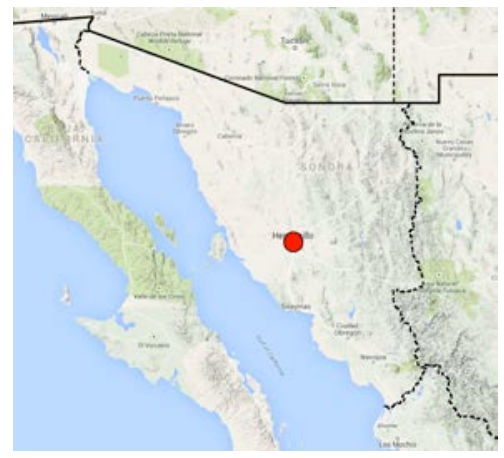

307. Axinopalpus biplagiatus (Dejean, 1825)

Number examined: 4.

Municipality: Hermosillo.

Habitat: ?

Vegetation: Sonoran desertscrub.

Elevation: ?

Collection dates: 1953: VII.09-VII.16.

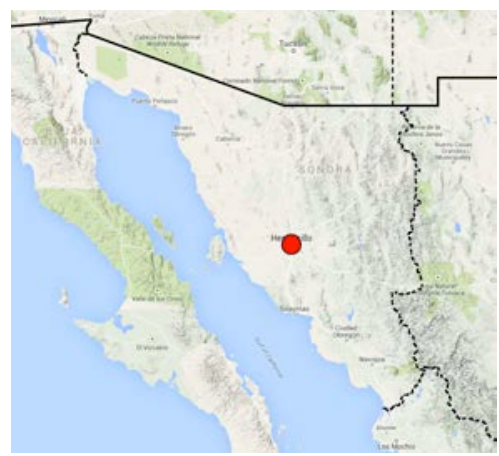


308. Axinopalpus fusciceps LeConte, 1851

Number examined: 4.

Municipalities: Guaymas, Hermosillo.

Habitat: ?

Vegetation: Sonoran desertscrub.

Elevation:?

Collection dates: 1953: VII.09-VII.16; 1973.V.

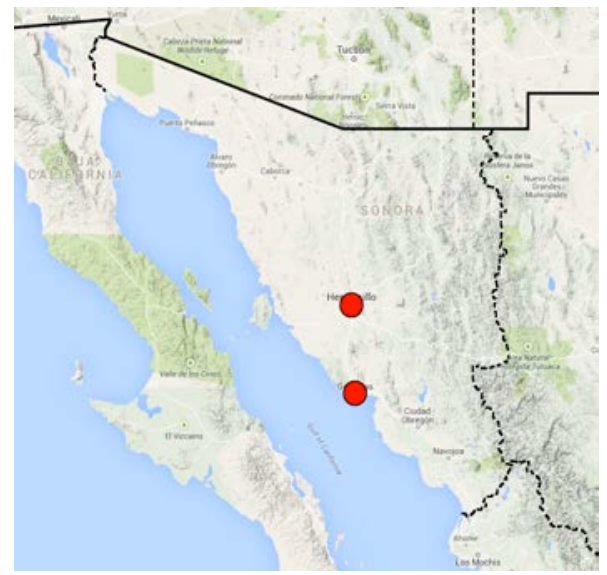

309. Axinopalpus sp.

Number examined: 1 .

Municipality: Guaymas.

Habitat: Sonoran desertscrub.

Vegetation:?

Elevation:?

Collection date: 1973 . V.

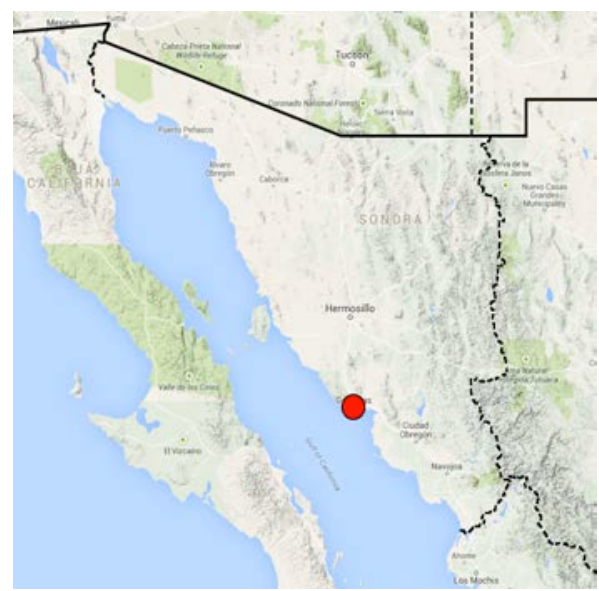

310. Calleida brunnea Dejean, 1831

Number examined: 2 .

Municipalities: Aconchi, Nacozari de García.

Habitat: rocky arroyo and canyon.

Vegetation: oak woodland; sycamore riparian deciduous forest.

Elevation range: 1301 - $1388 \mathrm{~m}$.

Collection dates: 2013.VII.02; 2017.VIII.14.

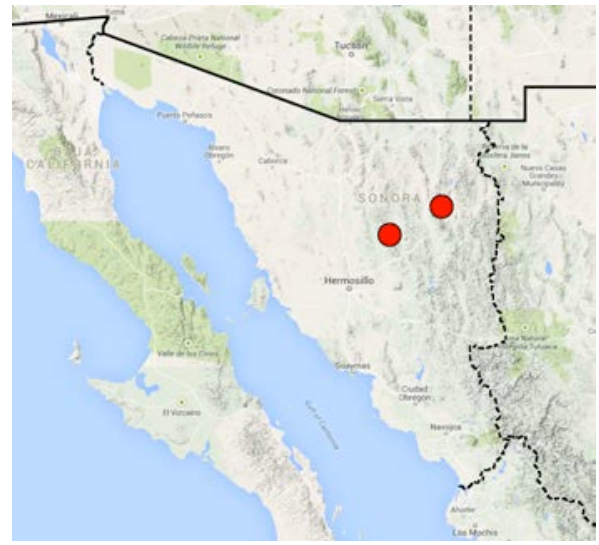

311. Calleida cordicollis Putzeys, 1845

Number examined: 194.

Municipalities: Aconchi, Álamos, Bacanora, Cananea, Carbó, Fronteras, Granados; Guaymas, Hermosillo, Moctezuma, Nácori Chico, Nacozari de García, Navojoa, Ónavas, San Felipe de Jesús, Soyopa, Yécora.

Habitat: cow pasture; rocky canyon, slope, and hillside; stock pond. [at light]

Vegetation: Chihuahuan and Sonoran desertscrub; coastal and foothills thornscrub; desert grassland; sycamore riparian deciduous forest; oak woodland; tropical deciduous forest.

Elevation range: $277-1577 \mathrm{~m}$.

Collection dates: 1935: VII.07, VII.09, VII.15; 1940. VII.28; 1953: VII.09-VII.16; 1957: VII.28-VII.29, IX.07; 1960: VIII.08, VIII.11-VIII.12; 1963.VIII.07; 1964.VIII.05; 1965: VI.11, VII.07, VII.11, VII.15, VII.30; 1967.VII.27; 1973.X.11; 1982: VIII.01, VIII.09; 1987: VII.26-VII.27, VII.30-VII.31; 1989: VII.08, VII.11-VII.13; 1990.VIII.08; 1993: VII.04, VII.09; 2012.VII.07; 2014: VIII.02, IX.14; 2015.VIII.08; 2016.VII.02; 2017.VII.22; 2018.VIII.11; 2019: VII.22, VIII.04-VIII.05, VIII.09.

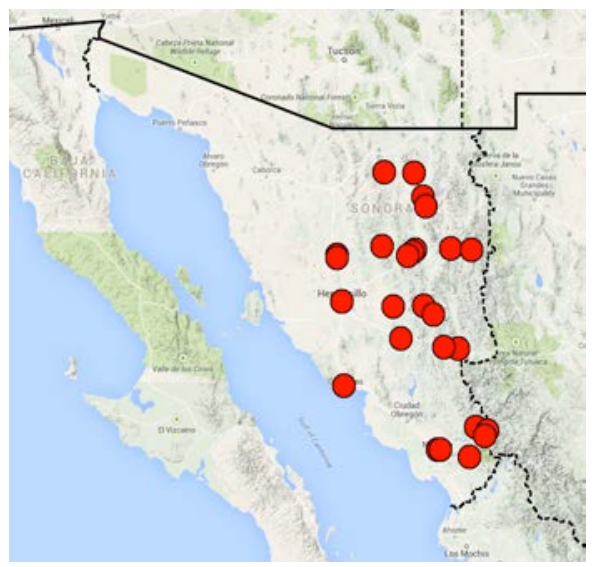

312. Calleida cyanippe Bates, 1883

Number examined: 7.

Municipalities: Álamos, Bacanora, Nacozari de García, Ónavas, Soyopa. 
Habitat: rocky slope and mountainside; [at light].

Vegetation: foothills thornscrub; oak woodland; tropical deciduous forest.

Elevation: $180-1687 \mathrm{~m}$.

Collection dates: 1957: VII.28-VII.29; 1990.VIII.08; 2012.VIII.06; 2014.VIII.02; 2017.VIII.14.

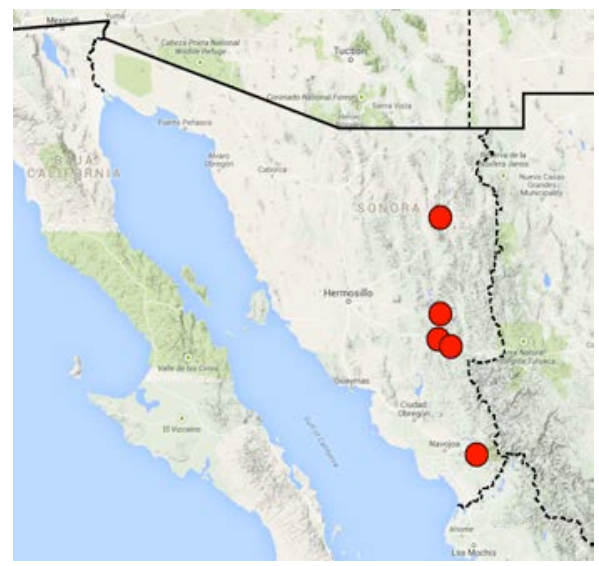

313. Calleida platynoides G. Horn, 1882

Number examined: 2 .

Municipality: Opodepe.

Habitat: ?

Vegetation: Sonoran desertscrub.

Elevation:?

Collection date: 1963.VII.06.

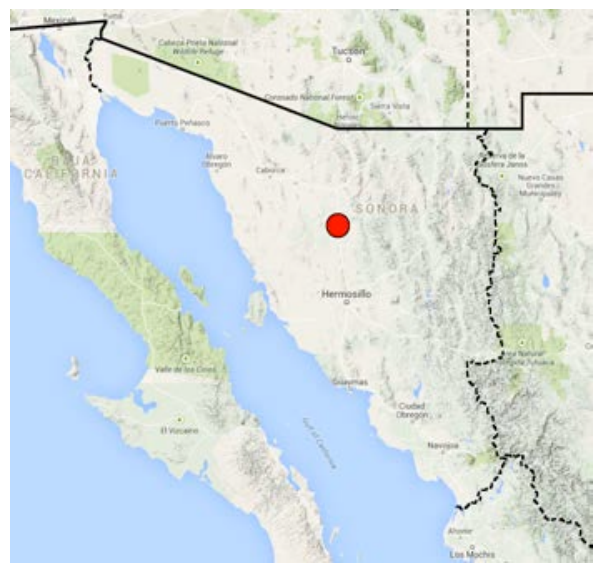

314. Calleida punctulata Chaudoir, 1848

Number examined: 64 .

Municipalities: Álamos, Bacanora, Cucurpe, Ónavas, Yécora.

Habitat: rocky canyon and slope. [at light]

Vegetation: foothills thornscrub; oak woodland; tropical deciduous forest.

Elevation range: $876-1387 \mathrm{~m}$.

Collection dates: 1934.X.25; 1935: I.03, VII.07; 1957: VII.28-VII.29; 1958.VIII.06; 1960: VII.21, VIII.12; 1964: VII.24, VIII.08; 1987: VII.26-27, VII.30-VII.31; 1993.VII.09; 2014: VIII.02, VIII.29; 2019: VII.22, VII.25.

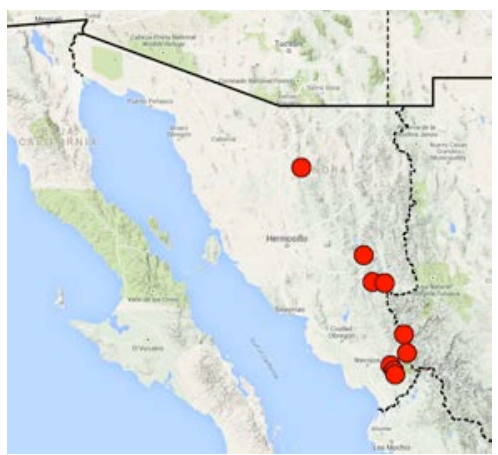

\section{Calleida sp. CASC 2}

Number examined: 16.

Municipalities: Álamos, Guaymas.

Habitat: ?

Vegetation: Sonoran desertscrub; tropical deciduous forest. Elevation:?

Collection dates: 1935: VII.07, VII.09; 1960.VIII.10.

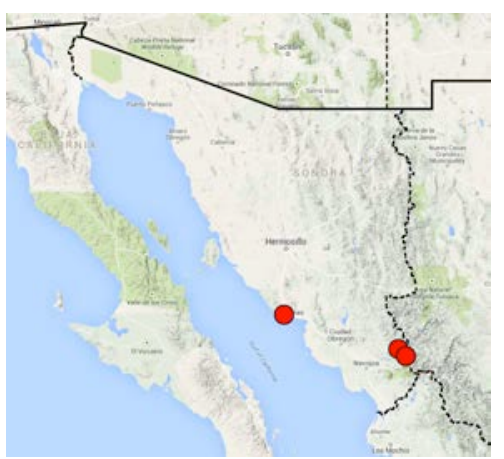

316. Calleida sp. CASC 9

Number examined: 1 .

Municipality: Álamos.

Habitat: ?

Vegetation: tropical deciduous forest.

Elevation: $400 \mathrm{~m}$

Collection date: 1935.VIII.21.

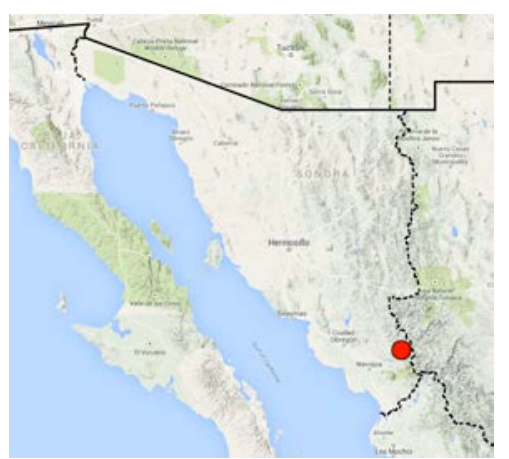


Number examined: 1 .

\section{Calleida sp. CASC 19}

Muicipality: Álamos.

Habitat: ?

Vegetation: tropical deciduous forest.

Elevation: $400 \mathrm{~m}$

Collection date: 1960.VII.21.

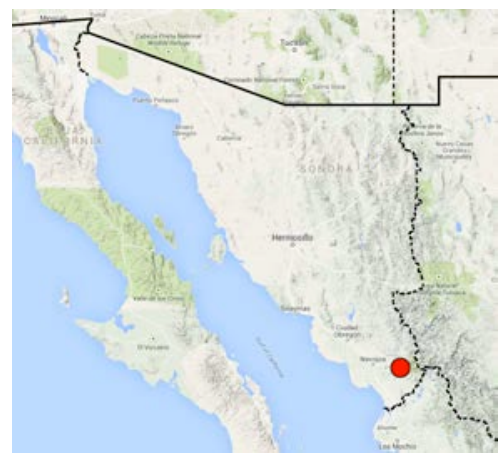

318. Coptodera brunnea Shpeley \& Ball, 1993

Number examined: 11.

Municipalities: Agua Prieta, Arizpe, Fronteras, Nacozari de García.

Habitat: arroyo; rocky mountainside. [at light]

Vegetation: desert grassland; oak woodland; sycamore riparian deciduous forest.

Elevation range: 1275 - 1698 m.

Collection dates: 2016:VIII.16; 2017: IV.23, VII.15; 2019. VII.06.

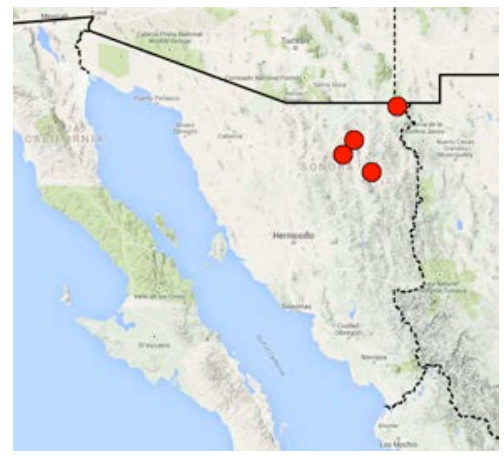

319. Coptodera nitidula (Buquet, 1834)

Number examined: 9.

Municipalities: Agua Prieta, Nacozari de García, Yécora.

Habitat: rocky mountainside. [at light]

Vegetation: cottonwood-willow riparian deciduous forest; oak woodland.

Elevation range: $1291-1687 \mathrm{~m}$.

Collection dates: 1987: VII.28-VII.29; 1990: VI.30VII.01; 2017: IV.23, VII.15.

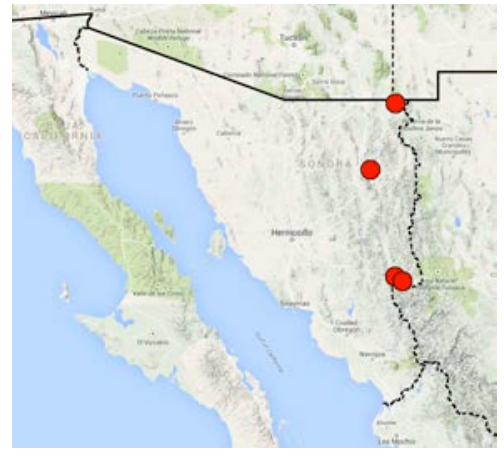

320. Cymindis arizonensis Schaeffer, 1910

Number examined: 4.

Municipalities: Cananea, Nacozari de Garcia, Nogales, Santa Cruz.

Habitat: rocky slope.

Vegetation: desert grassland; oak woodland; pine-oak forest.

Elevation range: 1217 - $2422 \mathrm{~m}$.

Collection dates: 1982.VIII.11; 2013.VIII.13; 2019: IV.24IV.25.

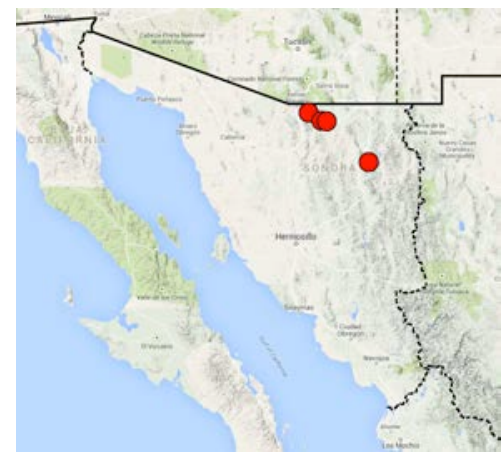

321. Cymindis punctifera punctifera (LeConte, 1884)

Number examined: 70 .

Municipalities: Álamos, Arizpe, Fronteras, Guaymas, Moctezuma, Naco, Nacozari de García, Ónavas, Yécora.

Habitat: rocky mountainside. [at light]

Vegetation: Chihuahuan desertscrub; desert grassland; foothills thornscrub; oak woodland; pine-oak forest; tropial deciduous forest.

Elevation range: $435-1900 \mathrm{~m}$.

Collection dates: 1935.VII.15; 1960.VIII.10; 1983: VII.11VII.12; 1987: VII.26-VII.31; 1993: VII.04, VII.08VII.09; 2015: VIII.10, VIII.12; 2016: VIII.14, VIII.16; 2017: VII.15, VIII.12, VIII.15-VIII.16; 2019: VII.06, VII.22, VII.25. 


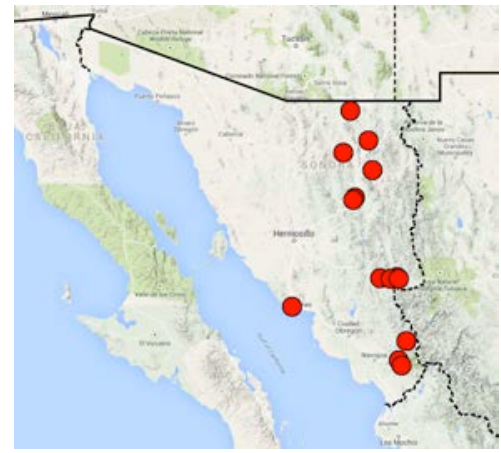

322. Cymindis punctigera punctigera (LeConte, 1851) Number examined: 43.

Municipalities: Agua Prieta, Álamos, Cajeme, Cananea, Hermosillo, Ímuris, Moctezuma, Nácori Chico, Opodepe, Yécora.

Habitat: gentle rocky slope; river bottom; urban. [at light] Vegetation: Chihuahuan and Sonoran desertscrub; coastal and foothills thornscrub; cottonwood-willow riparian forest; oak woodland; pine-oak forest; tropical deciduous forest.

Elevation range: $744-2134 \mathrm{~m}$.

Collection dates: 1921.VII.03; 1961: V.16-V.23; 1964. VIII.25; 1970.IX.17; 1982: VI.09-VI.11, VIII.01, VIII.19-VIII.21; 1992.VII.09; 1994.IX.11; 2012.II.06; 2013.VIII.04; 2014.IX.20; 2017.IV.21.

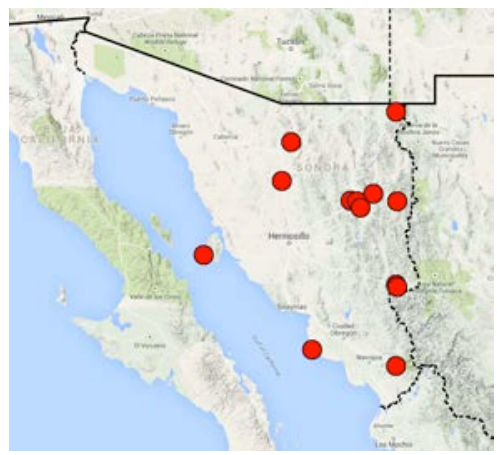

323. Cymindis uniseriata Bates, 1884

Number examined: 24.

Municipalities: Huachinera, Nácori Chico, Nacozari de García.

Habitat: rocky arroyo, slope, and ridge top; [at light]

Vegetation: pine-oak forest.

Elevation range: $2090-2440 \mathrm{~m}$.

Collection dates: 1982: VIII.03-VIII.04; 1983.VII.07;

2015.VIII.10-VIII.12; 2017.IV.21.

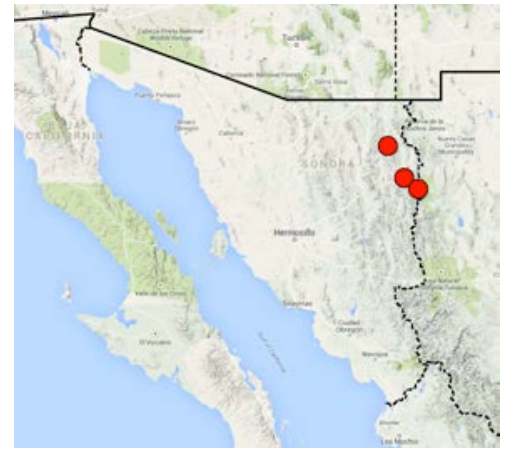

324. Cymindis sp. son-1

Number examined: 1 .

Municipality: Nacozari de García.

Habitat: ?

Vegetation: desert grassland; oak woodland.

Elevation: $1400 \mathrm{~m}$.

Collection date: 1982.VIII.11.

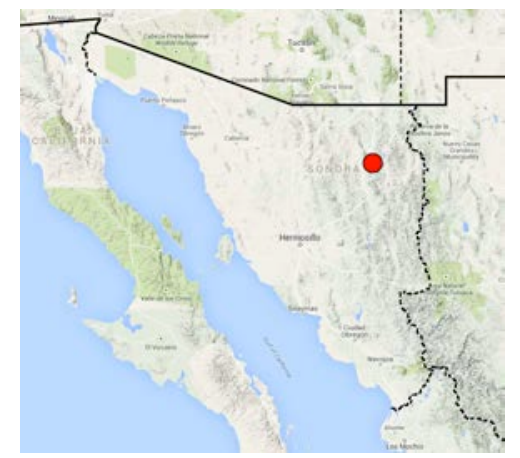

325. Cymindis sp. son-2

Number examined: 24.

Municipalities: Álamos, Huachinera, Nácori Chico, Yécora.

Habitat: [at light]

Vegetation: oak woodland; pine-oak forest; tropical deciduous forest.

Elevation range: $1311-2200 \mathrm{~m}$.

Collection dates: 1961: V.20-V.22; 1981.VI.25; 1982: VIII.03-VIII.04, VIII.06-VIII.07; 1983: VII.05-VII.06; 1987: VII.28-VII.29; 1989: VII.11-VII.12; 1990: VI.38VII.03, VIII.07-VIII.11.

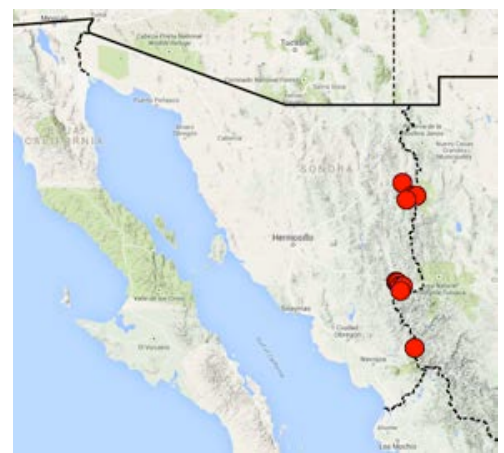




\section{Cymindis sp. son-3}

Number examined: 21.

Municipalities: Huachinera, Nácori Chico, Yécora.

Habitat: [at light]

Vegetation: oak woodland; pine-oak forest.

Elevation range: $1547-2200 \mathrm{~m}$.

Collection dates: 1961: V.20-V.22; 1981.VI.25; 1982:

VIII.03-VIII.04, VIII.06-VIII.07; 1983: VII.05-VII.06; 1990: VI.28-VII.03.

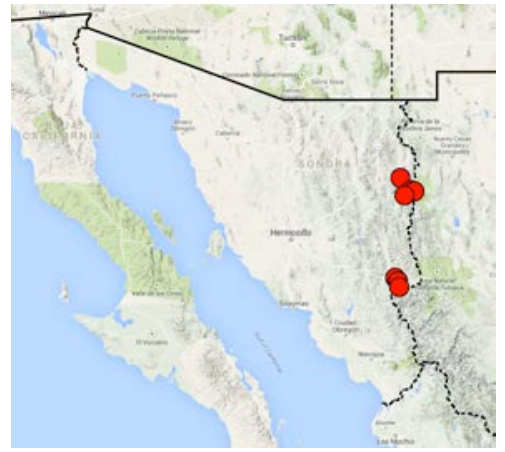

327. Dromius piceus Dejean, 1831

Number examined: 22.

Municipalities: Fronteras, Nácori Chico, Nacozari de García.

Habitat: rocky arroyo, canyon, mountainside, ridge top. [at light]

Vegetation: cypress-maple-alder-sycamore riparian forest; oak woodland; pine-oak forest; sycamore riparian deciduous forest.

Elevation range: $1681-2297 \mathrm{~m}$.

Collection dates: 2015: VI.25, VIII.10, VIII.12; 2016: VIII.14-VIII.15; 2017: VII.15, VIII.12, VIII.14-VIII.15; 2018: VIII.08-VIII.09.

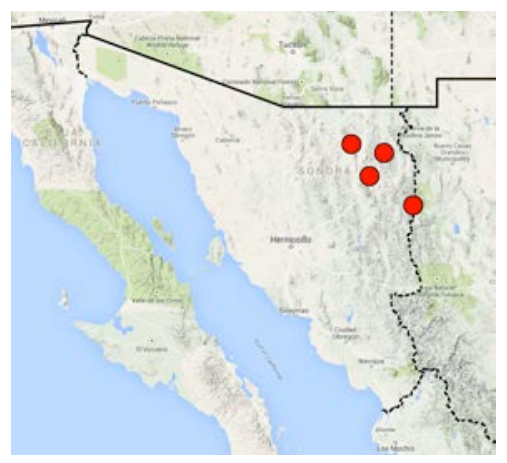

328. Euproctinus abjectus (Bates, 1883)

Number examined: 13.

Municipalities: Álamos, Yécora.

Habitat: [at light]

Vegetation: pine-oak forest; tropical deciduous forest.

Elevation range: $518-1646 \mathrm{~m}$.

Collection dates: 1987: VII.30-VII.31; 1989: VII.11-

VII.13; 1990: VI.28-VI.29; 2019.VII.23.

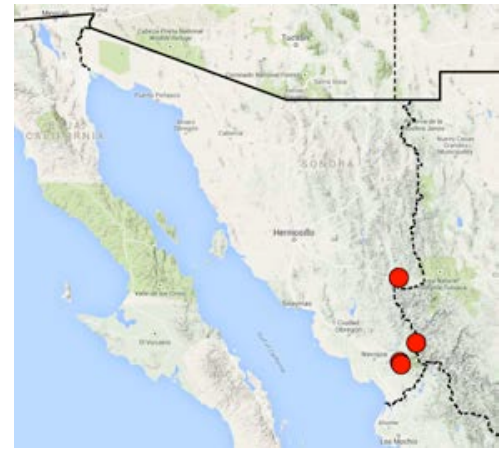

329. Euproctinus balli Shpeley, 1986

Number examined: 25 .

Municipalities: Nácori Chico, Nacozari de García, Yécora.

Habitat: rocky mountainside; [at light]

Vegetation: oak woodland; pine-oak forest.

Elevation range: 1547 - $1950 \mathrm{~m}$.

Collection dates: 1983.VII.06; 1990: VI.28-VII.01; 2017. VII.15.

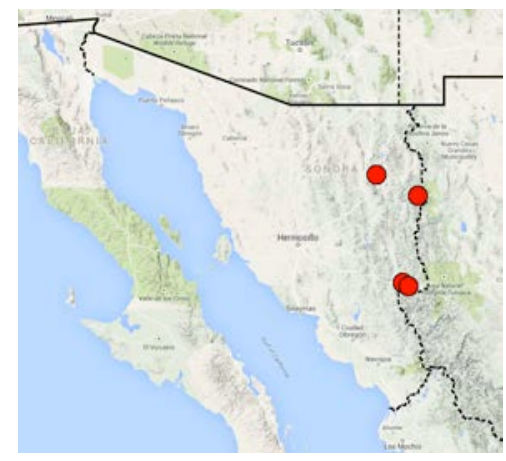

330. Euproctinus sigillatus (Bates, 1883)

Number examined: 11.

Municipalities: Alamos, Yécora.

Habitat: [at light]

Vegetation: oak woodland; pine-oak forest.

Elevation range: $1547-1752 \mathrm{~m}$.

Collection dates: 1989: VII.11-VII.13; 1990: VI.28-VII.03, VIII.07-VIII.11.

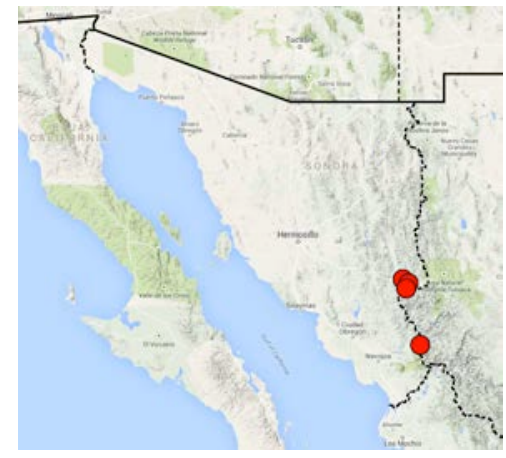


331. Lebia (Chelonodema) balli (Reichardt, 1972)

Number examined: 32 .

Municipalities: Aconchi, Álamos, Baviácora, Moctezuma, Nácori Chico, Nacozari de García, Ónavas, Yécora.

Habitat: rocky canyon and mountainside. [at light]

Vegetation: foothills thornscrub; oak woodland; pine-oak forest; sycamore riparian deciduous forest; tropical deciduous forest.

Elevation range: $917-1687 \mathrm{~m}$.

Collection dates: 1959.VIII.14; 1980.IX.24; 1982.VIII.05; 1987: VII.26-VII.29; 1993.VII.09; 2012.IX.02; 2015. VIII.08; 2017: VII.15, VIII.14; 2018.VIII.09.

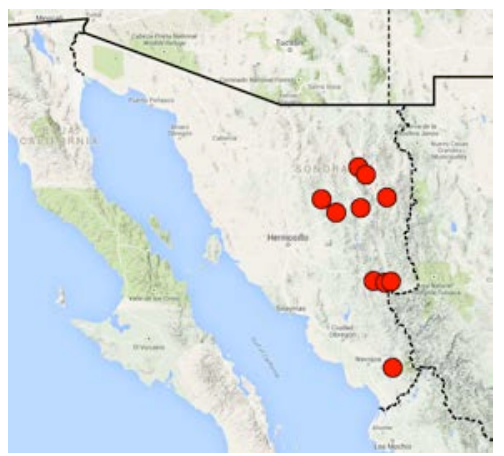

332. Lebia (Chelonodema) quadrinotata (Chevrolat, 1835)

Number examined: 44 .

Municipalities: Álamos, Moctezuma, Nácori Chico, Ónavas, Yécora.

Habitat: rocky river canyon. [at light]

Vegetation: foothills thornscrub; oak woodland; pine-oak forest; sycamore riparian deciduous forest; tropical deciduous forest.

Elevation range: $518-1680 \mathrm{~m}$.

Collection dates: 1934.X.25; 1935.VIII.25; 1984: VIII.02VIII.04; 1987: VII.30-VII.31; 1989: VII.11-VII.13; 1990: VI.28-VI.29; 1993: VII.08-VII.09; 2018.VIII.05.

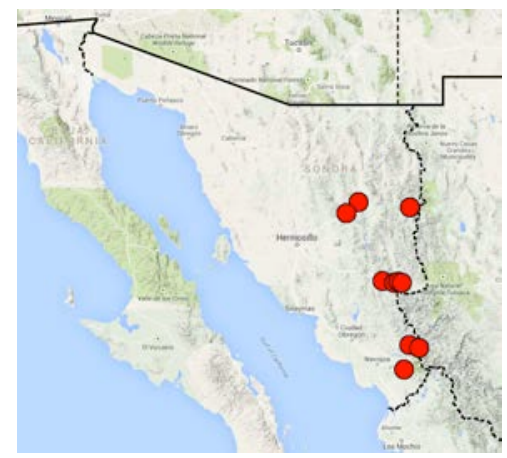

333. Lebia (Lebia) abdita Madge, 1967

Number examined: 11.

Municpalities: Agua Prieta, Santa Ana.

Habitat: [at light]

Vegetation: cottonwood-willow riparian deciduous forest; oak woodland; Sonoran desertscrub.
Elevation range: $710-1350 \mathrm{~m}$.

Collection dates: 1982: VIII.19-VIII.20, VIII.23; 2017. IV.23.

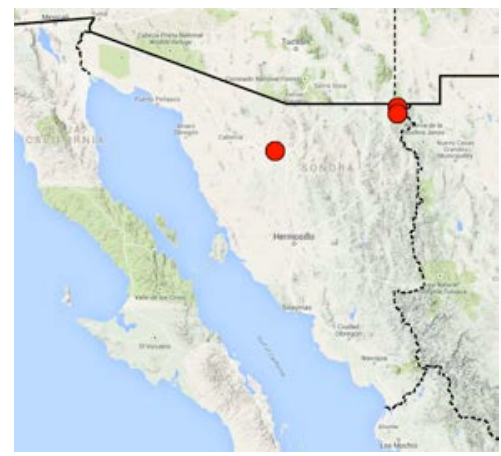

334. Lebia (Lebia) analis Dejean, 1825

Number examined: 183.

Municipalities: Aconchi, Agua Prieta, Álamos, Arizpe, Bacanora, Baviácora, Fronteras, Hermosillo, Huachinera, Ímuris, Magdalena de Kino, Moctezuma, Nácori Chico, Nacozari de García, Nogales, Ónavas, Opodepe, San Felipe de Jesús, Santa Ana, Soyopa, Ures, Yécora.

Habitat: cow pasture; rocky arroyo, stream canyon, slopes, mountainside, and ridge top; [at light]

Vegetation: cypress-maple-alder-sycamore riparian forest; foothills thornscrub; oak woodland; pine-oak forest; Sonoran desertscrub; sycamore riparian deciduous forest; tropical deciduous forest.

Elevation range: $229-2261 \mathrm{~m}$.

Collection dates: 1935.VII.15; 1953: VIII.15-VIII.20; 1958: VII.07-VII.11; 1960.VIII.12; 1963.VII.06, VIII.05; 1965: VI.11, VII; 1967.VII.27; 1980.IX.24; 1982: VIII.01, VIII.03-VIII.04, VIII.06-VIII.07, VIII.23; 1983: VII.03, VII.11-VII.12; 1987: VII.26VII.27, VII.30-VII.31; 1989: VII.11-VII.13; 1990: VI.28-VII.03; 1993: VII.04, VII.09; 2012: VII.21, IX.02; 2013: VII.02, VII.16, VIII.12, IX.07; 2014. VIII.02; 2015: VI.24, VIII.12; 2016: VII.02-VII.03, VIII.15-VIII.16; 2017: I.11, IV.21; 2018.VI.27; 2019: VII.22, VII.25, VIII.04-VIII.05.

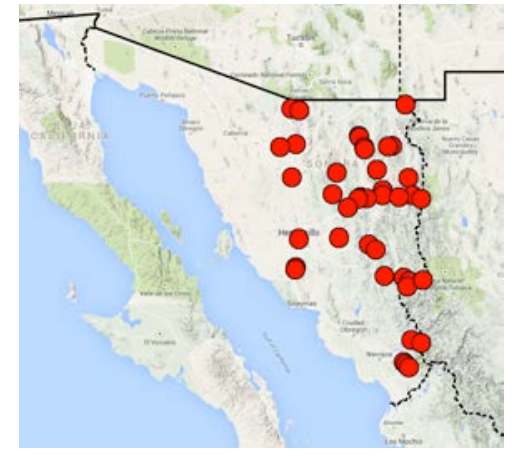

335. Lebia (Lebia) chalybe Bates, 1883 
Number examined: 7 .

Municipality: Álamos.

Habitat: in bromeliads [at light]

Vegetation: tropical deciduous forest.

Elevation range: $518-1577 \mathrm{~m}$.

Collection dates: 1935.VII.07; 1957: VII.28-VII.29; 1987:VII.30-VII.31; 1989: VII.11-VII.13.

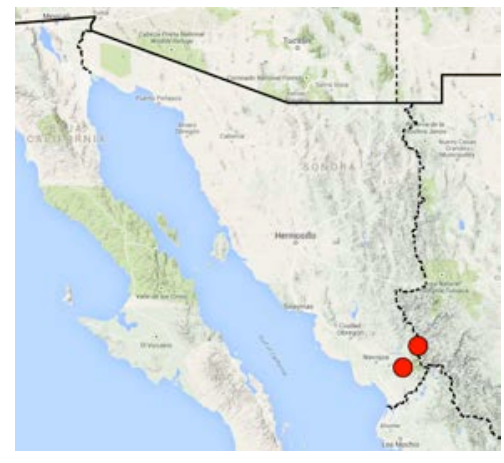

336. Lebia (Lebia) cymindoides Bates, 1883

Number examined: 150 .

Municipalities: Agua Prieta, Arizpe, Cananea, Fronteras, Huachinera, Magdalena de Kino, Naco, Nácori Chico, Nacozari de García, Nogales.

Habitat: arroyo; cow pasture; rocky stream canyon, slope, mountainside, and ridge top. [at light]

Vegetation: cypress-maple-alder-sycamore riparian forest; desert grassland; oak woodland; pine-oak forest; Sonoran desertsrub;

Elevation range: $1161-2467 \mathrm{~m}$.

Collection dates: 1982: VIII.03-VIII.04, VIII.06-VIII.07, VIII.12-VIII.20; 1983: VII.04, VII.06; 2012.VII.13; 2013: VII-16-VII.17, VIII.02; 2014.VIII.24; 2015: VIII.12, IX.13; 2016: VII.02-VII.03, VIII.13-VIII.16; 2018: VI.27, VIII.06, VIII.09; 2019.VII.06.

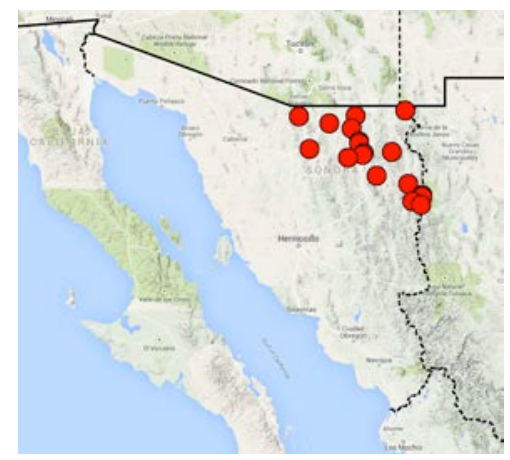

Number examined: 1.

Municipality: Nacozari de García.

Habitat: rocky mountainside. [at light]

Vegetation: oak woodland.

Elevation: $1681 \mathrm{~m}$.

Collection date: 2017.VIII.12.

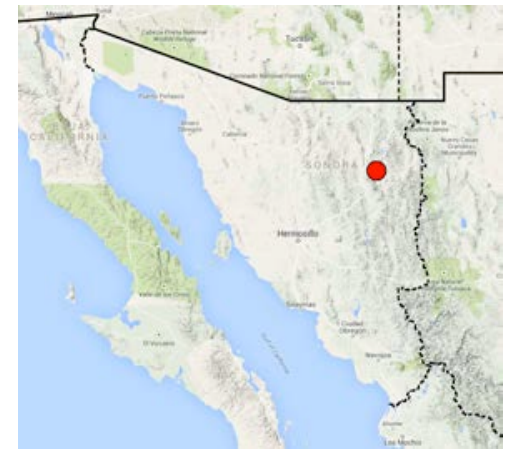

338. Lebia (Lebia) guttula LeConte, 1851

Number examined: 8 .

Municipalities: Guaymas, Puerto Peñasco.

Habitat:

Vegetation: Sonoran desertscrub.

Elevation:

Collection dates: 1960.VIII.10; 1962.IV.15.

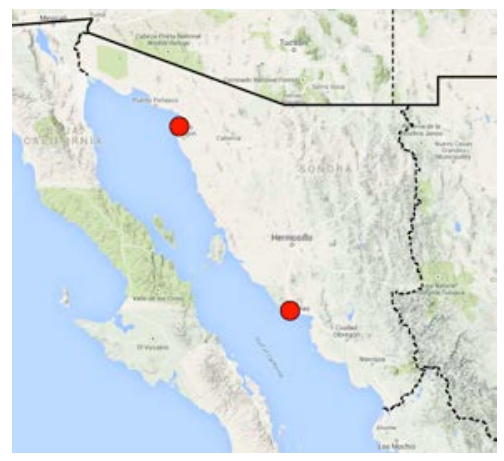

339. Lebia (Lebia) histrionica Bates, 1883

Number examined: 9.

Municipalities: Aconchi, Bacadéhuachi, Cajeme, Cananea, Nacozari de García, Navojoa, Yécora.

Habitat: flat area in canyon bottom; rocky canyon; rocky hillside. [at light]

Vegetation: coastal thornscrub; desert grassland; oak woodland; pine-oak forest; sycamore riparian deciduous forest.

Elevation range: $1216-1752 \mathrm{~m}$.

Collection dates: 1954.VIII.22; 1982.VIII.03; 1990: VII.02-VII.03; 2012.IX.02; 2013: VII.16, IX.07; 2014. IX.14; 2015.VI.24. 


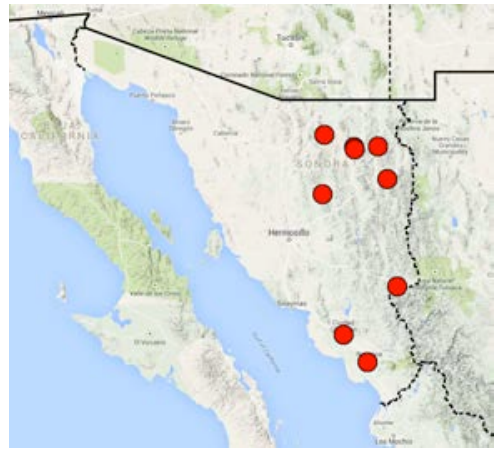

340. Lebia (Lebia) miranda G. Horn, 1872

Number examined: 41.

Municipalities: Arizpe, Bacadéhuachi, Guaymas, Mazatán, Moctezuma, Nácori Chico, Nogales, Opodepe, San Felipe de Jesús, Santa Ana.

Habitat: meadow; rocky canyon. [at light]

Vegetation: desert grassland; foothills thornscrub; oak woodland; Sonoran desertscrub.

Elevation range: $550-1420 \mathrm{~m}$,

Collection dates: 1960.VIII.10; 1963.VIII.06; 1982: VIII.01, VIII.03, VIII.23; 1983.VII.03 2012.VIII.13; 2017.I.11; 2018.III.18; 2019: VIII.04 - VIII.05.

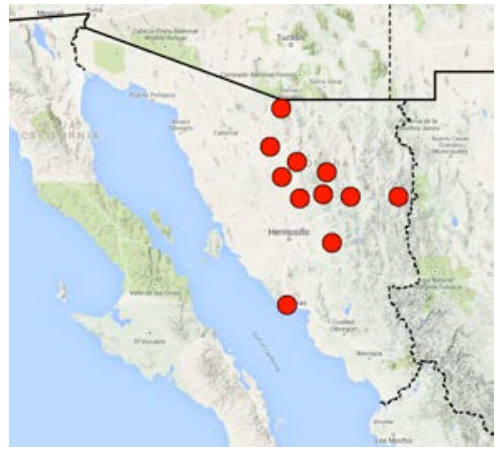

341. Lebia (Lebia) scalpta Bates, 1883

Number examined: 1.

Municipality: Bacanora.

Habitat: rocky slope.

Vegetation: oak woodland.

Elevation: $1387 \mathrm{~m}$.

Collection date: 2014-08-02

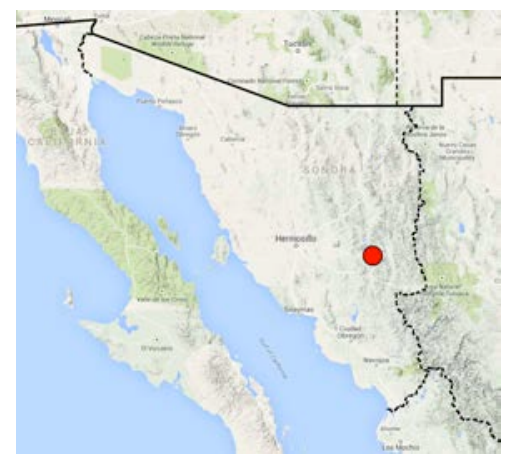

342. Lebia (Lebia) subrugosa Chaudoir, 1871

Number examined: 1.

Municipality: Agua Prieta.

Habitat: ?

Vegetation: cottonwood-willow riparian deciduous forest; oak woodland.

Elevation: $1291 \mathrm{~m}$.

Collection date: 2017.IV.23.

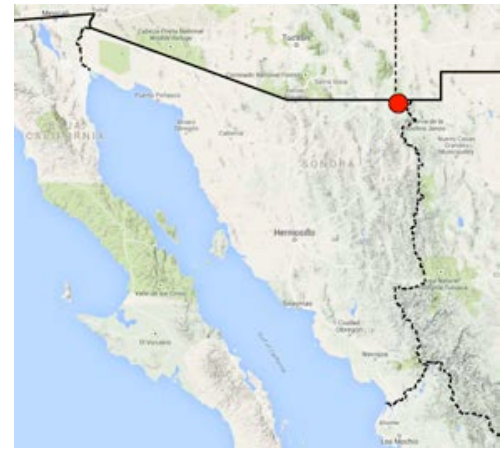

343. Lebia (Lebia) tuckeri (Casey, 1920)

Number examined: 211.

Municipalities: Agua Prieta, Álamos, Bacanora, Huachinera, Magdalena de Kino, Moctezuma, Naco, Nácori Chico, Nacozari de García, Ónavas, San Felipe de Jesús; Santa Ana, Santa Cruz, Ures, Yécora.

Habitat: rocky arroyo, stream canyon, slope, mountainside; [at light]

Vegetation: cypress-maple-alder-sycamore riparian forest; desert grassland; foothills thornscrub; oak woodland; pine-oak forest; Sonoran desertscrub; tropical deciduous forest.

Elevation range: $930-2090 \mathrm{~m}$.

Collection dates: 1982: VIII.01, VIII.03-VIII.05, VIII.19VIII.20, VIII.23; 1987: VII.26-VII.27, VII.30-VII.31; 1989: VII.11-VII.13; 1990: VI.28-VI.29, VII.02-VII.02; 1993: VII.04, VII.06-VII.09; 2014: VII.28, VIII.02; 2015.IX.13; 2017: IV.21, VII.15, VIII.12, VIII.14VIII.15, IX.16; 2018.VI.27; 2019: IV.25, VIII.05.

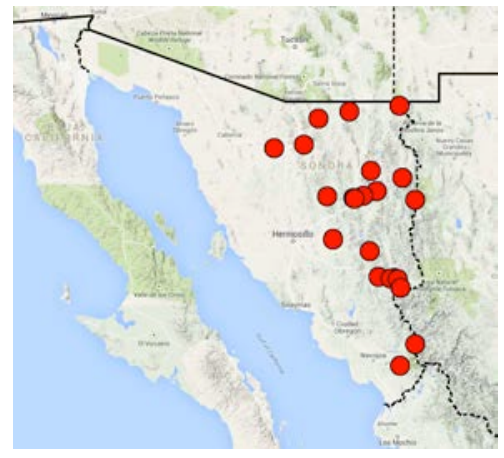


344. Lebia (Lebia) viridis Say, 1823

Number examined: 23.

Municipalities: Agua Prieta, Cananea, Moctezuma, Nácori Chico, Nacozari de García.

Habitat: arroyo; litter; river valley; rocky slope. [at light]

Vegetation: desert grassland; foothills thornscrub; oak woodland; pine-oak forest.

Elevation range: $1000-2300 \mathrm{~m}$.

Collection dates: 1982: IX.19-08.20; 1983: VII.04, VII.06; 1993.VII.04; 2014.IX.14; 2015.VIII.11; 2017: IV.22, IV.24.

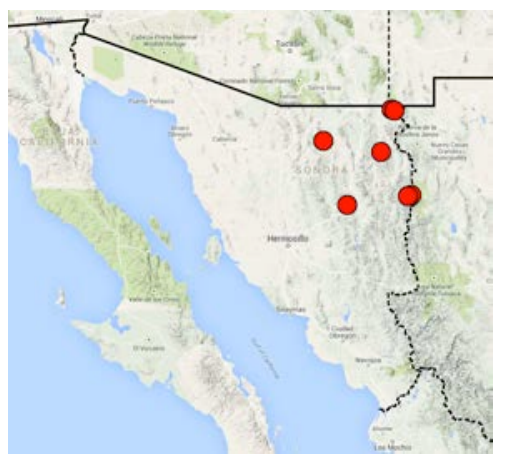

345. Lebia (Lebia) sp. nr. chalybe Bates, 1883 (= sp. CASC 113)

Number examined: 3 .

Municipality: Álamos.

Habitat: ?

Vegetation: tropical deciduous forest.

Elevation: $400 \mathrm{~m}$

Collection date: 1935.VII.07.

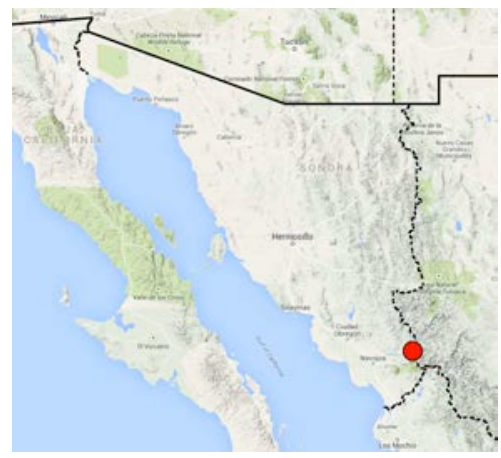

346. Lebia (Lebia) sp. nr. charilla Bates, 1883 (= sp. CASC 124)

Number examined: 1 .

Municipality: Álamos.

Habitat: ?

Vegetation: tropical deciduous forest.

Elevation: $400 \mathrm{~m}$

Collection date: 1960.VIII.12.

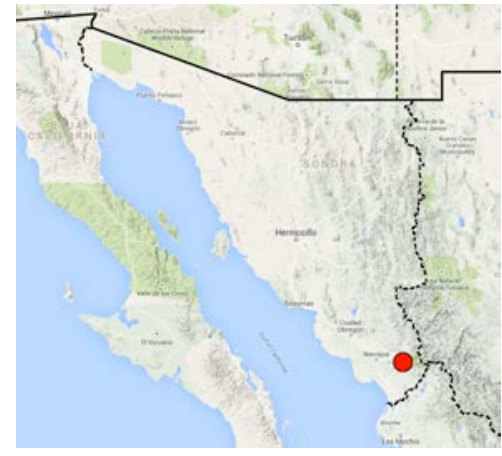

347. Lebia (Lebia) sp. son-5

Number examined: 2 .

Municipality: Agua Prieta.

Habitat: [at light]

Vegetation: cottonwood-willow riparian deciduous forest; desert grassland; oak woodland.

Elevation: $1350 \mathrm{~m}$.

Collection dates: 1982: VIII.19-VIII.20.

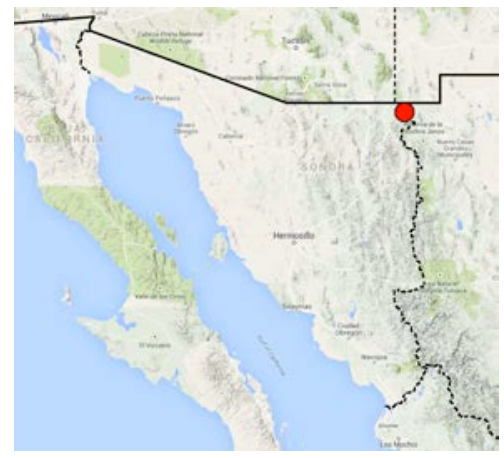

348. Lebia (Lebia) sp. UASM-4

Number examined: 1 .

Municipality: Nácori Chico.

Habitat: rocky stream canyon. [at light]

Vegetation: cypress-maple-alder-sycamore riparian forest; pine-oak forest.

Elevation: $1648 \mathrm{~m}$

Collection date: 2018.VIII.09.

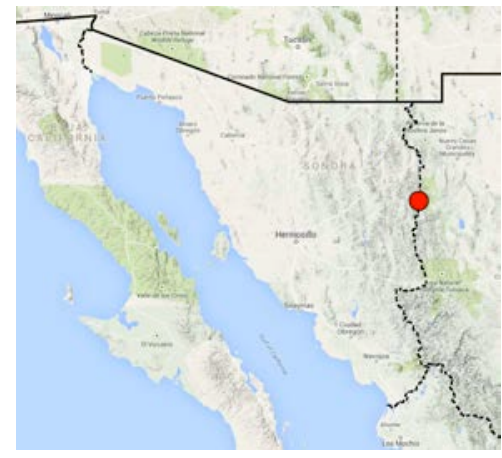


Number examined: 5 .

Municipalities: Fronteras, Moctezuma, Naco, Nacozari de García.

Habitat: rocky mountainside. [at light]

Vegetation: desert grassland; foothills thornscrub; oak woodland.

Elevation range: $1000-1707 \mathrm{~m}$.

Collection dates: 1993.VII.04; 2016: VIII.15-VIII.16; 2017: VII.15, IX.16.

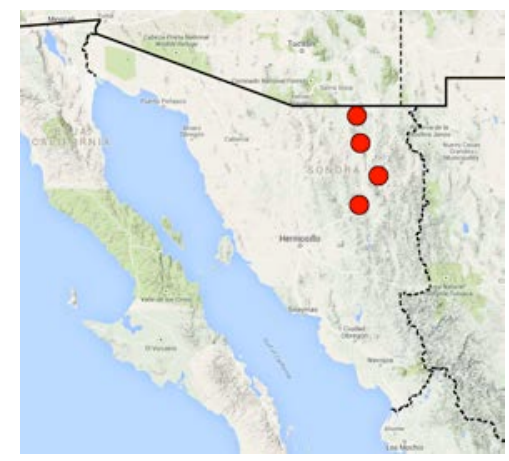

350. Lebia (Lebia) sp. UASM-37A

Number examined: 1.

Municipality: Naco.

Habitat: ?

Vegetation: desert grassland; oak woodland.

Elevation: $1654 \mathrm{~m}$.

Collection date: 2017.IX.16.

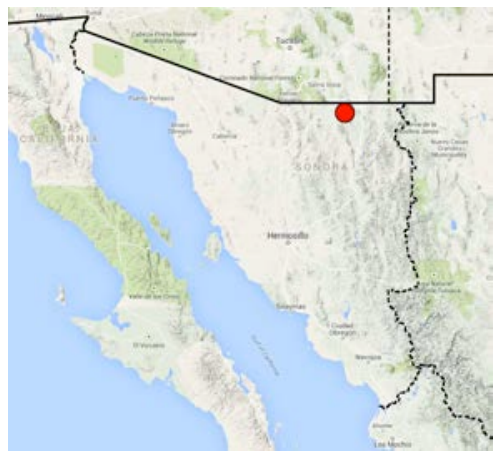

351. Lebia (Lebia) sp. UASM-41

Number examined: 3 .

Municipalities: Agua Prieta, Moctezuma.

Habitat: [at light]

Vegetation: foothills thornscrub.

Elevation range: $1000-1350 \mathrm{~m}$.

Collection dates: 1982: VIII.19-VIII.20; 1993.VII.04.

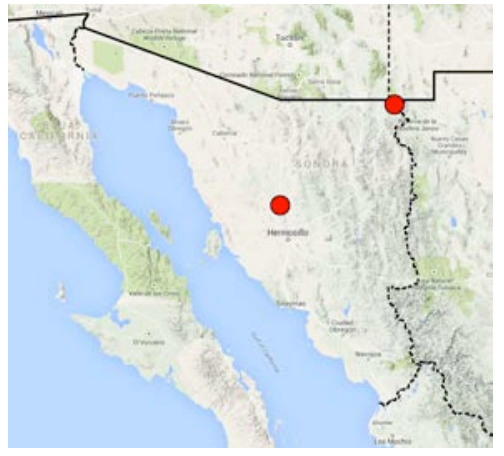

352. Lebia (Lebia) sp. UASM-74

Number examined: 1.

Municipality: Yécora.

Habitat: [at light]

Vegetation: oak woodland.

Elevation: $1680 \mathrm{~m}$.

Collection date: 1993.VII.08.

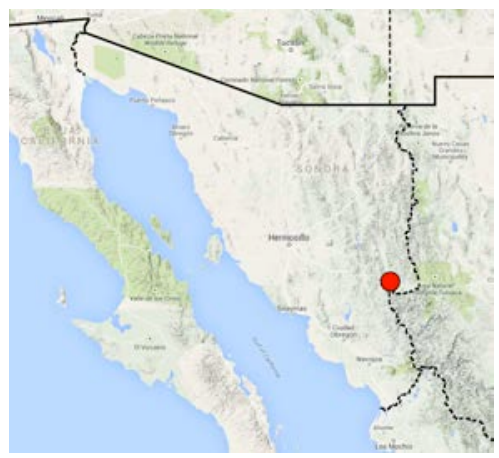

353. Lebia (Lebia) sp. UASM-75

Number examined: 23 .

Municipalities: Huachinera, Nácori Chico.

Habitat: meadow; rocky stream canyon. [at light]

Vegetation: cypress-maple-alder-sycamore riparian forest; oak woodland; pine-oak forest.

Elevation range: $1648-2090 \mathrm{~m}$.

Collection dates: 1982: VIII.03-VIII.04, VIII.06-VIII.07; 2018: VIII.05-VIII.09.

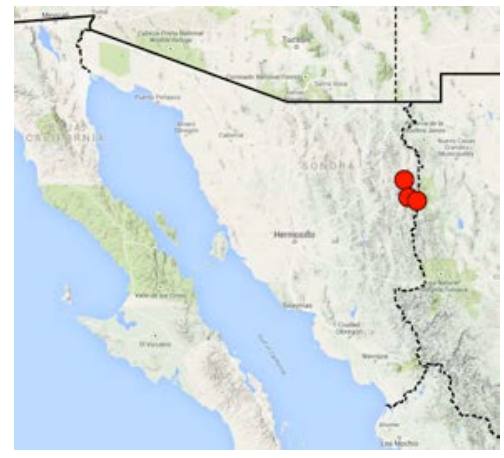


354. Lebia (Lebia) sp. UASM-78

Number examined: 1.

Municipality: Nogales.

Habitat: rocky canyon.

Vegetation: riparian forest, oak woodland.

Elevation: $1200 \mathrm{~m}$.

Collection date: 2012.VII.21.

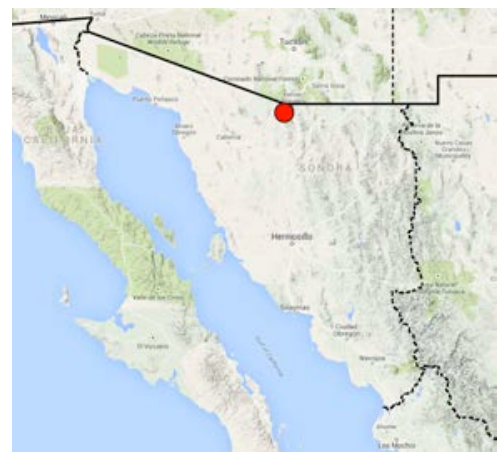

355. Lebia (Lebia) sp. UASM-97

Number examined: 1.

Municipality: Álamos.

Habitat: [at light]

Vegetation:?

Elevation: $1577 \mathrm{~m}$.

Collection date: 1989: VII.11-VII.13.

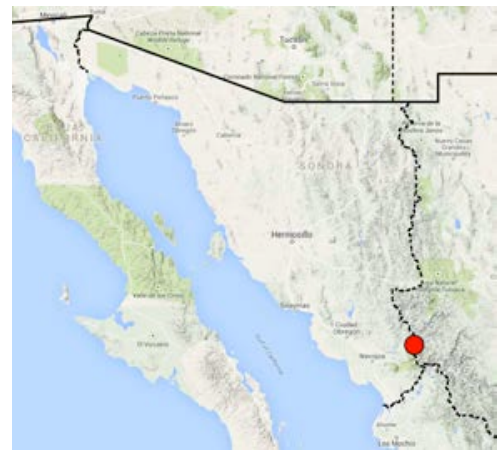

356. Lebia (Lebia) sp. UASM-123

Number examined: 1.

Municipality: Yécora.

Habitat: [at light]

Vegetation: oak woodland; pine-oak forest.

Elevation: $1680 \mathrm{~m}$.

Collection date: 1993.VII.08.

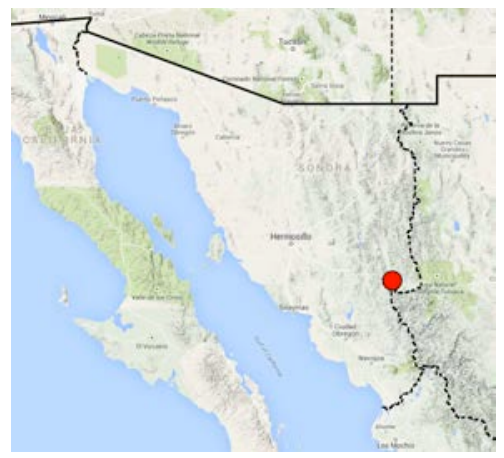

357. Lebia (Lebia) sp. UASM-140

Number examined: 5 .

Municipality: Nácori Chico, Nacozari de García.

Habitat: rocky mountainside. [at light]

Vegetation: oak woodland; Sonoran desertscrub.

Elevation range: $930-1687 \mathrm{~m}$.

Collection dates: 1982.VIII.05; 2017.VII.14.

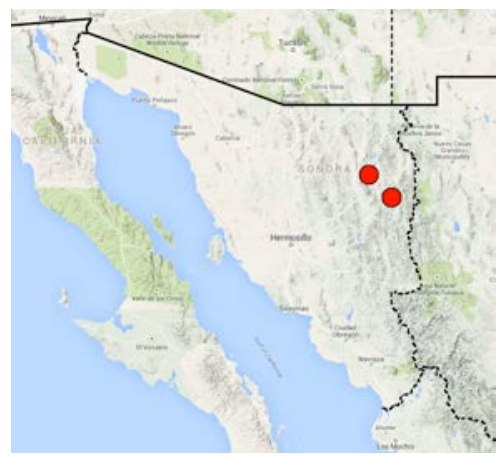

358. Lebia (Lebia) sp. UASM-167

Number examined: 16.

Municipality: Nácori Chico.

Habitat: rocky stream canyon. [at light]

Vegetation: cypress-maple-alder-sycamore riparian forest; pine-oak forest.

Elevation Range: $1648-1715 \mathrm{~m}$.

Collection dates: 2018: VI.27, VIII.05-VIII.06, VIII.09.

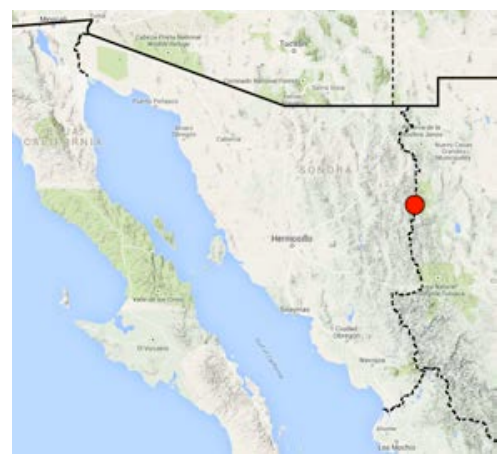

359. Lebia (Loxopeza) atriceps LeConte, 1863

Number examined: 40.

Municipalities: Aconchi, Agua Prieta, Álamos, Bacadehuachi, Bacanora, Cananea, Fronteras, Huachinera, Mag- 
dalena de Kino, Moctezuma, Naco, Nacozari de García, Nogales, Yécora.

Habitat: arroyo; canyon, slope, mountainside; rocky stream; [at light]

Vegetation: cypress-maple-alder-sycamore riparian forest; desert grassland; foothills thornscrub; oak woodland; pine-oak forest; Sonoran desertscrub; tropical deciduous forest.

Elevation range: $944-2422 \mathrm{~m}$.

Collection dates: 1935. VII.07; 1982: VIII.01, VIII.03, VIII.05, VIII.19-VIII.20; 1990: VII.02-VII.03; 1993: VII.08-VII.09; 2012: VII.13, VII.21, IX.02; 2013: VII.16, VIII.02, VIII.09; 2014: VIII.02, VIII.24; 2015. IX.13; 2016.VIII.15; 2017: VII.15, IX.16; 2018.VI.27.

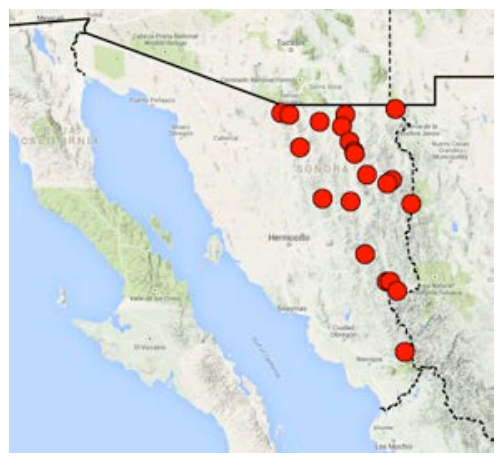

360. Lebia (Loxopeza) deceptrix Madge, 1967

Number examined: 16.

Municipalities: Fronteras, Naco, Nacozari de García, Yécora.

Habitat: arroyo; rocky mountainside. [at light]

Vegetation: oak woodland; sycamore riparian forest.

Elevation range: $1646-1752 \mathrm{~m}$.

Collection dates: 1990: VI.28-VI.29, VII.02-VII.03; 2016.08.15; 2017: VII.15, VII.15, IX.16.

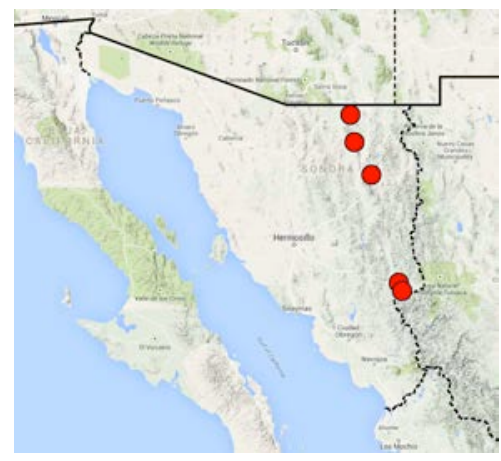

361. Lebia (Loxopeza) pimalis (Casey, 1920)

Number examined: 171.

Municipalities: Aconchi, Álamos, Arizpe, Bacadehuachi, Bacanora, Baviácora, Cananea, Fronteras, Magdalena de Kino, Moctezuma, Naco, Nacozari de García, Nogales, Onavas, Opodepe, San Felipe de Jesús, Santa Ana, Ures, Yécora.

Habitat: arroyo; mountainside, and ridge top; rocky slope, stream canyon, slope, hillside; [at light]

Vegetation: desert grassland; foothills thornscrub; oak woodland; pine-oak forest; Sonoran desertscrub; sycamore riparian deciduous forest; tropical deciduous forest.

Elevation range: $518-2467 \mathrm{~m}$.

Collection dates: 1935.VII.07; 1968.X.01; 1980: IX.24, IX.27; 1982: VIII.01, VIIII.03, VIII.05; 1987: VII.30VII.31; 1989: VII.11-VII.13; 1990: VII.02-VII.03, VIII.06; 1993: VII.08-VII.09, VII.14; 2012: VII.07, VII.13, VII.21, VIII.09, IX.02; 2013: VII.16-VII.17; 2014: VII.08, VIII.02, VIII.24, IX.14; 2015: VIII.08, VIII.12, IX.13; 2016: VIII.14-VIII.15; 2017: I.11, VII.15, VIII.12, VIII.14-VIII.15, IX.16; 2019: VII.06, VIII.05.

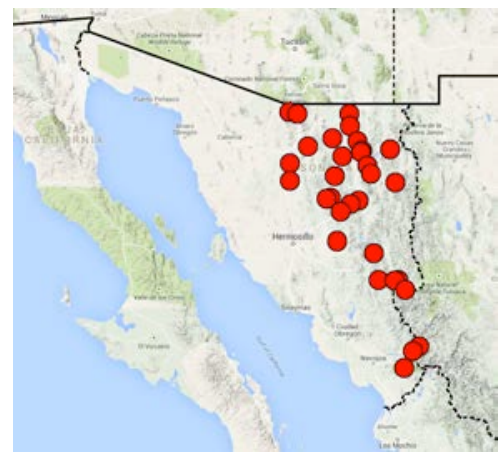

362. Lebia (Loxopeza) subdola Madge, 1967

Number examined: 6 .

Municipalities: Cananea, Fronteras, Nacozari de García.

Habitat: arroyo; rocky stream canyon, slope, and ridge. [at light]

Vegetation: oak woodland; pine-oak forest on slopes; sycamore riparian deciduous forest.

Elevation range: $1698-2467 \mathrm{~m}$.

Collection dates: 2013: VII.17, VIII.02; 2014.VIII.24; 2016.VIII.16.

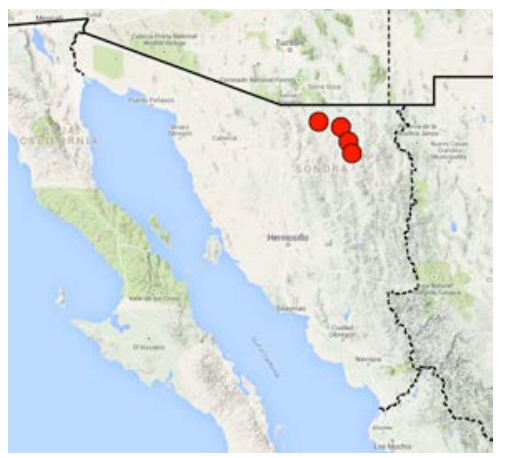

363. Lebia (Loxopeza) subgrandis Madge, 1967

Number examined: 104.

Municipalities: Aconchi, Agua Prieta, Álamos, Arizpe, Baviácora, Cananea, Carbo, Fronteras, Hermosillo, la Colorada, Mazatán, Moctezuma, Naco, Nácori Chico, Nacozari de García, Nogales, Navojoa, Onavas, 
Opodepe, Santa Ana, Ures.

Habitat: cow pasture; rocky stream canyon, slope, hillside, and mountainside. [at light]

Vegetation: Chihuahuan and Sonoran desertscrub; coastal and foothills thornscrub; cypress-maple-alder-sycamore riparian forest; desert grassland; oak woodland; pineoak forest; tropical deciduous forest.

Elevation range: $229-2238 \mathrm{~m}$.

Collection dates: 1954.VIII.22; 1957: VII.28-VII.29; 1960. VIII.08; 1963.VII.06; 1967.VII.27; 1980: IX.18, IX.24; 1982: VIII.01, VIII.05, VIII.19-VIII.20, VIII.23; 1983: VII.04, VII.11-VII-12; 1990.VIII.06; 1993.VII.04; 2012: VII.07, VII.21, VIII.06, VIII.09, VIII.13; 2014: VII.28, VIII.26, IX.14; 2015: VII.12, VIII.08; 2016: VII.02, VIII.14-VIII.16; 2017: I.11, IV.23, VII.15, VIII.16, IX.16; 2018. VIII.06.

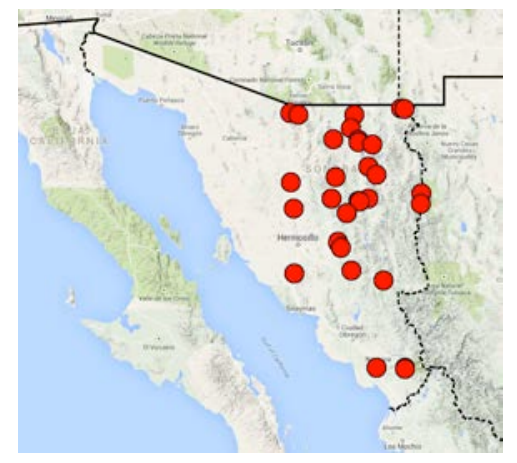

364. Lebia (Loxopeza) sp. son-7

Number examined: 37.

Municipalities: Álamos, Onavas, Yécora.

Habitat: [at light]

Vegetation: foothills thornscrub; oak woodland; tropical deciduous forest.

Elevation range: $518-1577 \mathrm{~m}$.

Collection dates: 1960.VIII.12; 1964.VIII.05; 1965.VII; 1987: VII.26-VII.27, VII.30-VII.31; 1989: VII-11VII.13; 1993.VII.09.

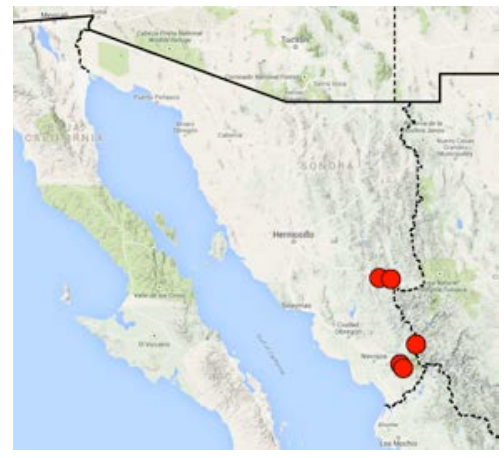

365. Lebia (Loxopeza) sp. son- 8

Number examined: 19.

Municipalities: Álamos, Bacanora, Nacozari de García, Onavas, Ures, Yécora.

Habitat: rocky slope and mountainside; [at light]
Vegetation: foothills thornscrub; oak woodland; pine-oak forest; tropical deciduous forest.

Elevation range: $518-1752 \mathrm{~m}$.

Collection dates: 1960.VIII.12; 1987: VII.26-VII.27, VII.30-VII.31; 1990: VI.30-VII.03, VIII.06; 1993. VII.09; 2014: VII.28, VIII.02; 2017.VII.15.

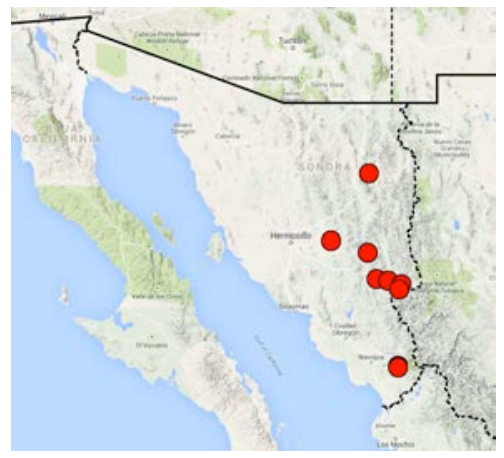

366. Lebia (Loxopeza) sp. UASM-136

Number examined: 7.

Municipalities: Álamos, Yécora.

Habitat: [at light]

Vegetation: oak woodland; pine-oak forest; tropical deciduous forest.

Elevation range: $1577-1680 \mathrm{~m}$.

Collection dates: 1989: VII.11-VII.13; 1993.VII.08.

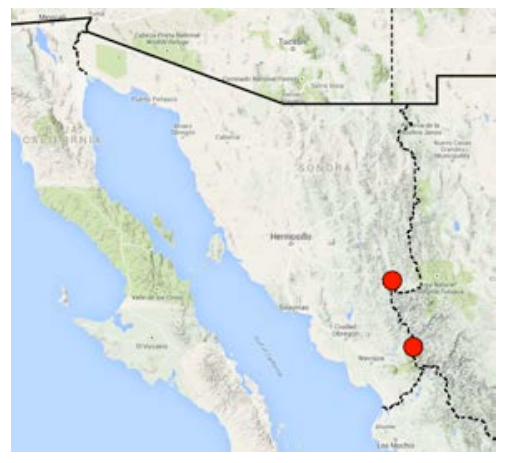

367. Lebia (Polycheloma) sp. UASM-31 (= sp. CASC 104)

Number examined: 25

Municipalities: Álamos, Hermosillo, Ímuris, Onavas.

Habitat: in bromeliads. [at light]

Vegetation: ciénega in cottonwood-willow riparian deciduous forest; foothills thornscrub; Sonoran desertscrub; tropical deciduous forest

Elevation range: $229-917 \mathrm{~m}$.

Collection dates: 1935.VIII.18; 1960.VIII.12; 1964. VIII.08. 1967.VII.27; 1987: VII.30-VII.31; 1990. VIII.06. 


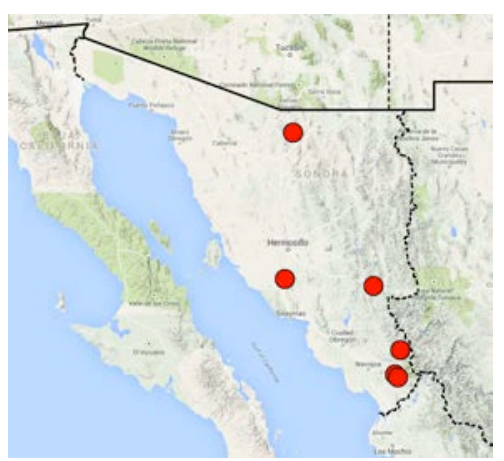

368. Lebia (Polycheloma) sp. UASM-164

Number examined: 15.

Municipality: Agua Prieta.

Habitat: [at light]

Vegetation: cottonwood-willow riparian deciduous forest.

Elevation: $1350 \mathrm{~m}$.

Collection dates: 1982: VIII.19-VIII.20.

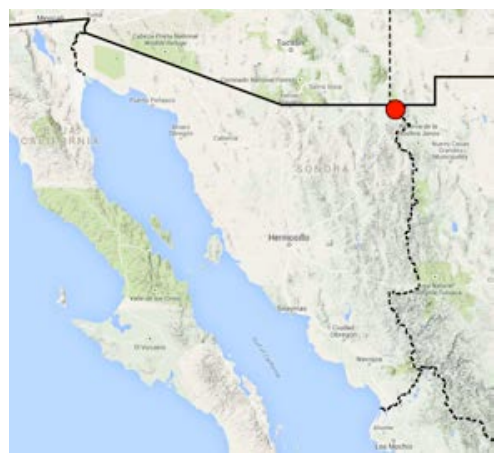

\section{Nemotarsus sp.}

Number examined: 1.

Municipality: Ónavas.

Habitat: [at light]

Vegetation: foothills thornscrub.

Elevation: $917 \mathrm{~m}$.

Collection date: 1987: VII.26-VII.27.

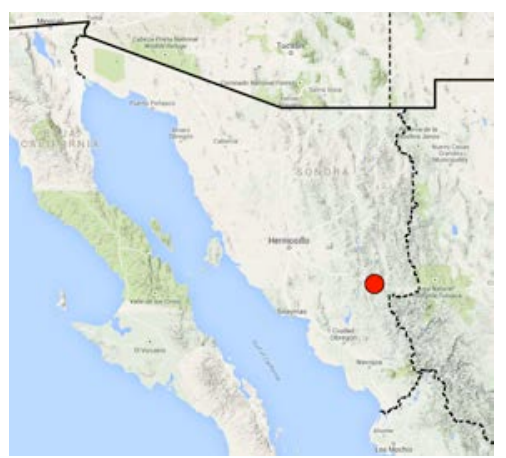

370. Philophuga caerulea Casey, 1913

Number examined: 9 .

Municipalities: Cananea, Naco, Nacozari de García.

Habitat: rocky canyon, slope, and ridge.
Vegetation: desert grassland; oak woodland; pine-oak forest.

Elevation range: $1654-2467 \mathrm{~m}$.

Collection dates: 2013: VIII.02, IX.08-IX.09, IX.11; 2014.

IX.08; 2017.IX.16.

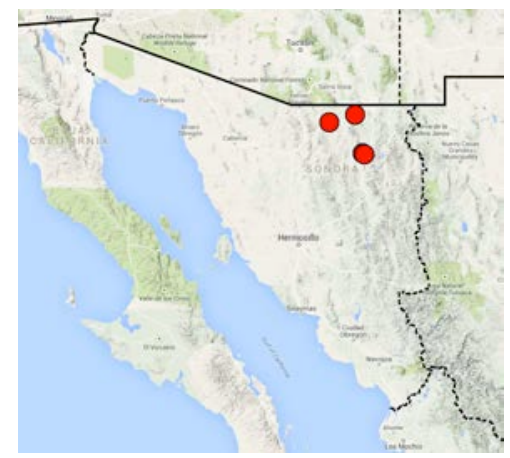

371. Philophuga viridis viridis (Dejean, 1831)

Number examined: 1.

Municipality: Nogales.

Habitat: ?

Vegetation: desert grassland.

Elevation: ?

Collection date: 1965.IX.28.

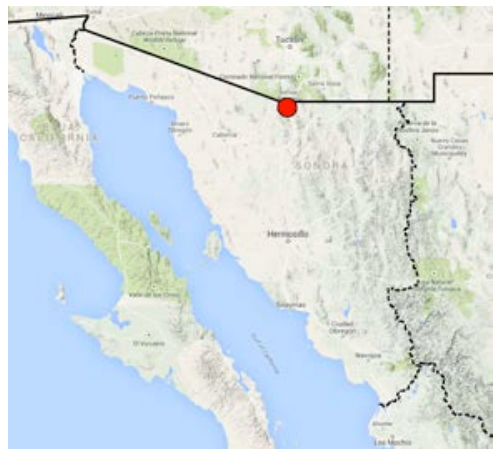

372. Plochionus timidus Haldeman, 1843

Number examined: 5 .

Municipalities: Álamos, Bacadéhuachi, Pitiquito.

Habitat: rocky canyon and mountainside.

Vegetation: sycamore-Cupressus arizonica riparian forest, pine-oak forest on slopes; tropical deciduous forest.

Elevation range: $200-1680 \mathrm{~m}$.

Collection dates: 1935: VII.07, VII.15; 1953: VIII.08VIII.10; 1976.IX.28; 2012.III.30. 


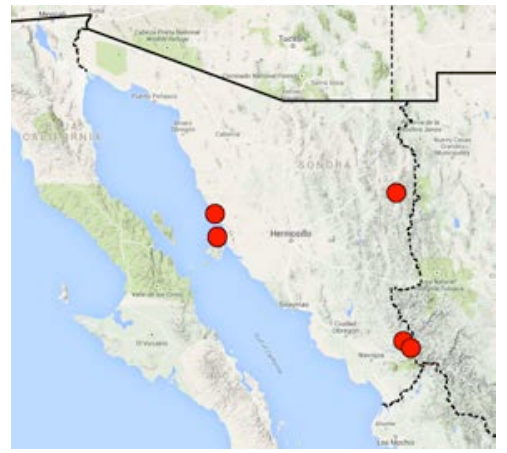

\section{Zuphiini}

373. Thalpius simplex (Liebke, 1934)

Number examined: 10.

Municipalities: Álamos, Guaymas, Ónavas, Santa Ana.

Habitat: [at light]

Vegetation: foothills thornscrub; tropical deciduous forest. Elevation: $917 \mathrm{~m}$.

Collection dates: 1964.VIII.24; 1973.V; 1987: VII.26VII.27; 1989.VII.08; 2018.III.19.

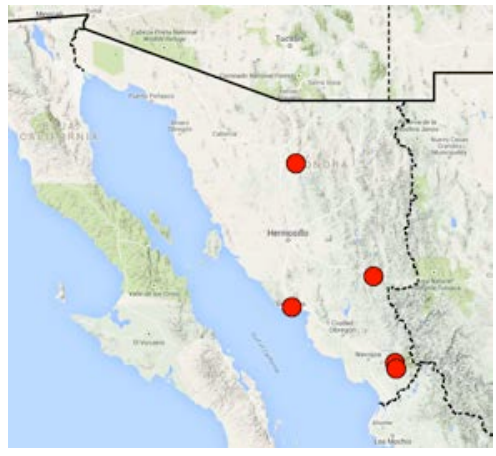

374. Thalpius sp. CASC 9

Number examined: 1 .

Municipality: Hermosillo.

Habitat: ?

Vegetation: Sonoran desertscrub.

Elevation:?

Collection date: 1953: VII.09-VII.16.

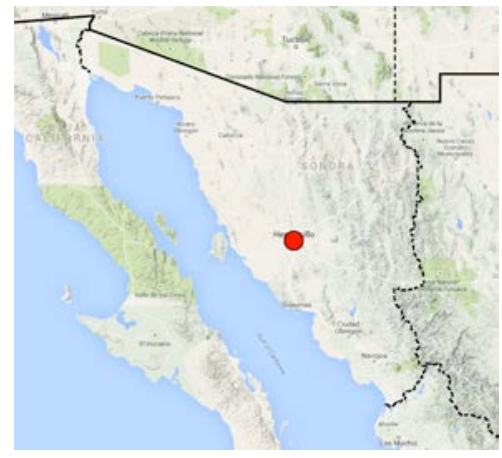

33.Galeritini

375. Galerita atripes LeConte, 1858 Number examined: 16.
Municipalities: Álamos, Fronteras, Nácori Chico, Nacozari de García, Yécora.

Habitat: rocky arroyo and mountainside. [at light]

Vegetation: Chihuahuan desertscrub; oak woodland; oak-juniper-sycamore riparian forest; pine-oak forest; tropical deciduous forest.

Elevation range: 1577 - $1950 \mathrm{~m}$.

Collection dates: 1983.VII.06; 1989: VII.11-VII.113; 1990: VI.28-VI.29, VIII.07-VIII.11; 2016: VIII.14, VIII.16; 2017.VII.15.

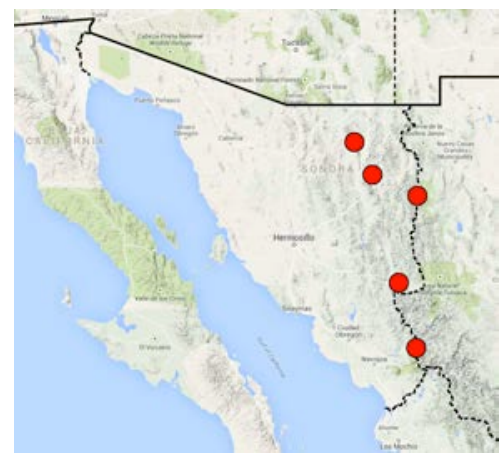

376. Galerita forreri Bates, 1883

Number examined: 19.

Municipalities: Aconchi, Álamos, Bacanora, Cananea, Fronteras, Moctezuma, Nacozari de García, Nogales, Ónavas, San Felipe de Jesús.

Habitat: rocky arroyo, canyon, hillside, and mountainside. Vegetation: Chihuahuan desertscrub; desert grassland; foothills thornscrub; oak woodland; oak-juniper-sycamore riparian forest.

Elevation range: $433-1708 \mathrm{~m}$

Collection dates: 1983.VII.06; 1987: VII.26-VII.27; 1993.VII.05; 2012: VII.21, IX.02; 2013.VII.02; 2014: VIII.02, IX.14; 2016.VIII.15; 2017.VIII.14; 2019: VII.24, VIII.06.

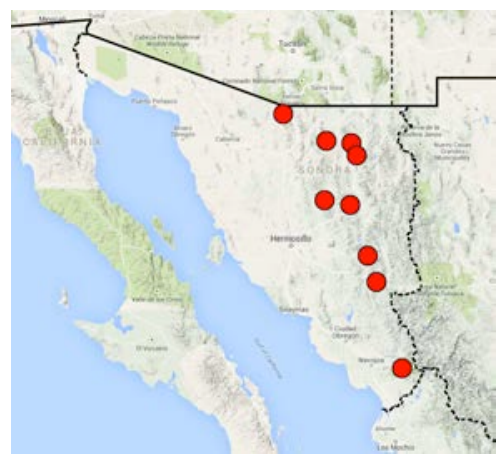

377. Galerita lecontei Dejean, 1831

Number examined: 24.

Municipalities: Agua Prieta, Ímuris, Nácori Chico, Santa Ana.

Habitat: stream margin.

Vegetation: riparian forest.

Elevation range: $1040-1450 \mathrm{~m}$. 
Collection dates: 1982: VIII.20-VIII.21, VIII.24; 1983. VII.05; 1985.VIII.02.

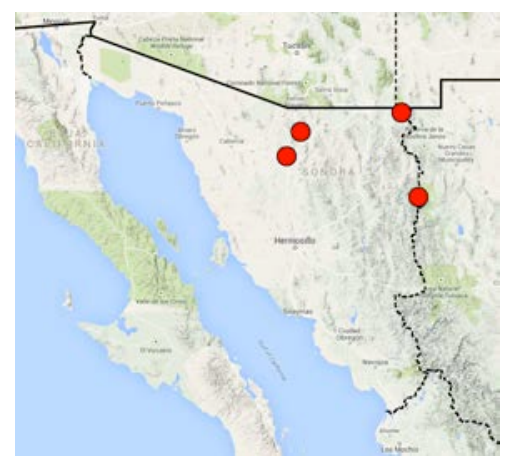

378. Galerita mexicana Chaudoir, 1872

Number examined: 62 .

Municipalities: Agua Prieta, Álamos, Fronteras, Hermosillo, Moctezuma, Nogales, Ónavas, Rosario, Santa Ana, Santa Cruz.

Habitat: cow pasture; lights at building at night. [at light] Vegetation: Chihuahuan and Sonora desertscrub; desert grassland; foothills thornscrub; oak woodland; tropical deciduous forest.

Elevation range: $376-1429 \mathrm{~m}$.

Collection dates: 1953: VII.09-VII.16; 1958.IV.03; 1980: VII.21-VII.22; 1982: VIII.01, VIII.19-VIII.20; 1983: VII.11-VII.12; 1984.VII.21; 2004.X.18; 1987: VII.26VII.27; 1993.VII.04; 2012.IX.12; 2013: VIII.09, IX.11; 2015.VII.12; 2016.VII.02; 2017.VII.23; 2018.VIII.13.

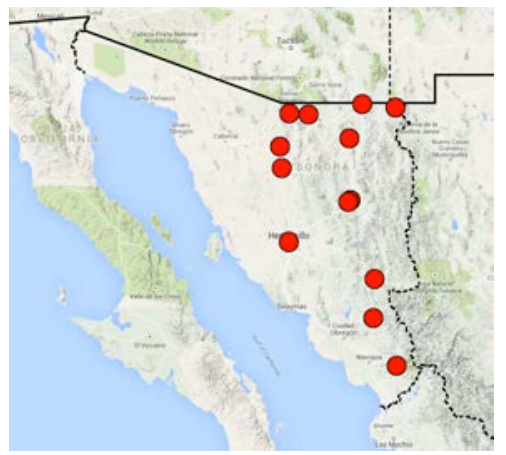

379. Galerita ruficollis Dejean, 1825

Number examined: 17.

Municipalities: Álamos, Carbó, Guaymas, Navojoa, Rosario.

Habitat: [at light]

Vegetation: coastal and foothills thornscrub; Sonoran desertscrub; tropical deciduous forest.

Elevation range: $46-518 \mathrm{~m}$.

Collection dates: 1960: VIII.08, VIII.12; 1962.VII.12; 1984: 07.02, VII.21; 1987: 07.30-07.31; 1989.07.08; 1992: X.03-X.16; 2012.09.12.

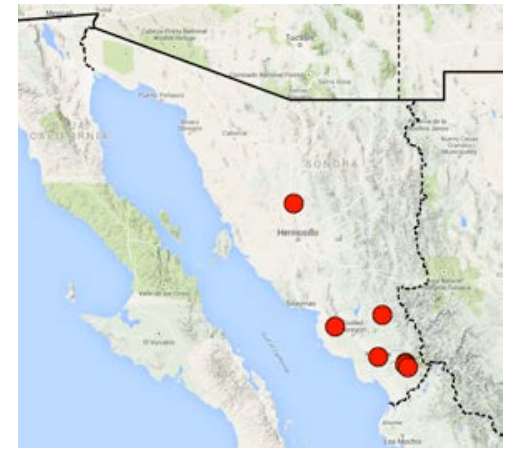

\section{Helluonini}

380. Helluomorphoides ferrugineus LeConte, 1853

Number examined: 7.

Municipalities: Álamos, Cananea, Hermosillo, Moctezuma, Naco, Nacozari de García.

Habitat: rocky hillside.

Vegetation: desert grassland; oak woodland; Sonoran desertscrub; tropical deciduous forest.

Elevation range: $268-1654 \mathrm{~m}$.

Collection dates: 1962.VII.13; 1982.VIII.11; 1983: VII.11-VII.12; 2012.IX.13; 2015: IX.06; 2017.IX.16.

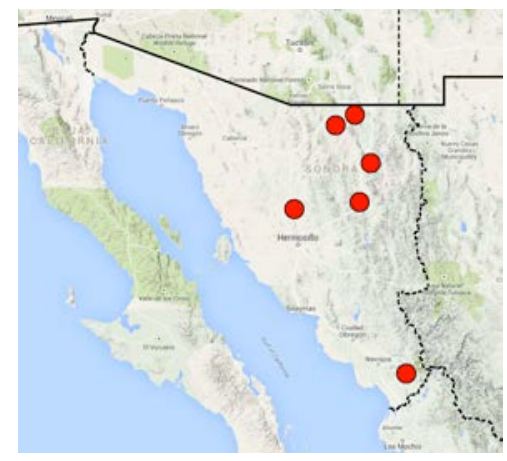

381. Helluomorphoides latitarsis (Casey, 1913)

Number examined: 23.

Municipalities: Aconchi, Álamos, Arizpe, Fronteras, Mazatán, Moctezuma, Nacozari de Garcia, Nogales, San Felipe de Jesús.

Habitat: cow pasture; rocky canyon, slopes, and mountainside; slopes.

Vegetation: desert grassland; foothills thornscrub; oak woodland; sycamore riparian deciduous forest; tropical deciduous forest.

Elevation range: $944-1707 \mathrm{~m}$.

Collection dates: 1935.VI.26; 1957: VII.28-VII.29; 1982: VIII.01, VIII.11; 1983: VII.11-VII.12; 1993.VII.04; 2012: VII.21, VIII.01; 2013.VII.02; 2014.VII.28; 2015: VIII.08; 2016: VII.02, VIII.14; 2019: VII.06, VIII.04. 


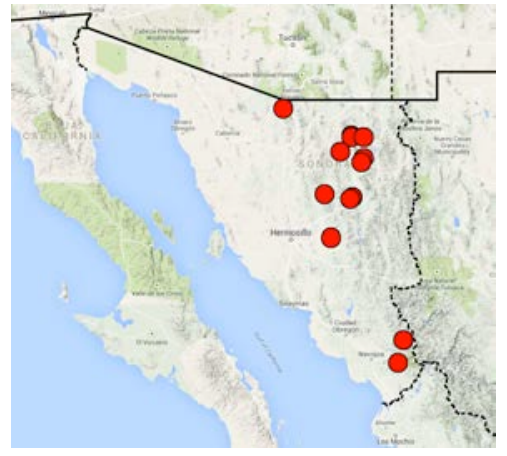

382. Helluomorphoides sp. CASC 2

Number examined: 1.

Municipality: Álamos.

Habitat: ?

Vegetation: tropical deciduous forest.

Elevation: ?

Collection date: 1935.VII.20.

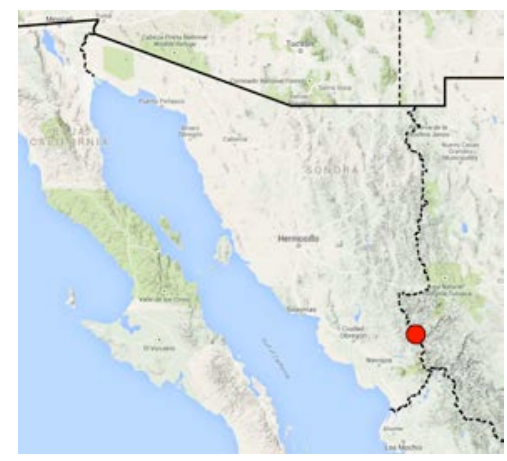

35.Pseudomorphini

383. Pseudomorpha huachinera Erwin \& Amundson, 2013

Number examined: 1.

Municipality: Yécora.

Habitat: ?

Vegetation: pine-oak forest.

Elevation: ?

Collection date: 1987: VIII.28-VIII.29.

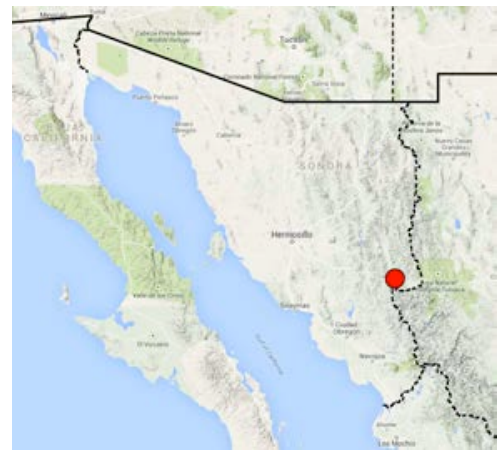

384. Pseudomorpha angustata group sp. \#1

Number examined: 1

Municipality: Santa Ana.
Habitat: Sonoran desertcrub.

Vegetation:?

Elevation:?

Collection date: 1982.VIII.23.

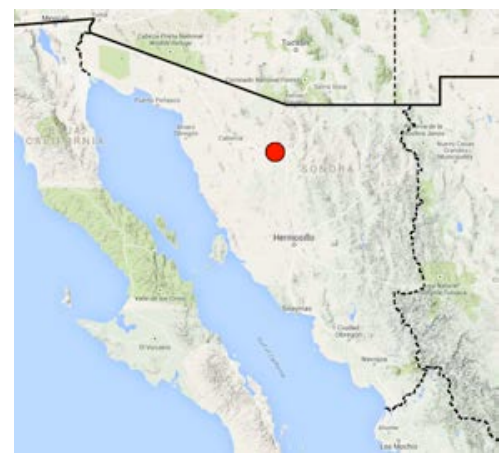

385. Pseudomorpha angustata group sp. \#2

Number examined: 2.

Municipality: Nacozari de García.

Habitat: rocky canyon.

Vegetation: oak woodland.

Elevation: $1595 \mathrm{~m}$.

Collection date: 2013.VII.16.

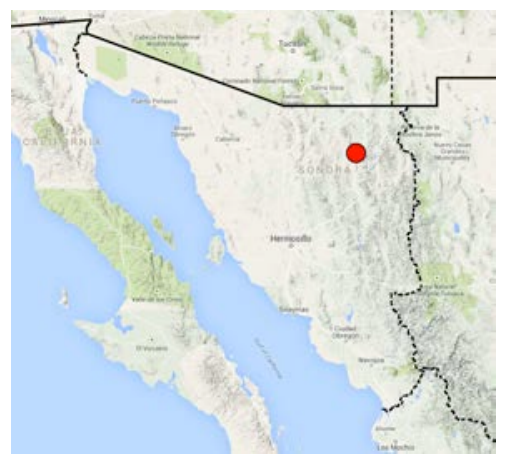

386. Pseudomorpha angustata group sp. \#3

Number examined: 1

Municipality: Nacozari de García.

Habitat: rocky mountainside.

Vegetation: oak woodland

Elevation: $1687 \mathrm{~m}$.

Collection date: 2017.VIII.15.

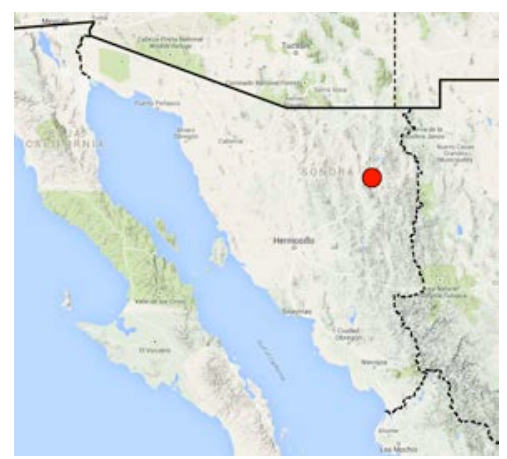

387. Pseudomorpha santarita group sp. 
Number examined: 1

Municipality: Fronteras.

Habitat: arroyo. [at light]

Vegetation: oak-sycamore-juniper forest.

Elevation: $1730 \mathrm{~m}$.

Collection date: 2016.VIII.16.

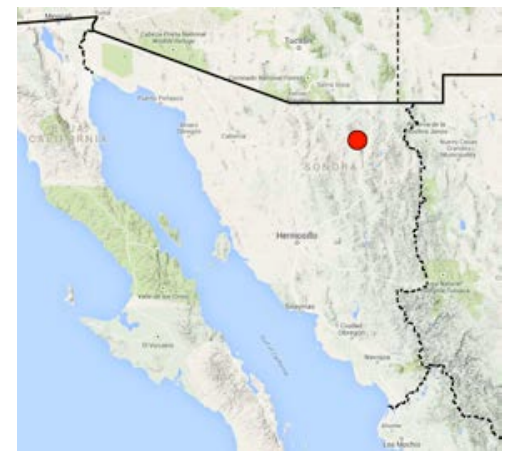

388. Pseudomorpha tenebriodes group sp.

\section{Number examined: 1}

Municipio: Santa Ana.

Habitat: Sonoran desert.

Vegetation:?

Elevation:?

Collection date: 1982.VIII.23.

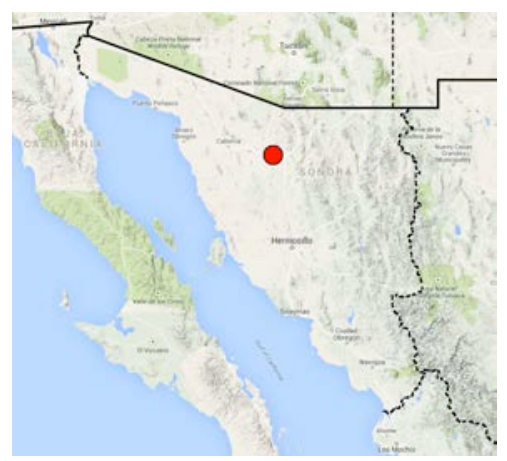

\section{LITERATURE CITED}

Ball, G.E. and D.R. Maddison. 1987. Classification and evolutionary aspects of the species of the New World genus Amblygnathus Dejean, with description of Platymetopsis, new genus, and notes about selected species of Selenophorus Dejean (Coleoptera: Carabidae: Harpalini). Transactions of the American Entomological Society 113: 189-307.

Ball, G.E. and J. Nègre. 1972. The taxonomy of the Nearctic species of the genus Calathus Bonelli (Coleoptera: Carabidae: Agonini). Transactions of the American Entomological Society 98: 413-533.

Ball, G.E. and D. Shpeley. 2000. Chapter 19, Carabidae (Coleoptera). (pp. 363-399). In: Bousquets, J.L., E.G. Soriano and N. Papavero (Eds.). Biodiversidad, taxonomía y biogeographía de arthrópodos de México: Hacia una sintesis du su conocimiento Volumen II. Universidad Nacional Autónoma de Mexico, Mexico
D.F. 676 pp.

Ball, G.E. and D. Shpeley. 2002. The Neotropical subgenera and species of the Pantropical Genus Anaulacus MacLeay (sensu novo) (Coleoptera: Carabidae: Masoreini): a taxonomic revision, with notes about way of life, evolution, and geographical history. Transactions of the American Entomological Society 128:(2+3): 265343.

Bousquet, Y. 2012. Catalogue of Geadephaga (Coleoptera, Adephaga) of America, north of Mexico. ZooKeys 245: $1-1722$.

Cazier, M.A. 1954. A review of the Mexican tiger beetles of the genus Cicindela (Coleoptera, Cicindelidae). Bulletin of the American Museum of Natural History 103: 231-309.

Deyo, N.S., A. Smith, E. Gilbert and T.R. Van Devender. 2013. Documenting the biodiversity of the Madrean Archipelago: an analysis of a virtual flora and fauna. (pp. 292-299). In: Gottfried, G.J., P.F. Ffolliott, B.S. Gebow, L.G. Eskew, and L.C. Collins (compilers). Merging science and management in a rapidly changing world: biodiversity and management of the Madrean Archipelago III and 7th Conference on Research and Resource Management in the Southwestern Deserts. 2012 May 1-5, Tucson, AZ. Proceedings RMRS-P-67. Fort Collins, CO: U.S. Department of Agriculture, Forest Service, Rocky Mountain Research Station. 593 pp.

Erwin, T.L. 1970. A reclassification of bombardier beetles and a taxonomic revision of the North and Middle American species (Carabidae: Brachinida). Quaestiones Entomologicae 6: 4-215.

Gentry, H.S. 1942. Rio Mayo Plants. Carnegie Institute of Washington Publication 527, Washington, D. C. 328 pp.

Gidaspow, T. 1959. North American caterpillar hunters of the Genera Calosoma and Callisthenes (Coleoptera, Carabidae). Bulletin of the American Museum of Natural History 116: 225-343.

González-E., M.S., M. González-E., J.A. Tena-Flores, L. Ruacho-G. and I.L. López-E. 2012. Vegetación de la Sierra Madre Occidental. Una síntesis. Acta Botanica Mexicana 100: 351-403.

Instituto Nacional de Estadística y Geografía (INEGI). Marco Geoestadístico (Sonora map with municipalities coded with numbers, and list of names corresponding to each municipality number). Retrieved: April 11, 2016. http://www.cuentame.inegi.org.mx/mapas/pdf/ entidades/div_municipal/sonorampios.pdf

Martin, P.S., D.A. Yetman, M. Fishbein, P. Jenkins, T.R. Van Devender and R.K. Wilson (eds.). 1998. Gentry's Rio Mayo Plants. The Tropical Deciduous Forest and Environs of Northwest Mexico. Univ. of Arizona Press, Tucson. 558 pp. 
Messer, P.W. and B.Y. Raber, 2021. A review of Nearctic Selenophorus Dejean (Coleoptera: Carabidae: Harpalini) north of Mexico with new species, new synonyms, range extensions, and a key. The Coleopterists Bulletin 75(1): 9-55.Noonan, G.R. 1973. The Anisodactylines (Insecta: Coleoptera: Carabida: Harpalini): Classification, Evolution, and Zoogeography. Quaestiones Entomologicae 9: 266-480.

Shpeley, D. 1986. Genera of the Subtribe Metallicina and classification, reconstructed phylogeny and geographical history of the species of Euproctinus Leng \& Mutchler (Coleoptera: Carabidae: Lebiini). Quaestiones Entomologicae 22: 261-349.

Shpeley, D. and G.E. Ball. 1993. Classification, reconstructed phylogeny and geographical history of the New World species of Coptodera Dejean (Coleoptera: Carabidae: Lebiini). Proceedings of the Entomological Society of Ontario 124: 1-182.

Turner, R.M. 1994. Tropical-subtropical desertlands. 154.1 Sonoran desertscrub. (pp. 181-221). In: D.E. Brown (ed.) Biotic Communities Southwestern United States and Northwestern Mexico. University of Utah Press, Salt Lake. 346 pp.

Van Devender, T.R. and A.L. Reina-G. 2016. The tropical Madrean flora of Yécora, Sonora, Mexico. Phytoneuron 7: 1-23.

Van Devender, T.R., G. Yanes-A., A.L. Reina-G., M. Valenzuela-Y., M.P. Montañez-A. and H. Silva-K.

Recibido: 12 de abril 2021

Aceptado: 9 de septiembre 2021 2013a. Comparison of the tropical floras of the Sierra la Madera and the Sierra Madre Occidental, Sonora, Mexico. (pp. 240-242). In: Gottfried, G.J., P.F. Ffolliott, B.S. Gebow, L.G. Eskew, and L.C. Collins (compilers). Merging science and management in a rapidly changing world: biodiversity and management of the Madrean Archipelago III and 7th Conference on Research and Resource Management in the Southwestern Deserts. 2012 May 1-5, Tucson, AZ. Proceedings RMRS-P-67. Fort Collins, CO: U.S. Department of Agriculture, Forest Service, Rocky Mountain Research Station. 593 pp.

Van Devender, T.R., R.S. Felger, M. Fishbein, F. Molina-Freaner, J.J. Sánchez-Escalante y A.L. Reina-G. 2010. Biodiversidad de las plantas vasculares. (pp. 229-262). In: Molina-Freaner, F. y T. R. Van Devender (eds.). Diversidad Biológica de Sonora, Universidad Nacional Autónoma de México, Hermosillo. 496 pp.

Van Devender, T.R., S. Avila-Villegas, M. Emerson, D. Turner, A.D. Flesch and N.S. Deyo. 2013b. Biodiversity in the Madrean Archipelago of Sonora, Mexico. (pp. 10-16). In: Gottfried, G.J., P.F. Ffolliott, B.S. Gebow, L.G. Eskew, and L.C. Collins (compilers). Merging science and management in a rapidly changing world: biodiversity and management of the Madrean Archipelago III and 7th Conference on Research and Resource Management in the Southwestern Deserts. 2012 May 1-5, Tucson, AZ. Proceedings RMRS-P-67. Fort Collins, CO: U.S. Department of Agriculture, Forest Service, Rocky Mountain Research Station. 593 pp.

Wiesner, J. 2020. Checklist of the Tiger Beetles of the World. 2nd Edition. Edition Winterwork, Borsdorf. 540 pp.

Whitehead, D.R. 1972. Classification, phylogeny, and zoogeography of Schizogenius Putzeys (Coleoptera: Carabidae: Scaritini). Quaestiones Entomologicae 8: 131348. 


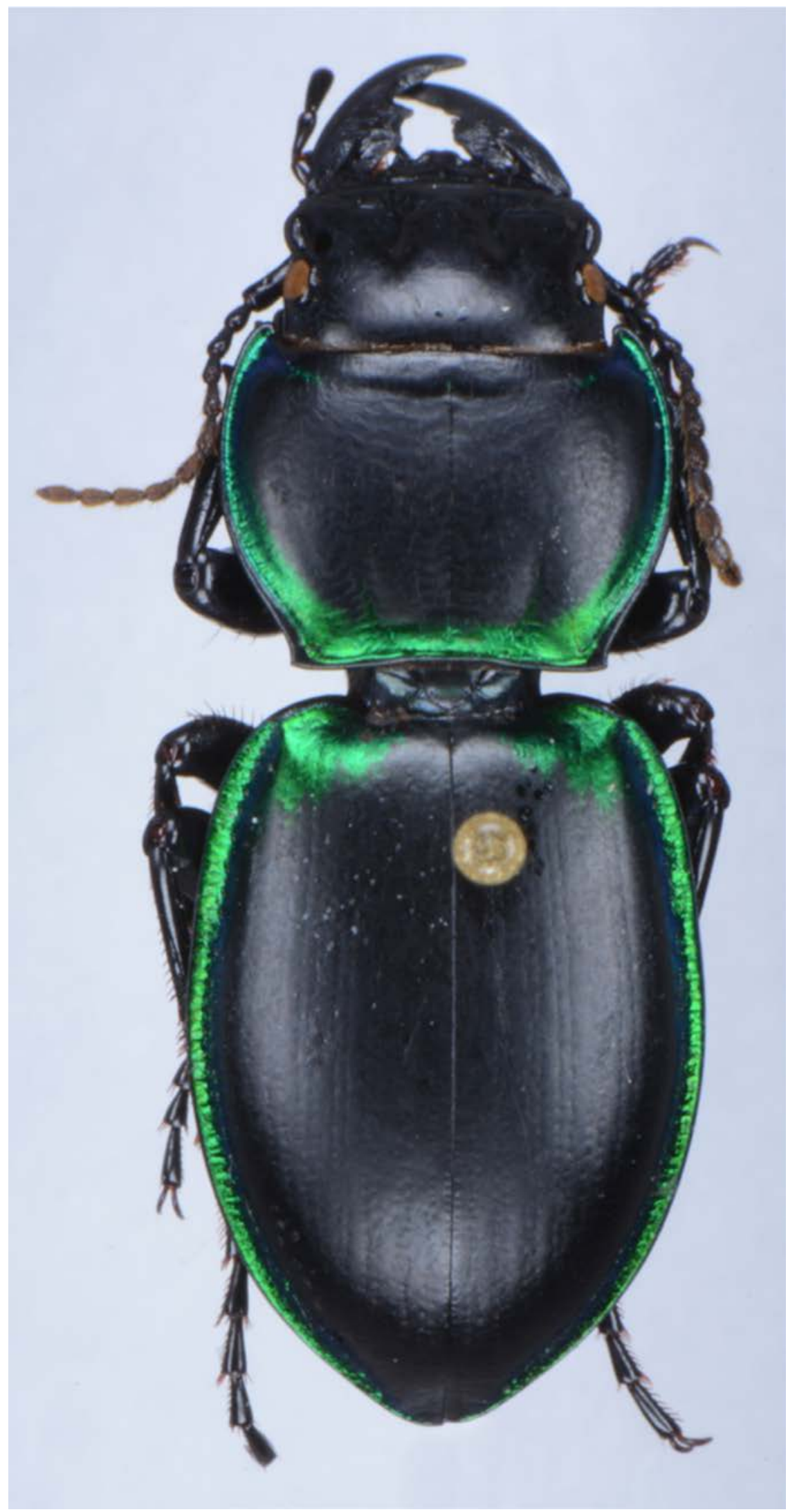

Digital image of Pasimachus viridans LeConte. Locality label data: Mexico, Sonora, Municipality of Nácori Chico, 2 km N Mesa Tres Rios, pine-oak forest, 1950 m, July 6, 1983, H.E. Frania, R. Jaagumagi \& D. Shpeley collectors (overall body length $=27 \mathrm{~mm}$ ). 